\title{
Catalyst-Free and Solvent-Controlled Divergent Synthesis of Difluoromethylene-Containing $S$-Heterocycles
}

\begin{abstract}
Jun-Xiong He, ${ }^{\dagger}$ Zhi-Hao Zhang, ${ }^{\dagger}$ Bo-Shuai Mu,${ }^{\dagger}$ Xiao-Yuan Cui, ${ }^{\dagger}$ Jian Zhou, ${ }^{\dagger, \S}$ and Jin-Sheng Yu, ${ }^{*},, \#$
$\dagger$ Shanghai Engineering Research Center of Molecular Therapeutics and New Drug Development, and Shanghai Key Laboratory of Green Chemistry and Chemical Processes, School of Chemistry and Molecular Engineering, East China Normal University, Shanghai 200062, China.E-mail: jsyu@chem.ecnu.edu.cn

$\S$ State Key Laboratory of Organometallic Chemistry, Shanghai Institute of Organic Chemistry, CAS, Shanghai 200032, China.

\# Key Laboratory of Tropical Medicinal Resource Chemistry of Ministry of Education, Hainan Normal University, Haikou 571158, P R China.
\end{abstract}

\begin{tabular}{|l|c|}
\hline \multicolumn{1}{|c|}{ Table of Contents } & Page \\
\hline 1. The structure and serial number of difluoroenoxysilanes $\mathbf{1}$ and products $\mathbf{4}$ & S2 \\
\hline 2. The possible pathway for the formation of product $\mathbf{5}$ & S3 \\
\hline 3. X-Ray crystal data of $\mathbf{4 h}, \mathbf{5 a}$, and $\mathbf{5 i}$ & S4-S28 \\
\hline $4 .{ }^{1} \mathrm{H},{ }^{13} \mathrm{C}$, and ${ }^{19} \mathrm{~F}$ NMR spectra & S29-S198 \\
\hline
\end{tabular}




\section{The structure and serial number of difluoroenoxysilanes 1 and products 4.}

The structure and serial number of difluoroenoxysilanes $\mathbf{1}$ and C2-adducts $\mathbf{4}$ were shown below for clear.

Table S1. The structure and serial number of difluoroenoxysilanes $\mathbf{1 .}$<smiles>COC(=C(F)F)c1ccc(F)cc1</smiles>

$1 \mathrm{~b}$<smiles>COC(=C(F)F)c1cccc(C)c1</smiles>

$1 f$<smiles>COC(=O)C(F)=C(F)F</smiles>

1c<smiles>COC(=C(F)F)c1ccc(C)o1</smiles>

$1 \mathrm{~g}$<smiles>COC(=C(F)F)c1ccc(C(F)(F)F)cc1</smiles>

1j<smiles>COC(=C(F)F)c1cccs1</smiles>

$1 \mathrm{~h}$<smiles>COc1ccc(C(O[Na])=C(F)F)cc1</smiles>

1d<smiles>COC(=C(F)F)c1ccc2ccccc2c1</smiles>

$1 \mathrm{k}$<smiles>COC(=C(F)F)c1ccc(C)cc1</smiles>

1e<smiles>COC(CCc1ccccc1)=C(F)F</smiles>

$1 \mathrm{i}$

Table S2. The structure and serial number of C2-adducts 4 .

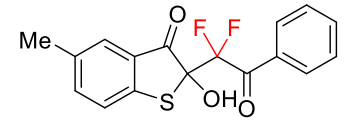

$4 \mathrm{~b}$
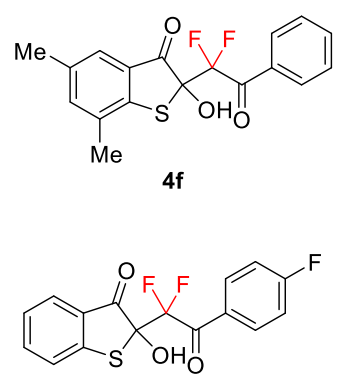

4j

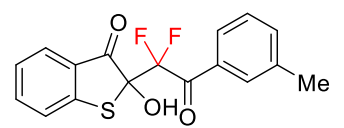

$4 n$

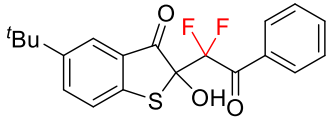

$4 c$

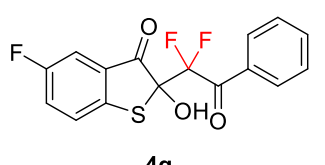

$4 \mathrm{~g}$

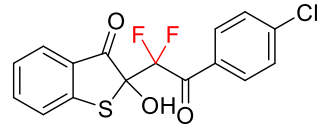

$4 k$

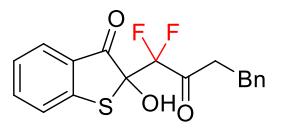

$4 q$

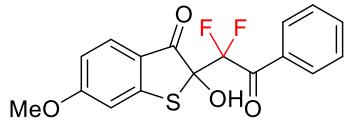

$4 d$

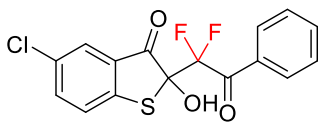

4h

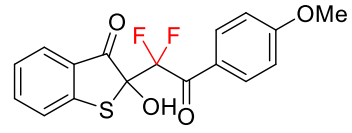

41

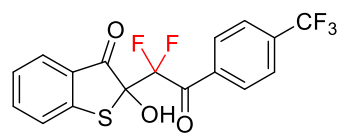

$4 r$
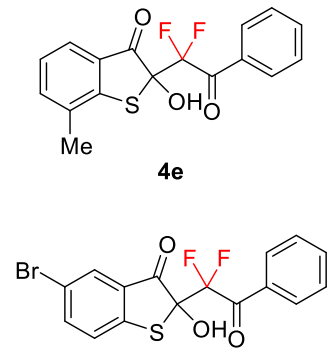

$4 i$

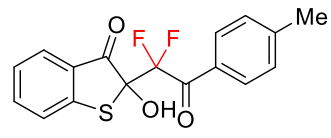

$4 \mathrm{~m}$

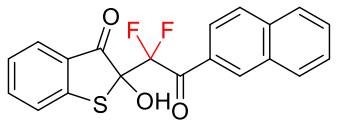

$4 s$ 


\section{The possible pathway for the formation of product 5}

According to the observation of experimental result, the reaction of benzo[b]thiophene-2,3-diones 1a and difluoroenoxysilane 2a in $\mathrm{H}_{2} \mathrm{O}$ delivered the 3-difluoroalkyl substituted 3-hydroxylbenzothiophen2-one 3a firstly, which was subsequently converted into the bridged seven-membered $S$-heterocycle $\mathbf{5 a}$ through the following possible pathway.

The thioester moiety of 3a was attacked by solvent $\mathrm{H}_{2} \mathrm{O}$ and proceeded a ring-opening reaction to deliver an intermediate IM1. The thiophenol anion of IM1 then reacted with the ketone moiety, because the presence of difluoromethyl makes the ketone group more reactive. Subsequently, the resultant intermediate IM2 went through a cyclization to provide the bridged seven-membered $S$-heterocycle $\mathbf{5 a}$. The experimental result of $\mathbf{3 a}$ and $\mathrm{CH}_{3} \mathrm{NH}_{2}$ in ethanol also supports the possibility of this hypothesis (Scheme 2B in the main text).

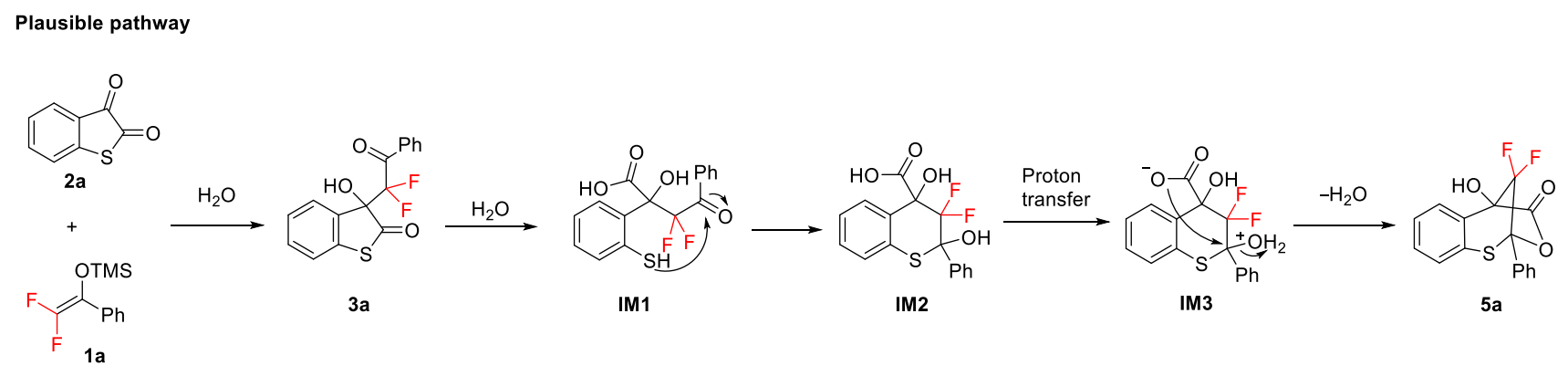




\section{X-Ray crystal data of $4 \mathrm{~h}, 5 \mathrm{a}$, and $5 \mathrm{i}$}

Single crystals of $\mathbf{4 h}, \mathbf{5 a}$, and $\mathbf{5 i}$ were obtained by slow diffusion of its solution in $n$-hexane/EtOAc at room temperature in air.

Data intensity of $\mathbf{4} \mathbf{h}^{1}$ was collected using a 'XtaLAB AFC12 (RINC) ' diffractometer at 292.69(11) K. Data collection and reduction were done by using Olex2 and the structure was solved with the ShelXS structure solution program using direct methods and refined by full-matrix least-squares on $F^{2}$ with anisotropic displacement parameters for non-H atoms using SHELX-97. Hydrogen atoms were added at their geometrically idea positions and refined isotropically. Crystal data for $\mathbf{4 h}: \mathrm{C}_{16} \mathrm{H}_{9} \mathrm{ClF}_{2} \mathrm{O}_{3} \mathrm{~S}$, $T=292.69(11) \mathrm{K}$, monoclinic, $\mathrm{P} 2{ }_{1} / \mathrm{c}, a=15.5043(10) \AA, b=15.5994(10) \AA, c=6.4142(4) \AA, \alpha=90^{\circ}$, $\beta=96.411(6)^{\circ}, \gamma=90^{\circ}, V=1541.62(17) \AA^{3} . \mathrm{Z}=4, \rho_{\text {calc }}=1.528 \mathrm{~g} / \mathrm{cm}^{3} .13850$ reflections collected, $2747\left[\mathrm{R}_{\text {int }}=0.0636, \mathrm{R}_{\text {sigma }}=0.0396\right]$ independent reflections, $\mathrm{R}_{1}=0.0547, \mathrm{wR}_{2}=0.1379(I>=2 \sigma(I)$, final), $\mathrm{R}_{1}=0.0697, \mathrm{wR}_{2}=0.1472$ (all data), $\mathrm{GOF}=1.027$, and 209 parameters.

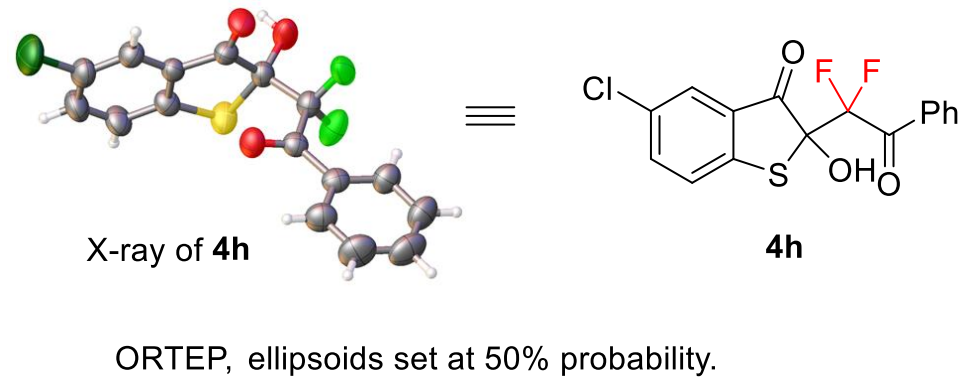

Table S3. Crystal data and structure refinement for $\mathbf{4 h}$.

Identification code

Empirical formula

Formula weight

Temperature/K

Crystal system

Space group

$\mathrm{a} / \AA$

b/ A
$4 h$

$\mathrm{C}_{16} \mathrm{H}_{9} \mathrm{ClF}_{2} \mathrm{O}_{3} \mathrm{~S}$

354.74

292.69(11)

monoclinic

$\mathrm{P} 2{ }_{1} / \mathrm{c}$

15.5043(10)

15.5994(10)

\footnotetext{
${ }^{3}$ Supplementary crystallographic data have been deposited at Cambridge Crystallographic Data Center (CCDC number: 2056189).
} 


\begin{tabular}{|c|c|}
\hline $\mathrm{c} / \AA$ & $6.4142(4)$ \\
\hline$\alpha /^{\circ}$ & 90 \\
\hline$\beta /{ }^{\circ}$ & $96.411(6)$ \\
\hline$\gamma /{ }^{\circ}$ & 90 \\
\hline Volume $/ \AA^{3}$ & $1541.62(17)$ \\
\hline $\mathrm{Z}$ & 4 \\
\hline$\rho_{\text {calc }} \mathrm{g} / \mathrm{cm}^{3}$ & 1.528 \\
\hline$\mu / \mathrm{mm}^{-1}$ & 3.778 \\
\hline $\mathrm{F}(000)$ & 720.0 \\
\hline Crystal size $/ \mathrm{mm}^{3}$ & $0.32 \times 0.16 \times 0.12$ \\
\hline Radiation & $\operatorname{CuK} \alpha(\lambda=1.54184)$ \\
\hline $2 \Theta$ range for data collection $/{ }^{\circ}$ & 8.066 to 134.118 \\
\hline Index ranges & $-18 \leq \mathrm{h} \leq 18,-18 \leq \mathrm{k} \leq 18,-5 \leq 1 \leq 7$ \\
\hline Reflections collected & 13850 \\
\hline Independent reflections & $2747\left[\mathrm{R}_{\mathrm{int}}=0.0636, \mathrm{R}_{\text {sigma }}=0.0396\right]$ \\
\hline Data/restraints/parameters & $2747 / 0 / 209$ \\
\hline Goodness-of-fit on $\mathrm{F}^{2}$ & 1.027 \\
\hline Final $R$ indexes $[\mathrm{I}>=2 \sigma(\mathrm{I})]$ & $\mathrm{R}_{1}=0.0547, \mathrm{wR}_{2}=0.1379$ \\
\hline Final $\mathrm{R}$ indexes [all data] & $\mathrm{R}_{1}=0.0697, \mathrm{wR}_{2}=0.1472$ \\
\hline Largest diff. peak/hole / e $\AA^{-3}$ & $0.53 /-0.23$ \\
\hline
\end{tabular}

Table S4. Fractional Atomic Coordinates $\left(\times 10^{4}\right)$ and Equivalent Isotropic Displacement Parameters $\left(\AA^{2} \times 10^{3}\right)$ for $4 \mathbf{h}$. $U_{\text {eq }}$ is defined as $1 / 3$ of of the trace of the orthogonalised $U_{\text {IJ }}$ tensor.

$\begin{array}{ccccc}\text { Atom } & \boldsymbol{x} & \boldsymbol{y} & \boldsymbol{z} & \mathbf{U}(\mathbf{e q}) \\ \text { S1 } & 7434.0(5) & 4300.0(6) & 7272.2(11) & 60.8(3) \\ \text { C11 } & 3604.2(6) & 3338.9(8) & 4150(2) & 99.3(4) \\ \text { F2 } & 8756.3(12) & 3791.1(11) & 2485(3) & 68.0(5) \\ \text { F1 } & 8998.6(12) & 4599.9(14) & 5252(3) & 72.6(6) \\ \text { O3 } & 7089.8(13) & 4970.4(14) & 2342(3) & 59.0(5) \\ \text { O1 } & 8320.3(14) & 3000.3(14) & 5695(4) & 68.1(6) \\ \text { O2 } & 6982.7(14) & 2974.8(14) & 2142(3) & 65.1(6) \\ & & \text { S5 } & & \end{array}$




$\begin{array}{ccccc}\text { C1 } & 6164.2(18) & 3612.2(17) & 4677(4) & 43.9(6) \\ \text { C10 } & 7875.6(19) & 5004.1(17) & 2583(4) & 45.4(6) \\ \text { C7 } & 7786.7(18) & 3693.3(18) & 5066(4) & 48.0(7) \\ \text { C11 } & 8386(2) & 5667.0(18) & 1626(4) & 49.5(7) \\ \text { C9 } & 8355.4(18) & 4274.5(18) & 3861(4) & 48.2(6) \\ \text { C8 } & 6946.8(18) & 3395.9(17) & 3714(4) & 47.7(7) \\ \text { C6 } & 6339.3(19) & 4066.6(19) & 6533(4) & 49.8(7) \\ \text { C2 } & 5329.5(19) & 3376.1(19) & 3914(5) & 52.2(7) \\ \text { C3 } & 4664(2) & 3612(2) & 5057(5) & 59.7(8) \\ \text { C16 } & 9282(2) & 5651(2) & 1695(5) & 61.0(8) \\ \text { C4 } & 4825(2) & 4067(2) & 6897(6) & 67.4(9) \\ \text { C5 } & 5658(2) & 4291(2) & 7657(5) & 62.7(8) \\ \text { C12 } & 7920(2) & 6334(2) & 567(5) & 66.6(9) \\ \text { C15 } & 9715(3) & 6290(2) & 739(6) & 73.8(10) \\ \text { C13 } & 8359(3) & 6957(3) & -392(7) & 87.7(12) \\ \text { C14 } & 9248(3) & 6940(3) & -292(6) & 86.3(12)\end{array}$

Table S5. Anisotropic Displacement Parameters $\left(\AA^{2} \times 10^{3}\right)$ for $\mathbf{4 h}$. The Anisotropic displacement factor exponent takes the form: $-2 \pi^{2}\left[\mathrm{~h} 2 \mathrm{a}^{* 2} \mathrm{U}_{11}+2 \mathrm{hka} * \mathrm{~b} * \mathrm{U}_{12}+\ldots\right]$.

$\begin{array}{ccccccc}\text { Atom } & \mathbf{U}_{11} & \mathbf{U}_{\mathbf{2 2}} & \mathbf{U}_{\mathbf{3 3}} & \mathbf{U}_{\mathbf{2 3}} & \mathbf{U}_{\mathbf{1 3}} & \mathbf{U}_{12} \\ \mathrm{~S} 1 & 54.6(5) & 82.3(6) & 45.6(4) & -11.0(4) & 6.1(3) & -8.1(4) \\ \mathrm{C} 11 & 45.2(5) & 126.1(9) & 125.1(9) & -6.1(7) & 3.4(5) & -8.1(5) \\ \text { F2 } & 65.3(12) & 61.5(11) & 83.9(12) & 11.0(9) & 38.4(9) & 9.6(9) \\ \text { F1 } & 52.4(11) & 91.7(14) & 70.4(12) & 22.9(10) & -8.2(9) & -20.0(9) \\ \text { O3 } & 46.3(12) & 65.9(13) & 64.7(13) & 9.1(10) & 5.7(9) & 2.0(10) \\ \text { O1 } & 53.2(13) & 64.2(13) & 90.0(17) & 29.1(12) & 21.9(11) & 6.6(11) \\ \text { O2 } & 65.7(14) & 67.1(13) & 65.8(14) & -25.8(11) & 21.8(11) & -6.9(11) \\ \text { C1 } & 45.2(15) & 40.8(14) & 47.1(15) & 2.5(11) & 11.1(12) & 1.9(11) \\ \text { C10 } & 51.0(16) & 46.9(15) & 38.7(14) & -2.8(11) & 6.3(12) & 1.2(13) \\ \text { C7 } & 44.0(15) & 48.6(15) & 52.6(16) & 6.6(12) & 10.9(12) & 3.0(12) \\ \text { C11 } & 61.1(18) & 47.3(15) & 39.9(14) & -2.1(12) & 5.5(12) & -3.0(13) \\ & & & & \text { s6 } & & \end{array}$




$\begin{array}{ccccccc}\text { C9 } & 43.3(15) & 52.7(16) & 48.8(15) & 3.1(13) & 6.1(12) & -1.2(13) \\ \text { C8 } & 50.2(16) & 42.9(14) & 51.5(16) & -3.0(12) & 13.1(13) & -2.2(12) \\ \text { C6 } & 55.1(17) & 53.4(16) & 42.0(15) & -1.1(12) & 10.9(12) & 5.1(13) \\ \text { C2 } & 49.5(17) & 53.9(17) & 53.2(17) & -1.4(13) & 5.6(13) & -3.3(13) \\ \text { C3 } & 44.9(17) & 63.9(19) & 71(2) & 7.2(16) & 7.7(15) & 2.6(14) \\ \text { C16 } & 63(2) & 64.2(19) & 56.3(18) & 9.1(15) & 9.4(15) & -6.7(16) \\ \text { C4 } & 55(2) & 78(2) & 73(2) & -7.7(18) & 23.6(16) & 9.5(17) \\ \text { C5 } & 62(2) & 73(2) & 56.3(18) & -14.3(16) & 18.5(15) & 4.0(16) \\ \text { C12 } & 78(2) & 58.7(19) & 62(2) & 11.4(16) & 5.2(17) & 2.1(17) \\ \text { C15 } & 77(2) & 81(2) & 65(2) & 12.4(19) & 17.1(17) & -21.1(19) \\ \text { C13 } & 109(3) & 73(2) & 80(3) & 29(2) & 7(2) & -3(2) \\ \text { C14 } & 118(4) & 73(2) & 70(2) & 18(2) & 19(2) & -28(2)\end{array}$

Table S6. Bond Lengths for $4 \mathbf{h}$.

\begin{tabular}{|c|c|c|c|c|c|}
\hline Atom & Atom & Length/Å & Atom & Atom & Length/Å \\
\hline $\mathrm{S} 1$ & $\mathrm{C} 7$ & $1.835(3)$ & $\mathrm{C} 7$ & C9 & $1.533(4)$ \\
\hline $\mathrm{S} 1$ & C6 & $1.749(3)$ & $\mathrm{C} 7$ & $\mathrm{C} 8$ & $1.553(4)$ \\
\hline $\mathrm{Cl1}$ & $\mathrm{C} 3$ & $1.734(3)$ & C11 & C16 & $1.385(4)$ \\
\hline $\mathrm{F} 2$ & C9 & $1.363(3)$ & $\mathrm{C} 11$ & $\mathrm{C} 12$ & $1.399(4)$ \\
\hline $\mathrm{F} 1$ & C9 & $1.360(3)$ & C6 & C5 & $1.388(4)$ \\
\hline $\mathrm{O} 3$ & C10 & $1.212(3)$ & $\mathrm{C} 2$ & $\mathrm{C} 3$ & $1.381(4)$ \\
\hline $\mathrm{O} 1$ & $\mathrm{C} 7$ & $1.393(3)$ & $\mathrm{C} 3$ & $\mathrm{C} 4$ & $1.376(5)$ \\
\hline $\mathrm{O} 2$ & $\mathrm{C} 8$ & $1.210(3)$ & C16 & C15 & $1.383(5)$ \\
\hline $\mathrm{C} 1$ & $\mathrm{C} 8$ & $1.461(4)$ & $\mathrm{C} 4$ & C5 & $1.374(5)$ \\
\hline $\mathrm{C} 1$ & C6 & $1.386(4)$ & $\mathrm{C} 12$ & C13 & $1.371(5)$ \\
\hline $\mathrm{C} 1$ & $\mathrm{C} 2$ & $1.382(4)$ & C15 & C14 & $1.373(6)$ \\
\hline C10 & C11 & $1.477(4)$ & C13 & C14 & $1.373(6)$ \\
\hline C10 & C9 & $1.544(4)$ & & & \\
\hline
\end{tabular}

Table S7. Bond Angles for $\mathbf{4 h}$.

Atom Atom Atom Angle $/^{\circ} \quad$ Atom Atom Atom Angle $/^{\circ}$ 


\begin{tabular}{lccc|cccc} 
C6 & S1 & C7 & $92.71(13)$ & F1 & C9 & C7 & $108.1(2)$ \\
C6 & C1 & C8 & $112.9(3)$ & C7 & C9 & C10 & $115.7(2)$ \\
C2 & C1 & C8 & $125.3(3)$ & O2 & C8 & C1 & $126.6(3)$ \\
C2 & C1 & C6 & $121.8(3)$ & O2 & C8 & C7 & $120.9(3)$ \\
O3 & C10 & C11 & $123.7(3)$ & C1 & C8 & C7 & $112.2(2)$ \\
O3 & C10 & C9 & $117.0(2)$ & C1 & C6 & S1 & $115.5(2)$ \\
C11 & C10 & C9 & $119.2(2)$ & C1 & C6 & C5 & $119.2(3)$ \\
O1 & C7 & S1 & $113.3(2)$ & C5 & C6 & S1 & $125.3(2)$ \\
O1 & C7 & C9 & $104.4(2)$ & C3 & C2 & C1 & $117.8(3)$ \\
O1 & C7 & C8 & $111.3(2)$ & C2 & C3 & C11 & $119.5(3)$ \\
C9 & C7 & S1 & $108.75(19)$ & C4 & C3 & C11 & $119.3(3)$ \\
C9 & C7 & C8 & $112.9(2)$ & C4 & C3 & C2 & $121.2(3)$ \\
C8 & C7 & S1 & $106.35(18)$ & C15 & C16 & C11 & $120.5(3)$ \\
C16 & C11 & C10 & $123.8(3)$ & C5 & C4 & C3 & $120.6(3)$ \\
C16 & C11 & C12 & $119.4(3)$ & C4 & C5 & C6 & $119.4(3)$ \\
C12 & C11 & C10 & $116.8(3)$ & C13 & C12 & C11 & $119.3(4)$ \\
F2 & C9 & C10 & $106.9(2)$ & C14 & C15 & C16 & $119.4(4)$ \\
F2 & C9 & C7 & $109.2(2)$ & C12 & C13 & C14 & $120.8(4)$ \\
F1 & C9 & F2 & $106.1(2)$ & C15 & C14 & C13 & $120.6(3)$ \\
F1 & C9 & C10 & $110.3(2)$ & & & &
\end{tabular}

Table S8. Hydrogen Atom Coordinates $\left(\AA \times 10^{4}\right)$ and Isotropic Displacement Parameters $\left(\AA^{2} \times 10^{3}\right)$ for 4h.

$\begin{array}{ccccc}\text { Atom } & \boldsymbol{x} & \boldsymbol{y} & \boldsymbol{z} & \mathbf{U}(\mathbf{e q}) \\ \text { H1 } & 8047.98 & 2652.96 & 6323.93 & 102 \\ \text { H2 } & 5219.47 & 3068.15 & 2671.66 & 63 \\ \text { H16 } & 9595.03 & 5206.48 & 2388.56 & 73 \\ \text { H4 } & 4366.32 & 4223.37 & 7634.51 & 81 \\ \text { H5 } & 5765.06 & 4589.72 & 8912.95 & 75 \\ \text { H12 } & 7318.41 & 6354.31 & 513.57 & 80 \\ \text { H15 } & 10316.81 & 6279.11 & 794.58 & 89 \\ & & \text { s8 } & \end{array}$




$\begin{array}{lrrrr}\text { H13 } & 8050.66 & 7395.9 & -1119.01 & 105 \\ \text { H14 } & 9536.51 & 7374.03 & -927.75 & 104\end{array}$

Data intensity of $\mathbf{5 a}^{2}$ was collected using a 'XtaLAB AFC12 (RINC)' diffractometer at 100.01(13) K. Data collection and reduction were done by using Olex 2 and the structure was solved with the ShelXS structure solution program using direct methods and refined by full-matrix least-squares on $F^{2}$ with anisotropic displacement parameters for non-H atoms using SHELX-97. Hydrogen atoms were added at their geometrically idea positions and refined isotropically. Crystal data for $\mathbf{5 a}: \mathrm{C}_{16} \mathrm{H}_{10} \mathrm{~F}_{2} \mathrm{O}_{3} \mathrm{~S}$, $T=100.01(13) \mathrm{K}$, monoclinic, $\mathrm{P} 2{ }_{1}, a=7.10550(10) \AA, b=11.4391(2) \AA, c=34.0389(6) \AA, \alpha=90^{\circ}$, $\beta=89.638(2)^{\circ}, \gamma=90^{\circ}, V=2766.64(8) \AA^{3} . Z=8, \rho_{\text {calc }}=1.538 \mathrm{~g} / \mathrm{cm}^{3} .26426$ reflections collected, $9020\left[\mathrm{R}_{\text {int }}=0.0969, \mathrm{R}_{\text {sigma }}=0.0720\right]$ independent reflections, $\mathrm{R}_{1}=0.0749, \mathrm{wR}_{2}=0.2019(I>=2 \sigma(I)$, final), $\mathrm{R}_{1}=0.0789, \mathrm{wR}_{2}=0.2053$ (all data), $\mathrm{GOF}=1.066$, and 797 parameters.

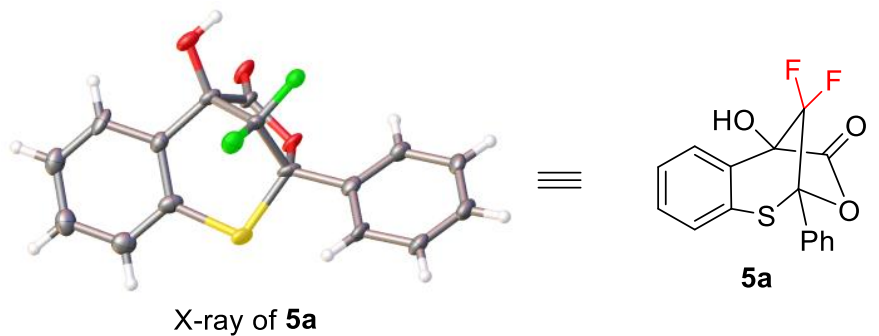

ORTEP, ellipsoids set at $50 \%$ probability

Table S9. Crystal data and structure refinement for 5a.

Identification code

Empirical formula

Formula weight

Temperature/K

Crystal system

Space group

\section{$5 \mathbf{a}$}

$\mathrm{C}_{16} \mathrm{H}_{10} \mathrm{~F}_{2} \mathrm{O}_{3} \mathrm{~S}$

320.30

$100.01(13)$

monoclinic

$\mathrm{P} 21$

2 Supplementary crystallographic data have been deposited at Cambridge Crystallographic Data Center (CCDC number: 2056190). 

$\mathrm{a} / \AA$
$7.10550(10)$
$\mathrm{b} / \AA$
$11.4391(2)$
$\mathrm{c} / \AA$
$34.0389(6)$
$\alpha /{ }^{\circ}$
90
$\beta /^{\circ}$
$89.638(2)$
$\gamma /{ }^{\circ}$
90
Volume $/ \AA^{3}$
2766.64(8)
Z
8
$\rho_{\text {calc }} \mathrm{g} / \mathrm{cm}^{3}$
1.538
$\mu / \mathrm{mm}^{-1}$
2.407
$\mathrm{F}(000)$
1312.0
Crystal size/ $/ \mathrm{mm}^{3}$
$0.32 \times 0.16 \times 0.14$
Radiation
$\operatorname{CuK} \alpha(\lambda=1.54184)$
$2 \Theta$ range for data collection ${ }^{\circ}$
7.792 to 134.156
Index ranges
$-8 \leq \mathrm{h} \leq 4,-13 \leq \mathrm{k} \leq 13,-40 \leq 1 \leq 40$
Reflections collected
26426
Independent reflections
$9020\left[\mathrm{R}_{\text {int }}=0.0969, \mathrm{R}_{\text {sigma }}=0.0720\right]$
Data/restraints/parameters
$9020 / 1 / 797$
Goodness-of-fit on $\mathrm{F}^{2}$
1.066
Final $\mathrm{R}$ indexes $[\mathrm{I}>=2 \sigma(\mathrm{I})]$
$\mathrm{R}_{1}=0.0749, \mathrm{wR}_{2}=0.2019$
Final $\mathrm{R}$ indexes [all data]
$\mathrm{R}_{1}=0.0789, \mathrm{wR}_{2}=0.2053$
Largest diff. peak/hole / e $\AA^{-3}$
$0.56 /-0.70$

Table S10. Fractional Atomic Coordinates $\left(\times 10^{4}\right)$ and Equivalent Isotropic Displacement Parameters $\left(\AA^{2} \times 10^{3}\right)$ for $5 \mathbf{a} . \mathrm{U}_{\text {eq }}$ is defined as $1 / 3$ of of the trace of the orthogonalised $\mathrm{U}_{\mathrm{IJ}}$ tensor.
Atom
$x$
$y$
$z$
$\mathbf{U}(\mathbf{e q})$
C1 254(9)
$-5210(6)$
9352.7(19)
18.9(14) 


\begin{tabular}{|c|c|c|c|c|}
\hline $\mathrm{C} 2$ & $112(9)$ & $-5404(7)$ & $9757.8(19)$ & $21.9(15)$ \\
\hline $\mathrm{C} 3$ & $1460(10)$ & $-6027(7)$ & $9955(2)$ & $25.6(16)$ \\
\hline $\mathrm{C} 4$ & $3011(11)$ & $-6451(7)$ & $9760(2)$ & 29.1(17) \\
\hline $\mathrm{C} 5$ & 3193(11) & $-6299(7)$ & $9355(2)$ & $29.4(18)$ \\
\hline C6 & $1836(9)$ & $-5662(6)$ & $9150.5(19)$ & $20.7(15)$ \\
\hline $\mathrm{C} 7$ & $321(10)$ & $-4521(7)$ & $8521(2)$ & $23.6(16)$ \\
\hline $\mathrm{C} 8$ & $-265(10)$ & $-3303(7)$ & 9055.2(19) & $22.0(15)$ \\
\hline C9 & $-1222(9)$ & $-4494(7)$ & $9144.9(18)$ & $19.5(15)$ \\
\hline $\mathrm{C} 10$ & $-1495(9)$ & $-4913(6)$ & 8729.3(18) & $16.5(13)$ \\
\hline $\mathrm{C} 11$ & $180(10)$ & $-4357(7)$ & $8083(2)$ & $24.0(16)$ \\
\hline $\mathrm{C} 12$ & $653(9)$ & $-5245(7)$ & $7821.5(17)$ & $22.5(14)$ \\
\hline $\mathrm{C} 13$ & 463(9) & $-5089(7)$ & 7412.9(17) & $23.3(15)$ \\
\hline C14 & $-263(9)$ & $-4011(7)$ & 7275.7(19) & $23.3(15)$ \\
\hline $\mathrm{C} 15$ & $-783(9)$ & $-3164(7)$ & 7534.1(18) & $24.0(15)$ \\
\hline $\mathrm{C} 16$ & $-570(9)$ & $-3323(7)$ & $7940.0(17)$ & $22.1(14)$ \\
\hline $\mathrm{F} 1$ & $-1828(6)$ & $-6076(4)$ & $8690.0(11)$ & $23.7(9)$ \\
\hline $\mathrm{F} 2$ & $-3025(5)$ & $-4368(4)$ & 8566.3(11) & 23.3(9) \\
\hline O1 & $-2836(7)$ & $-4421(5)$ & 9377.7(13) & $24.3(11)$ \\
\hline $\mathrm{O} 2$ & $-244(7)$ & $-2448(5)$ & $9247.7(14)$ & $26.5(12)$ \\
\hline $\mathrm{O} 3$ & $628(7)$ & $-3398(5)$ & 8702.1(13) & $21.6(11)$ \\
\hline $\mathrm{S} 1$ & $2241(2)$ & $-5484.9(18)$ & $8640.9(5)$ & $26.2(5)$ \\
\hline $\mathrm{C} 17$ & $-1721(9)$ & $-898(7)$ & $5852.6(19)$ & $24.7(16)$ \\
\hline $\mathrm{C} 18$ & $-3071(11)$ & $-1552(7)$ & $5649(2)$ & $27.4(17)$ \\
\hline C19 & $-2941(11)$ & $-1673(7)$ & $5241(2)$ & $31.1(18)$ \\
\hline $\mathrm{C} 20$ & $-1467(10)$ & $-1174(7)$ & $5039(2)$ & $27.8(17)$ \\
\hline $\mathrm{C} 21$ & $-130(9)$ & $-532(7)$ & $5232.0(18)$ & $21.7(15)$ \\
\hline $\mathrm{C} 22$ & $-200(10)$ & $-397(7)$ & $5640.1(19)$ & $21.5(15)$ \\
\hline $\mathrm{C} 23$ & $1230(10)$ & $378(7)$ & $5841.0(18)$ & $20.9(15)$ \\
\hline $\mathrm{C} 24$ & $170(10)$ & $1532(7)$ & $5925.4(18)$ & $22.9(16)$ \\
\hline $\mathrm{C} 25$ & $-255(10)$ & $315(7)$ & $6466(2)$ & $25.2(16)$ \\
\hline
\end{tabular}




\begin{tabular}{|c|c|c|c|c|}
\hline $\mathrm{C} 26$ & 1592(9) & $2(7)$ & $6259.9(18)$ & $20.0(14)$ \\
\hline $\mathrm{C} 27$ & $-152(9)$ & $525(7)$ & $6903.8(19)$ & $21.0(15)$ \\
\hline $\mathrm{C} 28$ & $-752(9)$ & $1556(7)$ & $7070.0(18)$ & $25.2(15)$ \\
\hline $\mathrm{C} 29$ & $-601(9)$ & $1719(7)$ & $7476.4(18)$ & $21.6(14)$ \\
\hline C30 & $200(10)$ & $890(7)$ & 7711.0(19) & $24.5(16)$ \\
\hline C31 & $756(9)$ & $-164(7)$ & 7548.7(17) & $26.2(16)$ \\
\hline C32 & $604(10)$ & $-356(7)$ & 7144.7(17) & $25.0(15)$ \\
\hline F3 & $2052(6)$ & $-1146(4)$ & $6298.2(11)$ & $24.6(9)$ \\
\hline $\mathrm{F} 4$ & $3054(5)$ & $622(4)$ & 6412.1(11) & $25.6(10)$ \\
\hline $\mathrm{O} 4$ & $-726(7)$ & $1407(5)$ & $6277.1(13)$ & $22.1(11)$ \\
\hline O5 & $67(8)$ & $2377(5)$ & $5728.6(14)$ & $30.1(13)$ \\
\hline O6 & $2812(7)$ & $494(5)$ & $5601.0(14)$ & $25.6(12)$ \\
\hline $\mathrm{S} 2$ & $-2040(2)$ & $-759.0(19)$ & $6364.1(5)$ & $26.1(5)$ \\
\hline C33 & $4700(10)$ & $5199(7)$ & $5754.3(19)$ & $21.5(15)$ \\
\hline C34 & $4796(10)$ & $5566(8)$ & $5366(2)$ & $29.8(18)$ \\
\hline $\mathrm{C} 35$ & $3388(11)$ & $6263(7)$ & $5215(2)$ & $27.3(16)$ \\
\hline C36 & 1887(11) & $6608(8)$ & $5440(2)$ & $34.2(18)$ \\
\hline C37 & $1755(11)$ & $6231(8)$ & $5830(2)$ & $28.9(17)$ \\
\hline C38 & $3156(10)$ & $5528(7)$ & $5992(2)$ & $25.9(16)$ \\
\hline C39 & $4787(9)$ & $4109(7)$ & $6543.6(18)$ & $18.3(14)$ \\
\hline $\mathrm{C} 40$ & $5236(10)$ & $3161(7)$ & $5940.7(18)$ & $20.3(15)$ \\
\hline $\mathrm{C} 41$ & $6195(10)$ & $4368(7)$ & $5909.0(18)$ & $21.8(15)$ \\
\hline $\mathrm{C} 42$ & $6566(9)$ & $4609(7)$ & 6343.6(19) & $19.8(14)$ \\
\hline $\mathrm{C} 43$ & 4914(9) & $3767(7)$ & $6970(2)$ & $22.4(15)$ \\
\hline $\mathrm{C} 44$ & $5603(9)$ & $4566(7)$ & $7234.3(17)$ & $21.8(14)$ \\
\hline $\mathrm{C} 45$ & $5676(9)$ & $4295(7)$ & 7636.2(17) & $24.4(16)$ \\
\hline $\mathrm{C} 46$ & $5079(9)$ & $3224(7)$ & $7760(2)$ & $23.5(16)$ \\
\hline $\mathrm{C} 47$ & $4344(9)$ & $2413(7)$ & $7497.4(18)$ & $24.2(15)$ \\
\hline $\mathrm{C} 48$ & $4297(9)$ & $2675(7)$ & 7093.9(18) & $22.3(15)$ \\
\hline F5 & $8093(5)$ & $3982(4)$ & $6461.7(11)$ & 23.1(9) \\
\hline
\end{tabular}




\begin{tabular}{|c|c|c|c|c|}
\hline F6 & $6935(5)$ & $5736(4)$ & $6431.2(11)$ & $25.1(9)$ \\
\hline $\mathrm{O} 7$ & $5154(7)$ & $2416(5)$ & $5707.5(13)$ & $28.9(12)$ \\
\hline $\mathrm{O} 8$ & $4421(6)$ & $3077(5)$ & $6306.0(13)$ & $20.5(11)$ \\
\hline O9 & $7800(7)$ & $4366(5)$ & $5670.0(13)$ & $24.4(11)$ \\
\hline $\mathrm{S} 3$ & $2873(2)$ & $5148.7(17)$ & $6488.2(5)$ & $24.6(4)$ \\
\hline $\mathrm{C} 49$ & $-3012(10)$ & $517(7)$ & $9008(2)$ & $25.3(16)$ \\
\hline C50 & $-1623(11)$ & $1224(8)$ & $9163(2)$ & $29.7(17)$ \\
\hline C51 & $-1826(11)$ & $1680(8)$ & $9540(2)$ & $32.0(18)$ \\
\hline C52 & $-3396(11)$ & $1397(7)$ & $9761(2)$ & $30.0(17)$ \\
\hline C53 & $-4795(10)$ & $709(7)$ & $9609(2)$ & $26.6(17)$ \\
\hline C54 & $-4640(10)$ & $255(7)$ & $9232(2)$ & $22.6(15)$ \\
\hline C55 & $-6159(9)$ & $-551(7)$ & $9079.2(18)$ & $20.2(15)$ \\
\hline C56 & $-5287(9)$ & $-1797(6)$ & $9063.7(18)$ & $18.5(14)$ \\
\hline C57 & $-4663(9)$ & $-913(7)$ & $8452.4(19)$ & $20.4(14)$ \\
\hline C58 & $-6426(9)$ & $-335(7)$ & $8633.4(18)$ & $18.9(14)$ \\
\hline C59 & $-4834(9)$ & $-1283(7)$ & $8031(2)$ & $22.5(15)$ \\
\hline C60 & $-5625(9)$ & $-2368(7)$ & 7943.1(18) & $23.9(15)$ \\
\hline C61 & $-5870(9)$ & $-2700(7)$ & 7555.9(19) & $24.4(15)$ \\
\hline C62 & $-5358(10)$ & $-1950(8)$ & $7257(2)$ & $28.2(17)$ \\
\hline C63 & $-4549(9)$ & $-890(7)$ & $7336.5(18)$ & $25.3(15)$ \\
\hline C64 & $-4297(9)$ & $-544(7)$ & $7731.8(17)$ & $23.9(15)$ \\
\hline F7 & $-7975(5)$ & $-916(4)$ & $8513.0(11)$ & 21.5(9) \\
\hline $\mathrm{F} 8$ & $-6656(6)$ & 789(4) & $8529.8(11)$ & $25.7(9)$ \\
\hline $\mathrm{O} 10$ & $-4446(6)$ & $-1937(5)$ & $8702.2(13)$ & $20.8(11)$ \\
\hline $\mathrm{O} 11$ & $-5290(7)$ & $-2516(5)$ & 9303.2(13) & $27.6(12)$ \\
\hline $\mathrm{O} 12$ & $-7788(7)$ & $-487(5)$ & $9303.8(13)$ & $25.8(12)$ \\
\hline $\mathrm{S} 4$ & $-2641(2)$ & $37.8(18)$ & $8520.8(5)$ & $26.9(5)$ \\
\hline
\end{tabular}

Table S11. Anisotropic Displacement Parameters $\left(\AA^{2} \times 10^{3}\right)$ for 5a. The Anisotropic displacement factor exponent takes the form: $-2 \pi^{2}\left[h^{2} a^{* 2} U_{11}+2 h k a * b * U_{12}+\ldots\right]$.
Atom
$\mathrm{U}_{11}$
$\mathrm{U}_{22}$
$\mathbf{U}_{33} \quad \mathbf{U}_{23}$
$\mathrm{U}_{13}$
$\mathrm{U}_{12}$ 


\begin{tabular}{|c|c|c|c|c|c|c|}
\hline $\mathrm{C} 1$ & $23(3)$ & $18(4)$ & $16(3)$ & $-1(3)$ & $3(2)$ & $0(3)$ \\
\hline $\mathrm{C} 2$ & $27(3)$ & $26(4)$ & $13(3)$ & $4(3)$ & $8(3)$ & $-6(3)$ \\
\hline $\mathrm{C} 3$ & $39(4)$ & $27(4)$ & 11(3) & $5(3)$ & $0(3)$ & $-3(3)$ \\
\hline $\mathrm{C} 4$ & $35(4)$ & $32(5)$ & $20(4)$ & $6(3)$ & $-1(3)$ & 2(3) \\
\hline $\mathrm{C} 5$ & $37(4)$ & $38(5)$ & $13(3)$ & $-5(3)$ & $6(3)$ & $14(3)$ \\
\hline $\mathrm{C} 6$ & $22(3)$ & $24(4)$ & $16(3)$ & $-4(3)$ & $3(3)$ & $5(3)$ \\
\hline $\mathrm{C} 7$ & $29(4)$ & $29(4)$ & 13(3) & $-8(3)$ & $9(3)$ & 2(3) \\
\hline $\mathrm{C} 8$ & $31(4)$ & $27(4)$ & $8(3)$ & 1(3) & $9(3)$ & $3(3)$ \\
\hline C9 & $23(3)$ & $29(4)$ & $6(3)$ & $3(3)$ & $8(2)$ & $3(3)$ \\
\hline $\mathrm{C} 10$ & 20(3) & $20(4)$ & $10(3)$ & $0(3)$ & $4(2)$ & $6(3)$ \\
\hline $\mathrm{C} 11$ & $25(4)$ & $35(4)$ & $12(3)$ & $-4(3)$ & $6(3)$ & $-1(3)$ \\
\hline $\mathrm{C} 12$ & $24(3)$ & $32(4)$ & $12(3)$ & $-1(3)$ & $6(2)$ & 1(3) \\
\hline $\mathrm{C} 13$ & $28(3)$ & $30(4)$ & $12(3)$ & $-1(3)$ & $7(2)$ & $-3(3)$ \\
\hline $\mathrm{C} 14$ & 21(3) & $36(4)$ & $13(3)$ & $4(3)$ & 2(3) & $-2(3)$ \\
\hline $\mathrm{C} 15$ & $26(3)$ & $34(4)$ & $12(3)$ & $6(3)$ & $3(3)$ & 1(3) \\
\hline $\mathrm{C} 16$ & $25(3)$ & $28(4)$ & 13(3) & $-1(3)$ & $6(2)$ & $-2(3)$ \\
\hline $\mathrm{F} 1$ & $34(2)$ & $20(2)$ & 17.2(19) & $0.7(16)$ & $2.7(16)$ & $-6.2(17)$ \\
\hline $\mathrm{F} 2$ & 21.1(19) & $34(3)$ & $14.6(18)$ & $7.2(17)$ & $2.0(15)$ & $2.2(17)$ \\
\hline $\mathrm{O} 1$ & $24(2)$ & $33(3)$ & $16(2)$ & $1(2)$ & 11.7(19) & $6(2)$ \\
\hline $\mathrm{O} 2$ & $38(3)$ & $25(3)$ & $16(2)$ & $-5(2)$ & $12(2)$ & $-2(2)$ \\
\hline $\mathrm{O} 3$ & $31(3)$ & $26(3)$ & $8(2)$ & $-4.4(19)$ & 8.6(18) & $-1(2)$ \\
\hline $\mathrm{S} 1$ & $26.0(9)$ & $40.8(13)$ & $11.8(7)$ & $-2.2(7)$ & $4.8(6)$ & $11.3(7)$ \\
\hline $\mathrm{C} 17$ & 20(3) & $38(5)$ & $16(3)$ & $3(3)$ & $5(3)$ & $-3(3)$ \\
\hline $\mathrm{C} 18$ & $35(4)$ & $35(5)$ & $12(3)$ & $5(3)$ & $5(3)$ & $-7(3)$ \\
\hline C19 & $37(4)$ & $36(5)$ & $20(4)$ & $-9(3)$ & $-2(3)$ & $-7(3)$ \\
\hline C20 & $34(4)$ & $39(5)$ & 11(3) & $-4(3)$ & $0(3)$ & $9(3)$ \\
\hline $\mathrm{C} 21$ & $25(3)$ & $29(4)$ & 11(3) & $-4(3)$ & $7(2)$ & 2(3) \\
\hline $\mathrm{C} 22$ & $27(4)$ & $25(4)$ & $13(3)$ & $0(3)$ & 1(3) & $4(3)$ \\
\hline $\mathrm{C} 23$ & $26(4)$ & $30(4)$ & 7(3) & $-1(3)$ & $9(3)$ & $-6(3)$ \\
\hline C24 & $33(4)$ & $27(4)$ & $8(3)$ & $4(3)$ & $8(3)$ & $-8(3)$ \\
\hline
\end{tabular}




\begin{tabular}{|c|c|c|c|c|c|c|}
\hline $\mathrm{C} 25$ & $34(4)$ & $29(4)$ & $13(3)$ & $2(3)$ & $6(3)$ & $-2(3)$ \\
\hline $\mathrm{C} 26$ & $22(3)$ & $27(4)$ & $11(3)$ & $-1(3)$ & $4(2)$ & $-5(3)$ \\
\hline $\mathrm{C} 27$ & $23(3)$ & $32(4)$ & $8(3)$ & $9(3)$ & $4(2)$ & $-1(3)$ \\
\hline $\mathrm{C} 28$ & $25(4)$ & $37(5)$ & $13(3)$ & $1(3)$ & $2(2)$ & 1(3) \\
\hline $\mathrm{C} 29$ & $25(3)$ & $26(4)$ & $14(3)$ & $-4(3)$ & $4(2)$ & $1(3)$ \\
\hline $\mathrm{C} 30$ & $27(4)$ & $37(5)$ & $9(3)$ & $-1(3)$ & $6(3)$ & $-5(3)$ \\
\hline C31 & $25(3)$ & $45(5)$ & $8(3)$ & $6(3)$ & $-2(2)$ & $1(3)$ \\
\hline $\mathrm{C} 32$ & $33(4)$ & $29(4)$ & $13(3)$ & $-2(3)$ & $5(3)$ & $8(3)$ \\
\hline F3 & $36(2)$ & $22(2)$ & 15.3(19) & $-1.2(16)$ & $1.6(16)$ & $3.9(18)$ \\
\hline $\mathrm{F} 4$ & $25(2)$ & $36(3)$ & 15.9(19) & $-9.6(18)$ & $3.2(15)$ & $-4.9(18)$ \\
\hline $\mathrm{O} 4$ & $34(3)$ & $25(3)$ & $8(2)$ & $6(2)$ & $8.2(18)$ & $4(2)$ \\
\hline O5 & $43(3)$ & $32(3)$ & $15(2)$ & $3(2)$ & $12(2)$ & $3(2)$ \\
\hline O6 & $23(2)$ & $37(3)$ & $16(2)$ & $-4(2)$ & $12.4(19)$ & $-8(2)$ \\
\hline $\mathrm{S} 2$ & 27.7(9) & $39.8(13)$ & $10.7(7)$ & $2.8(7)$ & $3.6(6)$ & $-8.6(7)$ \\
\hline $\mathrm{C} 33$ & $25(3)$ & $26(4)$ & $13(3)$ & $4(3)$ & $3(3)$ & $-7(3)$ \\
\hline $\mathrm{C} 34$ & $26(4)$ & $46(5)$ & $18(3)$ & $6(3)$ & $5(3)$ & $-17(3)$ \\
\hline $\mathrm{C} 35$ & $35(4)$ & $26(4)$ & $21(3)$ & $5(3)$ & $-2(3)$ & $-8(3)$ \\
\hline C36 & $39(4)$ & $39(5)$ & $25(4)$ & $2(4)$ & $-10(3)$ & $-5(4)$ \\
\hline $\mathrm{C} 37$ & $28(4)$ & $39(5)$ & $20(3)$ & $-7(3)$ & $1(3)$ & $1(3)$ \\
\hline C38 & $26(4)$ & $37(5)$ & $15(3)$ & $-4(3)$ & $4(3)$ & $1(3)$ \\
\hline C39 & $21(3)$ & $22(4)$ & $12(3)$ & $-8(3)$ & $6(2)$ & $-6(3)$ \\
\hline $\mathrm{C} 40$ & $29(4)$ & $25(4)$ & $7(3)$ & $-6(3)$ & $7(3)$ & $0(3)$ \\
\hline $\mathrm{C} 41$ & $24(3)$ & $30(4)$ & $11(3)$ & $-5(3)$ & $7(2)$ & $0(3)$ \\
\hline $\mathrm{C} 42$ & $22(3)$ & $22(4)$ & $16(3)$ & $3(3)$ & $1(3)$ & $-2(3)$ \\
\hline $\mathrm{C} 43$ & $18(3)$ & $35(4)$ & $14(3)$ & $0(3)$ & $5(3)$ & $-2(3)$ \\
\hline $\mathrm{C} 44$ & $25(3)$ & $31(4)$ & $9(3)$ & $2(3)$ & $4(2)$ & $-7(3)$ \\
\hline $\mathrm{C} 45$ & $27(4)$ & $36(5)$ & $10(3)$ & $-1(3)$ & $3(2)$ & $0(3)$ \\
\hline $\mathrm{C} 46$ & $24(4)$ & $36(4)$ & $11(3)$ & $5(3)$ & $2(3)$ & $3(3)$ \\
\hline $\mathrm{C} 47$ & $24(4)$ & $32(4)$ & $16(3)$ & $8(3)$ & $7(3)$ & $1(3)$ \\
\hline $\mathrm{C} 48$ & $25(3)$ & $30(4)$ & $12(3)$ & $6(3)$ & $2(2)$ & $-5(3)$ \\
\hline
\end{tabular}




\begin{tabular}{|c|c|c|c|c|c|c|}
\hline F5 & $20.2(19)$ & $32(2)$ & $17.4(18)$ & $7.4(17)$ & $3.1(15)$ & $0.0(17)$ \\
\hline F6 & $30(2)$ & $31(2)$ & $14.2(19)$ & $-0.3(17)$ & $2.3(16)$ & $-9.3(18)$ \\
\hline $\mathrm{O} 7$ & $43(3)$ & $32(3)$ & $12(2)$ & $1(2)$ & $11(2)$ & $-7(2)$ \\
\hline O8 & $25(2)$ & $25(3)$ & $11(2)$ & $-1(2)$ & $8.3(18)$ & $-5(2)$ \\
\hline O9 & $28(3)$ & $29(3)$ & $16(2)$ & $0(2)$ & $13.2(19)$ & $0(2)$ \\
\hline $\mathrm{S} 3$ & $25.1(9)$ & $37.3(11)$ & $11.4(7)$ & $-1.6(7)$ & $4.7(6)$ & $4.7(8)$ \\
\hline C49 & $23(4)$ & $34(4)$ & $19(3)$ & $8(3)$ & $3(3)$ & $1(3)$ \\
\hline C50 & $30(4)$ & $34(5)$ & $25(4)$ & $6(3)$ & $-2(3)$ & $-2(3)$ \\
\hline C51 & $36(4)$ & $33(5)$ & $27(4)$ & $-4(4)$ & $-8(3)$ & $1(3)$ \\
\hline C52 & $39(4)$ & $32(4)$ & $19(3)$ & $-2(3)$ & $-5(3)$ & $6(3)$ \\
\hline C53 & $29(4)$ & $36(5)$ & $15(3)$ & $-6(3)$ & $1(3)$ & $17(3)$ \\
\hline C54 & $25(3)$ & $26(4)$ & $17(3)$ & $-3(3)$ & $2(3)$ & $3(3)$ \\
\hline C55 & $20(3)$ & $27(4)$ & $13(3)$ & $5(3)$ & $6(2)$ & $3(3)$ \\
\hline C56 & $22(3)$ & $22(4)$ & $12(3)$ & $-1(3)$ & $7(3)$ & $0(3)$ \\
\hline $\mathrm{C} 57$ & $16(3)$ & $30(4)$ & $15(3)$ & $8(3)$ & $6(2)$ & $1(3)$ \\
\hline C58 & $21(3)$ & $21(4)$ & $15(3)$ & $0(3)$ & $5(2)$ & $-5(3)$ \\
\hline C59 & $19(3)$ & $30(4)$ & $18(3)$ & $-3(3)$ & $3(3)$ & $5(3)$ \\
\hline C60 & $25(4)$ & $32(4)$ & $14(3)$ & $2(3)$ & $3(2)$ & $1(3)$ \\
\hline C61 & $22(3)$ & $27(4)$ & $23(3)$ & $-10(3)$ & $4(3)$ & $1(3)$ \\
\hline C62 & $26(4)$ & $44(5)$ & $15(3)$ & $-7(3)$ & $3(3)$ & $0(3)$ \\
\hline C63 & $29(4)$ & $31(4)$ & $16(3)$ & $-2(3)$ & $7(3)$ & $4(3)$ \\
\hline C64 & $24(3)$ & $38(5)$ & $10(3)$ & $-3(3)$ & $5(2)$ & $0(3)$ \\
\hline F7 & $22.0(19)$ & $25(2)$ & $16.9(18)$ & $-0.8(17)$ & $3.1(15)$ & $1.4(17)$ \\
\hline F8 & $33(2)$ & $26(2)$ & $17.6(19)$ & $5.2(17)$ & $1.0(16)$ & $2.3(18)$ \\
\hline $\mathrm{O} 10$ & $26(2)$ & $25(3)$ & $11(2)$ & $2(2)$ & $8.8(18)$ & $4(2)$ \\
\hline O11 & $39(3)$ & $33(3)$ & $11(2)$ & $0(2)$ & $7(2)$ & $6(2)$ \\
\hline $\mathrm{O} 12$ & $27(3)$ & $35(3)$ & $16(2)$ & $2(2)$ & $8.6(19)$ & $3(2)$ \\
\hline $\mathrm{S} 4$ & $23.9(8)$ & $43.7(12)$ & $13.0(7)$ & $0.0(8)$ & $4.4(6)$ & $-8.4(8)$ \\
\hline
\end{tabular}


Table S12. Bond Lengths for 5 a.

\begin{tabular}{|c|c|c|c|c|c|}
\hline Atom & Atom & Length/Å & Atom & Atom & Length/Å \\
\hline $\mathrm{C} 1$ & $\mathrm{C} 2$ & $1.400(9)$ & $\mathrm{C} 33$ & $\mathrm{C} 34$ & $1.387(9)$ \\
\hline $\mathrm{C} 1$ & C6 & $1.412(9)$ & $\mathrm{C} 33$ & C38 & $1.411(9)$ \\
\hline $\mathrm{C} 1$ & C9 & $1.511(9)$ & $\mathrm{C} 33$ & $\mathrm{C} 41$ & $1.521(10)$ \\
\hline $\mathrm{C} 2$ & $\mathrm{C} 3$ & $1.372(10)$ & C34 & C35 & $1.381(11)$ \\
\hline $\mathrm{C} 3$ & $\mathrm{C} 4$ & $1.371(11)$ & $\mathrm{C} 35$ & $\mathrm{C} 36$ & $1.367(11)$ \\
\hline $\mathrm{C} 4$ & $\mathrm{C} 5$ & $1.395(10)$ & C36 & C37 & $1.396(11)$ \\
\hline $\mathrm{C} 5$ & C6 & $1.397(10)$ & C37 & C38 & $1.396(11)$ \\
\hline C6 & S1 & $1.768(6)$ & C38 & S3 & $1.754(7)$ \\
\hline $\mathrm{C} 7$ & $\mathrm{C} 10$ & $1.536(9)$ & C39 & $\mathrm{C} 42$ & $1.542(9)$ \\
\hline $\mathrm{C} 7$ & $\mathrm{C} 11$ & $1.506(9)$ & C39 & $\mathrm{C} 43$ & $1.507(9)$ \\
\hline $\mathrm{C} 7$ & $\mathrm{O} 3$ & $1.442(9)$ & C39 & O8 & $1.456(8)$ \\
\hline C7 & S1 & $1.804(8)$ & C39 & S3 & $1.817(7)$ \\
\hline $\mathrm{C} 8$ & C9 & $1.552(10)$ & $\mathrm{C} 40$ & $\mathrm{C} 41$ & $1.544(10)$ \\
\hline $\mathrm{C} 8$ & $\mathrm{O} 2$ & $1.177(9)$ & $\mathrm{C} 40$ & $\mathrm{O} 7$ & $1.166(9)$ \\
\hline $\mathrm{C} 8$ & $\mathrm{O} 3$ & $1.360(7)$ & $\mathrm{C} 40$ & O8 & $1.372(7)$ \\
\hline C9 & $\mathrm{C} 10$ & $1.508(9)$ & C41 & C42 & $1.529(8)$ \\
\hline $\mathrm{C} 9$ & $\mathrm{O} 1$ & $1.392(7)$ & $\mathrm{C} 41$ & O9 & $1.396(7)$ \\
\hline $\mathrm{C} 10$ & $\mathrm{~F} 1$ & $1.358(8)$ & $\mathrm{C} 42$ & F5 & $1.363(8)$ \\
\hline $\mathrm{C} 10$ & $\mathrm{~F} 2$ & $1.373(7)$ & C42 & F6 & $1.350(9)$ \\
\hline $\mathrm{C} 11$ & $\mathrm{C} 12$ & $1.390(10)$ & $\mathrm{C} 43$ & $\mathrm{C} 44$ & $1.375(10)$ \\
\hline C11 & C16 & $1.387(11)$ & C43 & $\mathrm{C} 48$ & $1.388(11)$ \\
\hline $\mathrm{C} 12$ & $\mathrm{C} 13$ & $1.410(8)$ & $\mathrm{C} 44$ & $\mathrm{C} 45$ & $1.404(8)$ \\
\hline $\mathrm{C} 13$ & $\mathrm{C} 14$ & $1.417(11)$ & $\mathrm{C} 45$ & $\mathrm{C} 46$ & $1.363(11)$ \\
\hline $\mathrm{C} 14$ & $\mathrm{C} 15$ & $1.358(10)$ & $\mathrm{C} 46$ & $\mathrm{C} 47$ & $1.393(10)$ \\
\hline $\mathrm{C} 15$ & C16 & $1.403(8)$ & $\mathrm{C} 47$ & $\mathrm{C} 48$ & $1.407(8)$ \\
\hline $\mathrm{C} 17$ & $\mathrm{C} 18$ & $1.404(10)$ & C49 & C50 & $1.385(11)$ \\
\hline $\mathrm{C} 17$ & $\mathrm{C} 22$ & $1.418(9)$ & C49 & C54 & $1.413(9)$ \\
\hline $\mathrm{C} 17$ & S2 & $1.762(7)$ & C49 & S4 & $1.764(7)$ \\
\hline
\end{tabular}




\begin{tabular}{|c|c|c|c|c|c|}
\hline $\mathrm{C} 18$ & C19 & $1.396(10)$ & $\mathrm{C} 50$ & C51 & $1.391(11)$ \\
\hline C19 & $\mathrm{C} 20$ & $1.374(11)$ & C51 & C52 & $1.381(11)$ \\
\hline $\mathrm{C} 20$ & $\mathrm{C} 21$ & $1.372(10)$ & C52 & C53 & $1.373(11)$ \\
\hline $\mathrm{C} 21$ & $\mathrm{C} 22$ & $1.398(9)$ & $\mathrm{C} 53$ & C54 & $1.388(9)$ \\
\hline $\mathrm{C} 22$ & $\mathrm{C} 23$ & $1.515(10)$ & $\mathrm{C} 54$ & C55 & $1.513(10)$ \\
\hline $\mathrm{C} 23$ & $\mathrm{C} 24$ & $1.546(11)$ & C55 & C56 & $1.555(10)$ \\
\hline $\mathrm{C} 23$ & $\mathrm{C} 26$ & $1.513(9)$ & C55 & C58 & $1.550(8)$ \\
\hline $\mathrm{C} 23$ & O6 & $1.392(7)$ & C55 & O12 & $1.386(7)$ \\
\hline $\mathrm{C} 24$ & $\mathrm{O} 4$ & $1.360(7)$ & C56 & O10 & $1.374(7)$ \\
\hline $\mathrm{C} 24$ & O5 & $1.179(9)$ & C56 & O11 & $1.158(9)$ \\
\hline $\mathrm{C} 25$ & C26 & $1.527(9)$ & $\mathrm{C} 57$ & C58 & $1.542(9)$ \\
\hline $\mathrm{C} 25$ & $\mathrm{C} 27$ & $1.510(9)$ & $\mathrm{C} 57$ & C59 & $1.502(9)$ \\
\hline $\mathrm{C} 25$ & $\mathrm{O} 4$ & $1.446(9)$ & $\mathrm{C} 57$ & $\mathrm{O} 10$ & $1.456(8)$ \\
\hline $\mathrm{C} 25$ & $\mathrm{~S} 2$ & $1.801(8)$ & $\mathrm{C} 57$ & $\mathrm{~S} 4$ & $1.818(7)$ \\
\hline $\mathrm{C} 26$ & $\mathrm{~F} 3$ & $1.359(9)$ & C58 & F7 & $1.352(8)$ \\
\hline $\mathrm{C} 26$ & $\mathrm{~F} 4$ & $1.363(8)$ & C58 & F8 & $1.343(9)$ \\
\hline $\mathrm{C} 27$ & C28 & $1.375(11)$ & C59 & C60 & $1.396(11)$ \\
\hline $\mathrm{C} 27$ & C32 & $1.408(10)$ & C59 & C64 & $1.376(10)$ \\
\hline $\mathrm{C} 28$ & $\mathrm{C} 29$ & $1.401(8)$ & C60 & C61 & $1.384(9)$ \\
\hline $\mathrm{C} 29$ & C30 & $1.366(10)$ & C61 & C62 & $1.379(11)$ \\
\hline $\mathrm{C} 30$ & C31 & $1.382(11)$ & C62 & C63 & $1.369(11)$ \\
\hline C31 & C32 & $1.397(8)$ & C63 & C64 & $1.415(8)$ \\
\hline
\end{tabular}

Table S13. Bond Angles for 5a.

\begin{tabular}{cccc|cccc} 
Atom & Atom & Atom & Angle $^{\circ}$ & Atom & Atom & Atom & Angle $^{\circ}$ \\
C2 & C1 & C6 & $118.3(6)$ & C34 & C33 & C38 & $119.9(7)$ \\
C2 & C1 & C9 & $120.1(6)$ & C34 & C33 & C41 & $119.2(6)$ \\
C6 & C1 & C9 & $121.5(5)$ & C38 & C33 & C41 & $120.7(6)$ \\
C3 & C2 & C1 & $121.1(6)$ & C35 & C34 & C33 & $119.9(7)$ \\
C4 & C3 & C2 & $120.6(6)$ & C36 & C35 & C34 & $121.5(7)$
\end{tabular}




\begin{tabular}{|c|c|c|c|c|c|c|c|}
\hline $\mathrm{C} 3$ & $\mathrm{C} 4$ & C5 & $120.2(7)$ & C35 & C36 & C37 & $119.4(8)$ \\
\hline $\mathrm{C} 4$ & $\mathrm{C} 5$ & C6 & 119.8(7) & $\mathrm{C} 38$ & $\mathrm{C} 37$ & $\mathrm{C} 36$ & $120.6(7)$ \\
\hline $\mathrm{C} 1$ & C6 & $\mathrm{S} 1$ & 124.1(5) & $\mathrm{C} 33$ & $\mathrm{C} 38$ & S3 & $124.8(6)$ \\
\hline $\mathrm{C} 5$ & C6 & $\mathrm{C} 1$ & 119.9(6) & $\mathrm{C} 37$ & C 38 & $\mathrm{C} 33$ & $118.7(6)$ \\
\hline $\mathrm{C} 5$ & C6 & $\mathrm{S} 1$ & $116.0(5)$ & C37 & $\mathrm{C} 38$ & S3 & $116.5(5)$ \\
\hline $\mathrm{C} 10$ & $\mathrm{C} 7$ & $\mathrm{~S} 1$ & $110.5(5)$ & $\mathrm{C} 42$ & C39 & S3 & $108.9(5)$ \\
\hline $\mathrm{C} 11$ & $\mathrm{C} 7$ & $\mathrm{C} 10$ & $115.7(6)$ & $\mathrm{C} 43$ & C39 & $\mathrm{C} 42$ & $117.9(5)$ \\
\hline $\mathrm{C} 11$ & $\mathrm{C} 7$ & S1 & $110.8(5)$ & $\mathrm{C} 43$ & C39 & S3 & $108.6(4)$ \\
\hline $\mathrm{O} 3$ & $\mathrm{C} 7$ & $\mathrm{C} 10$ & $101.0(5)$ & $\mathrm{O} 8$ & C39 & $\mathrm{C} 42$ & $101.8(5)$ \\
\hline $\mathrm{O} 3$ & $\mathrm{C} 7$ & $\mathrm{C} 11$ & 109.0(6) & $\mathrm{O} 8$ & C 39 & $\mathrm{C} 43$ & $109.7(6)$ \\
\hline $\mathrm{O} 3$ & $\mathrm{C} 7$ & S1 & $109.3(5)$ & $\mathrm{O} 8$ & C 39 & S3 & $109.7(4)$ \\
\hline $\mathrm{O} 2$ & $\mathrm{C} 8$ & $\mathrm{C} 9$ & $128.8(6)$ & $\mathrm{O} 7$ & $\mathrm{C} 40$ & $\mathrm{C} 41$ & $129.1(6)$ \\
\hline $\mathrm{O} 2$ & $\mathrm{C} 8$ & $\mathrm{O} 3$ & $123.4(7)$ & $\mathrm{O} 7$ & $\mathrm{C} 40$ & O8 & $122.9(7)$ \\
\hline $\mathrm{O} 3$ & $\mathrm{C} 8$ & $\mathrm{C} 9$ & 107.7(6) & $\mathrm{O} 8$ & $\mathrm{C} 40$ & $\mathrm{C} 41$ & $108.1(5)$ \\
\hline $\mathrm{C} 1$ & $\mathrm{C} 9$ & $\mathrm{C} 8$ & $105.3(6)$ & $\mathrm{C} 33$ & $\mathrm{C} 41$ & $\mathrm{C} 40$ & $105.9(6)$ \\
\hline $\mathrm{C} 10$ & $\mathrm{C} 9$ & $\mathrm{C} 1$ & 111.1(6) & $\mathrm{C} 33$ & $\mathrm{C} 41$ & $\mathrm{C} 42$ & $110.3(6)$ \\
\hline $\mathrm{C} 10$ & C9 & $\mathrm{C} 8$ & $98.8(5)$ & $\mathrm{C} 42$ & $\mathrm{C} 41$ & $\mathrm{C} 40$ & $99.9(5)$ \\
\hline $\mathrm{O} 1$ & C9 & $\mathrm{C} 1$ & 109.7(5) & O9 & $\mathrm{C} 41$ & $\mathrm{C} 33$ & $111.6(6)$ \\
\hline $\mathrm{O} 1$ & $\mathrm{C} 9$ & $\mathrm{C} 8$ & $114.7(6)$ & O9 & $\mathrm{C} 41$ & $\mathrm{C} 40$ & $113.4(6)$ \\
\hline $\mathrm{O} 1$ & $\mathrm{C} 9$ & $\mathrm{C} 10$ & $116.3(6)$ & O9 & $\mathrm{C} 41$ & $\mathrm{C} 42$ & $114.8(6)$ \\
\hline C9 & $\mathrm{C} 10$ & $\mathrm{C} 7$ & 103.2(5) & C41 & $\mathrm{C} 42$ & C39 & $102.4(5)$ \\
\hline $\mathrm{F} 1$ & $\mathrm{C} 10$ & $\mathrm{C} 7$ & $112.8(5)$ & F5 & $\mathrm{C} 42$ & C39 & $109.1(6)$ \\
\hline F1 & $\mathrm{C} 10$ & $\mathrm{C} 9$ & $115.3(6)$ & F5 & $\mathrm{C} 42$ & $\mathrm{C} 41$ & $109.5(6)$ \\
\hline F1 & $\mathrm{C} 10$ & $\mathrm{~F} 2$ & $105.4(5)$ & F6 & $\mathrm{C} 42$ & C39 & $114.6(6)$ \\
\hline $\mathrm{F} 2$ & $\mathrm{C} 10$ & $\mathrm{C} 7$ & $110.2(5)$ & F6 & $\mathrm{C} 42$ & $\mathrm{C} 41$ & $114.9(6)$ \\
\hline $\mathrm{F} 2$ & $\mathrm{C} 10$ & C9 & $110.0(5)$ & F6 & $\mathrm{C} 42$ & F5 & $106.3(5)$ \\
\hline $\mathrm{C} 12$ & $\mathrm{C} 11$ & $\mathrm{C} 7$ & 121.7(7) & C44 & $\mathrm{C} 43$ & C39 & $118.8(7)$ \\
\hline $\mathrm{C} 16$ & C11 & $\mathrm{C} 7$ & 118.7(6) & C44 & $\mathrm{C} 43$ & $\mathrm{C} 48$ & $120.9(6)$ \\
\hline $\mathrm{C} 16$ & $\mathrm{C} 11$ & $\mathrm{C} 12$ & $119.4(6)$ & $\mathrm{C} 48$ & $\mathrm{C} 43$ & C39 & $120.3(6)$ \\
\hline $\mathrm{C} 11$ & $\mathrm{C} 12$ & $\mathrm{C} 13$ & $121.0(7)$ & $\mathrm{C} 43$ & C44 & $\mathrm{C} 45$ & $120.4(7)$ \\
\hline
\end{tabular}




\begin{tabular}{|c|c|c|c|c|c|c|c|}
\hline $\mathrm{C} 12$ & $\mathrm{C} 13$ & $\mathrm{C} 14$ & 118.2(6) & C46 & $\mathrm{C} 45$ & $\mathrm{C} 44$ & $119.2(7)$ \\
\hline $\mathrm{C} 15$ & $\mathrm{C} 14$ & $\mathrm{C} 13$ & $120.4(6)$ & $\mathrm{C} 45$ & $\mathrm{C} 46$ & $\mathrm{C} 47$ & $121.1(6)$ \\
\hline $\mathrm{C} 14$ & $\mathrm{C} 15$ & $\mathrm{C} 16$ & $121.0(7)$ & C46 & C47 & $\mathrm{C} 48$ & $119.8(7)$ \\
\hline $\mathrm{C} 11$ & $\mathrm{C} 16$ & $\mathrm{C} 15$ & $120.0(6)$ & $\mathrm{C} 43$ & $\mathrm{C} 48$ & $\mathrm{C} 47$ & $118.6(7)$ \\
\hline $\mathrm{C} 8$ & $\mathrm{O} 3$ & $\mathrm{C} 7$ & $112.3(5)$ & $\mathrm{C} 40$ & O8 & C39 & $111.7(5)$ \\
\hline C6 & S1 & $\mathrm{C} 7$ & $100.0(3)$ & C38 & S3 & C39 & $100.4(3)$ \\
\hline $\mathrm{C} 18$ & $\mathrm{C} 17$ & $\mathrm{C} 22$ & $119.0(6)$ & C50 & C49 & C54 & $120.0(7)$ \\
\hline $\mathrm{C} 18$ & $\mathrm{C} 17$ & S2 & $116.9(5)$ & C50 & C49 & $\mathrm{S} 4$ & $116.0(5)$ \\
\hline $\mathrm{C} 22$ & $\mathrm{C} 17$ & S2 & $124.1(5)$ & C54 & C49 & S4 & $123.9(6)$ \\
\hline $\mathrm{C} 19$ & $\mathrm{C} 18$ & $\mathrm{C} 17$ & $120.2(7)$ & C49 & C50 & C51 & $120.1(7)$ \\
\hline $\mathrm{C} 20$ & C19 & $\mathrm{C} 18$ & $120.2(7)$ & C52 & C51 & C50 & $119.6(8)$ \\
\hline $\mathrm{C} 21$ & $\mathrm{C} 20$ & C19 & $120.6(6)$ & C53 & C52 & C51 & $120.9(7)$ \\
\hline $\mathrm{C} 20$ & $\mathrm{C} 21$ & $\mathrm{C} 22$ & $121.0(7)$ & C52 & $\mathrm{C} 53$ & C54 & $120.7(7)$ \\
\hline $\mathrm{C} 17$ & $\mathrm{C} 22$ & $\mathrm{C} 23$ & $121.2(6)$ & C49 & C54 & C55 & $121.8(6)$ \\
\hline $\mathrm{C} 21$ & $\mathrm{C} 22$ & $\mathrm{C} 17$ & $118.9(7)$ & C53 & C54 & $\mathrm{C} 49$ & $118.6(7)$ \\
\hline $\mathrm{C} 21$ & $\mathrm{C} 22$ & $\mathrm{C} 23$ & $119.6(6)$ & C53 & C54 & C55 & $119.5(6)$ \\
\hline $\mathrm{C} 22$ & $\mathrm{C} 23$ & $\mathrm{C} 24$ & $104.8(6)$ & C54 & C55 & C56 & $106.6(5)$ \\
\hline $\mathrm{C} 26$ & $\mathrm{C} 23$ & $\mathrm{C} 22$ & $112.2(6)$ & C54 & C55 & C58 & $109.3(5)$ \\
\hline $\mathrm{C} 26$ & $\mathrm{C} 23$ & $\mathrm{C} 24$ & $98.8(5)$ & C58 & C55 & C56 & $99.5(5)$ \\
\hline O6 & $\mathrm{C} 23$ & $\mathrm{C} 22$ & $109.4(5)$ & $\mathrm{O} 12$ & C55 & C54 & $112.0(6)$ \\
\hline O6 & $\mathrm{C} 23$ & $\mathrm{C} 24$ & 114.7(6) & $\mathrm{O} 12$ & C55 & C56 & $113.5(6)$ \\
\hline O6 & $\mathrm{C} 23$ & $\mathrm{C} 26$ & $116.0(6)$ & $\mathrm{O} 12$ & C55 & C58 & $115.1(5)$ \\
\hline $\mathrm{O} 4$ & $\mathrm{C} 24$ & $\mathrm{C} 23$ & $107.4(6)$ & $\mathrm{O} 10$ & C56 & C55 & $107.9(5)$ \\
\hline O5 & $\mathrm{C} 24$ & $\mathrm{C} 23$ & $128.8(6)$ & O11 & C56 & C55 & $128.9(6)$ \\
\hline O5 & $\mathrm{C} 24$ & $\mathrm{O} 4$ & $123.8(7)$ & O11 & C56 & $\mathrm{O} 10$ & $123.1(6)$ \\
\hline $\mathrm{C} 26$ & $\mathrm{C} 25$ & $\mathrm{~S} 2$ & $110.8(5)$ & C58 & C57 & S4 & $109.5(5)$ \\
\hline $\mathrm{C} 27$ & $\mathrm{C} 25$ & $\mathrm{C} 26$ & $116.4(6)$ & C59 & C57 & C58 & $115.6(5)$ \\
\hline $\mathrm{C} 27$ & $\mathrm{C} 25$ & $\mathrm{~S} 2$ & $109.7(5)$ & C59 & C57 & $\mathrm{S} 4$ & 111.1(5) \\
\hline $\mathrm{O} 4$ & $\mathrm{C} 25$ & $\mathrm{C} 26$ & $101.4(5)$ & $\mathrm{O} 10$ & C57 & C58 & $101.6(5)$ \\
\hline $\mathrm{O} 4$ & $\mathrm{C} 25$ & $\mathrm{C} 27$ & $108.3(6)$ & O10 & C57 & C59 & $109.9(6)$ \\
\hline
\end{tabular}




\begin{tabular}{|c|c|c|c|c|c|c|c|}
\hline $\mathrm{O} 4$ & $\mathrm{C} 25$ & $\mathrm{~S} 2$ & $109.7(5)$ & $\mathrm{O} 10$ & C57 & S4 & $108.6(4)$ \\
\hline $\mathrm{C} 23$ & $\mathrm{C} 26$ & $\mathrm{C} 25$ & $102.5(6)$ & C57 & C58 & C55 & $102.6(5)$ \\
\hline F3 & $\mathrm{C} 26$ & $\mathrm{C} 23$ & $114.0(6)$ & F7 & C58 & C55 & $108.9(5)$ \\
\hline F3 & $\mathrm{C} 26$ & $\mathrm{C} 25$ & $112.9(6)$ & F7 & C58 & C57 & $109.2(6)$ \\
\hline F3 & $\mathrm{C} 26$ & $\mathrm{~F} 4$ & $106.4(5)$ & F8 & C58 & C55 & $115.2(6)$ \\
\hline $\mathrm{F} 4$ & $\mathrm{C} 26$ & $\mathrm{C} 23$ & $110.2(6)$ & F8 & C58 & C57 & $113.9(5)$ \\
\hline $\mathrm{F} 4$ & $\mathrm{C} 26$ & $\mathrm{C} 25$ & $111.0(6)$ & F8 & C58 & F7 & $106.9(6)$ \\
\hline $\mathrm{C} 28$ & $\mathrm{C} 27$ & $\mathrm{C} 25$ & $121.7(6)$ & C60 & C59 & C57 & $119.4(6)$ \\
\hline $\mathrm{C} 28$ & $\mathrm{C} 27$ & C32 & $119.5(6)$ & C64 & C59 & C57 & $120.7(7)$ \\
\hline $\mathrm{C} 32$ & $\mathrm{C} 27$ & $\mathrm{C} 25$ & $118.8(7)$ & C64 & C59 & C60 & $119.9(7)$ \\
\hline $\mathrm{C} 27$ & C28 & C29 & $119.7(7)$ & C61 & C60 & C59 & $120.1(7)$ \\
\hline $\mathrm{C} 30$ & C29 & $\mathrm{C} 28$ & $121.3(7)$ & C62 & C61 & C60 & $119.9(7)$ \\
\hline $\mathrm{C} 29$ & $\mathrm{C} 30$ & C31 & $119.4(6)$ & C63 & C62 & C61 & $120.9(7)$ \\
\hline $\mathrm{C} 30$ & C31 & C32 & $120.4(7)$ & C62 & C63 & C64 & $119.5(7)$ \\
\hline C31 & C32 & $\mathrm{C} 27$ & $119.5(7)$ & C59 & C64 & C63 & $119.7(7)$ \\
\hline $\mathrm{C} 24$ & $\mathrm{O} 4$ & $\mathrm{C} 25$ & $112.0(5)$ & C56 & O10 & C57 & $112.5(5)$ \\
\hline C17 & $\mathrm{S} 2$ & $\mathrm{C} 25$ & $99.6(3)$ & $\mathrm{C} 49$ & $\mathrm{~S} 4$ & C57 & 101.1(3) \\
\hline
\end{tabular}

Table S14. Hydrogen Atom Coordinates $\left(\AA \times 10^{4}\right)$ and Isotropic Displacement Parameters $\left(\AA^{2} \times 10^{3}\right)$ for $5 \mathbf{a}$.

$\begin{array}{ccccc}\text { Atom } & \boldsymbol{x} & \boldsymbol{y} & \boldsymbol{z} & \mathbf{U}(\mathbf{e q}) \\ \text { H2 } & -913.45 & -5105.22 & 9896.03 & 26 \\ \text { H3 } & 1320.89 & -6163.5 & 10222.5 & 31 \\ \text { H4 } & 3944.68 & -6840.93 & 9898.02 & 35 \\ \text { H5 } & 4213.91 & -6620.44 & 9220.9 & 35 \\ \text { H12 } & 1100.24 & -5952.76 & 7917.65 & 27 \\ \text { H13 } & 805.73 & -5677.12 & 7238 & 28 \\ \text { H14 } & -384.5 & -3882.55 & 7007.28 & 28 \\ \text { H15 } & -1287.69 & -2467.85 & 7440.53 & 29 \\ \text { H16 } & -930.68 & -2734.49 & 8113.44 & 27 \\ \text { H1 } & -3636.67 & -4035.52 & 9263.46 & 36\end{array}$




\begin{tabular}{|c|c|c|c|}
\hline H18 & -4054.24 & -1906.58 & 5785.44 \\
\hline H19 & -3854.84 & -2091.63 & 5106.52 \\
\hline $\mathrm{H} 20$ & -1374.54 & -1272.81 & 4768.15 \\
\hline $\mathrm{H} 21$ & 837.02 & -181.74 & 5089.38 \\
\hline $\mathrm{H} 28$ & -1255.28 & 2143.04 & 6913.33 \\
\hline $\mathrm{H} 29$ & -1055.56 & 2404.83 & 7588.88 \\
\hline H30 & 369.46 & 1032.7 & 7977.34 \\
\hline H31 & 1235.09 & -748.58 & 7709.47 \\
\hline H32 & 999.47 & -1060.78 & 7035.79 \\
\hline H6 & 3519.09 & 986.56 & 5693.95 \\
\hline H34 & 5806.64 & 5343.36 & 5208.21 \\
\hline H35 & 3463.18 & 6504.09 & 4954.71 \\
\hline H36 & 963 & 7088.2 & 5334.98 \\
\hline H37 & 724.58 & 6451.17 & 5982.47 \\
\hline $\mathrm{H} 44$ & 6023.41 & 5291.06 & 7146.28 \\
\hline $\mathrm{H} 45$ & 6126.76 & 4839.31 & 7815.41 \\
\hline $\mathrm{H} 46$ & 5163.99 & 3030.97 & 8025.22 \\
\hline $\mathrm{H} 47$ & 3886.03 & 1700.09 & 7588.34 \\
\hline $\mathrm{H} 48$ & 3862.81 & 2128.32 & 6913.7 \\
\hline H9 & 8360.9 & 3745.11 & 5696.51 \\
\hline $\mathrm{H} 50$ & -551.46 & 1395.3 & 9015.85 \\
\hline H51 & -909.69 & 2172.72 & 9642.36 \\
\hline H52 & -3506.89 & 1675.3 & 10017.08 \\
\hline H53 & -5858.64 & 544.93 & 9759.5 \\
\hline H60 & -5988.42 & -2868.44 & 8145.09 \\
\hline H61 & -6379.16 & -3428.14 & 7497.49 \\
\hline H62 & -5564.88 & -2166.25 & 6997.28 \\
\hline H63 & -4167.2 & -402.23 & 7132.44 \\
\hline H64 & -3772.15 & 180.67 & 7789.12 \\
\hline $\mathrm{H} 12 \mathrm{~A}$ & -8502.28 & -1017.3 & 9240.91 \\
\hline
\end{tabular}


Data intensity of $\mathbf{5 i}^{3}$ was collected using a 'XtaLAB AFC12 (RINC)' diffractometer at 99.94(18) K. Data collection and reduction were done by using Olex2 and the structure was solved with the ShelXS structure solution program using direct methods and refined by full-matrix least-squares on $F^{2}$ with anisotropic displacement parameters for non-H atoms using SHELX-97. Hydrogen atoms were added at their geometrically idea positions and refined isotropically. Crystal data for $5 \mathbf{i}: \mathrm{C}_{16} \mathrm{H}_{9} \mathrm{BrF}_{2} \mathrm{O}_{3} \mathrm{~S}$, $T=99.94(18) \mathrm{K}$, triclinic, $\mathrm{P}-1, a=7.4596(2) \AA, b=9.2236(3) \AA, c=11.0671(5) \AA, \alpha=106.586(3)^{\circ}, \beta$ $=90.288(3)^{\circ}, \gamma=96.906(3)^{\circ}, V=723.87(5) \AA^{3} . Z=2, \rho_{\text {calc }}=1.832 \mathrm{~g} / \mathrm{cm}^{3} .5574$ reflections collected, $2507\left[\mathrm{R}_{\mathrm{int}}=0.0310, \mathrm{R}_{\text {sigma }}=0.0296\right]$ independent reflections, $\mathrm{R}_{1}=0.0346, \mathrm{wR}_{2}=0.0886(I>=2 \sigma(I)$, final), $\mathrm{R}_{1}=0.0355, \mathrm{wR}_{2}=0.0892$ (all data), $\mathrm{GOF}=1.081$, and 209 parameters.

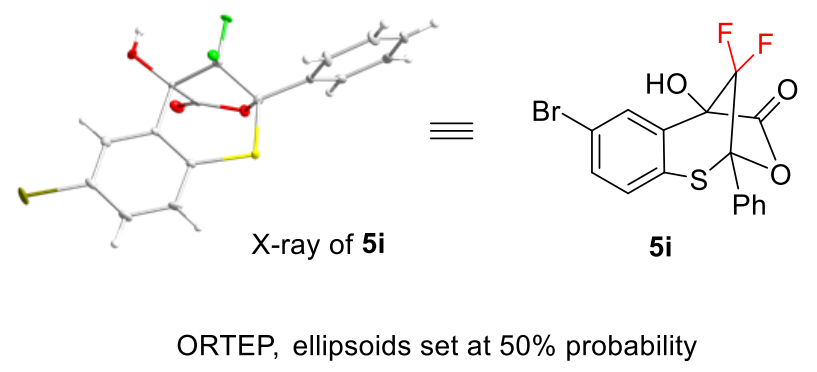

Table S15. Crystal data and structure refinement for $\mathbf{5 i}$.

\begin{tabular}{|c|c|}
\hline Identification code & $5 \mathbf{i}$ \\
\hline Empirical formula & $\mathrm{C}_{16} \mathrm{H}_{9} \mathrm{BrF}_{2} \mathrm{O}_{3} \mathrm{~S}$ \\
\hline Formula weight & 399.20 \\
\hline Temperature/K & $99.94(18)$ \\
\hline Crystal system & triclinic \\
\hline Space group & $\mathrm{P}-1$ \\
\hline $\mathrm{a} / \AA$ & $7.4596(2)$ \\
\hline $\mathrm{b} / \AA$ & $9.2236(3)$ \\
\hline $\mathrm{c} / \AA$ & $11.0671(5)$ \\
\hline$\alpha /^{\circ}$ & $106.586(3)$ \\
\hline
\end{tabular}

3 Supplementary crystallographic data have been deposited at Cambridge Crystallographic Data Center (CCDC number: 2056188). 


\begin{tabular}{|c|c|}
\hline$\beta /{ }^{\circ}$ & $90.288(3)$ \\
\hline$\gamma /{ }^{\circ}$ & $96.906(3)$ \\
\hline Volume $/ \AA^{3}$ & $723.87(5)$ \\
\hline $\mathrm{Z}$ & 2 \\
\hline$\rho_{\text {calc }} \mathrm{g} / \mathrm{cm}^{3}$ & 1.832 \\
\hline$\mu / \mathrm{mm}^{-1}$ & 5.560 \\
\hline $\mathrm{F}(000)$ & 396.0 \\
\hline Crystal size $/ \mathrm{mm}^{3}$ & $0.32 \times 0.28 \times 0.26$ \\
\hline Radiation & $\mathrm{CuK} \alpha(\lambda=1.54184)$ \\
\hline $2 \Theta$ range for data collection $/^{\circ}$ & 10.088 to 134.106 \\
\hline Index ranges & $-5 \leq \mathrm{h} \leq 8,-11 \leq \mathrm{k} \leq 10,-13 \leq 1 \leq 13$ \\
\hline Reflections collected & 5574 \\
\hline Independent reflections & $2507\left[\mathrm{R}_{\mathrm{int}}=0.0310, \mathrm{R}_{\text {sigma }}=0.0296\right]$ \\
\hline Data/restraints/parameters & $2507 / 18 / 209$ \\
\hline Goodness-of-fit on $\mathrm{F}^{2}$ & 1.081 \\
\hline Final $R$ indexes $[\mathrm{I}>=2 \sigma(\mathrm{I})]$ & $\mathrm{R}_{1}=0.0346, \mathrm{w} \mathrm{R}_{2}=0.0886$ \\
\hline Final $\mathrm{R}$ indexes [all data] & $\mathrm{R}_{1}=0.0355, \mathrm{wR}_{2}=0.0892$ \\
\hline Largest diff. peak/hole / e $\AA^{-3}$ & $0.72 /-0.89$ \\
\hline
\end{tabular}

Table S16. Fractional Atomic Coordinates $\left(\times 10^{4}\right)$ and Equivalent Isotropic Displacement Parameters $\left(\AA^{2} \times 10^{3}\right)$ for $5 \mathbf{i}$. $U_{\text {eq }}$ is defined as $1 / 3$ of of the trace of the orthogonalised $U_{\text {IJ }}$ tensor.

$\begin{array}{ccccc}\text { Atom } & \boldsymbol{x} & \boldsymbol{y} & \boldsymbol{z} & \mathbf{U}(\mathbf{e q}) \\ \mathrm{Br} 1 & 792.9(3) & 1686.4(3) & 4759.9(2) & 17.63(13) \\ \text { S1 } & 4156.8(7) & 8141.5(6) & 4103.3(5) & 9.25(16) \\ \text { F1 } & 4021.6(18) & 6304.8(15) & 400.8(12) & 10.0(3) \\ \text { F2 } & 5559.9(18) & 5876.1(15) & 1914.5(12) & 9.7(3) \\ \text { O3 } & 1907(2) & 3939.8(18) & 950.3(15) & 10.7(4) \\ \text { O1 } & 1784(2) & 7922.9(18) & 2199.0(15) & 9.5(3) \\ & & \text { S24 } & & \end{array}$




$\begin{array}{lllll}\text { O2 } & -629(2) & 6175(2) & 1500.2(16) & 12.5(4) \\ \text { C11 } & 3141(3) & 6353(3) & 4197(2) & 7.2(4) \\ \text { C5 } & 3980(3) & 10353(3) & 1772(2) & 11.2(5) \\ \text { C15 } & 1660(3) & 3777(3) & 3341(2) & 9.0(5) \\ \text { C6 } & 4810(3) & 9240(3) & 2103(2) & 8.8(5) \\ \text { C10 } & 2377(3) & 5160(3) & 3164(2) & 6.5(4) \\ \text { C8 } & 934(3) & 6475(3) & 1819(2) & 8.4(5) \\ \text { C16 } & 4010(3) & 6331(3) & 1641(2) & 6.6(4) \\ \text { C9 } & 2302(3) & 5360(3) & 1843(2) & 6.7(4) \\ \text { C7 } & 3722(3) & 7946(3) & 2456(2) & 7.5(5) \\ \text { C14 } & 1721(3) & 3591(3) & 4543(2) & 9.7(5) \\ \text { C13 } & 2455(3) & 4765(3) & 5577(2) & 11.2(5) \\ \text { C1 } & 6687(3) & 9296(3) & 2092(2) & 13.5(5) \\ \text { C3 } & 6883(4) & 11546(3) & 1381(2) & 14.3(5) \\ \text { C4 } & 5020(4) & 11510(3) & 1417(2) & 12.7(5) \\ \text { C2 } & 7715(3) & 10446(3) & 1723(2) & 15.1(5) \\ \text { C12 } & 3157(3) & 6144(3) & 5400(2) & 10.4(5)\end{array}$

Table S17. Anisotropic Displacement Parameters $\left(\AA^{2} \times 10^{3}\right)$ for 5 i. The Anisotropic displacement factor exponent takes the form: $-2 \pi^{2}\left[h^{2} a^{* 2} U_{11}+2 h k a * b * U_{12}+\ldots\right]$.

$\begin{array}{ccccccc}\text { Atom } & \mathbf{U}_{11} & \mathbf{U}_{22} & \mathbf{U}_{\mathbf{3 3}} & \mathbf{U}_{23} & \mathbf{U}_{13} & \mathbf{U}_{12} \\ \text { Br1 } & 19.21(19) & 15.53(19) & 21.93(19) & 14.24(12) & 0.33(12) & -4.71(12) \\ \text { S1 } & 12.7(3) & 7.7(3) & 4.6(3) & 0.1(2) & -1.7(2) & -5.3(2) \\ \text { F1 } & 13.5(7) & 13.5(7) & 3.2(6) & 3.8(5) & 1.2(5) & -1.4(5) \\ \text { F2 } & 6.3(6) & 12.7(7) & 9.8(6) & 3.4(5) & 0.0(5) & 0.6(5) \\ \text { O3 } & 16.1(8) & 8.9(8) & 3.6(7) & -0.5(6) & -4.0(6) & -5.5(6) \\ \text { O1 } & 5.7(8) & 10.4(8) & 11.9(8) & 3.4(6) & -0.5(6) & -0.8(6) \\ \text { O2 } & 5.8(8) & 21.2(9) & 10.6(8) & 6.4(7) & -2.6(6) & -2.4(7) \\ \text { C11 } & 5.7(11) & 9.5(11) & 6.0(10) & 2.1(9) & 0.4(8) & -0.2(8) \\ \text { C5 } & 12.2(12) & 13.2(12) & 7.0(11) & 1.7(9) & 1.5(9) & -0.4(9) \\ \text { C15 } & 5.5(11) & 11.0(11) & 9.7(11) & 2.3(9) & 0.6(8) & -0.8(8)\end{array}$




$\begin{array}{ccccccc}\text { C6 } & 13.1(12) & 7.5(11) & 4.6(10) & 1.4(8) & 0.0(8) & -2.8(9) \\ \text { C10 } & 3.1(10) & 10.4(11) & 5.8(10) & 2.9(8) & 0.0(8) & -1.1(8) \\ \text { C8 } & 10.0(10) & 11.3(10) & 3.8(9) & 3.4(8) & 1.4(8) & -1.7(8) \\ \text { C16 } & 5.8(11) & 9.3(11) & 4.2(10) & 1.8(8) & -1.3(8) & 0.1(8) \\ \text { C9 } & 8.8(11) & 6.0(10) & 3.6(10) & 0.2(8) & -0.4(8) & -2.7(8) \\ \text { C7 } & 4.9(10) & 9.1(11) & 7.2(11) & 1.0(9) & -1.9(8) & -0.7(8) \\ \text { C14 } & 5.6(11) & 14.6(12) & 11.8(11) & 8.0(9) & 2.5(9) & 2.0(9) \\ \text { C13 } & 9.1(12) & 18.1(12) & 8.6(11) & 7.6(9) & 0.7(9) & 1.1(9) \\ \text { C1 } & 11.9(12) & 12.3(12) & 17.1(12) & 7.6(10) & -2.4(9) & -4.1(9) \\ \text { C3 } & 20.8(13) & 11.4(12) & 8.0(11) & 2.5(9) & -0.3(9) & -8.7(9) \\ \text { C4 } & 21.7(13) & 9.7(11) & 6.0(10) & 2.0(9) & 0.4(9) & 0.2(9) \\ \text { C2 } & 9.1(12) & 17.9(13) & 16.9(12) & 6.0(10) & -0.7(9) & -6.1(9) \\ \text { C12 } & 8.5(11) & 17.6(13) & 4.9(11) & 3.1(9) & -0.2(8) & 1.3(9)\end{array}$

Table S18. Bond Lengths for $\mathbf{5 i}$.

\begin{tabular}{|c|c|c|c|c|c|}
\hline Atom & Atom & Length/Å & Atom & Atom & Length/Å \\
\hline $\mathrm{Br} 1$ & C14 & $1.890(3)$ & C15 & $\mathrm{C} 10$ & $1.389(3)$ \\
\hline $\mathrm{S} 1$ & C11 & $1.762(2)$ & $\mathrm{C} 15$ & C14 & $1.390(3)$ \\
\hline S1 & $\mathrm{C} 7$ & $1.804(2)$ & C6 & $\mathrm{C} 7$ & $1.508(3)$ \\
\hline $\mathrm{F} 1$ & C16 & $1.366(3)$ & C6 & $\mathrm{C} 1$ & $1.395(4)$ \\
\hline $\mathrm{F} 2$ & C16 & $1.338(3)$ & $\mathrm{C} 10$ & C9 & $1.527(3)$ \\
\hline $\mathrm{O} 3$ & C9 & $1.395(3)$ & $\mathrm{C} 8$ & C9 & $1.538(3)$ \\
\hline $\mathrm{O} 1$ & $\mathrm{C} 8$ & $1.355(3)$ & $\mathrm{C} 16$ & C9 & $1.523(3)$ \\
\hline $\mathrm{O} 1$ & $\mathrm{C} 7$ & $1.468(3)$ & $\mathrm{C} 16$ & $\mathrm{C} 7$ & $1.546(3)$ \\
\hline $\mathrm{O} 2$ & $\mathrm{C} 8$ & $1.193(3)$ & C14 & C13 & $1.388(4)$ \\
\hline $\mathrm{C} 11$ & C10 & $1.402(3)$ & $\mathrm{C} 13$ & $\mathrm{C} 12$ & $1.384(4)$ \\
\hline $\mathrm{C} 11$ & C12 & $1.399(3)$ & $\mathrm{C} 1$ & $\mathrm{C} 2$ & $1.391(4)$ \\
\hline C5 & C6 & $1.391(3)$ & $\mathrm{C} 3$ & $\mathrm{C} 4$ & $1.387(4)$ \\
\hline $\mathrm{C} 5$ & $\mathrm{C} 4$ & $1.393(4)$ & C3 & $\mathrm{C} 2$ & $1.388(4)$ \\
\hline
\end{tabular}


Table S19. Bond Angles for 5i.

\begin{tabular}{cccc|cccc} 
Atom & Atom & Atom & Angle ${ }^{\circ}$ & Atom & Atom & Atom & Angle $^{\circ}$ \\
C11 & S1 & C7 & $100.63(11)$ & O3 & C9 & C10 & $109.74(19)$ \\
C8 & O1 & C7 & $111.13(17)$ & O3 & C9 & C8 & $113.94(18)$ \\
C10 & C11 & S1 & $124.71(19)$ & O3 & C9 & C16 & $115.68(18)$ \\
C12 & C11 & S1 & $115.70(18)$ & C10 & C9 & C8 & $108.31(17)$ \\
C12 & C11 & C10 & $119.6(2)$ & C16 & C9 & C10 & $109.97(18)$ \\
C6 & C5 & C4 & $120.0(2)$ & C16 & C9 & C8 & $98.59(18)$ \\
C10 & C15 & C14 & $119.4(2)$ & O1 & C7 & S1 & $110.31(15)$ \\
C5 & C6 & C7 & $121.4(2)$ & O1 & C7 & C6 & $110.80(18)$ \\
C5 & C6 & C1 & $119.9(2)$ & O1 & C7 & C16 & $100.49(17)$ \\
C1 & C6 & C7 & $118.7(2)$ & C6 & C7 & S1 & $109.47(15)$ \\
C11 & C10 & C9 & $120.9(2)$ & C6 & C7 & C16 & $115.16(19)$ \\
C15 & C10 & C11 & $119.9(2)$ & C16 & C7 & S1 & $110.31(16)$ \\
C15 & C10 & C9 & $119.2(2)$ & C15 & C14 & Br1 & $118.87(18)$ \\
O1 & C8 & C9 & $109.31(19)$ & C13 & C14 & Br1 & $119.63(19)$ \\
O2 & C8 & O1 & $122.9(2)$ & C13 & C14 & C15 & $121.5(2)$ \\
O2 & C8 & C9 & $127.7(2)$ & C12 & C13 & C14 & $119.0(2)$ \\
F1 & C16 & C9 & $108.41(17)$ & C2 & C1 & C6 & $119.7(2)$ \\
F1 & C16 & C7 & $109.08(18)$ & C4 & C3 & C2 & $120.0(2)$ \\
F2 & C16 & F1 & $107.38(17)$ & C3 & C4 & C5 & $120.0(2)$ \\
F2 & C16 & C9 & $115.10(19)$ & C3 & C2 & C1 & $120.3(2)$ \\
F2 & C16 & C7 & $114.09(18)$ & C13 & C12 & C11 & $120.7(2)$ \\
C9 & C16 & C7 & $102.55(17)$ & & & & \\
\hline
\end{tabular}

Table S20. Hydrogen Atom Coordinates $\left(\AA \times 10^{4}\right)$ and Isotropic Displacement Parameters $\left(\AA^{2} \times 10^{3}\right)$ for $5 i$.

$\begin{array}{ccccc}\text { Atom } & \boldsymbol{x} & \boldsymbol{y} & \boldsymbol{z} & \mathbf{U}(\mathbf{e q}) \\ \text { H3 } & 1754.31 & 4057.02 & 251.64 & 16 \\ \text { H5 } & 2730.12 & 10324.19 & 1788.92 & 13 \\ \text { H15 } & 1144.25 & 2983.65 & 2660.92 & 11 \\ \text { H13 } & 2475.89 & 4626.4 & 6376.92 & 13 \\ & & \text { S27 } & & \end{array}$




$\begin{array}{ccccc}\text { H1 } & 7249 & 8566.77 & 2330.31 & 16 \\ \text { H3A } & 7574.37 & 12307.04 & 1126.89 & 17 \\ \text { H4 } & 4465.14 & 12259.22 & 1203.19 & 15 \\ \text { H2 } & 8964.96 & 10478.41 & 1705.75 & 18 \\ \text { H12 } & 3643.69 & 6941.05 & 6088.04 & 12\end{array}$




\section{4. ${ }^{1} \mathrm{H},{ }^{13} \mathrm{C}$, and ${ }^{19} \mathrm{~F}$ NMR spectra}

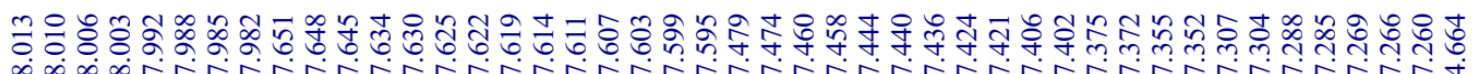

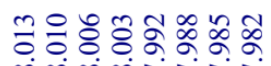

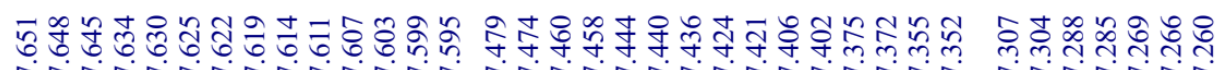

oinoioinitit

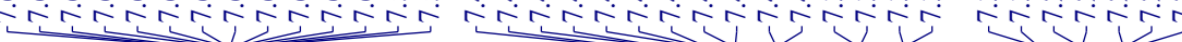
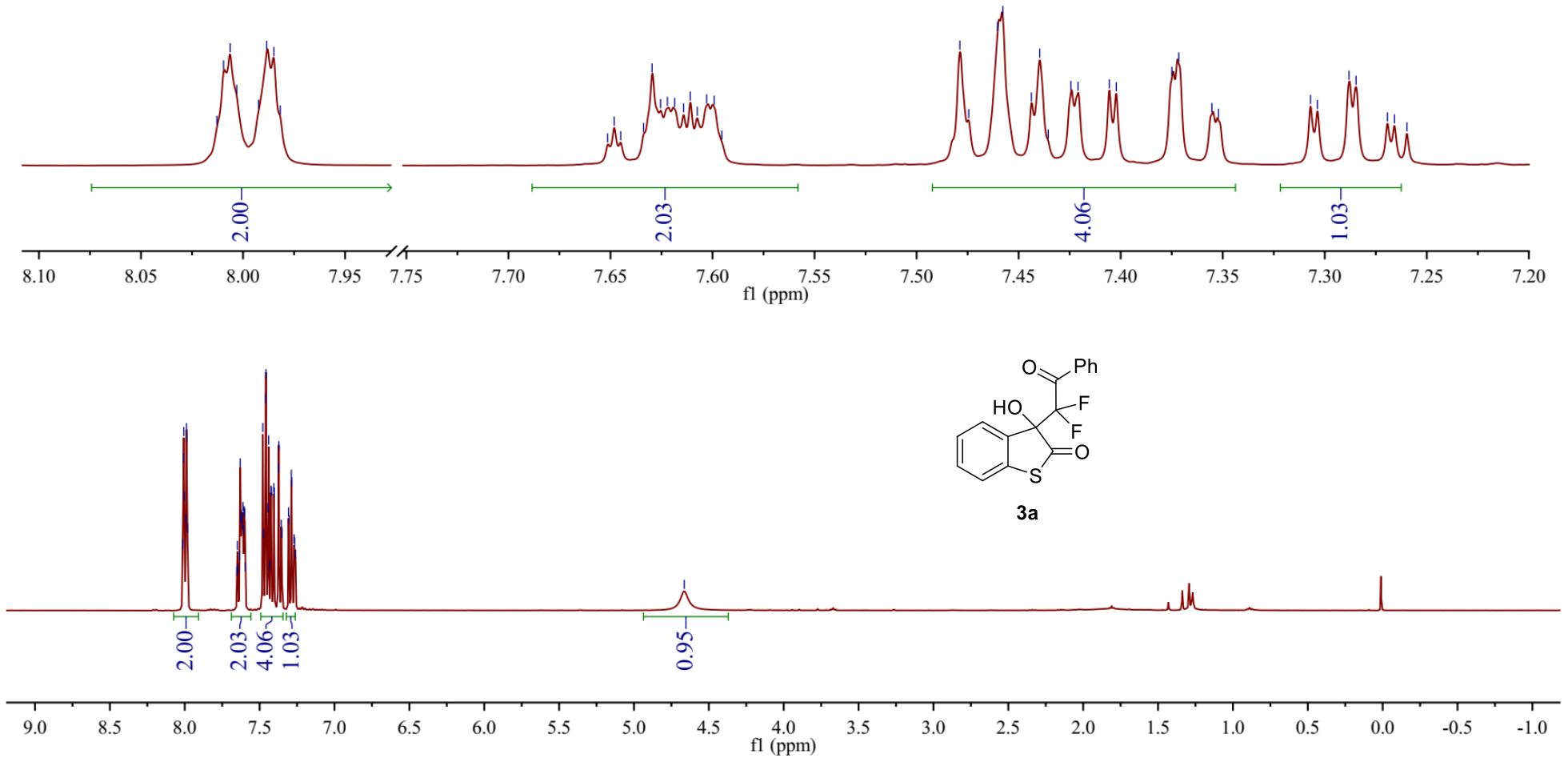

${ }^{1} \mathrm{H}$ NMR spectra of $\mathbf{3 a}$ in $\mathrm{CDCl}_{3}(400 \mathrm{MHz})$ 

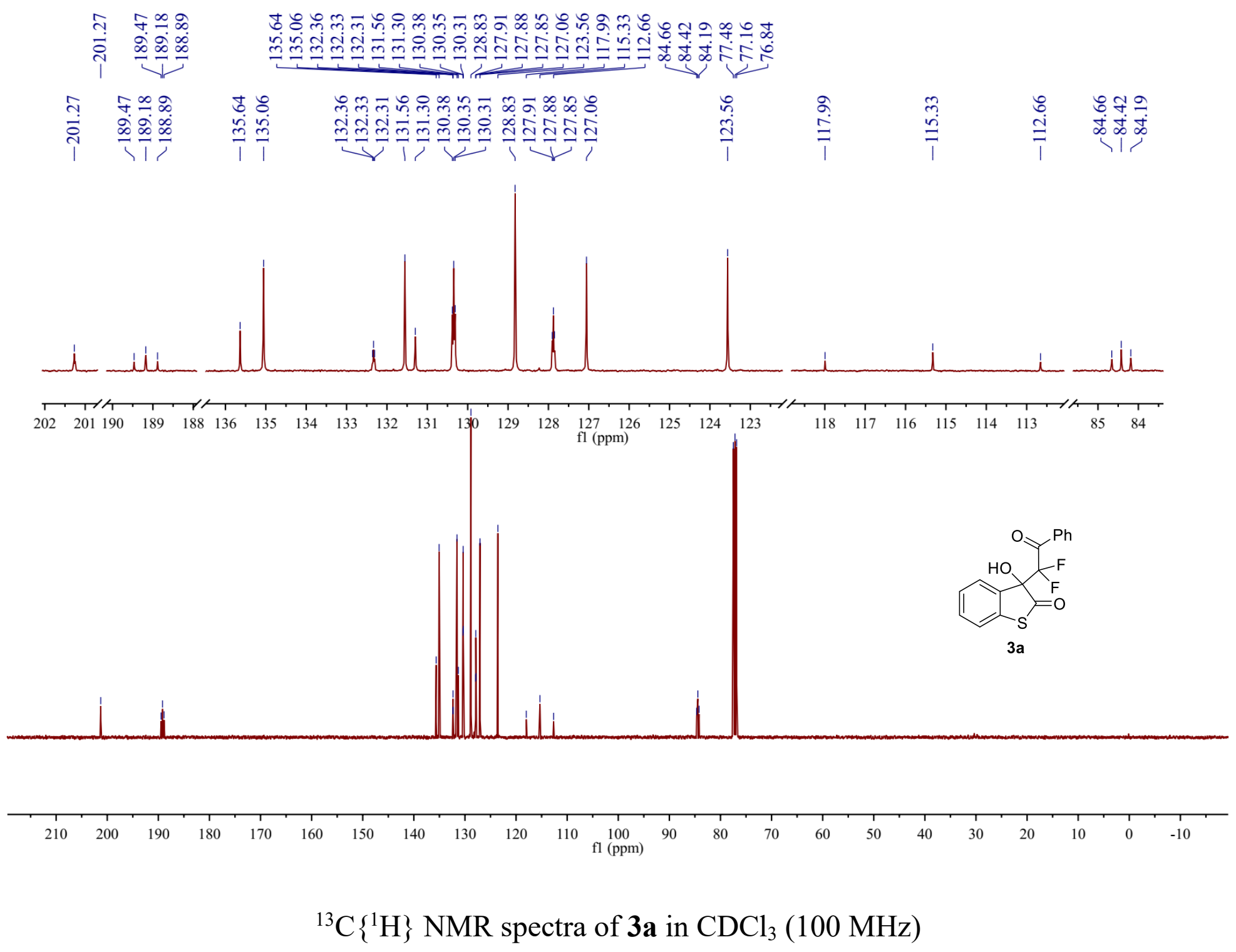


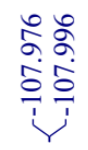

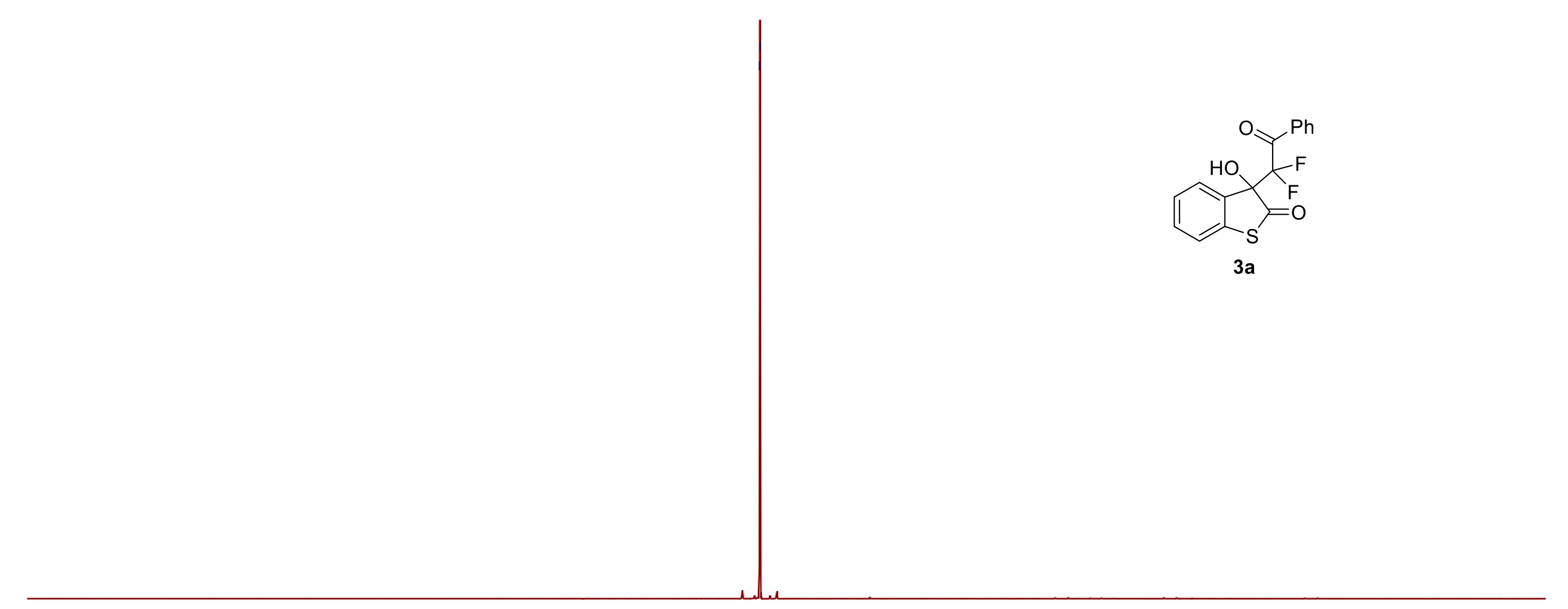

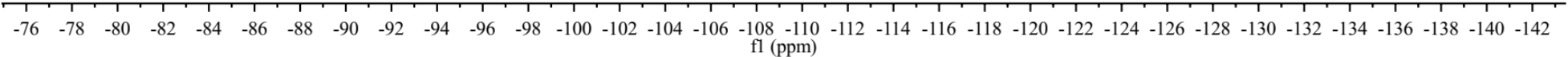

${ }^{19} \mathrm{~F}$ NMR spectra of $\mathbf{3 a}$ in $\mathrm{CDCl}_{3}(376 \mathrm{MHz})$ 

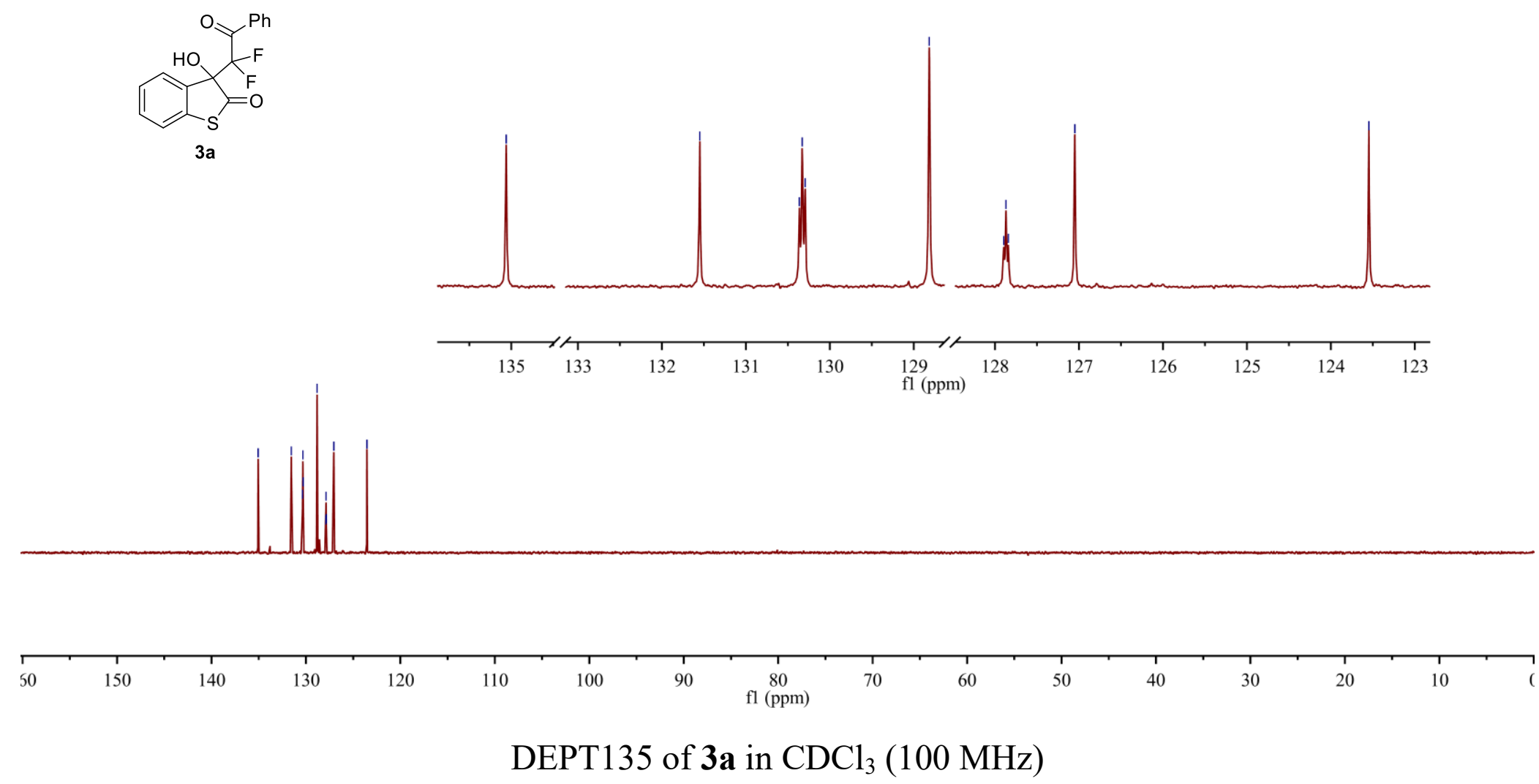


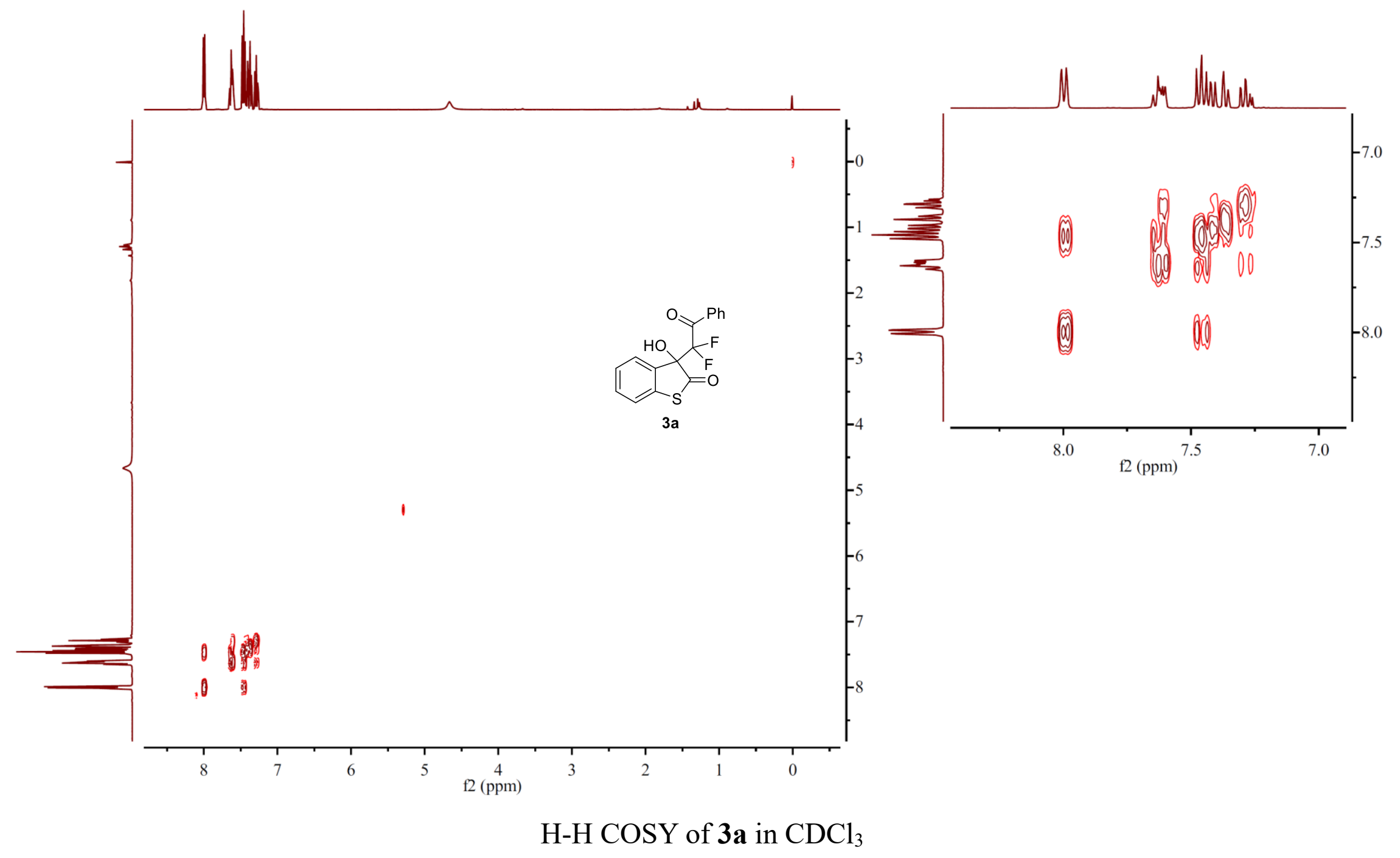




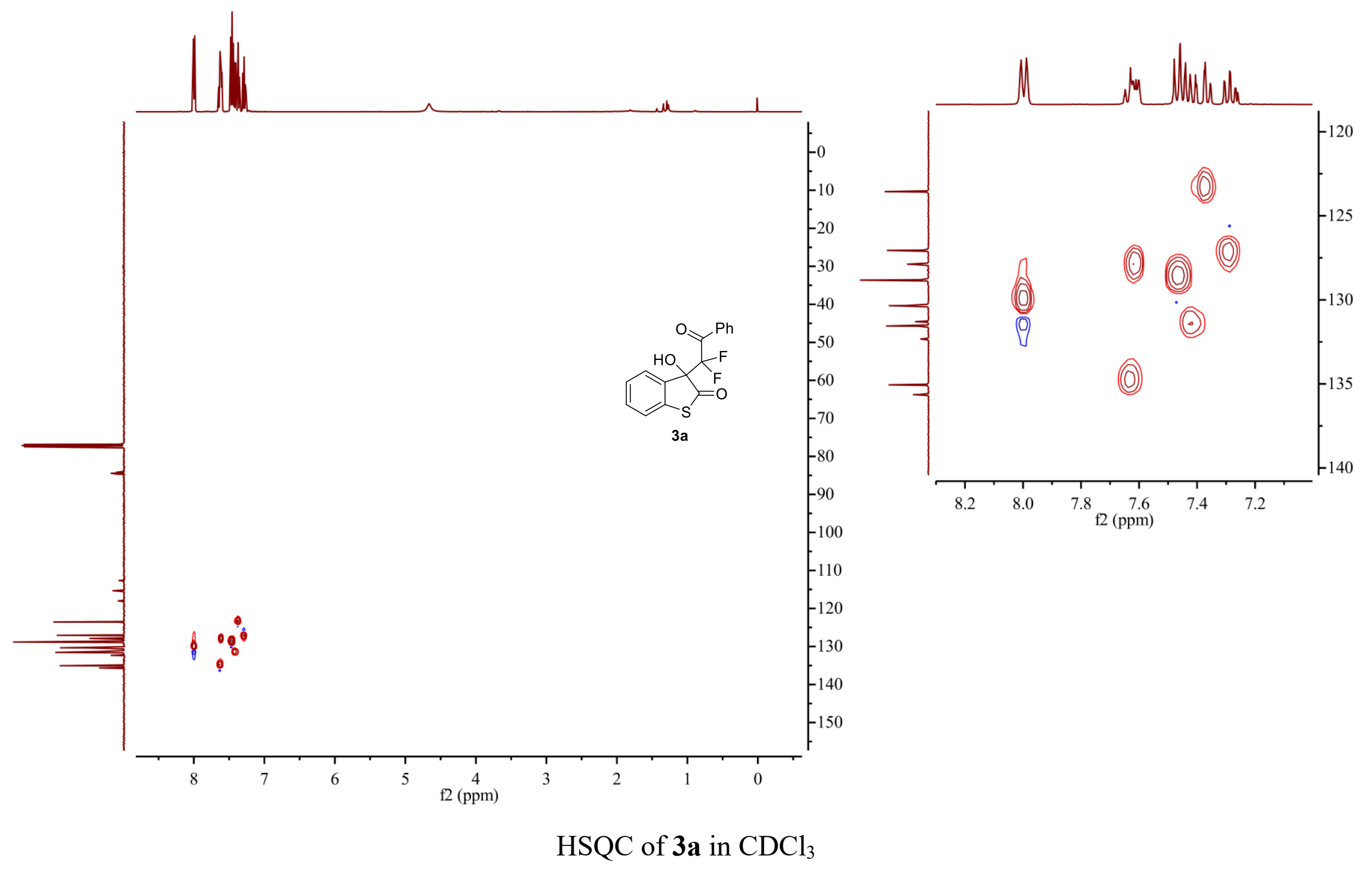




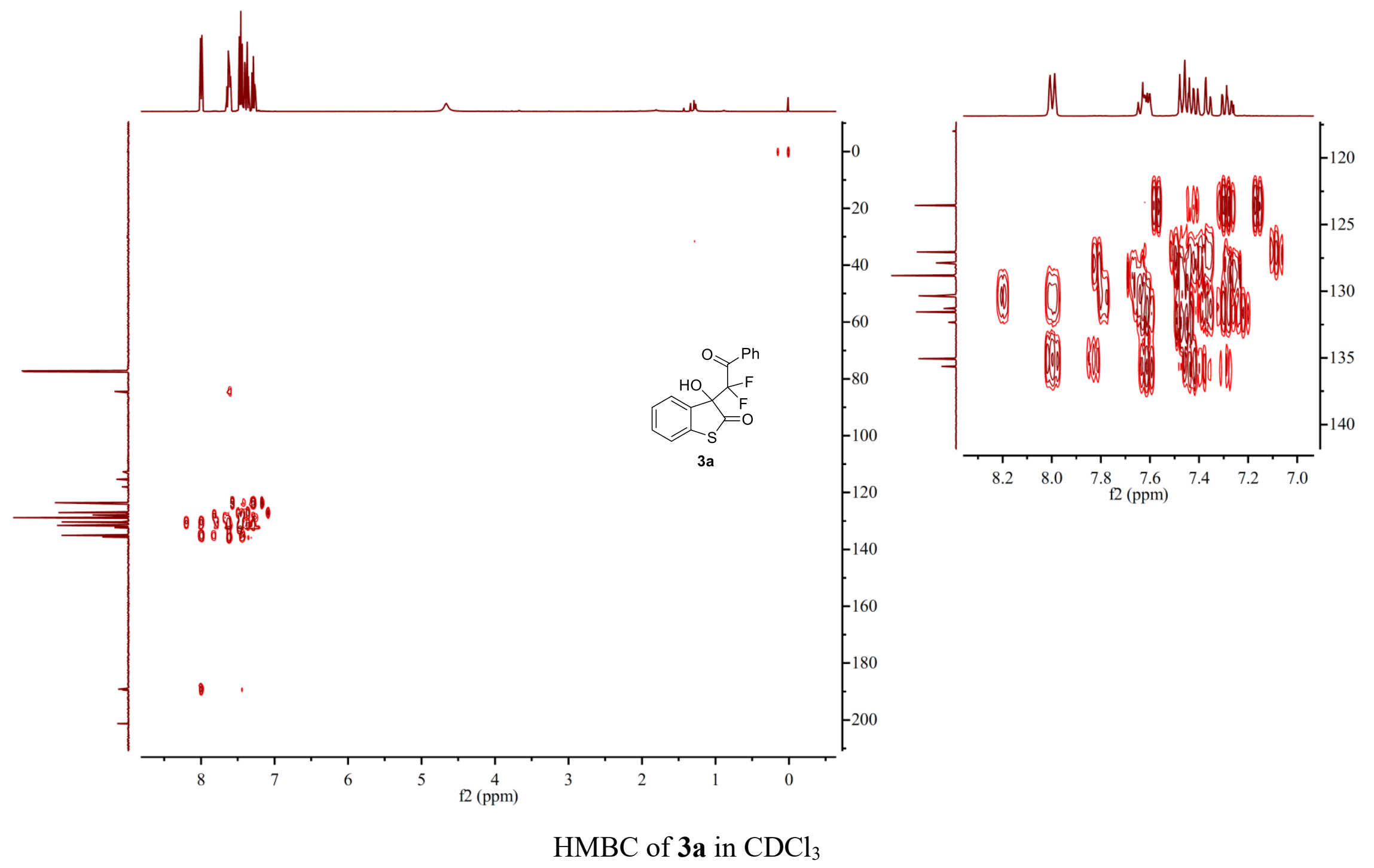




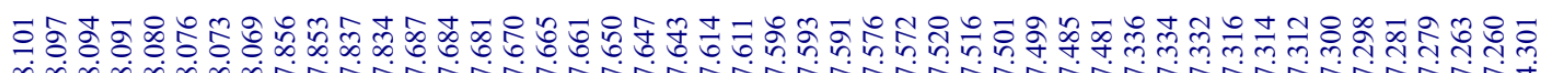

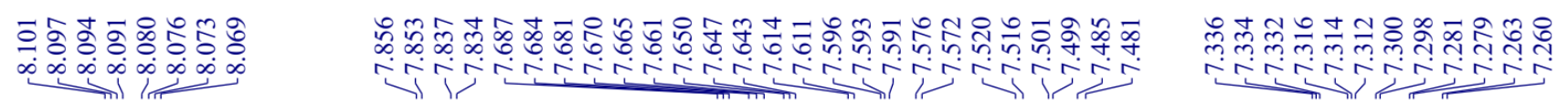

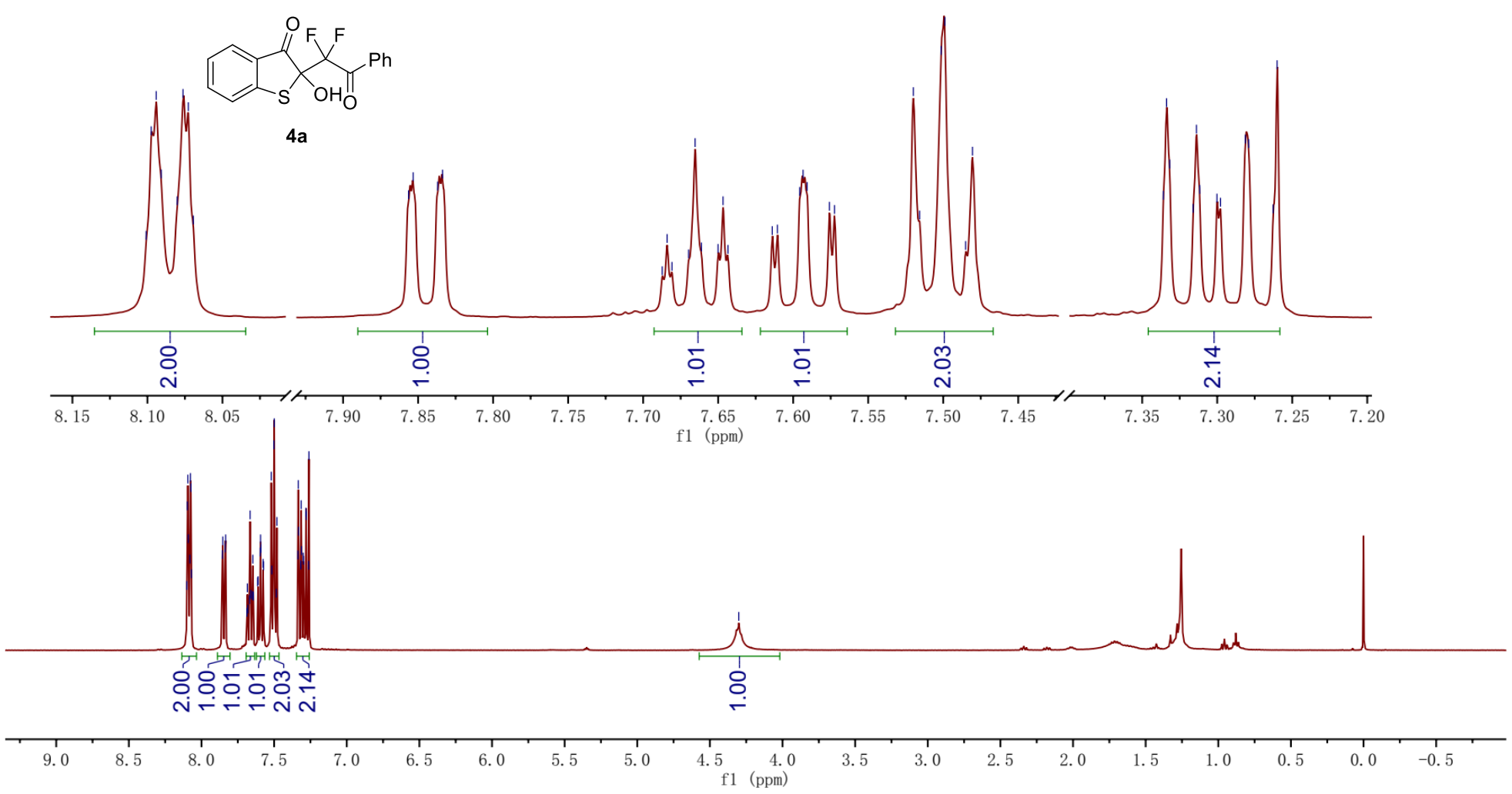

${ }^{1} \mathrm{H}$ NMR spectra of $\mathbf{4 a}$ in $\mathrm{CDCl}_{3}(400 \mathrm{MHz})$ 

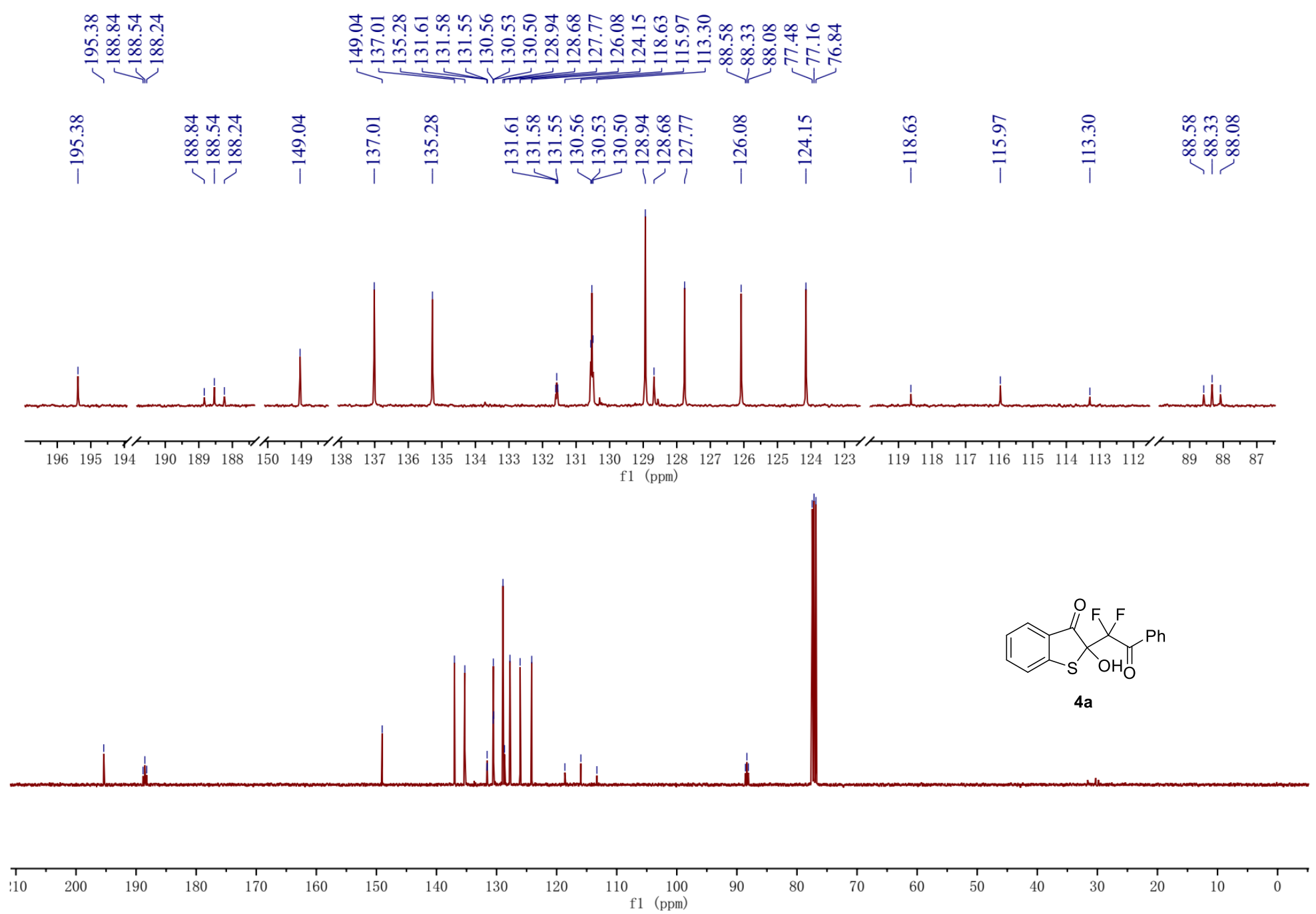

${ }^{13} \mathrm{C}\left\{{ }^{1} \mathrm{H}\right\}$ NMR spectra of $\mathbf{4 a}$ in $\mathrm{CDCl}_{3}(100 \mathrm{MHz})$ 


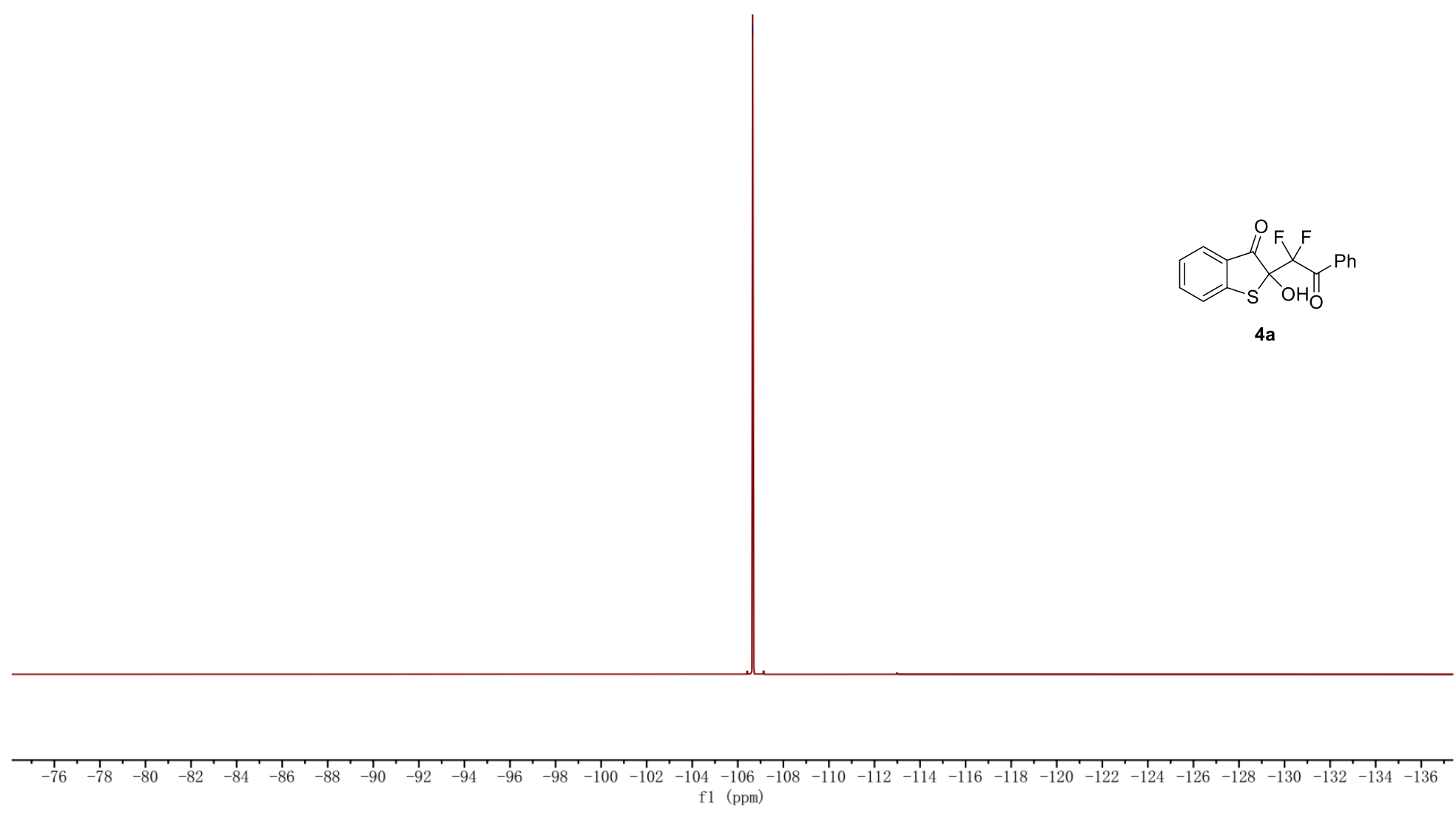

${ }^{19} \mathrm{~F}$ NMR spectra of $\mathbf{4 a}$ in $\mathrm{CDCl}_{3}(376 \mathrm{MHz})$ 


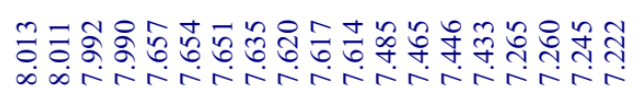

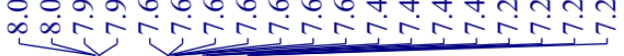

$\stackrel{\substack{n \\ i}}{\stackrel{1}{i}}$

$\underset{\mathrm{f}}{\mathrm{i}}$

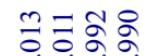

我藏

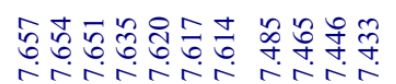

武证证?

คำํำ

राग्र
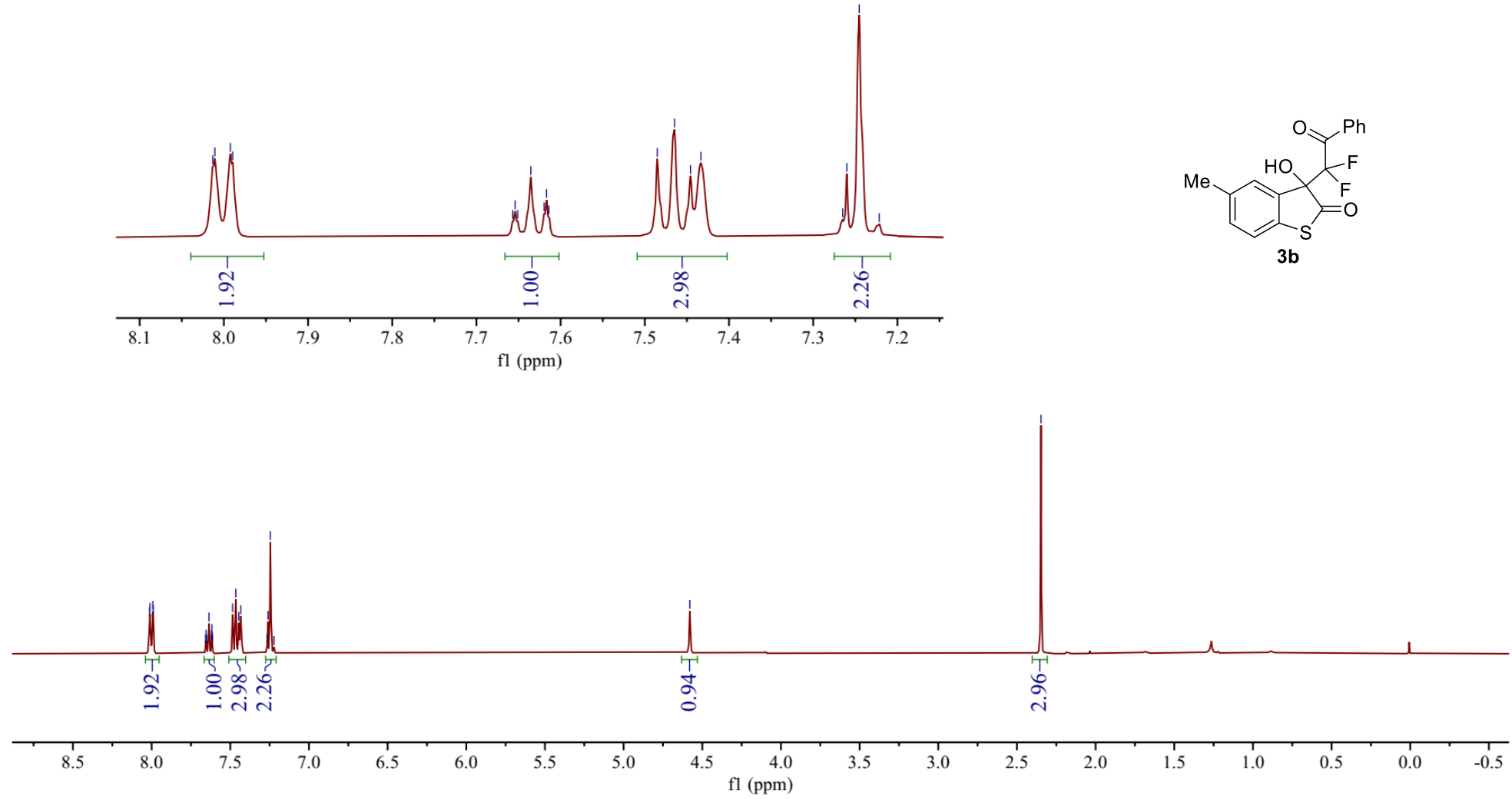

${ }^{1} \mathrm{H}$ NMR spectra of $\mathbf{3 b}$ in $\mathrm{CDCl}_{3}(400 \mathrm{MHz})$ 


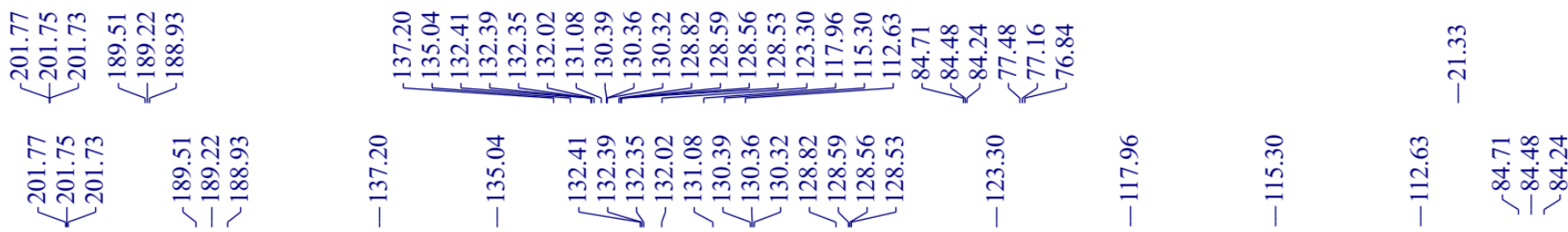
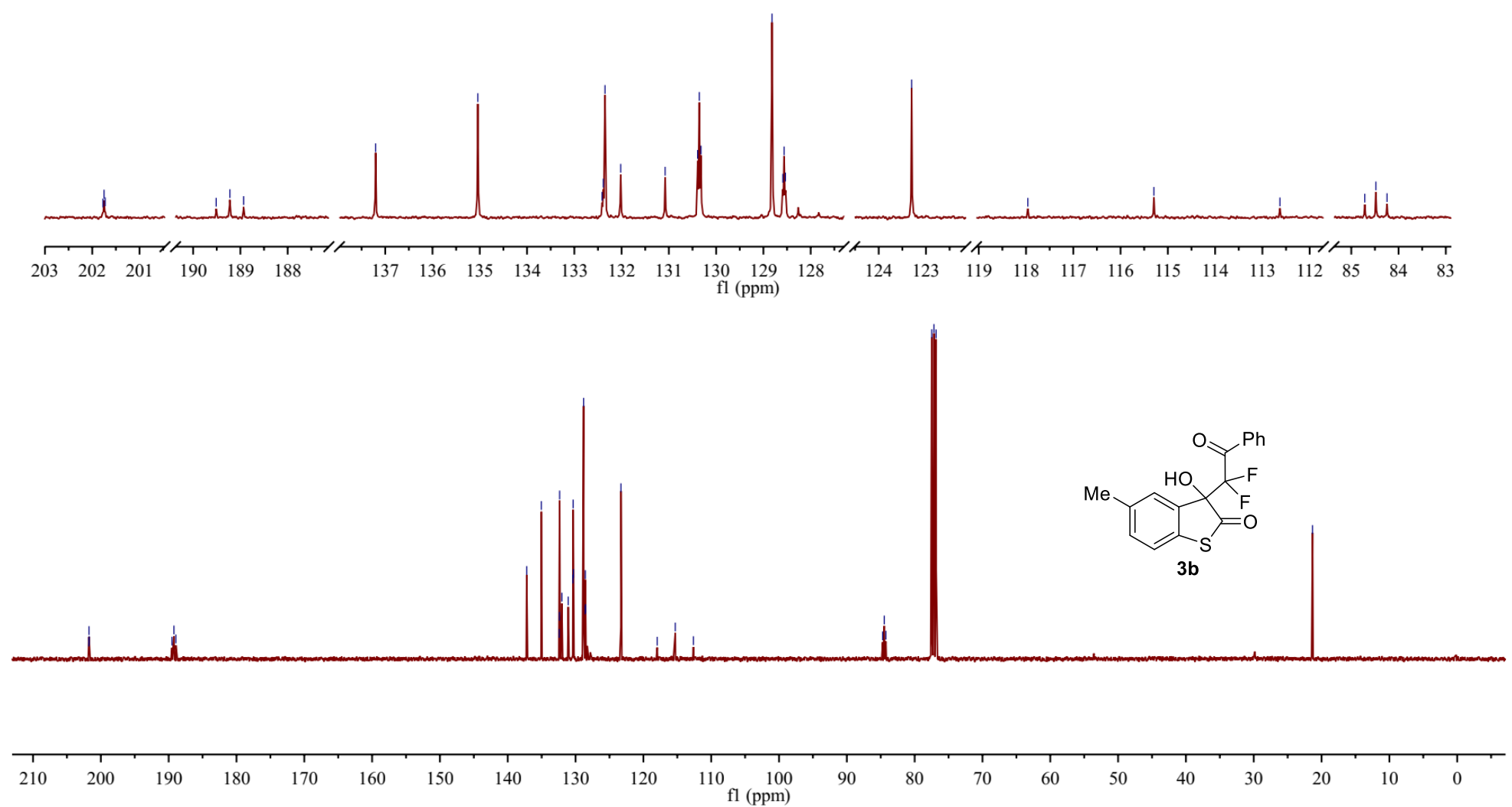

${ }^{13} \mathrm{C}\left\{{ }^{1} \mathrm{H}\right\}$ NMR spectra of $\mathbf{3 b}$ in $\mathrm{CDCl}_{3}(100 \mathrm{MHz})$ 


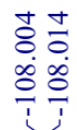

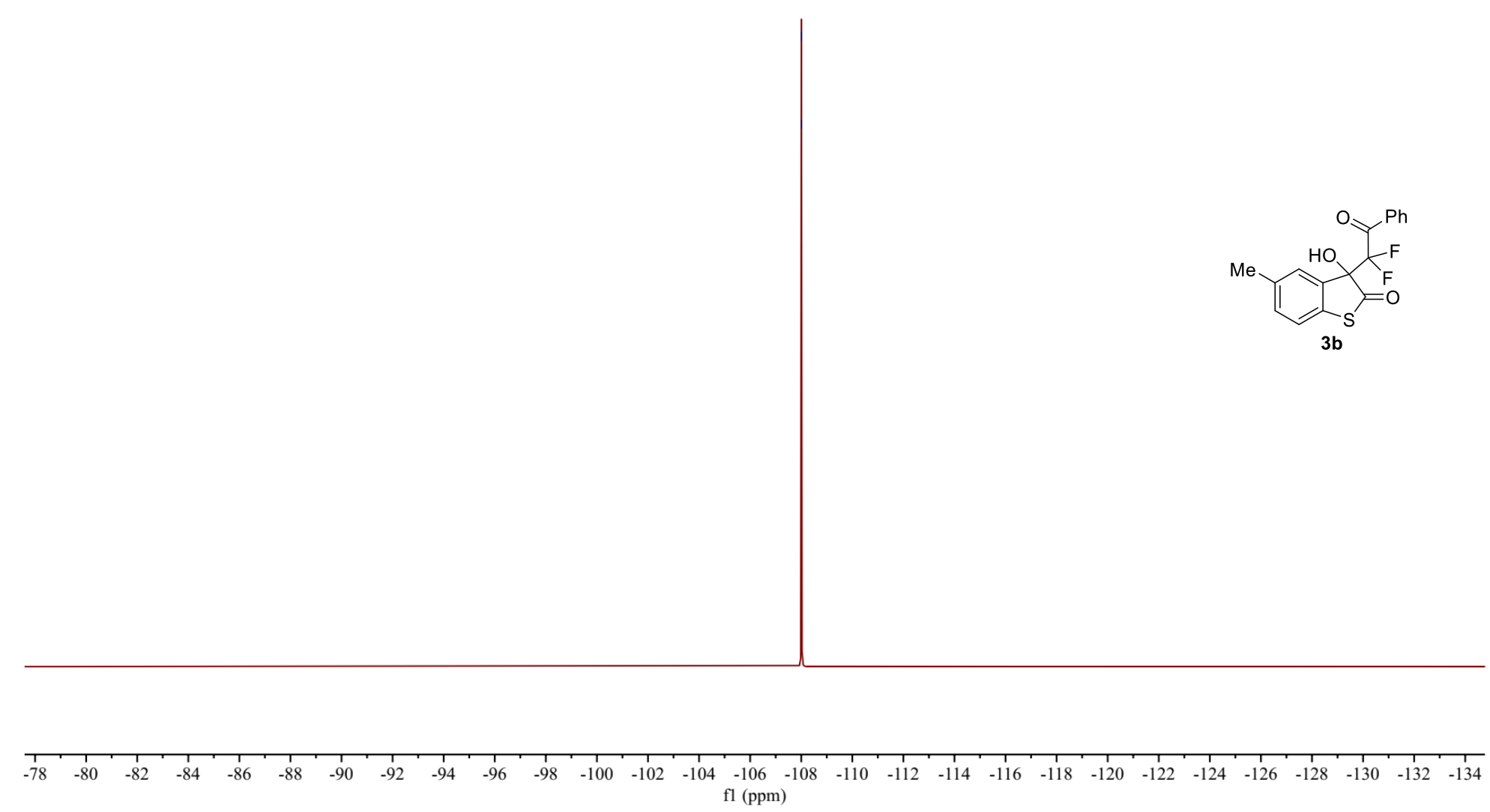

${ }^{19} \mathrm{~F}$ NMR spectra of $\mathbf{3 b}$ in $\mathrm{CDCl}_{3}(376 \mathrm{MHz})$ 


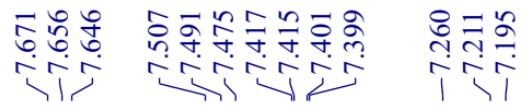
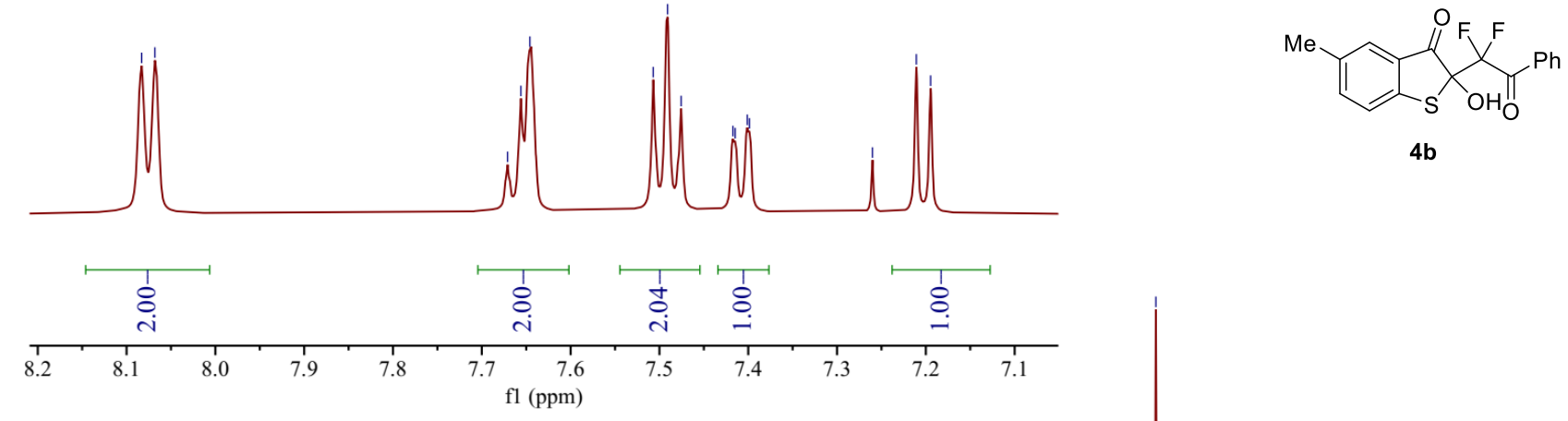

4b

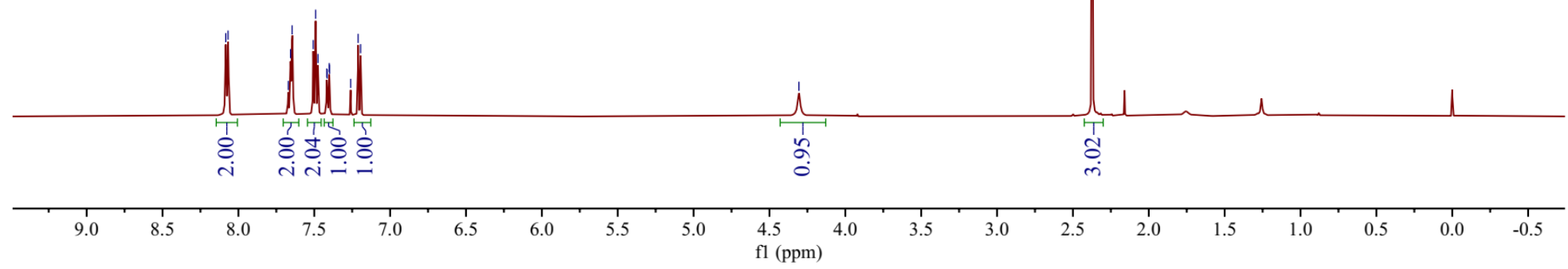

${ }^{1} \mathrm{H}$ NMR spectra of $\mathbf{4 b}$ in $\mathrm{CDCl}_{3}(500 \mathrm{MHz})$ 


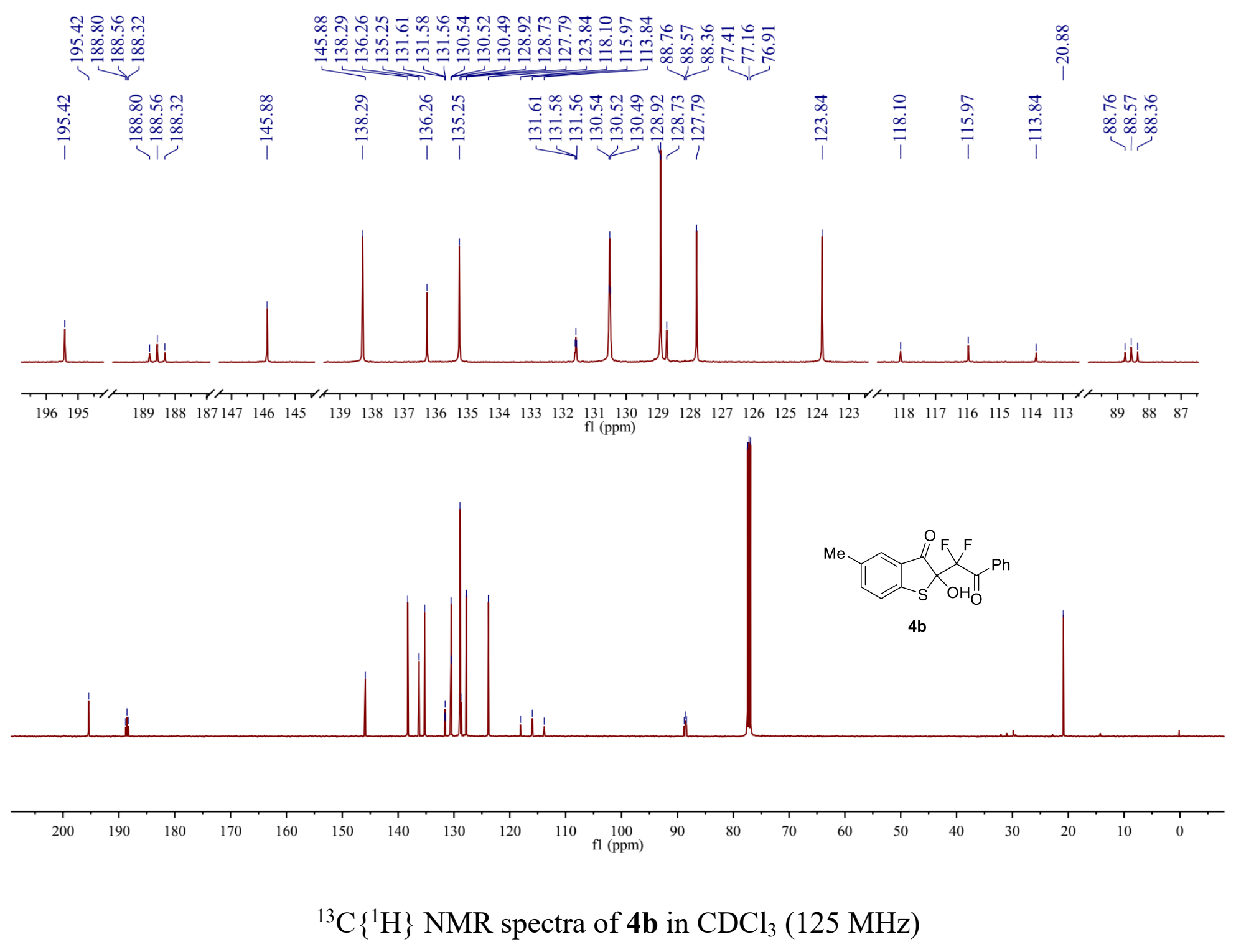




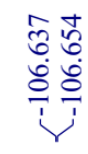

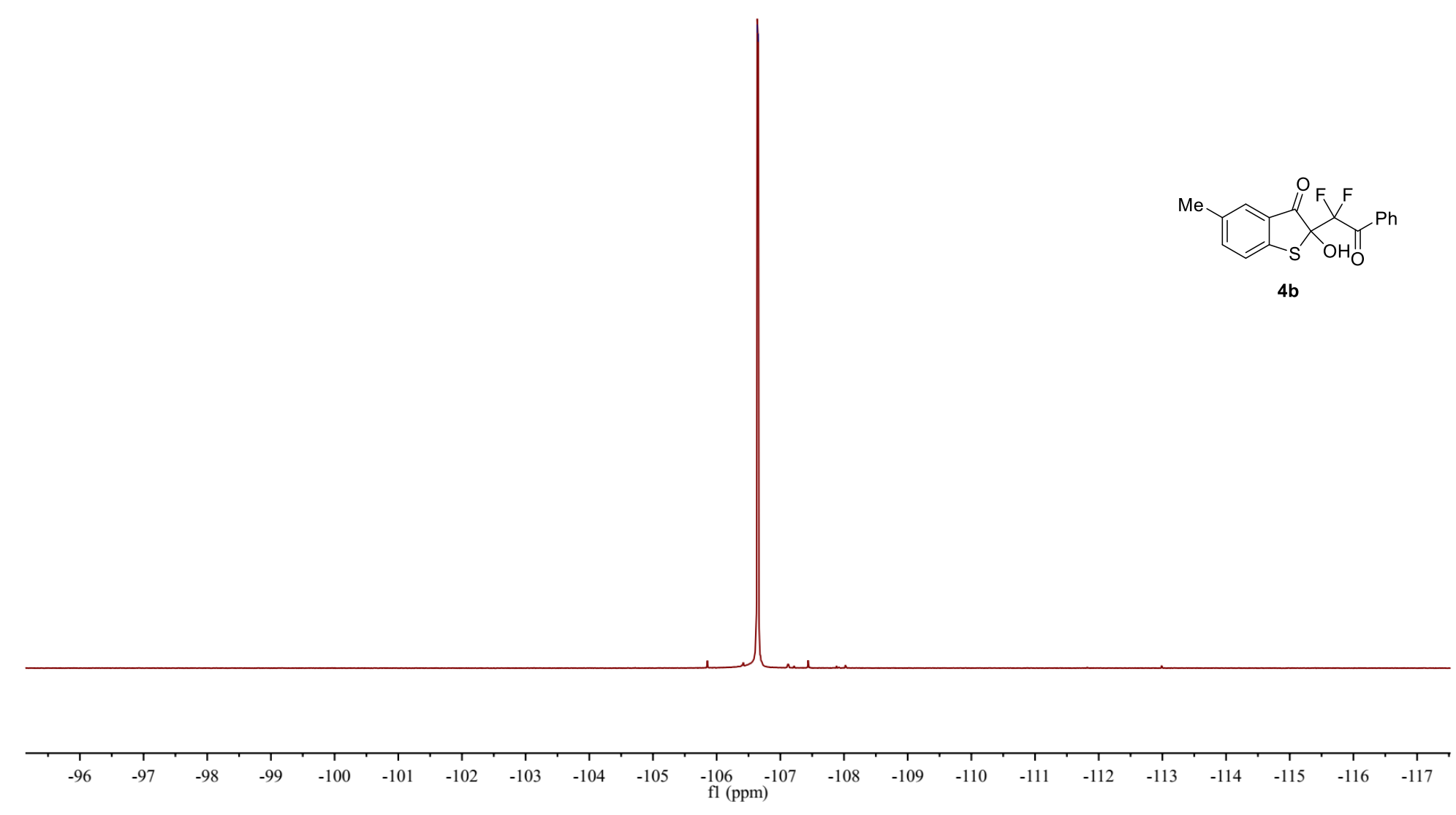

${ }^{19} \mathrm{~F}$ NMR spectra of $\mathbf{4 b}$ in $\mathrm{CDCl}_{3}(376 \mathrm{MHz})$ 


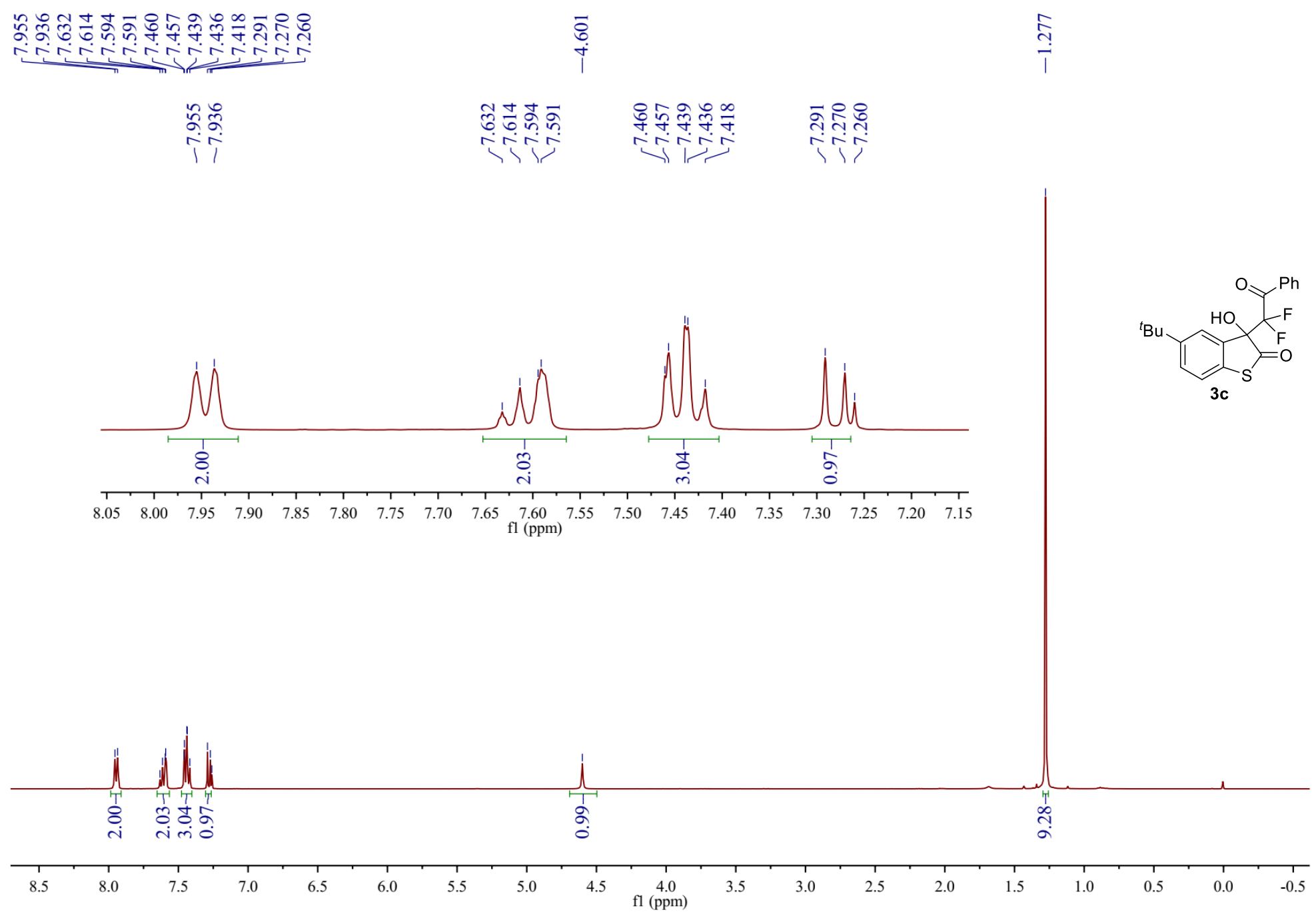

${ }^{1} \mathrm{H}$ NMR spectra of $\mathbf{3 c}$ in $\mathrm{CDCl}_{3}(400 \mathrm{MHz})$ 

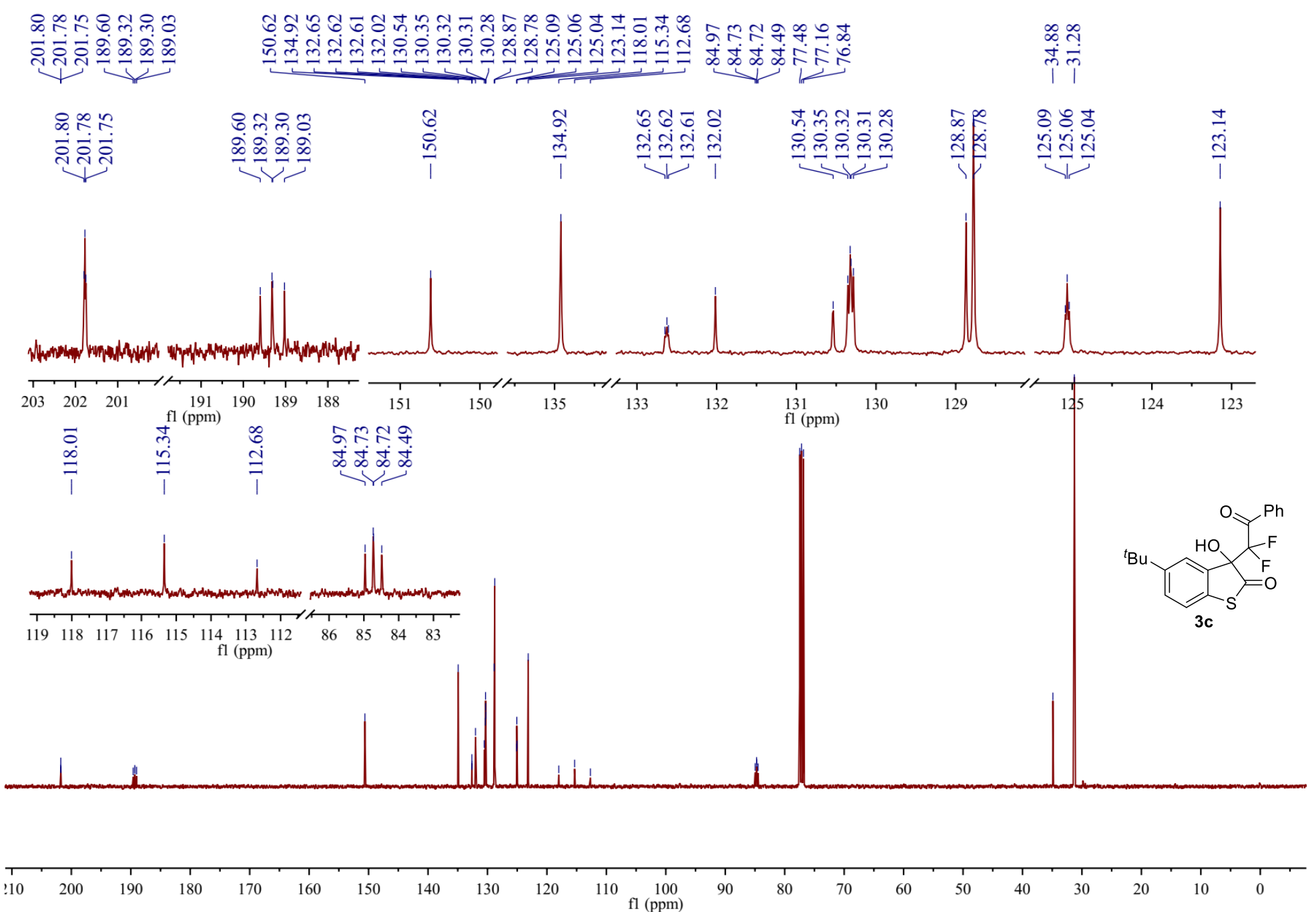

${ }^{13} \mathrm{C}\left\{{ }^{1} \mathrm{H}\right\}$ NMR spectra of $\mathbf{3 c}$ in $\mathrm{CDCl}_{3}(100 \mathrm{MHz})$ 


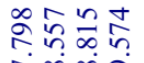

은

i i i

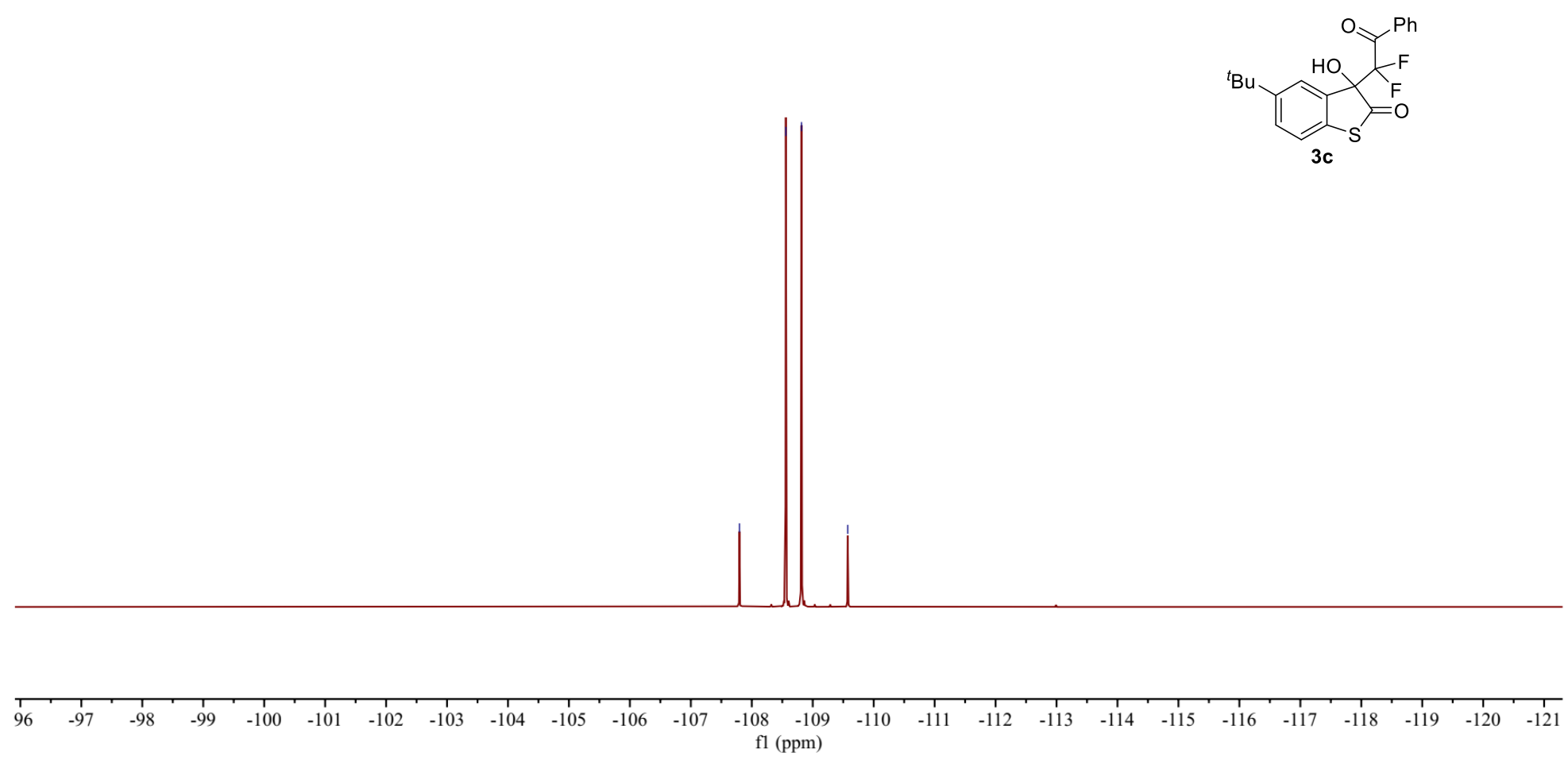

${ }^{19} \mathrm{~F}$ NMR spectra of $\mathbf{3 c}$ in $\mathrm{CDCl}_{3}(376 \mathrm{MHz})$ 


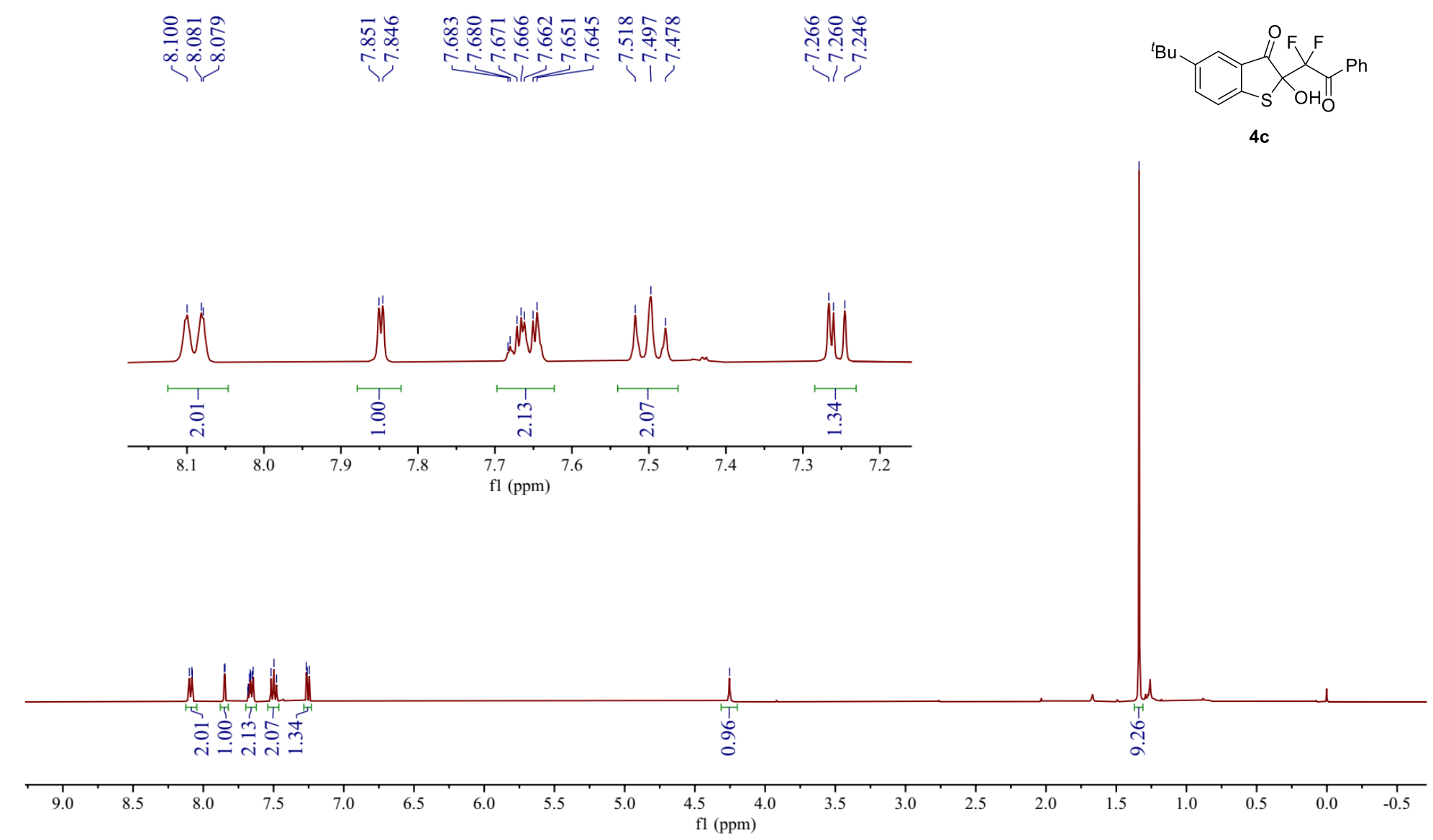

${ }^{1} \mathrm{H}$ NMR spectra of $\mathbf{4 c}$ in $\mathrm{CDCl}_{3}(400 \mathrm{MHz})$ 


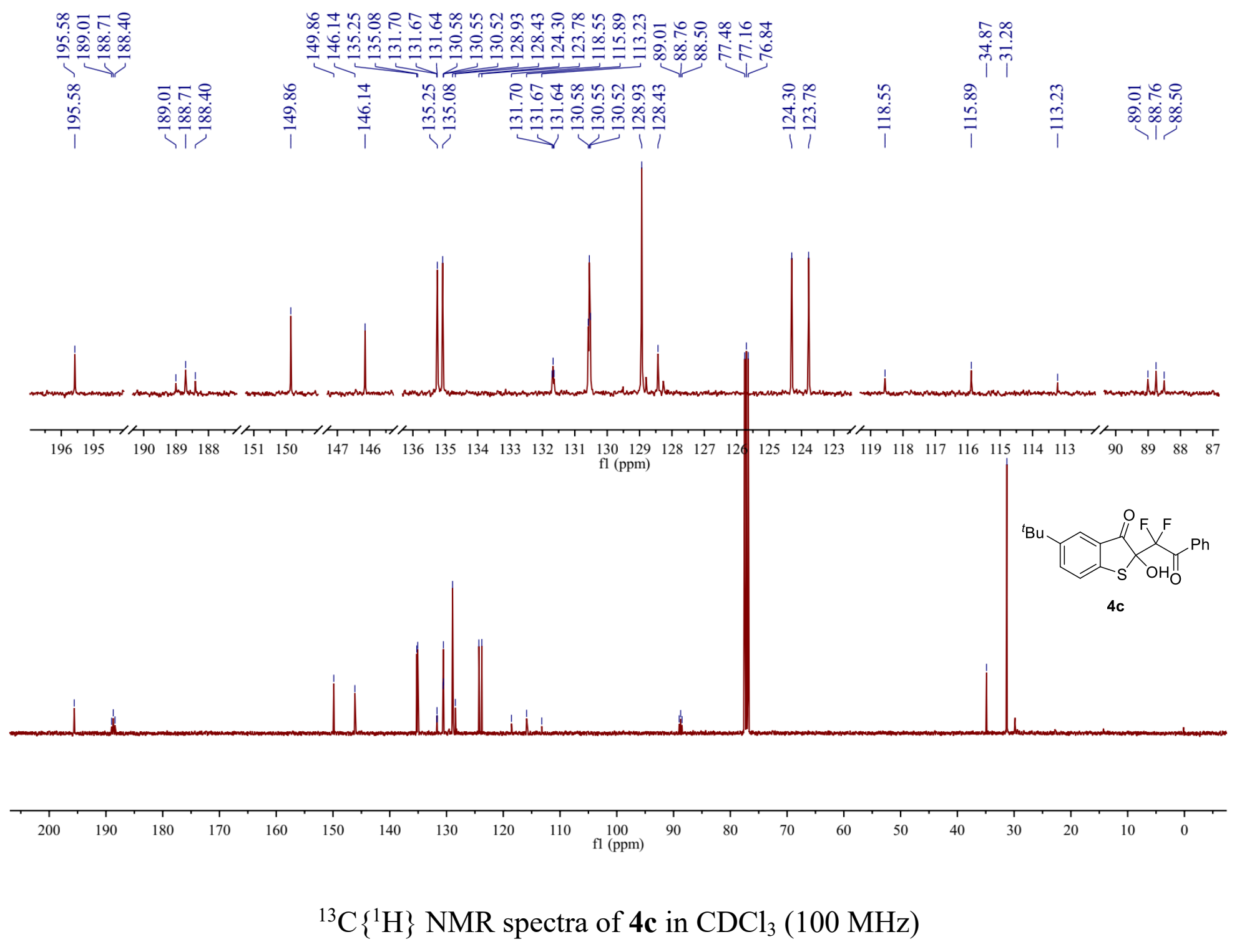




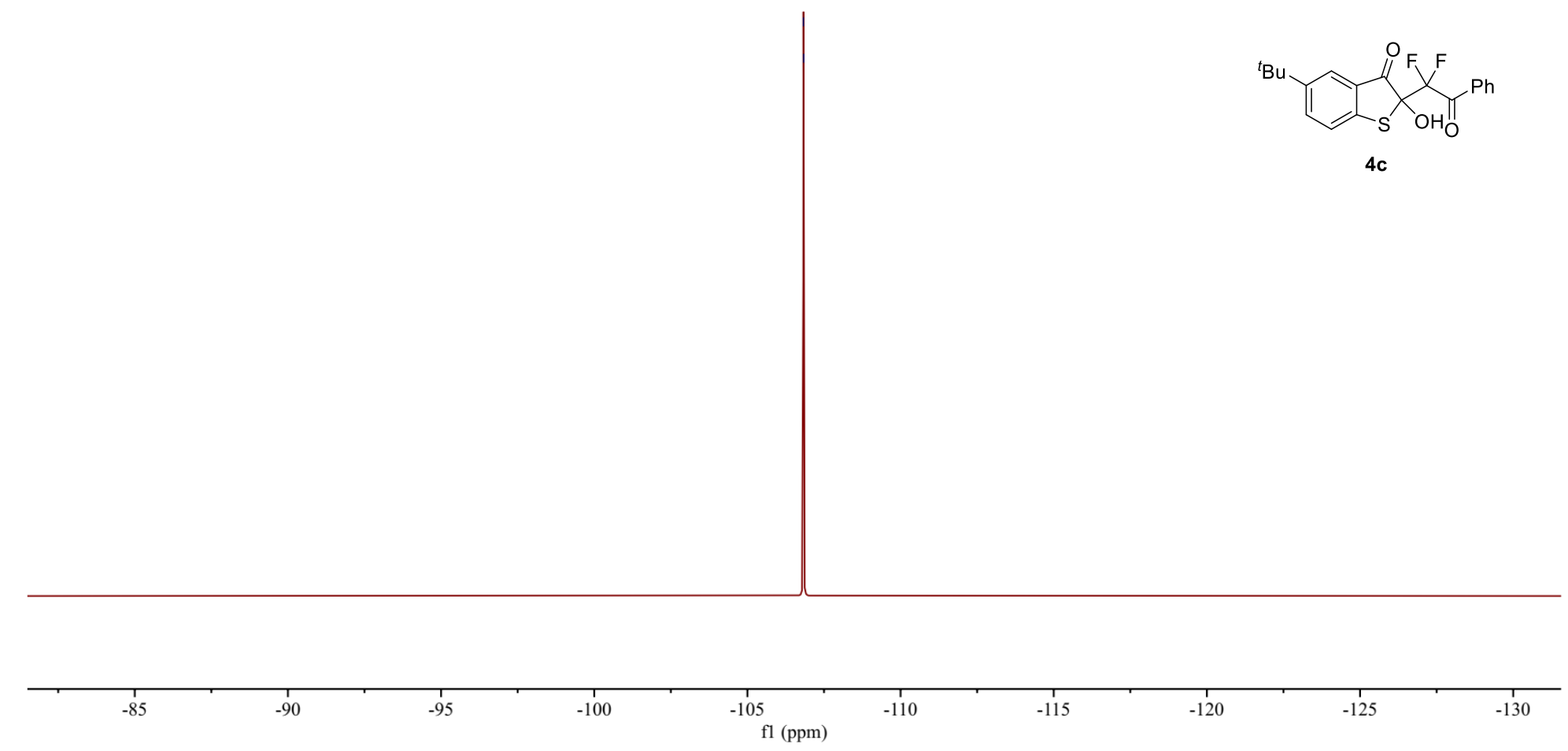

${ }^{19} \mathrm{~F}$ NMR spectra of $\mathbf{4 c}$ in $\mathrm{CDCl}_{3}(376 \mathrm{MHz})$ 
o.

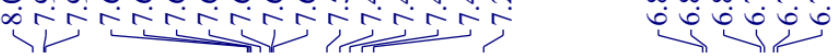
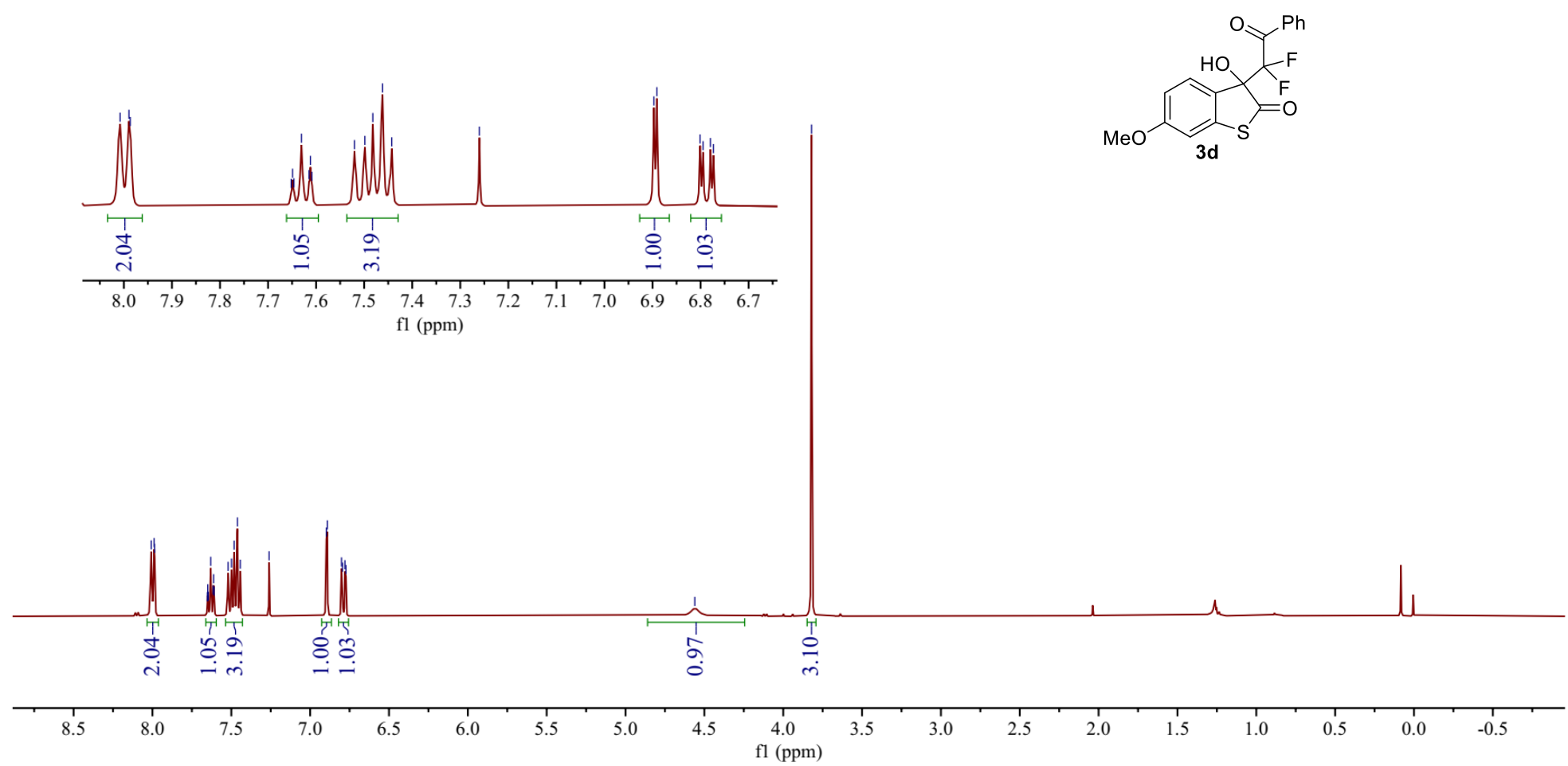

${ }^{1} \mathrm{H}$ NMR spectra of $\mathbf{3 d}$ in $\mathrm{CDCl}_{3}(400 \mathrm{MHz})$ 


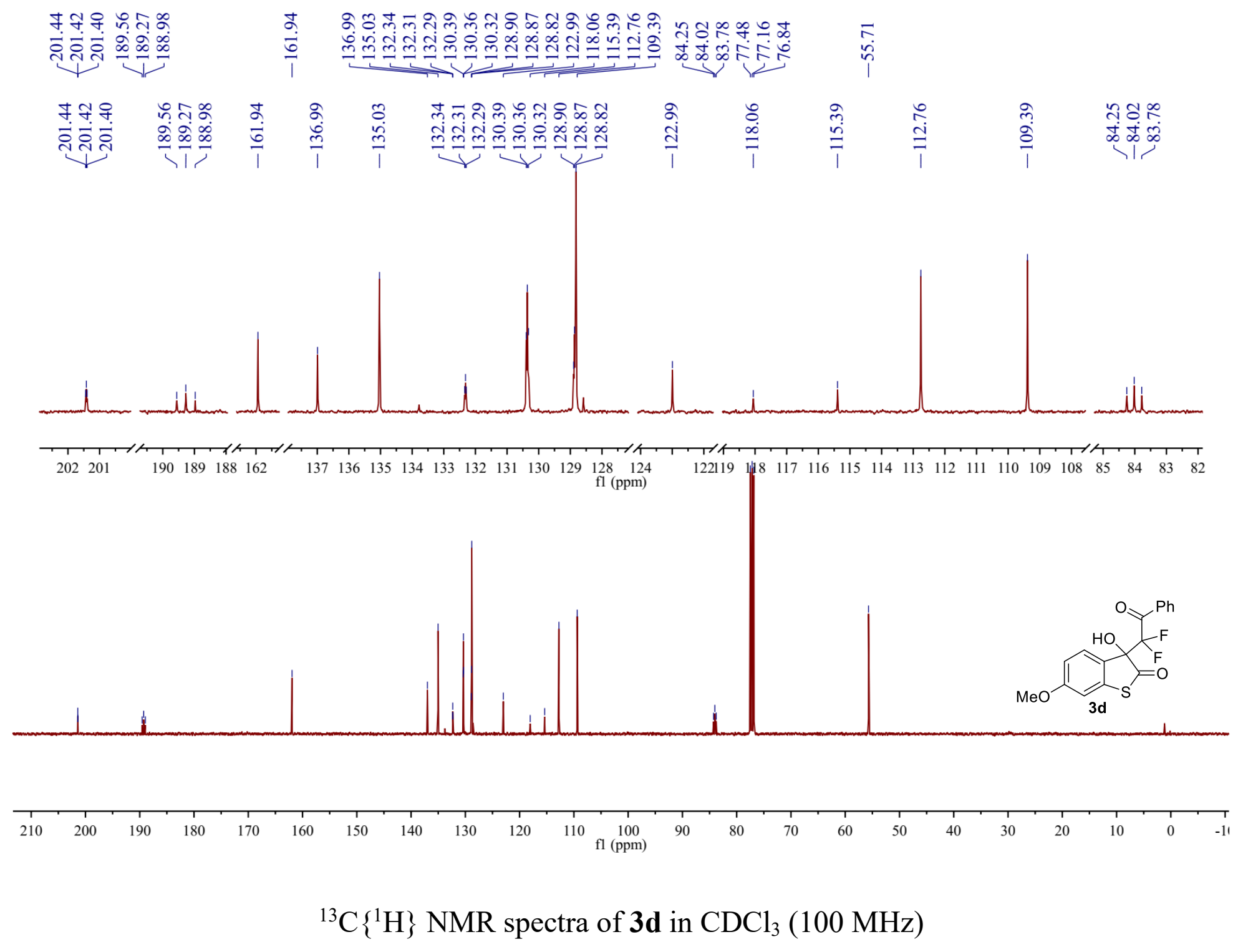




\section{ํㅜ동ㅇㅇㅇ}

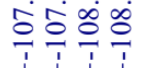

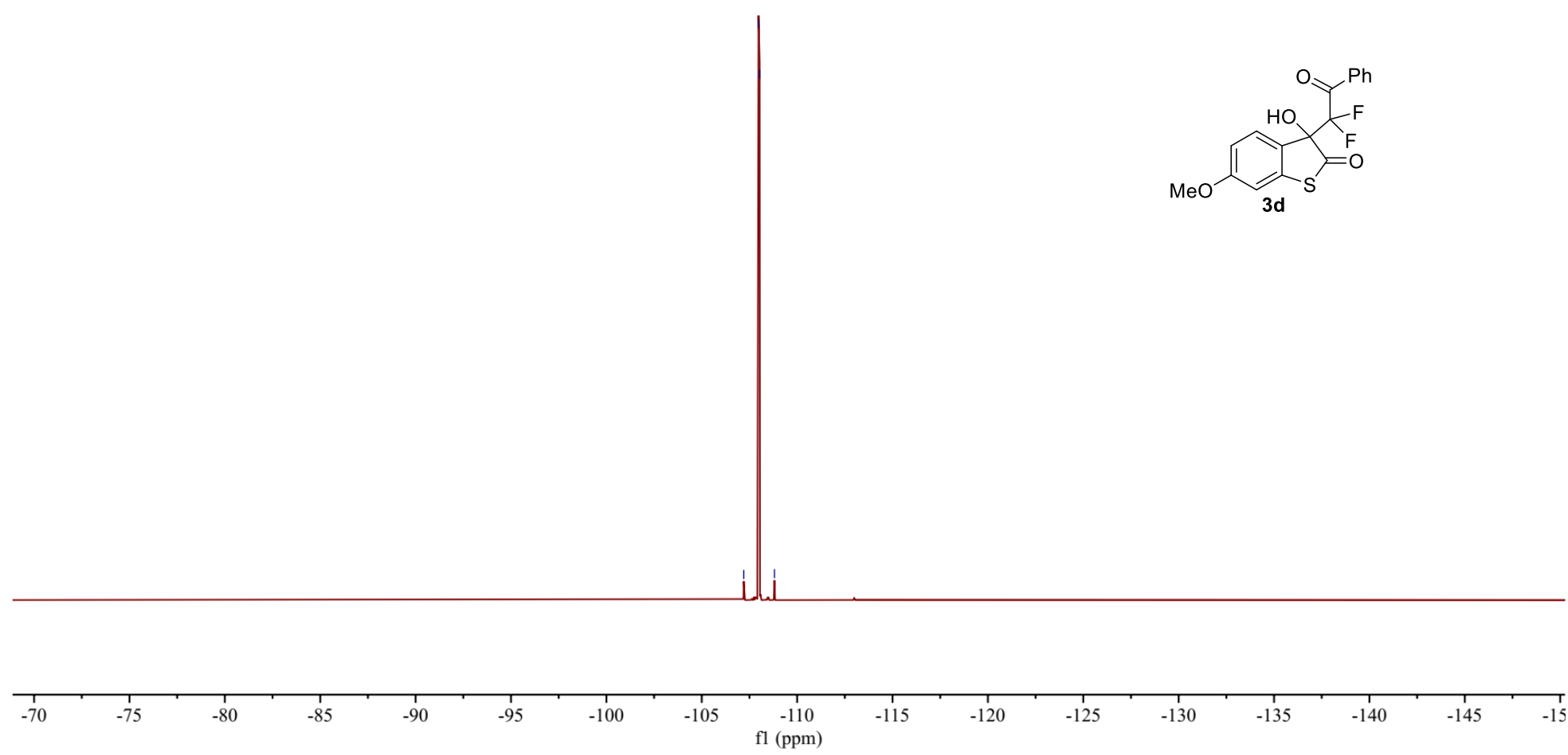

${ }^{19} \mathrm{~F}$ NMR spectra of $\mathbf{3 d}$ in $\mathrm{CDCl}_{3}(376 \mathrm{MHz})$ 


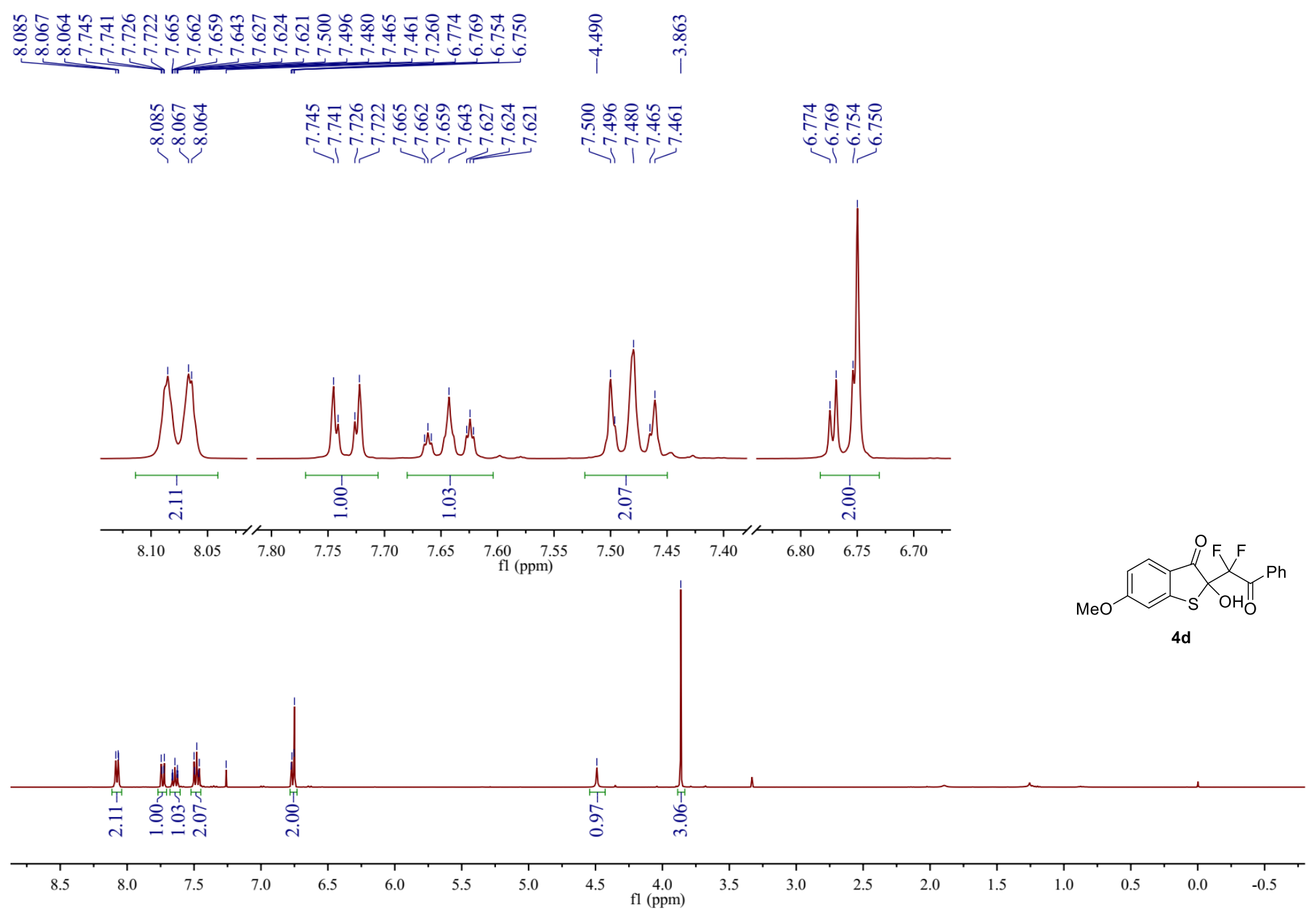

${ }^{1} \mathrm{H}$ NMR spectra of $\mathbf{4 d}$ in $\mathrm{CDCl}_{3}(400 \mathrm{MHz})$ 


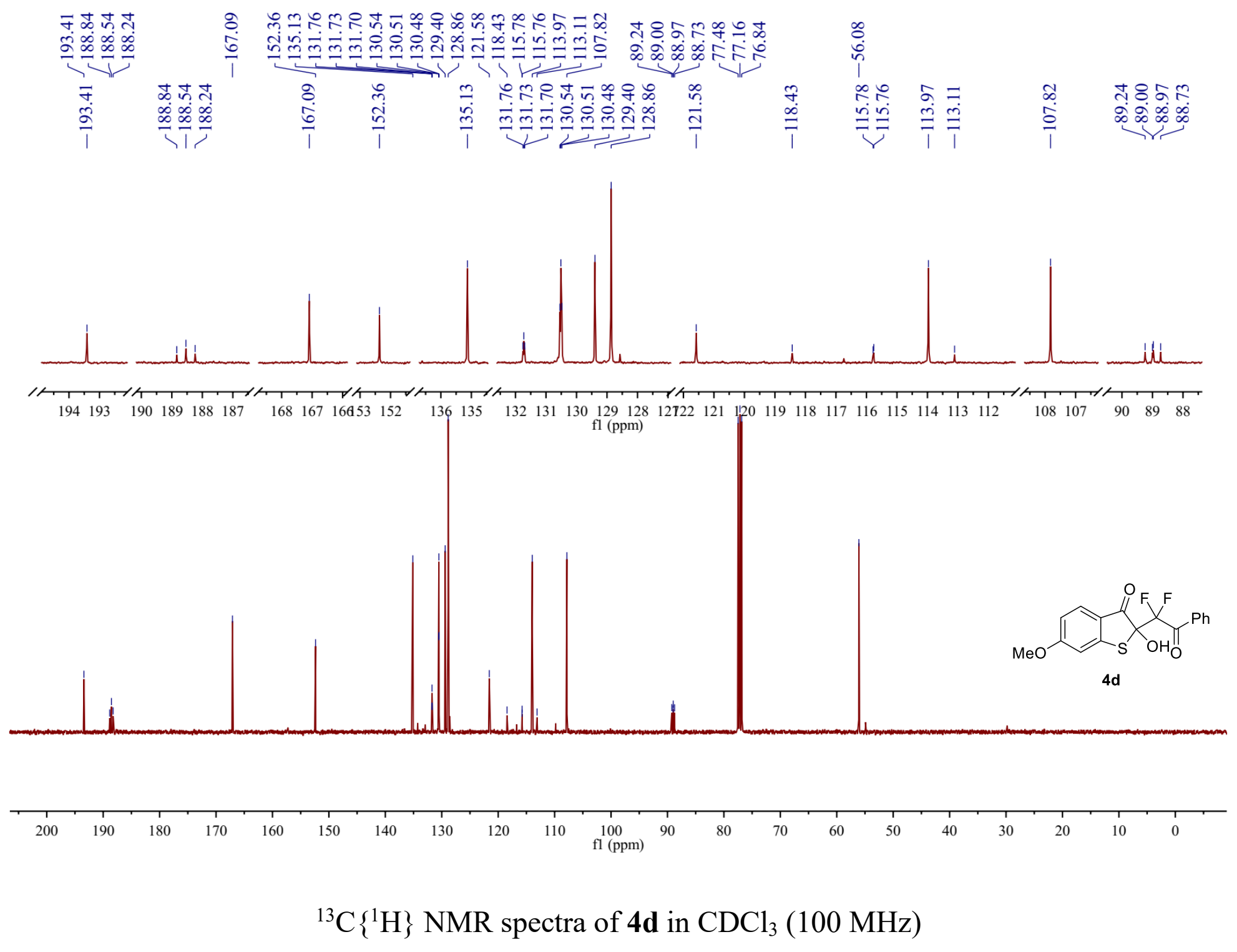




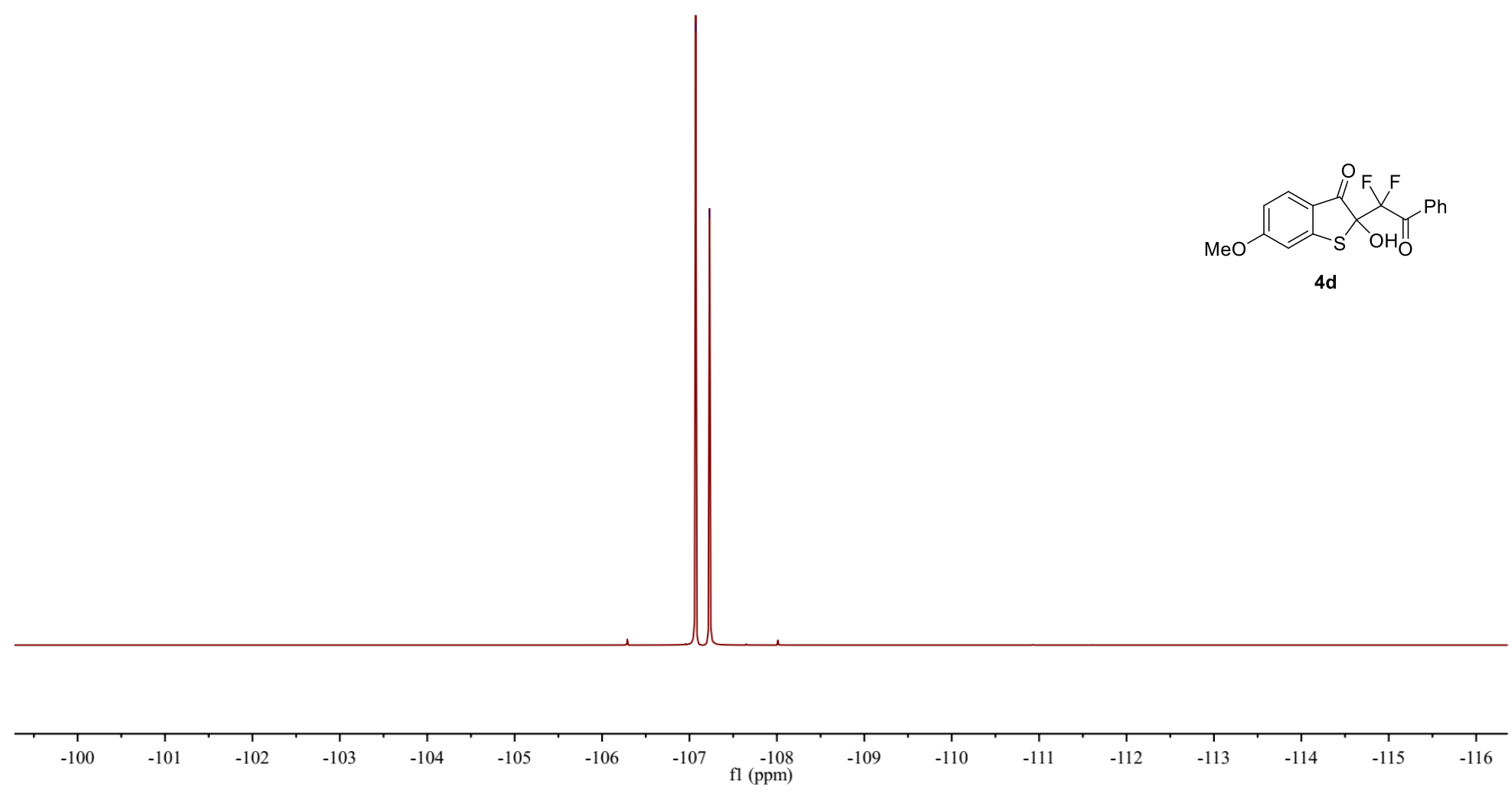

${ }^{19} \mathrm{~F}$ NMR spectra of $\mathbf{4 d}$ in $\mathrm{CDCl}_{3}(376 \mathrm{MHz})$ 


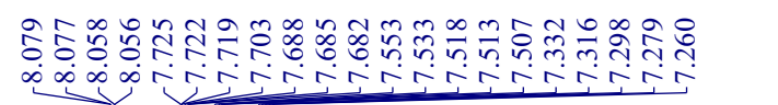

$\underset{\substack{m \\ \stackrel{0}{+}}}{+}$

鼔吕:

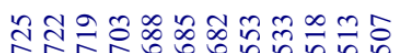

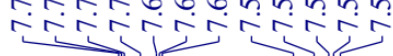

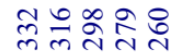

रुगए
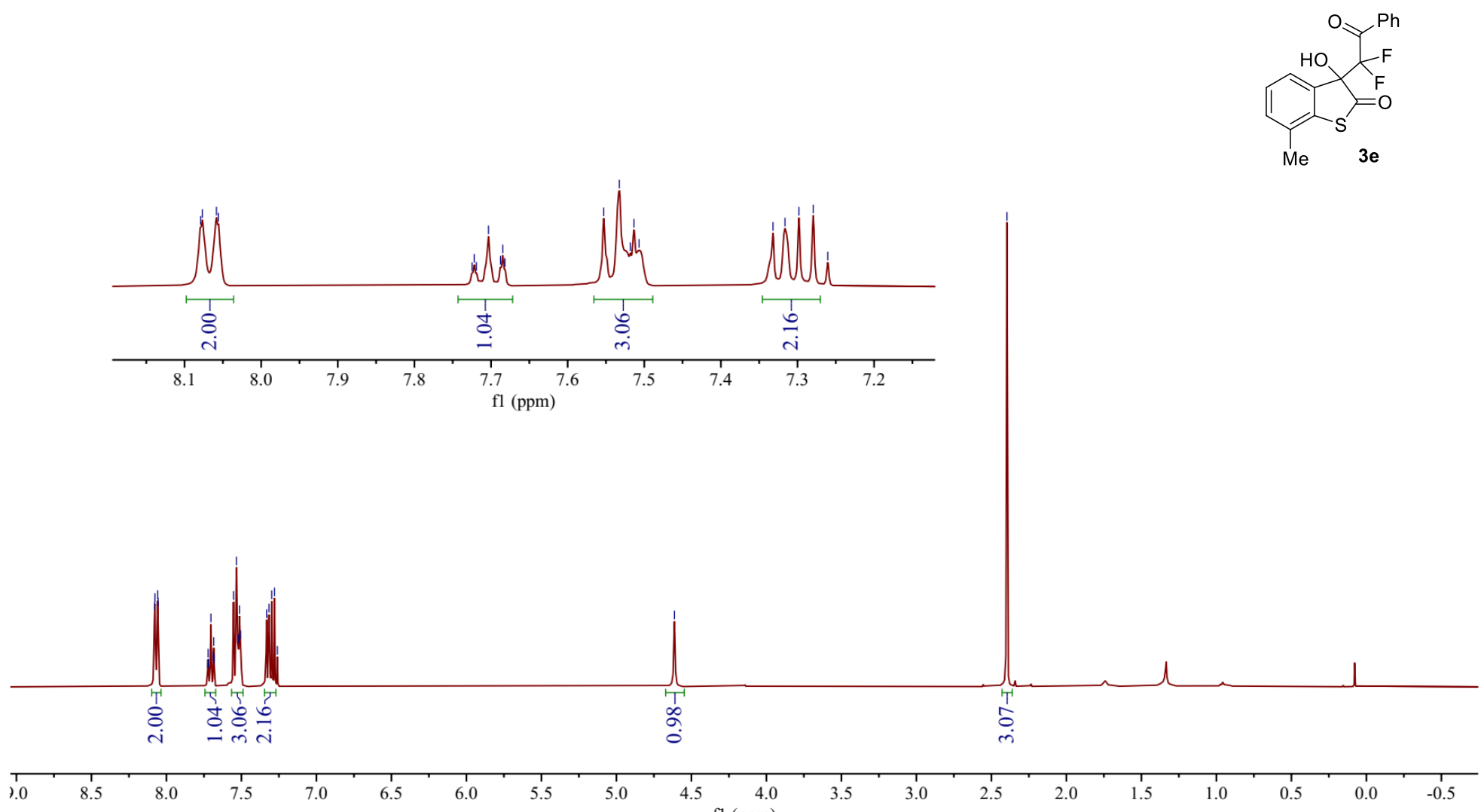

$\mathrm{fl}(\mathrm{ppm})$

${ }^{1} \mathrm{H}$ NMR spectra of $\mathbf{3 e}$ in $\mathrm{CDCl}_{3}(400 \mathrm{MHz})$ 

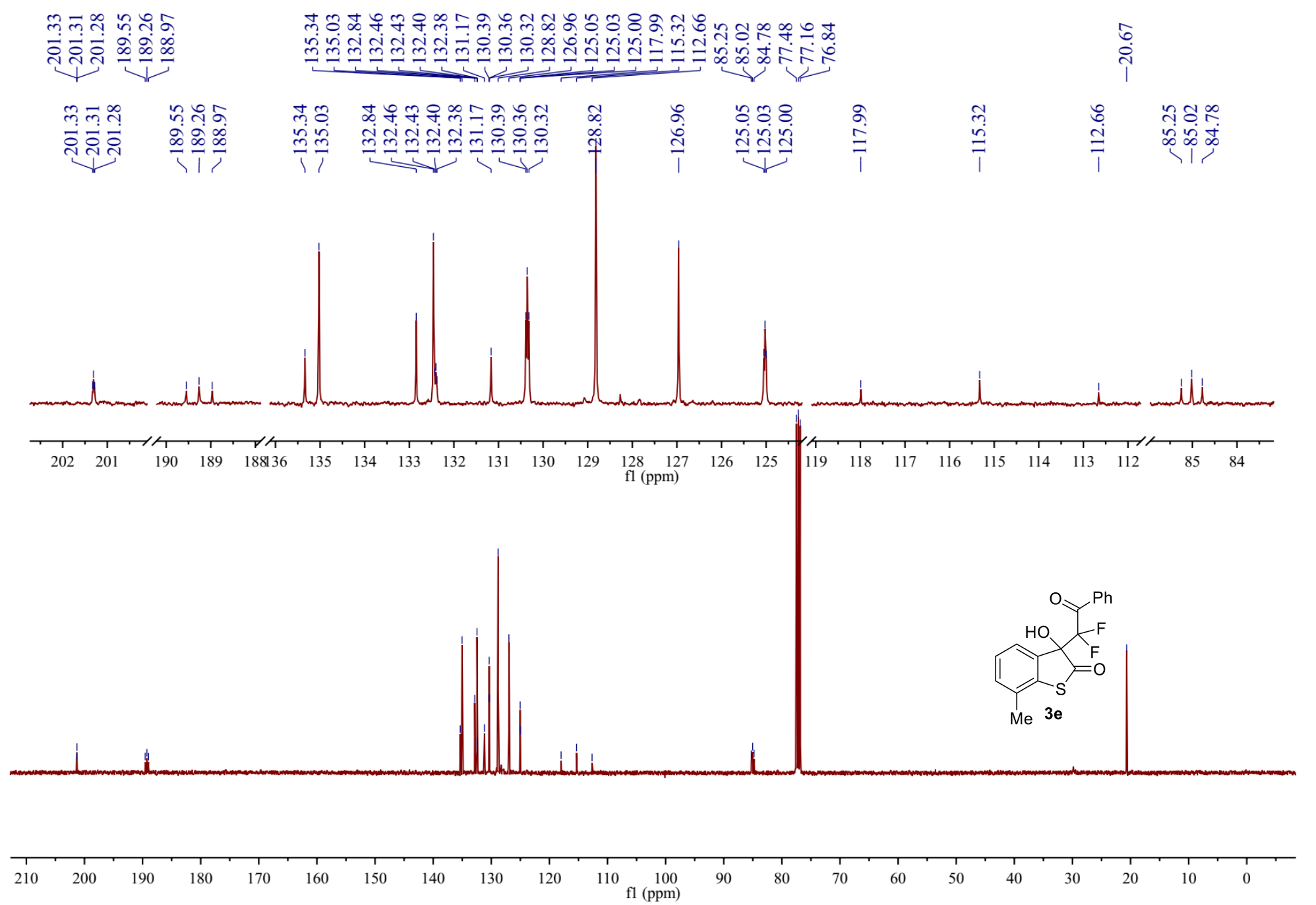

${ }^{13} \mathrm{C}\left\{{ }^{1} \mathrm{H}\right\}$ NMR spectra of $\mathbf{3 e}$ in $\mathrm{CDCl}_{3}(100 \mathrm{MHz})$ 


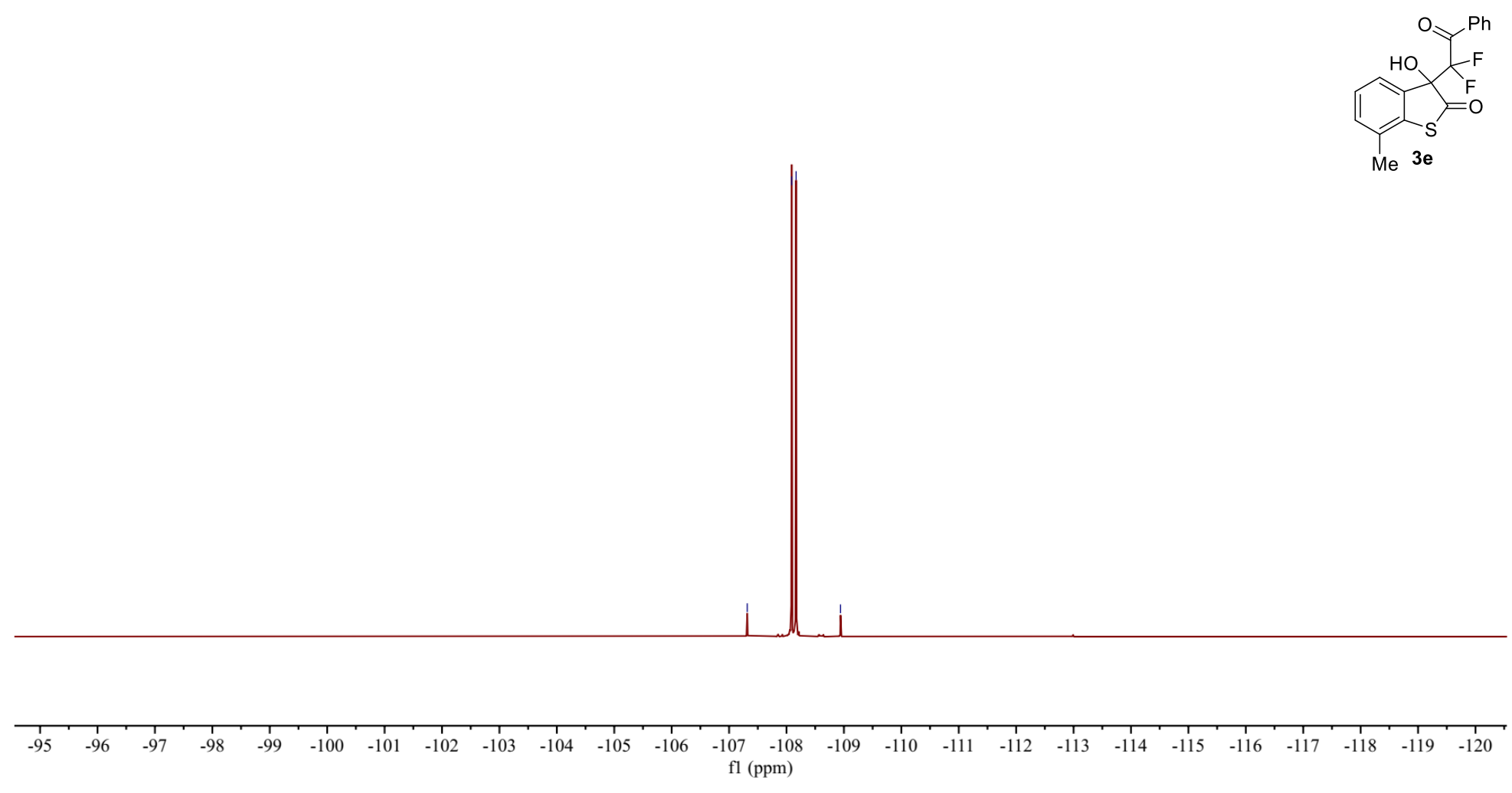

${ }^{19} \mathrm{~F}$ NMR spectra of $\mathbf{3 e}$ in $\mathrm{CDCl}_{3}(376 \mathrm{MHz})$ 

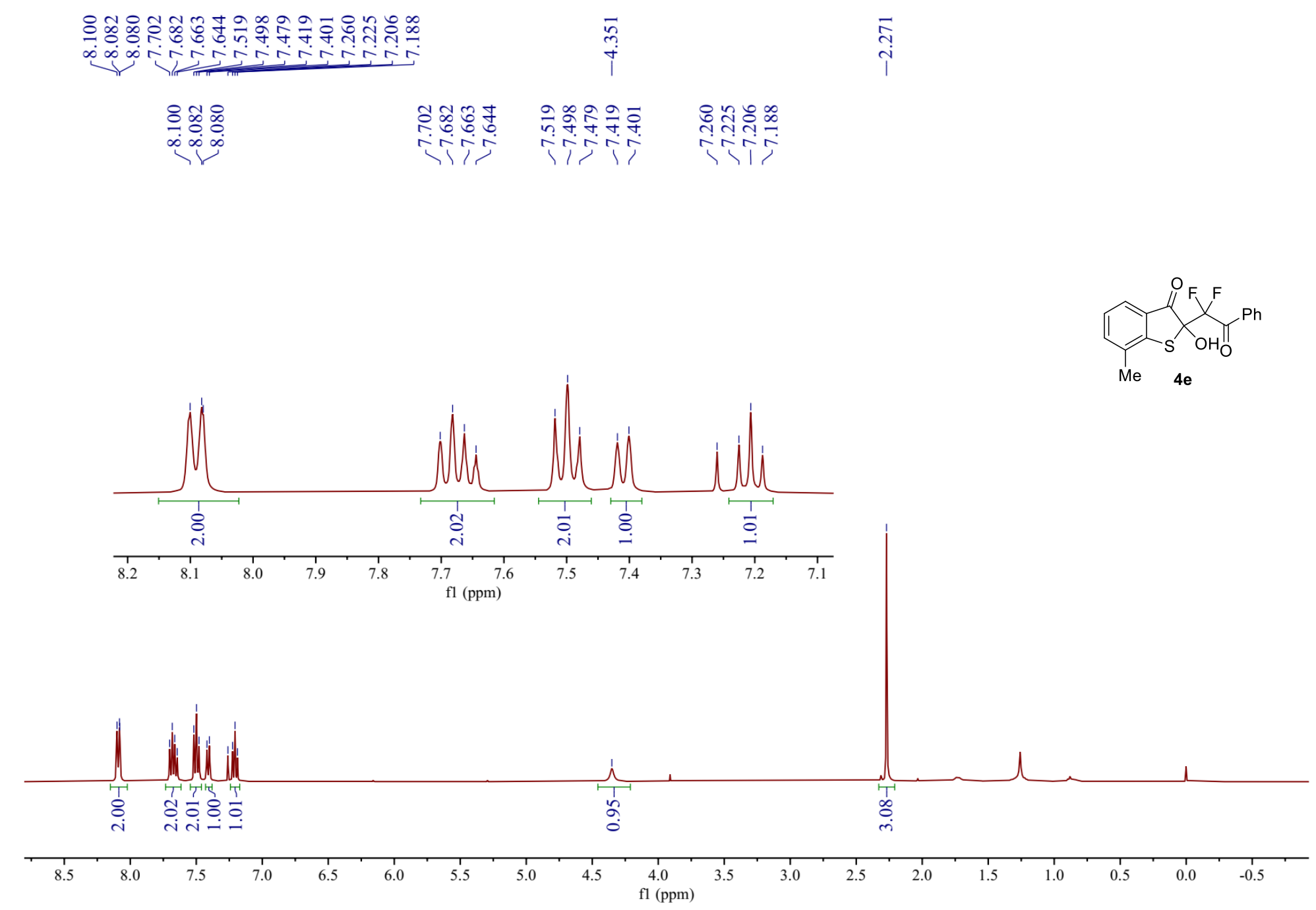

$\mathrm{Me} \quad 4 \mathrm{e}$

${ }^{1} \mathrm{H}$ NMR spectra of $\mathbf{4 e}$ in $\mathrm{CDCl}_{3}(400 \mathrm{MHz})$ 


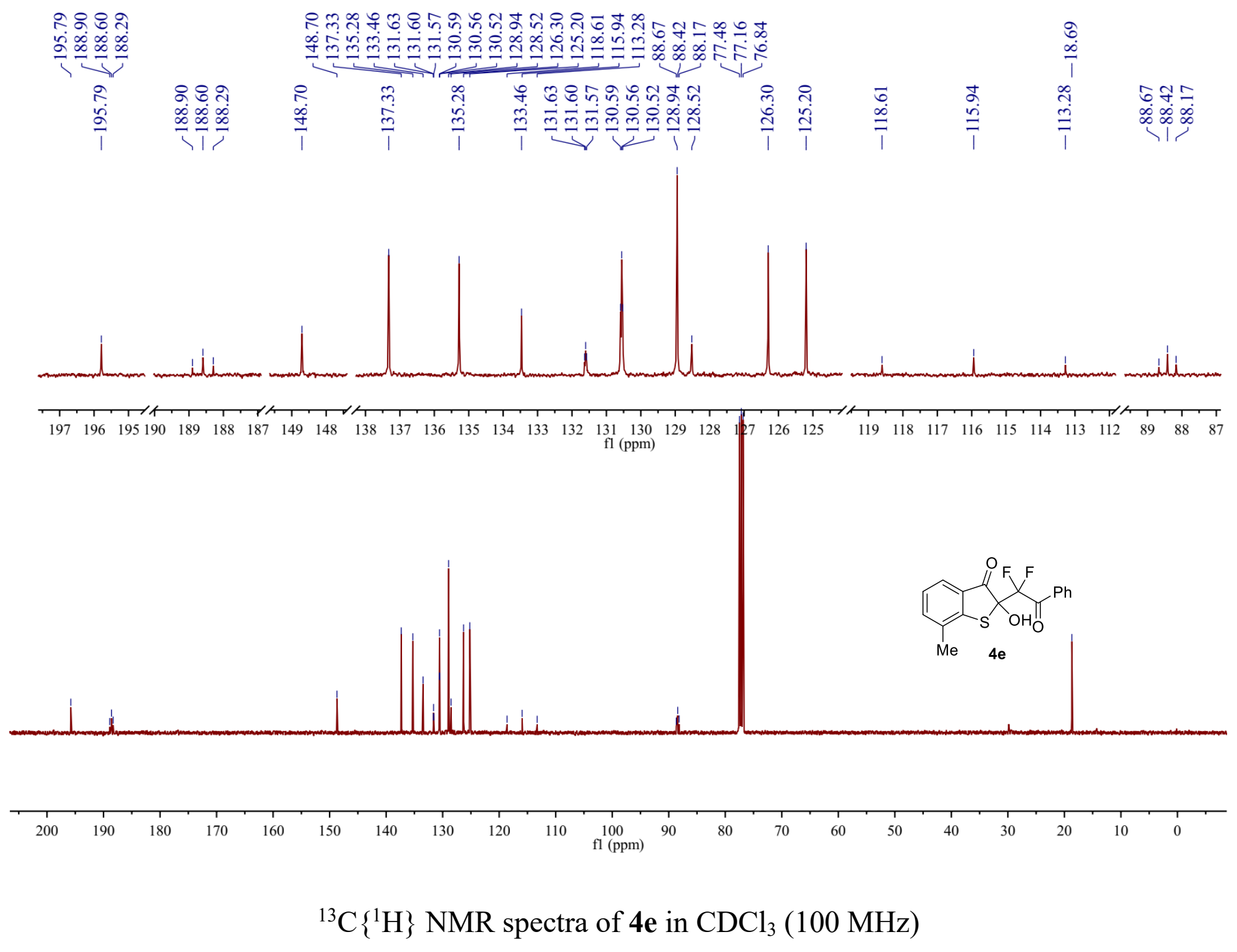




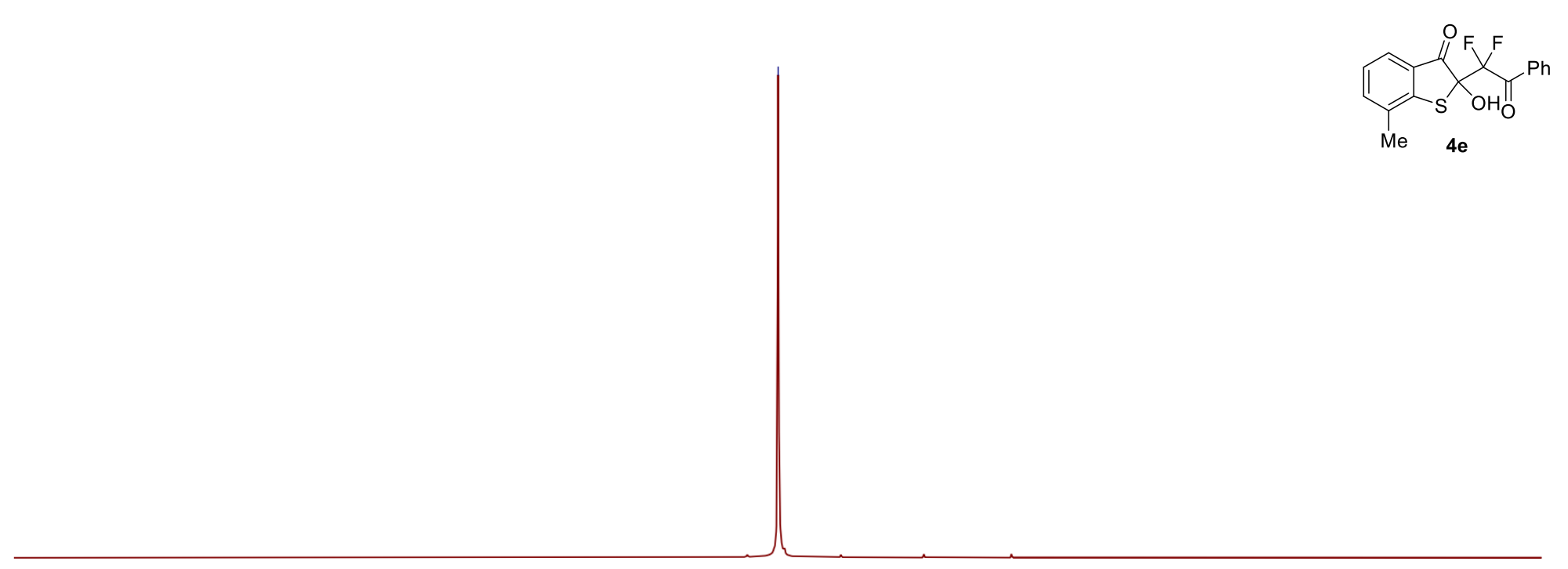

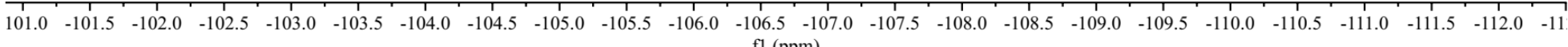
$\mathrm{fl}(\mathrm{ppm})$

${ }^{19} \mathrm{~F} \mathrm{NMR} \mathrm{spectra} \mathrm{of} \mathbf{4 e}$ in $\mathrm{CDCl}_{3}(376 \mathrm{MHz})$ 


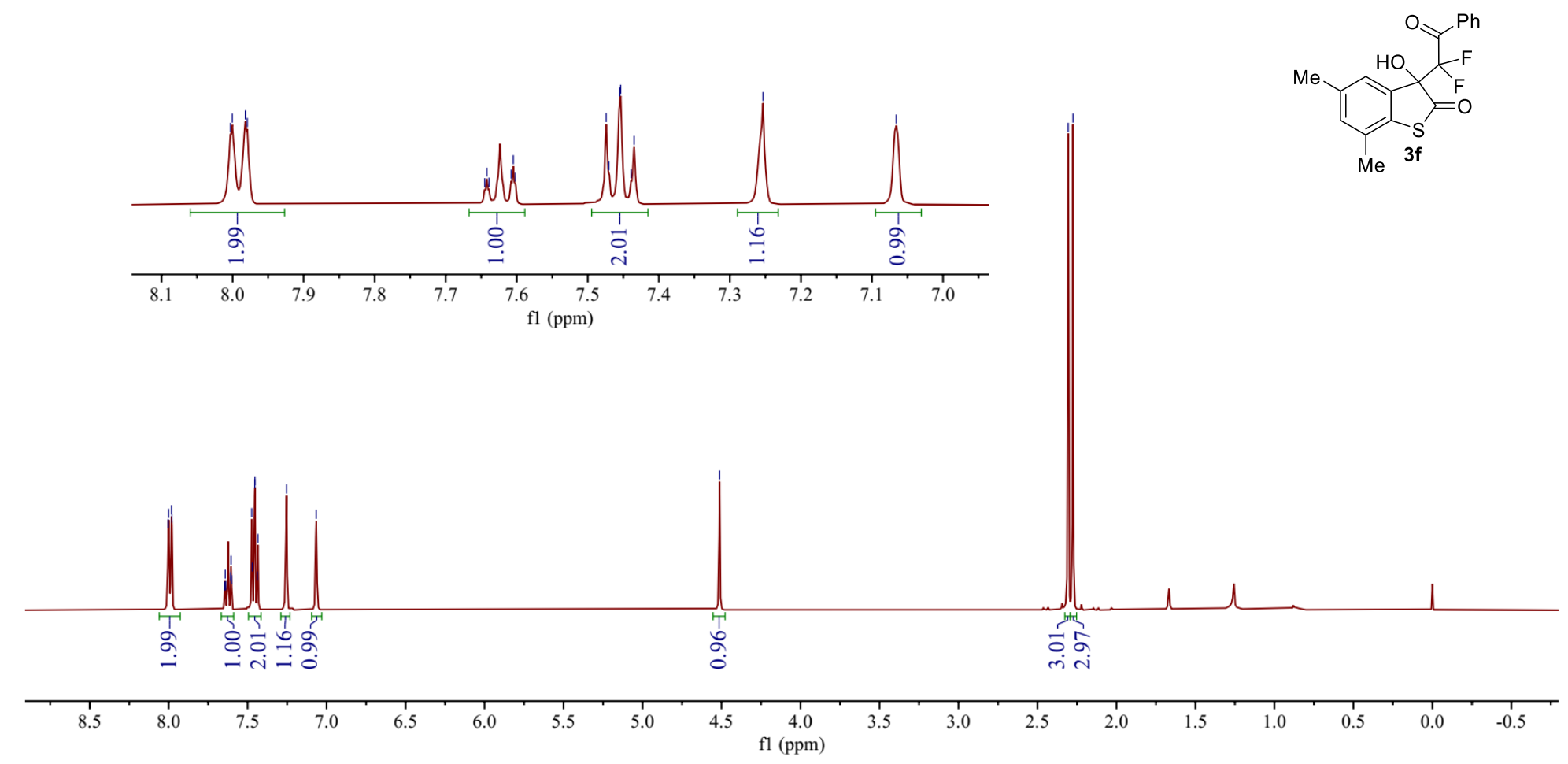

${ }^{1} \mathrm{H}$ NMR spectra of $\mathbf{3 f}$ in $\mathrm{CDCl}_{3}(400 \mathrm{MHz})$ 

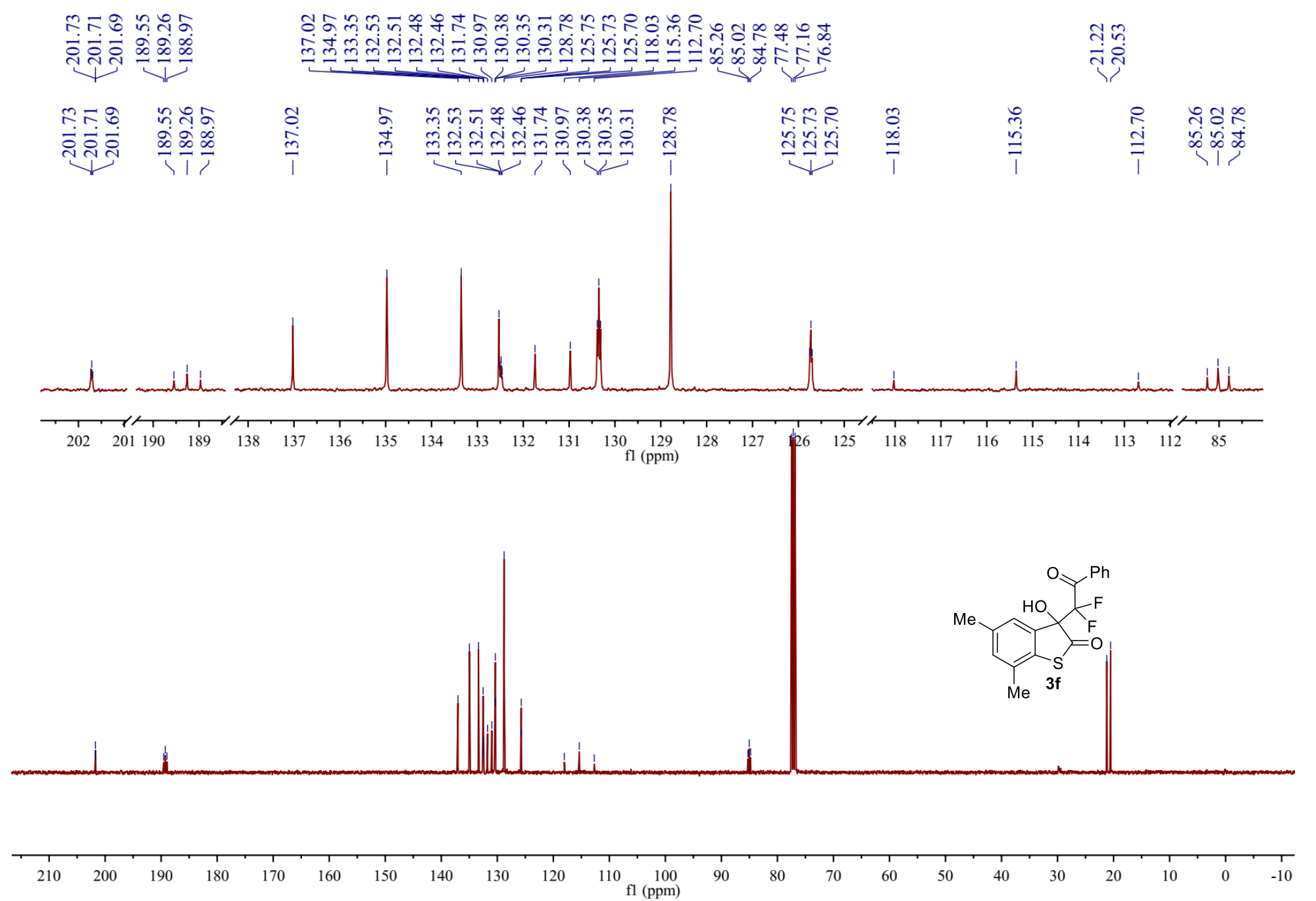

${ }^{13} \mathrm{C}\left\{{ }^{1} \mathrm{H}\right\}$ NMR spectra of $\mathbf{3 f}$ in $\mathrm{CDCl}_{3}(100 \mathrm{MHz})$ 


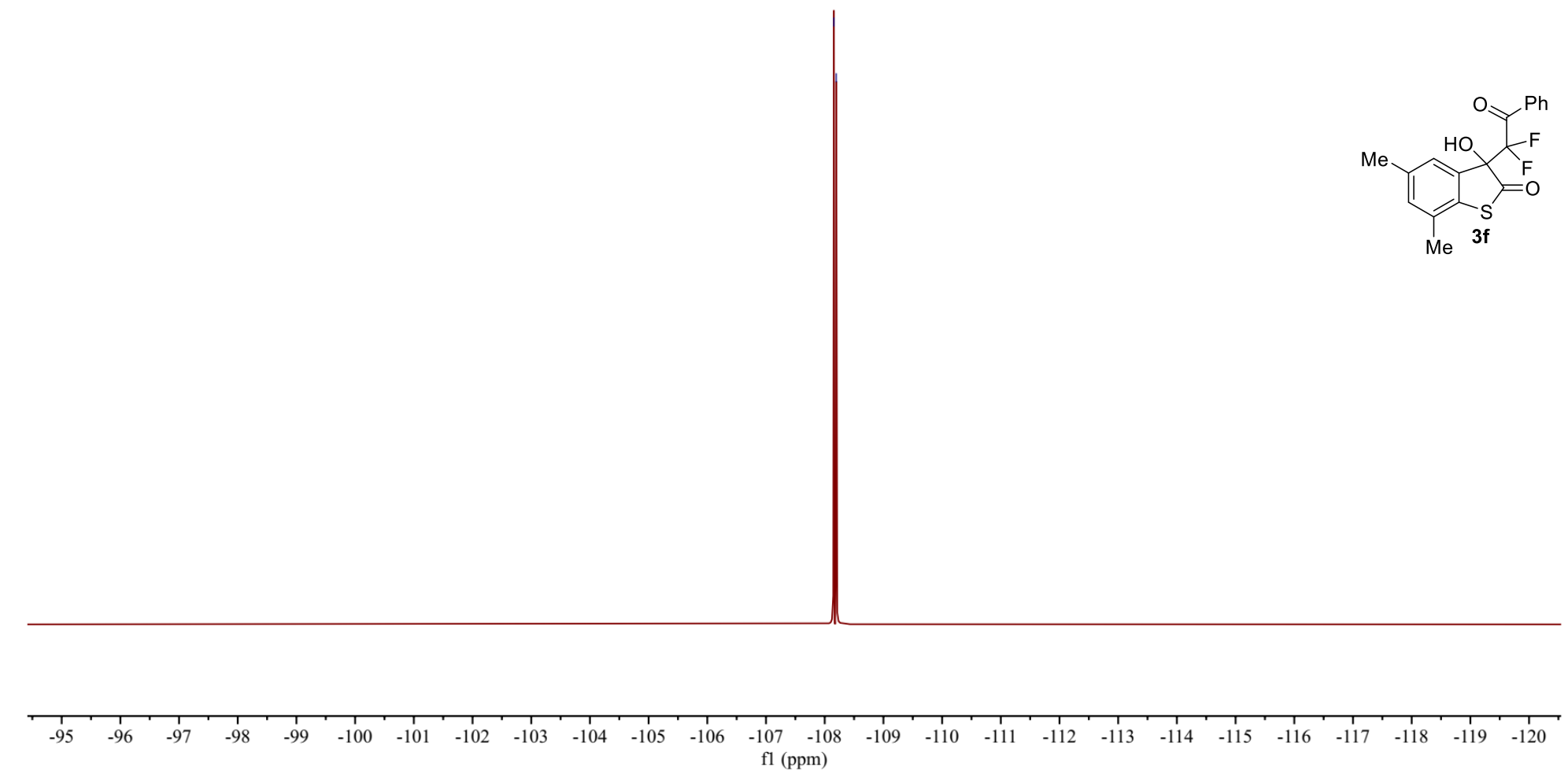

${ }^{19} \mathrm{~F}$ NMR spectra of $\mathbf{3 f}$ in $\mathrm{CDCl}_{3}(376 \mathrm{MHz})$ 


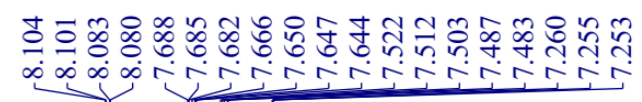

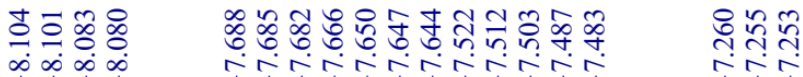

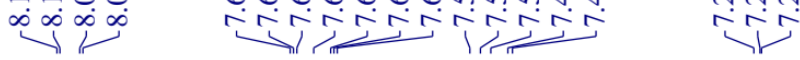
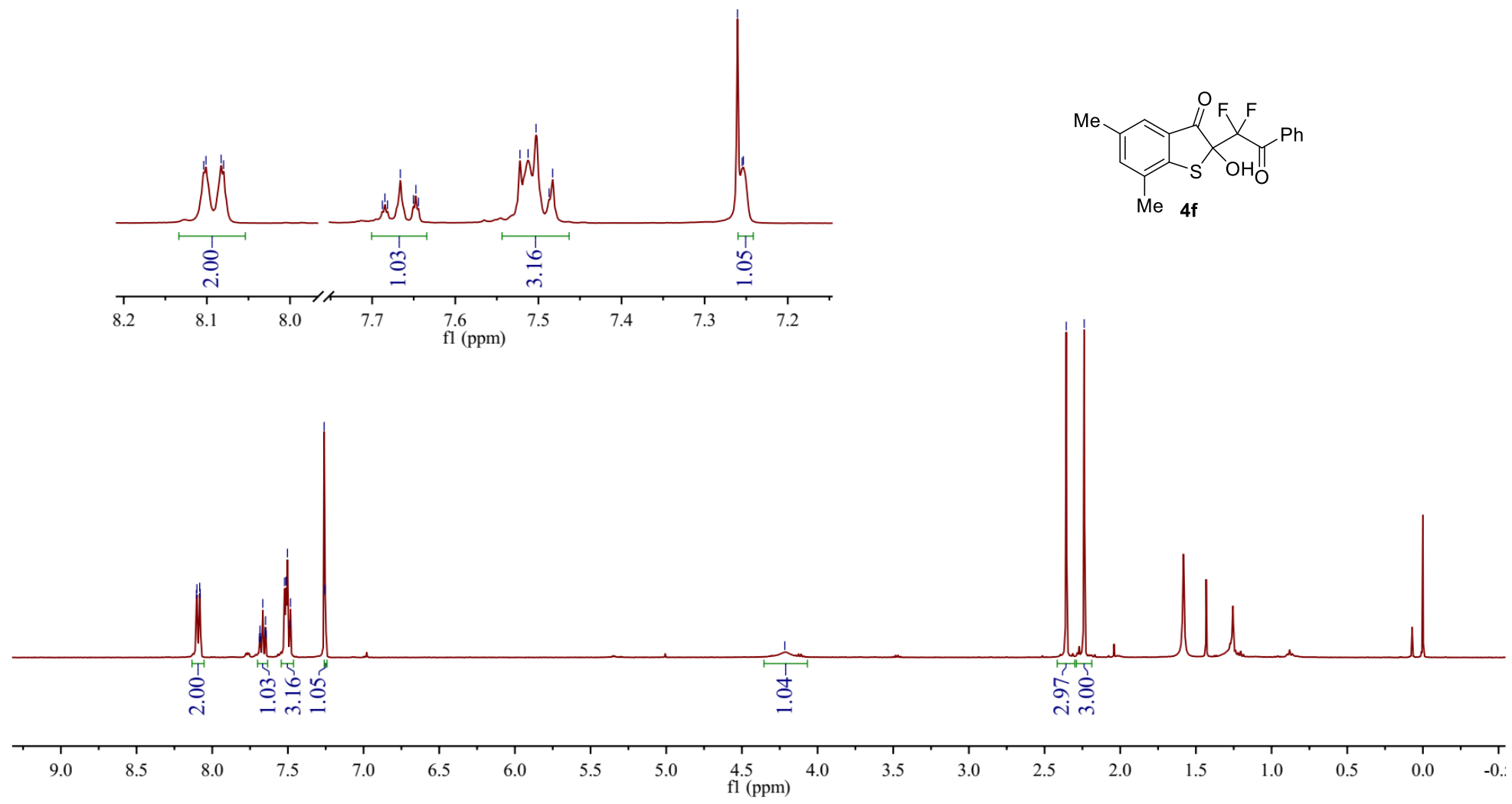

${ }^{1} \mathrm{H}$ NMR spectra of $\mathbf{4 f}$ in $\mathrm{CDCl}_{3}(400 \mathrm{MHz})$ 

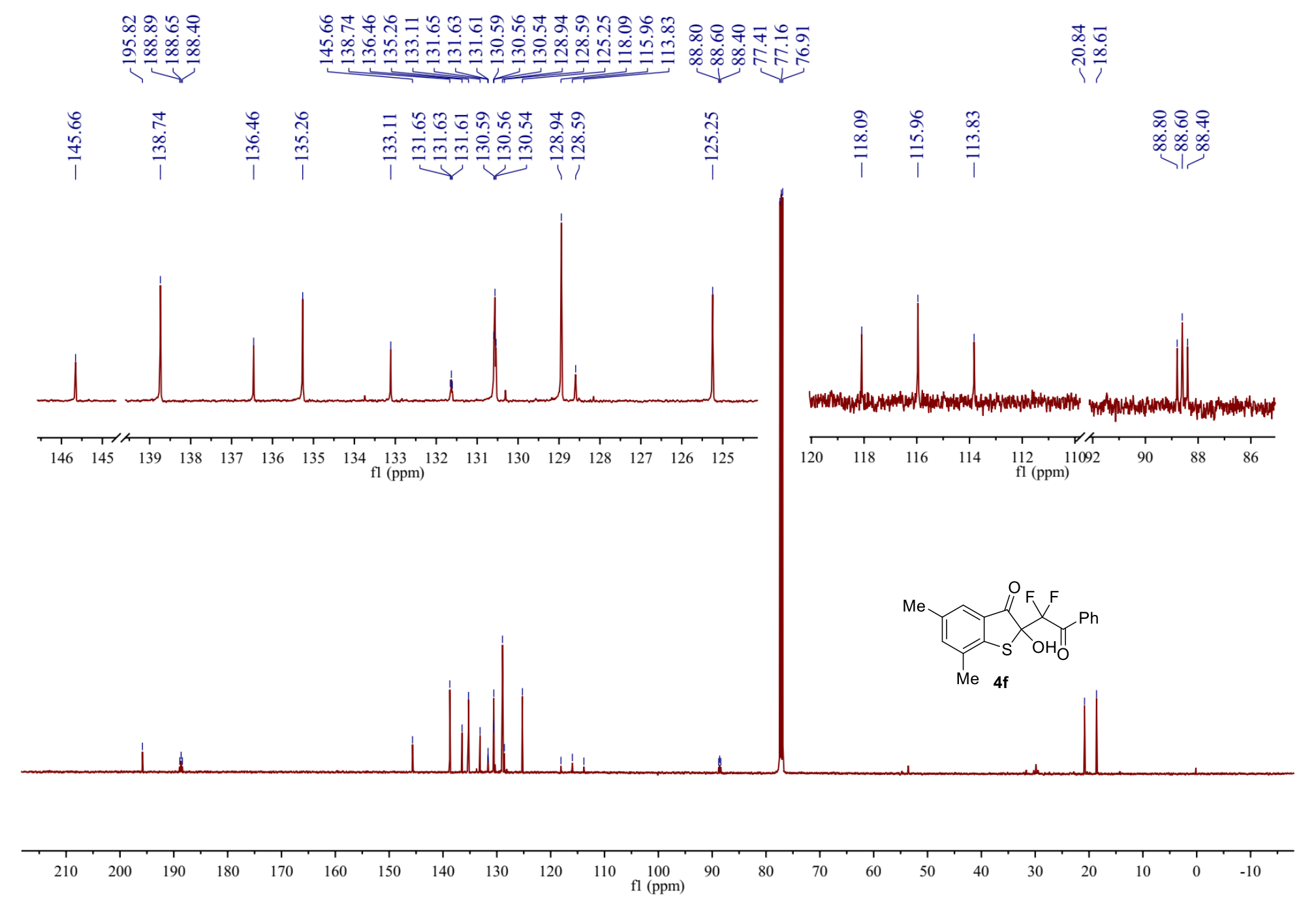

${ }^{13} \mathrm{C}\left\{{ }^{1} \mathrm{H}\right\}$ NMR spectra of $\mathbf{4 f}$ in $\mathrm{CDCl}_{3}(125 \mathrm{MHz})$ 


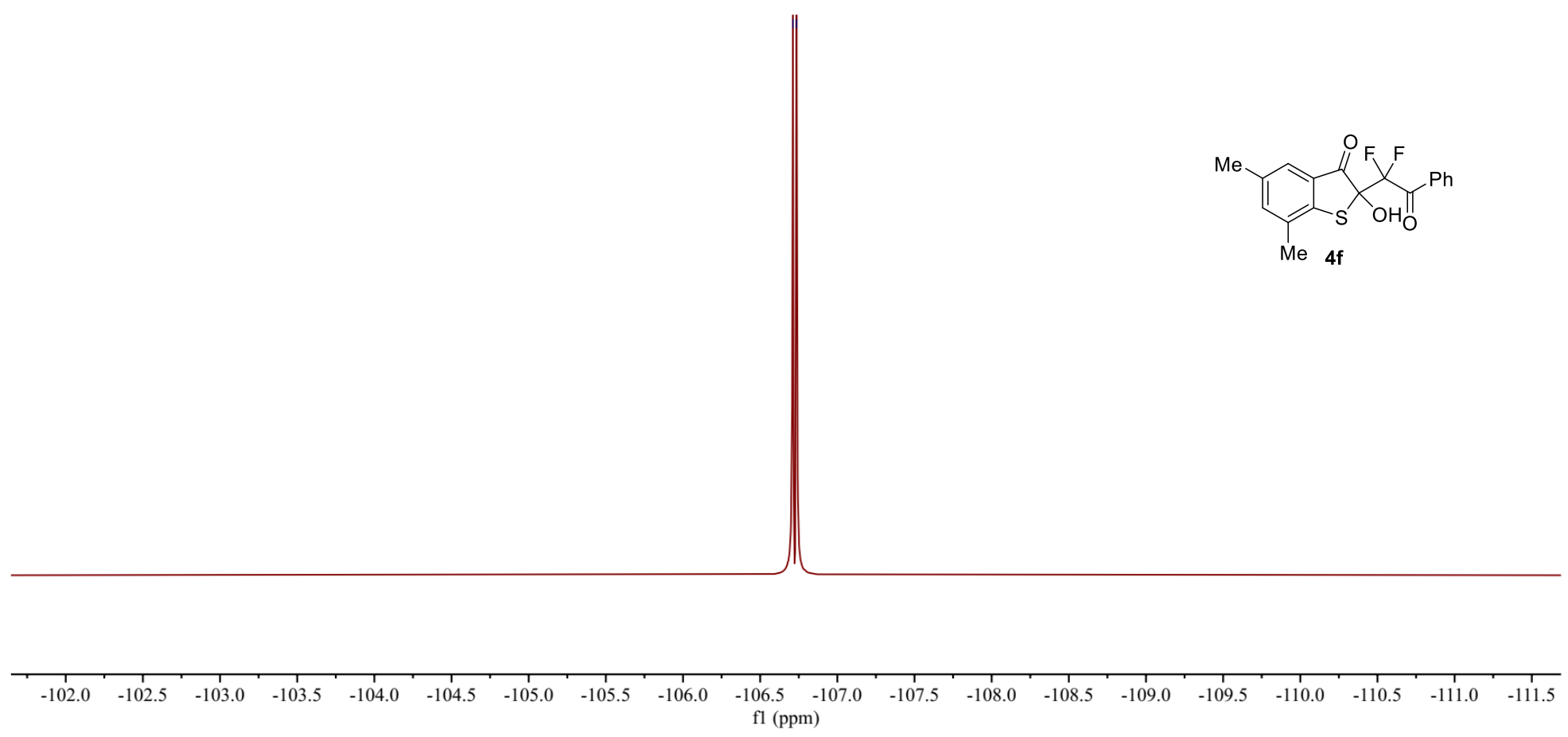

${ }^{19} \mathrm{~F}$ NMR spectra of $\mathbf{4 f}$ in $\mathrm{CDCl}_{3}(376 \mathrm{MHz})$ 


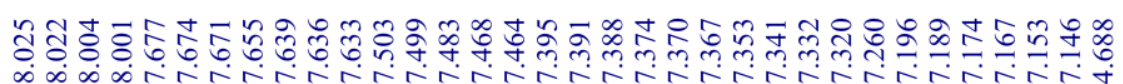

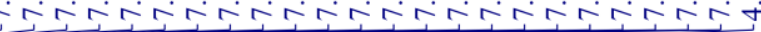
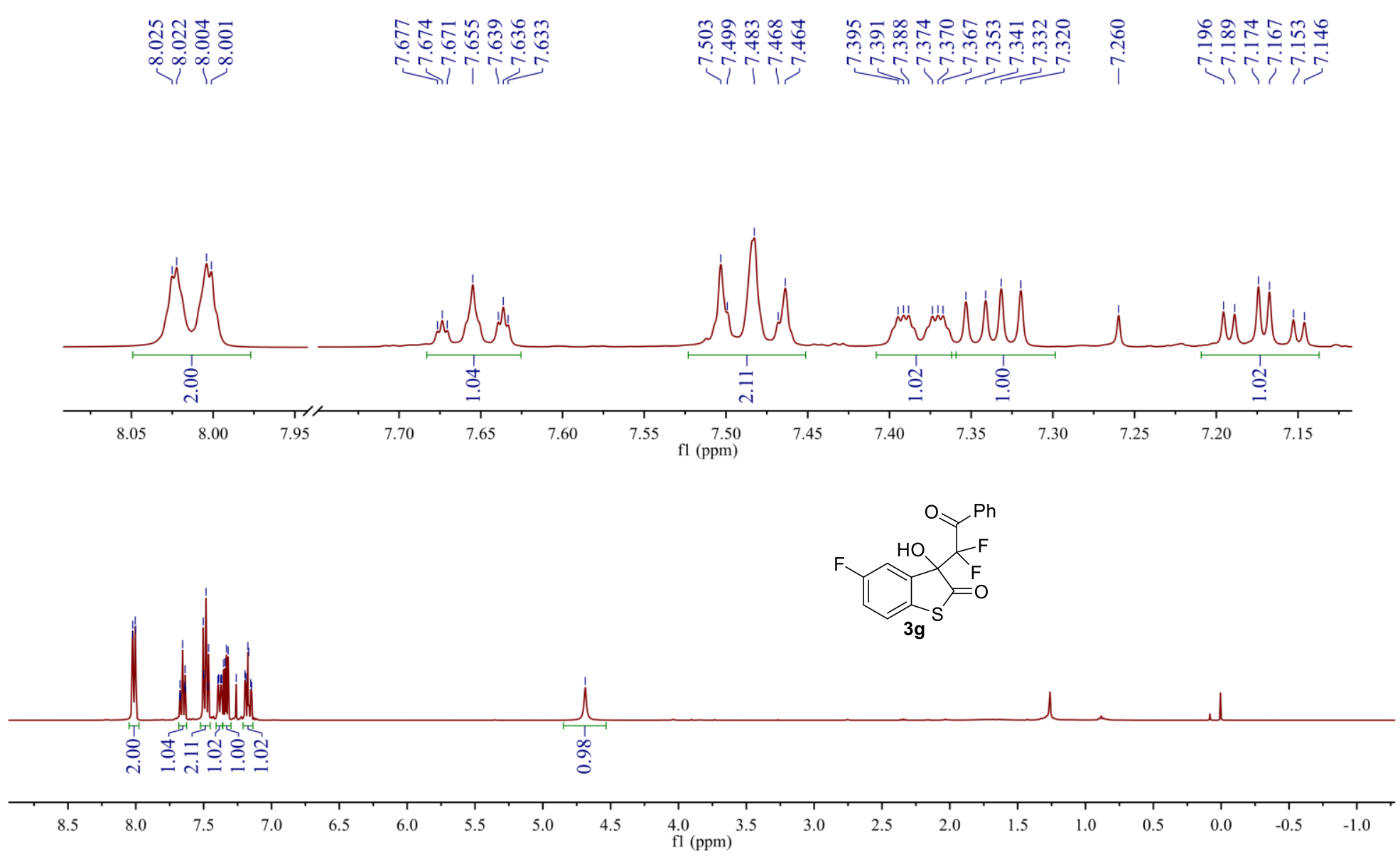

${ }^{1} \mathrm{H}$ NMR spectra of $\mathbf{3 g}$ in $\mathrm{CDCl}_{3}(400 \mathrm{MHz})$ 

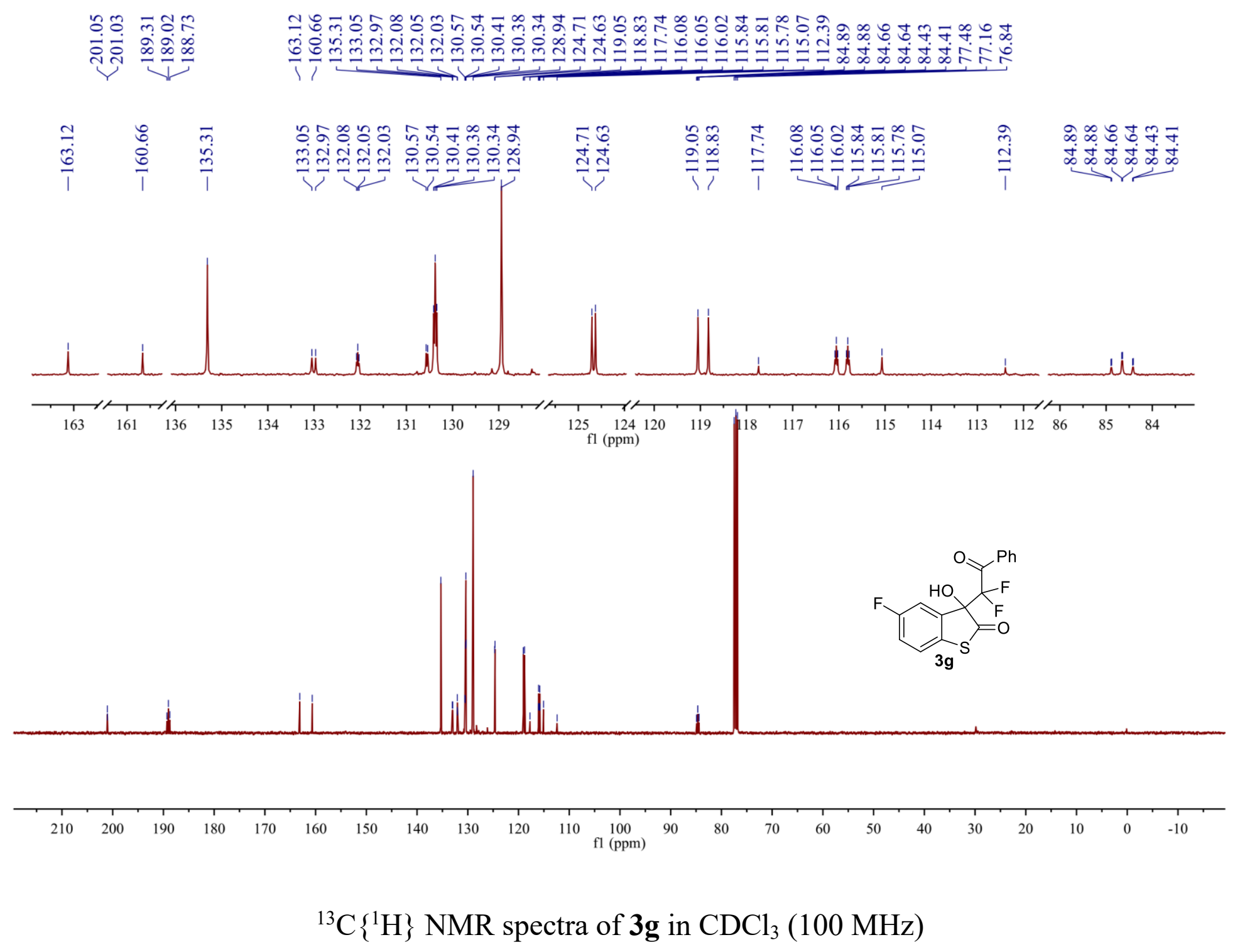


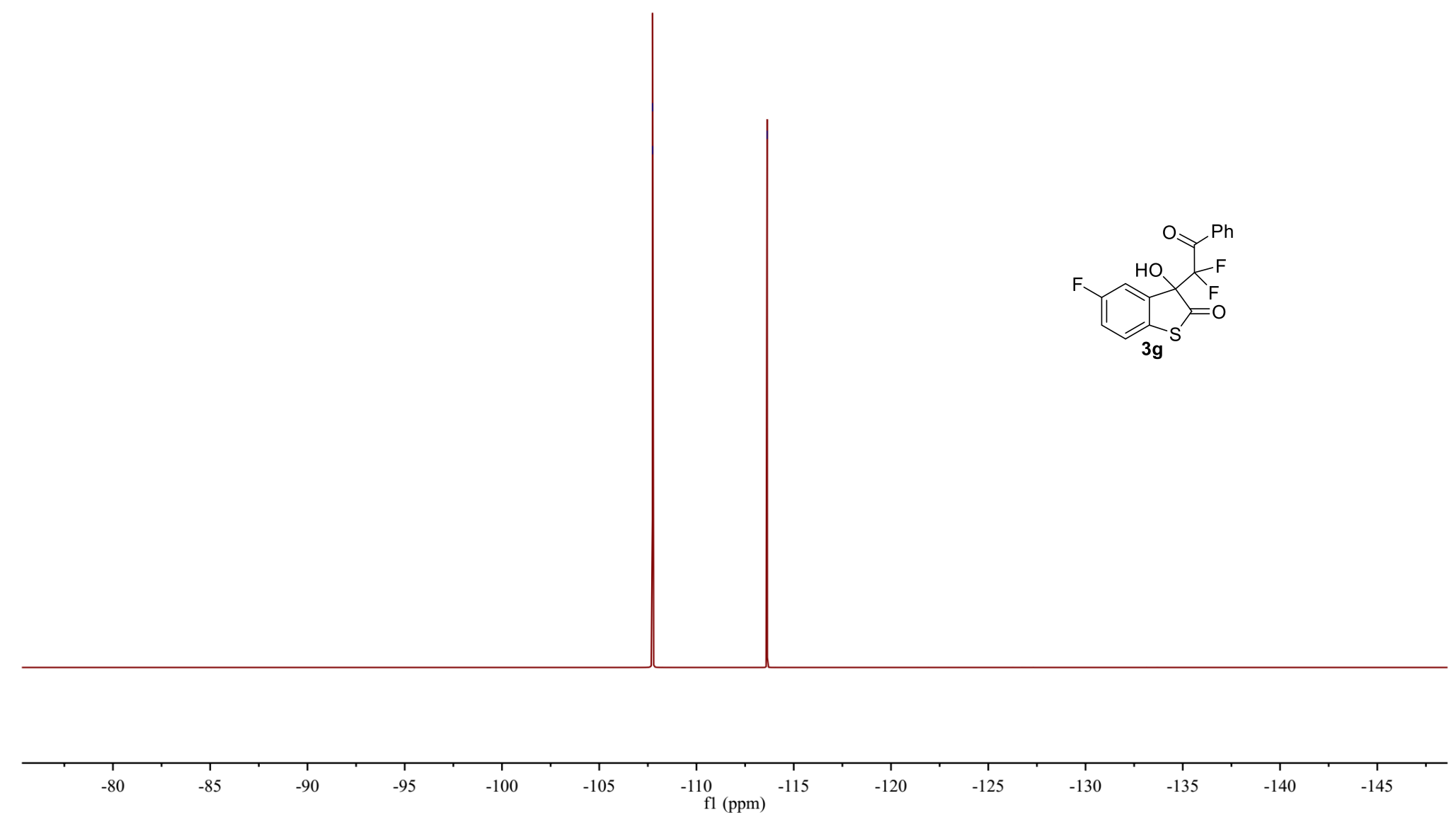

${ }^{19} \mathrm{~F}$ NMR spectra of $\mathbf{3 g}$ in $\mathrm{CDCl}_{3}(376 \mathrm{MHz})$ 


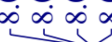

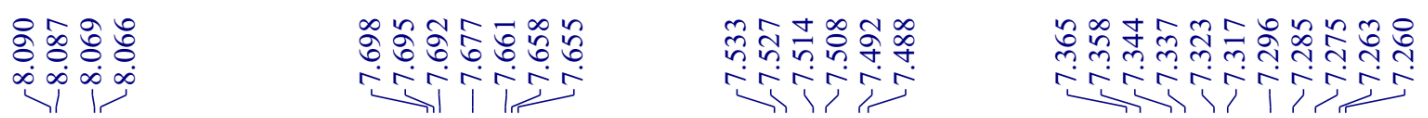
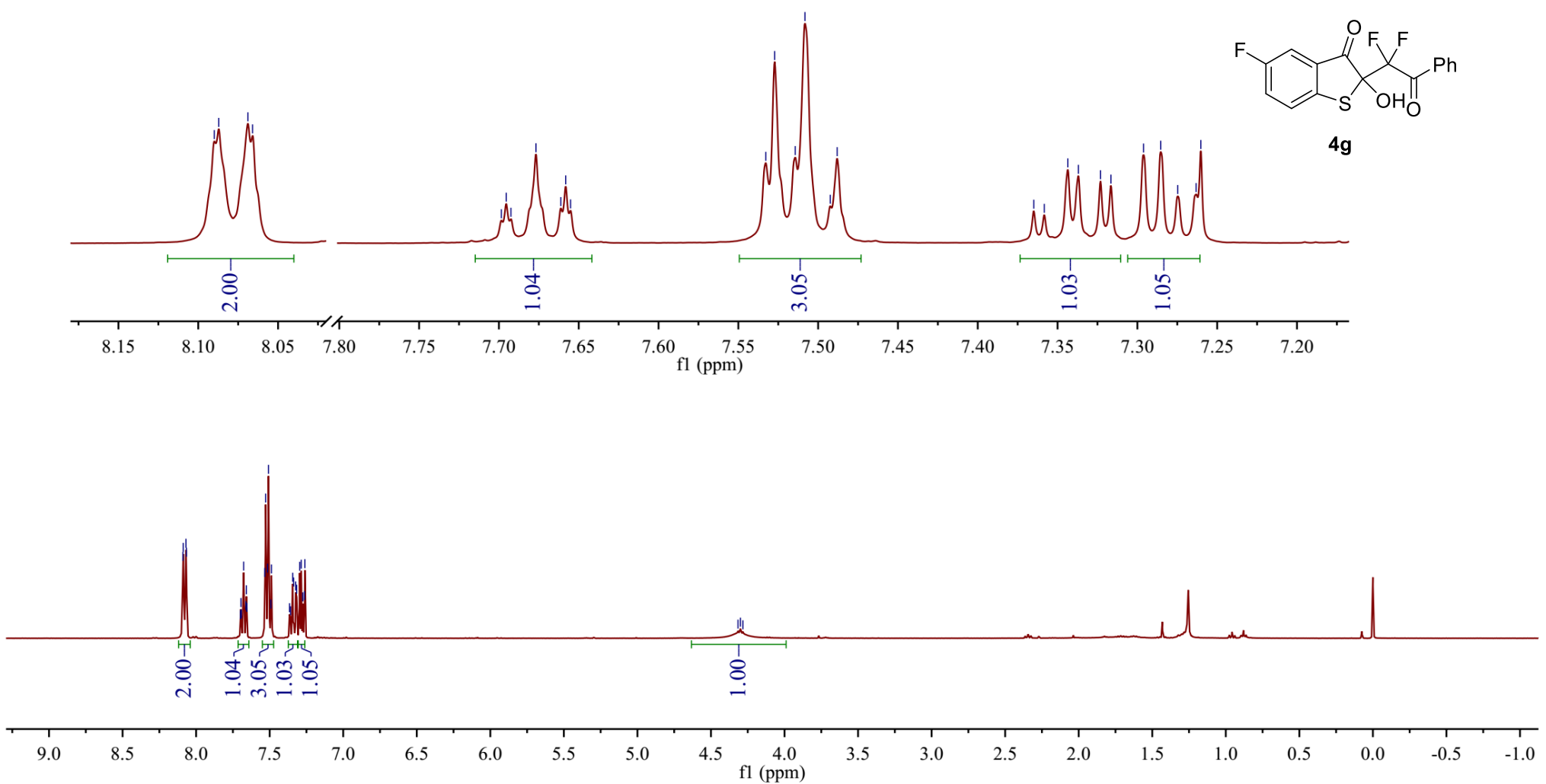

${ }^{1} \mathrm{H}$ NMR spectra of $\mathbf{4 g}$ in $\mathrm{CDCl}_{3}(400 \mathrm{MHz})$ 

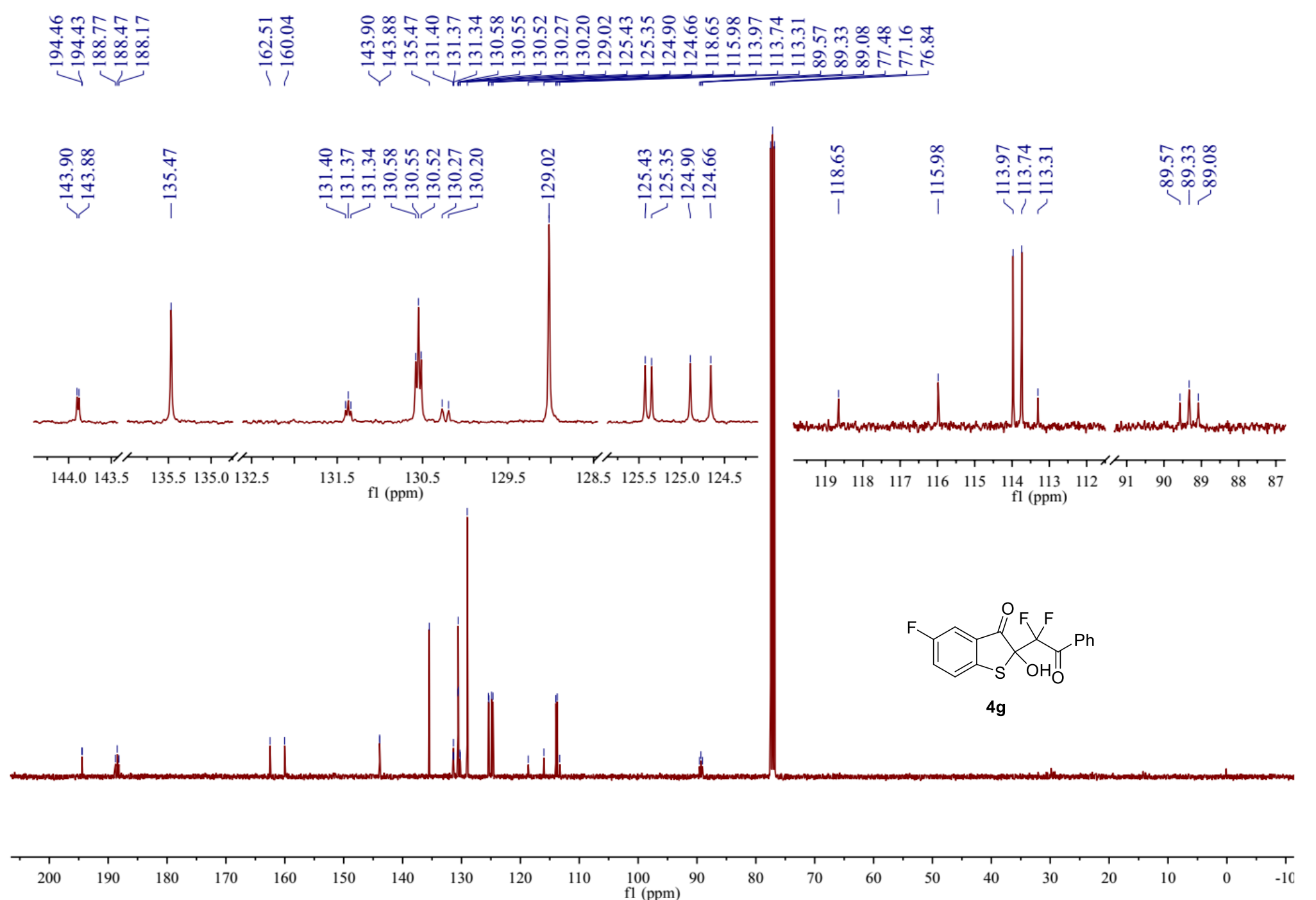

${ }^{13} \mathrm{C}\left\{{ }^{1} \mathrm{H}\right\}$ NMR spectra of $\mathbf{4 g}$ in $\mathrm{CDCl}_{3}(100 \mathrm{MHz})$ 


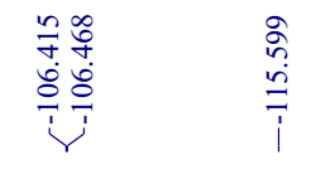

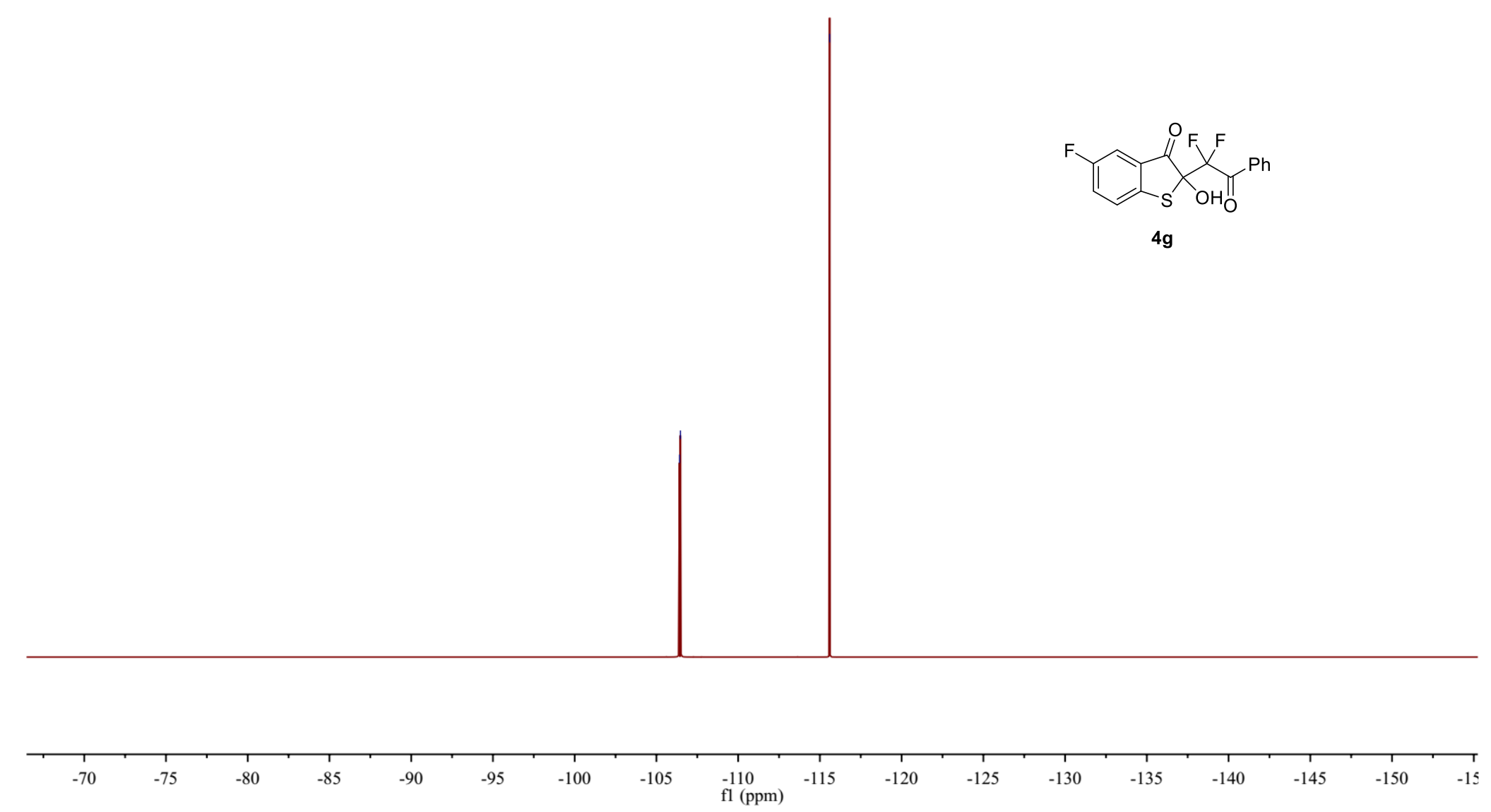

${ }^{19} \mathrm{~F}$ NMR spectra of $\mathbf{4 g}$ in $\mathrm{CDCl}_{3}(376 \mathrm{MHz})$ 


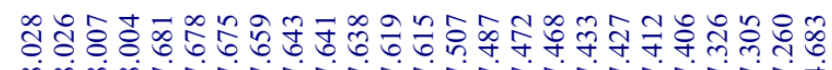

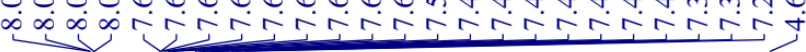

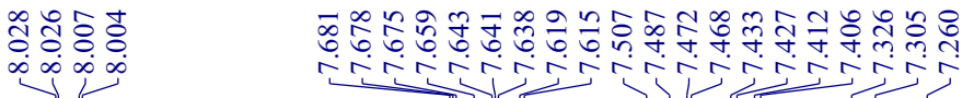
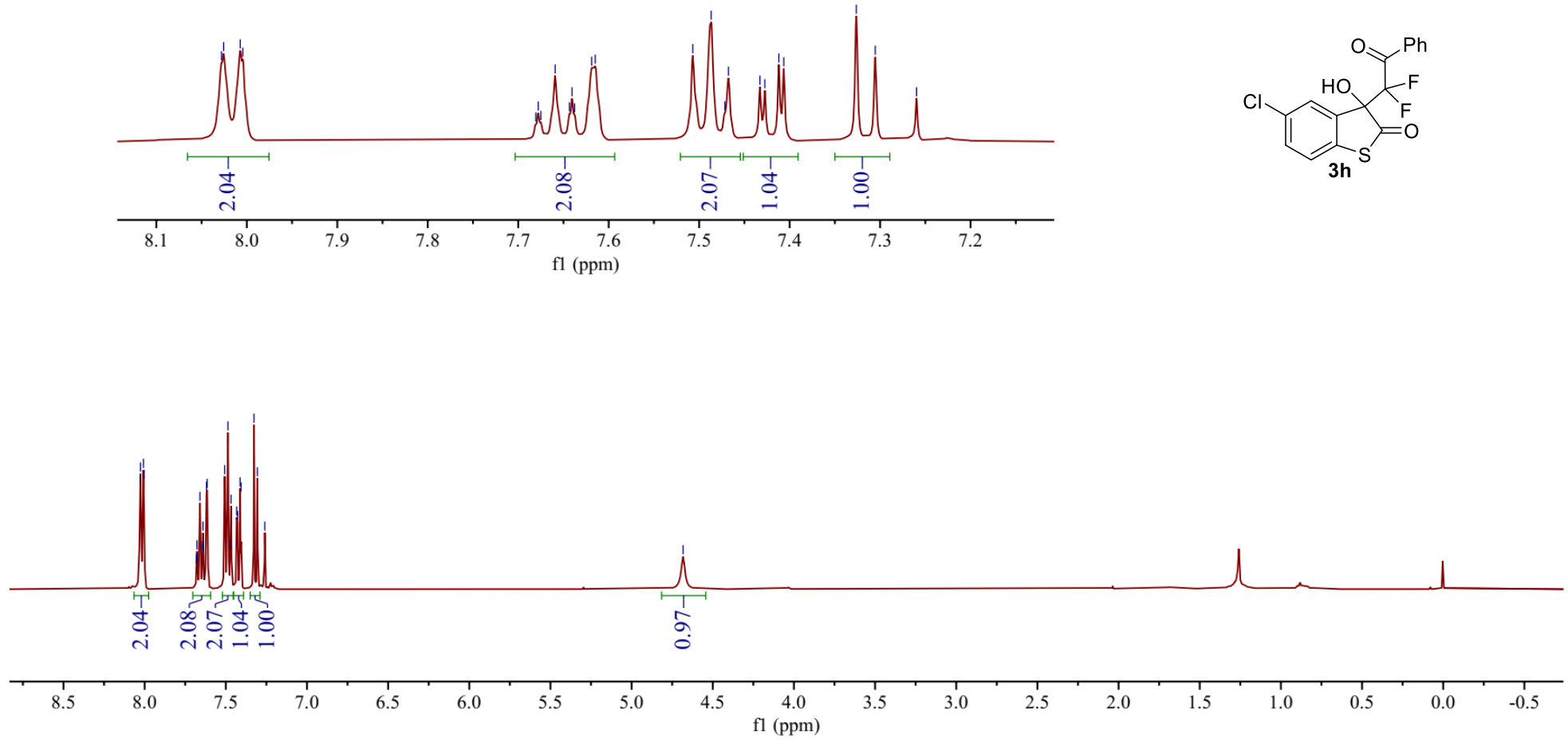

${ }^{1} \mathrm{H}$ NMR spectra of $\mathbf{3 h}$ in $\mathrm{CDCl}_{3}(400 \mathrm{MHz})$ 

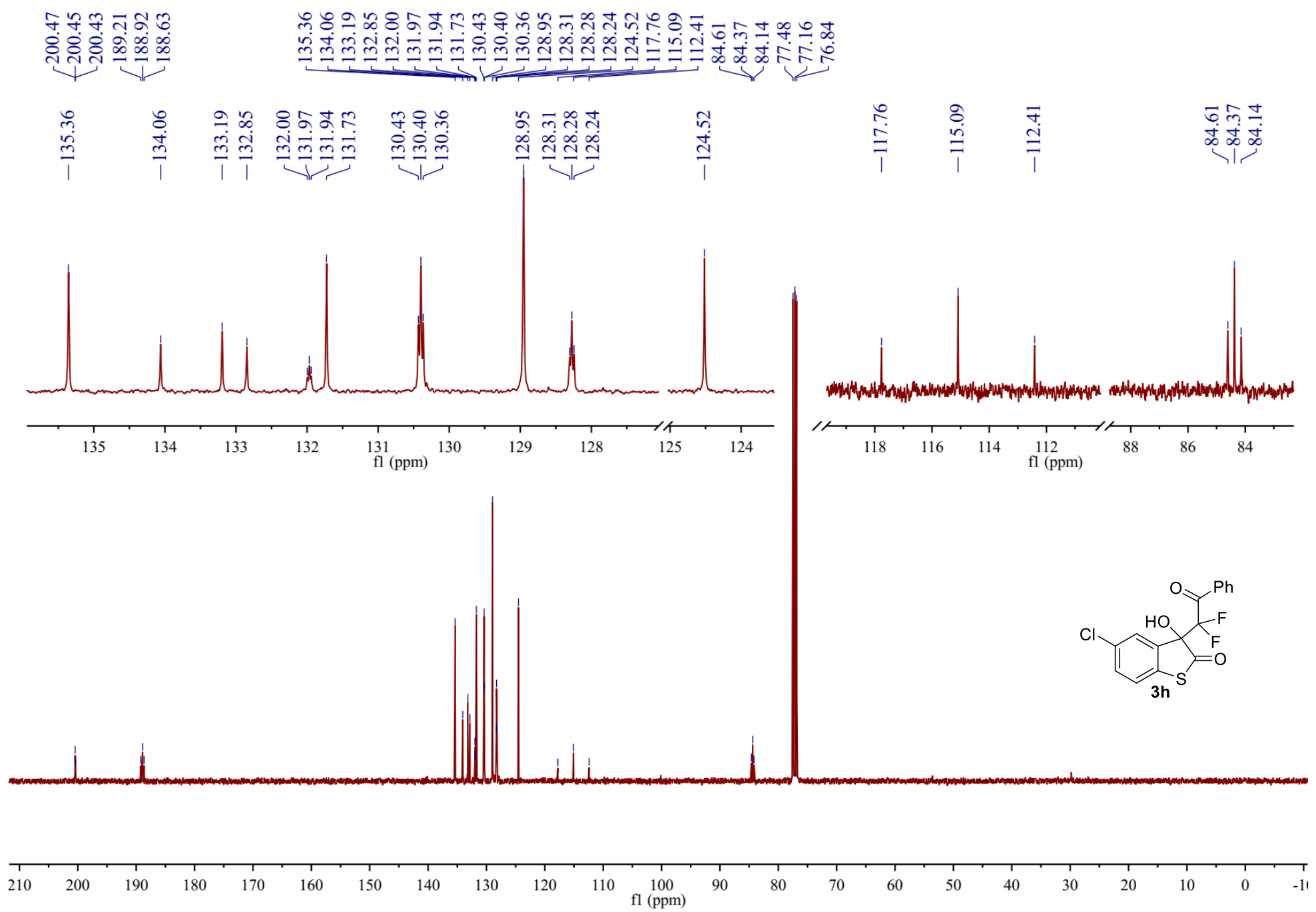

${ }^{13} \mathrm{C}\left\{{ }^{1} \mathrm{H}\right\}$ NMR spectra of $\mathbf{3 h}$ in $\mathrm{CDCl}_{3}(100 \mathrm{MHz})$ 


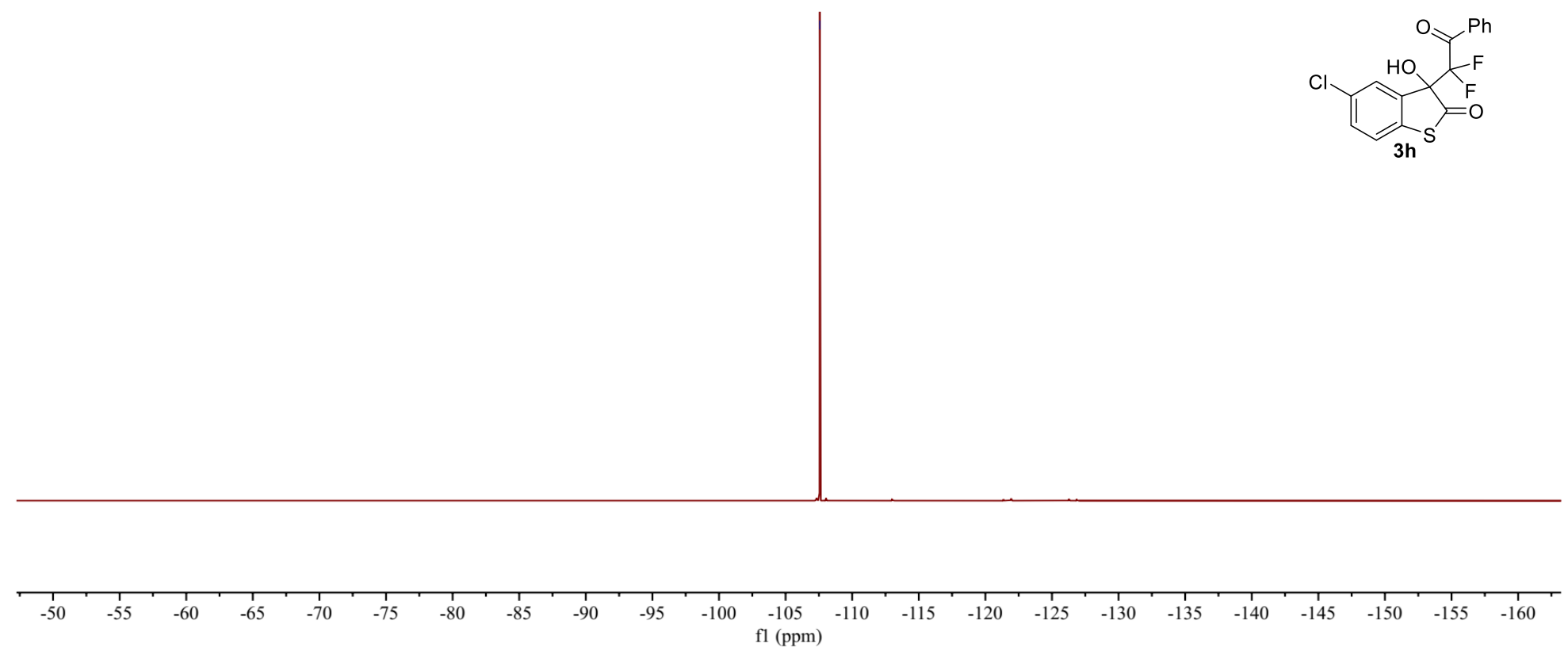

${ }^{19} \mathrm{~F}$ NMR spectra of $\mathbf{3 h}$ in $\mathrm{CDCl}_{3}(376 \mathrm{MHz})$ 


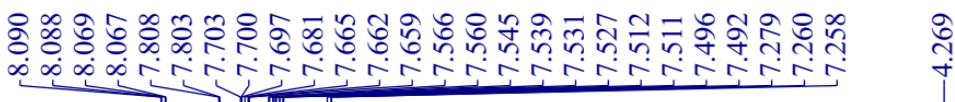

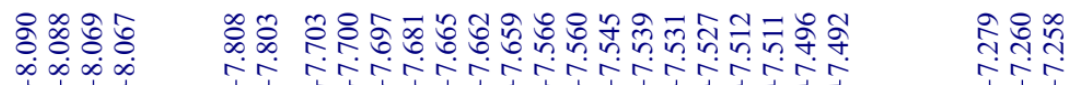

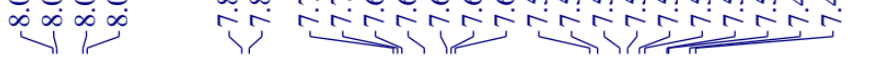
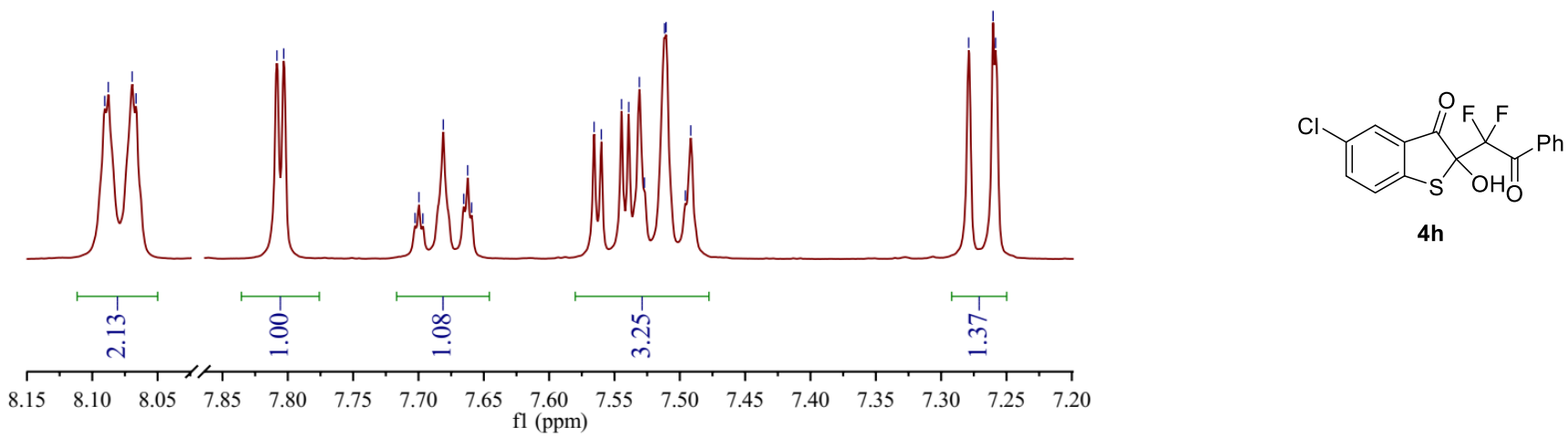

4h

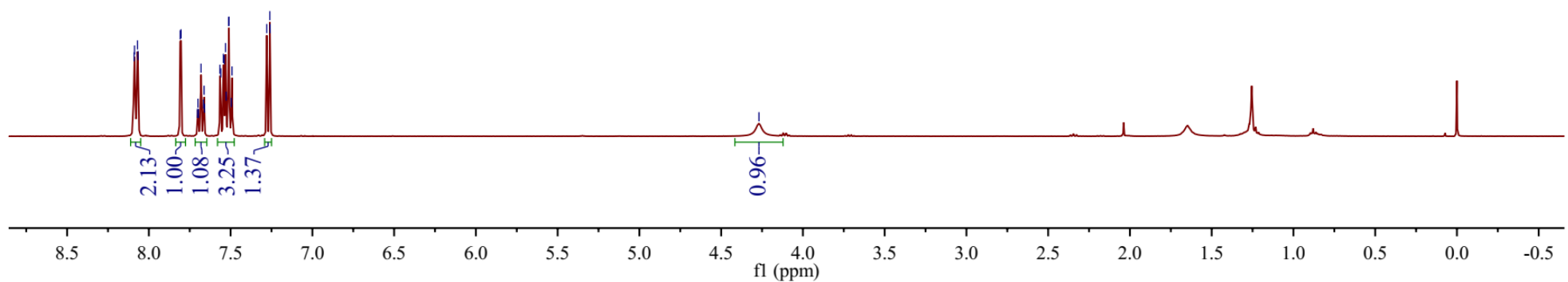

${ }^{1} \mathrm{H}$ NMR spectra of $\mathbf{4 h}$ in $\mathrm{CDCl}_{3}(400 \mathrm{MHz})$ 

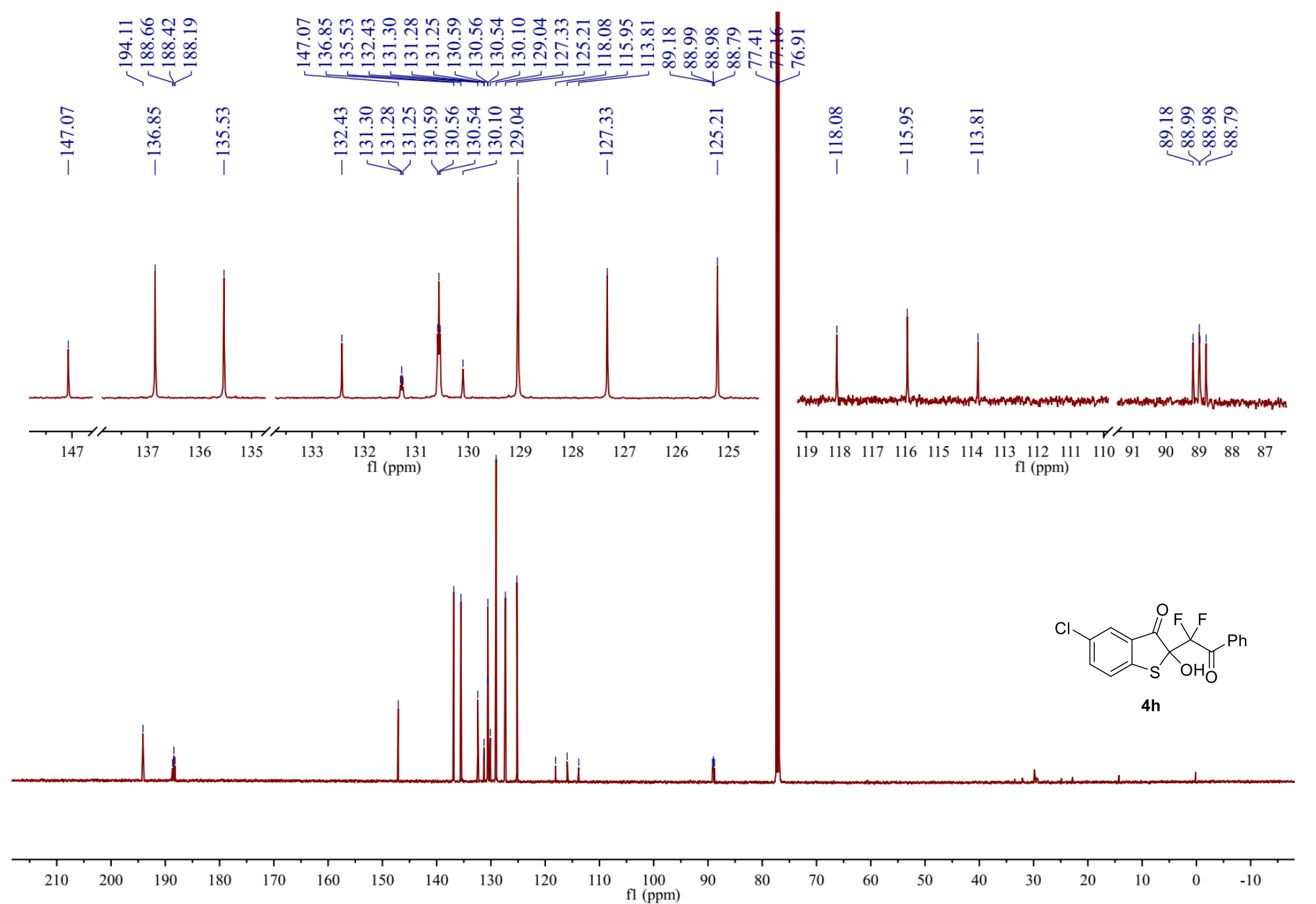

${ }^{13} \mathrm{C}\left\{{ }^{1} \mathrm{H}\right\}$ NMR spectra of $\mathbf{4 h}$ in $\mathrm{CDCl}_{3}(125 \mathrm{MHz})$ 


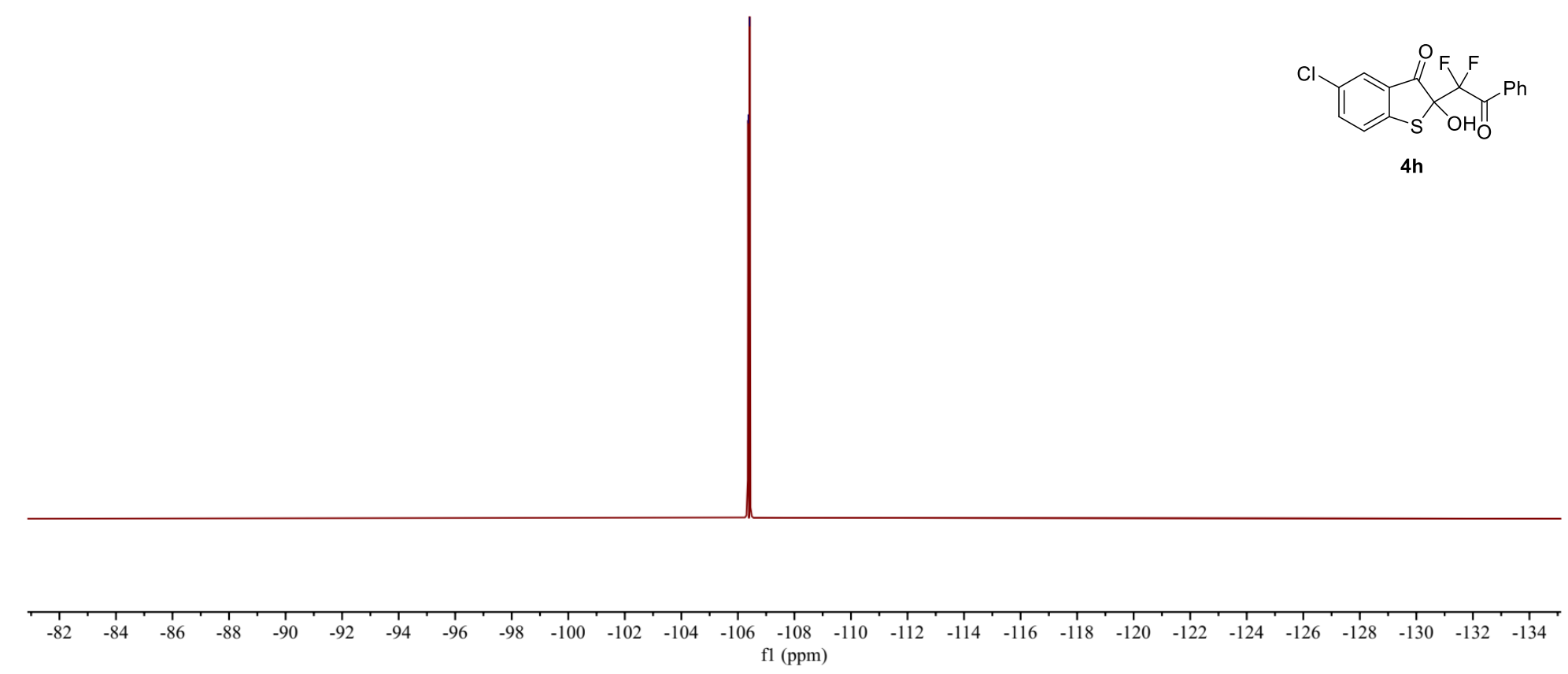

${ }^{19} \mathrm{~F}$ NMR spectra of $\mathbf{4 h}$ in $\mathrm{CDCl}_{3}(376 \mathrm{MHz})$ 


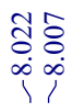

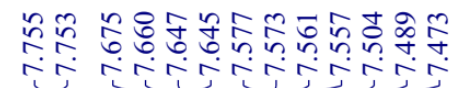

ริำด
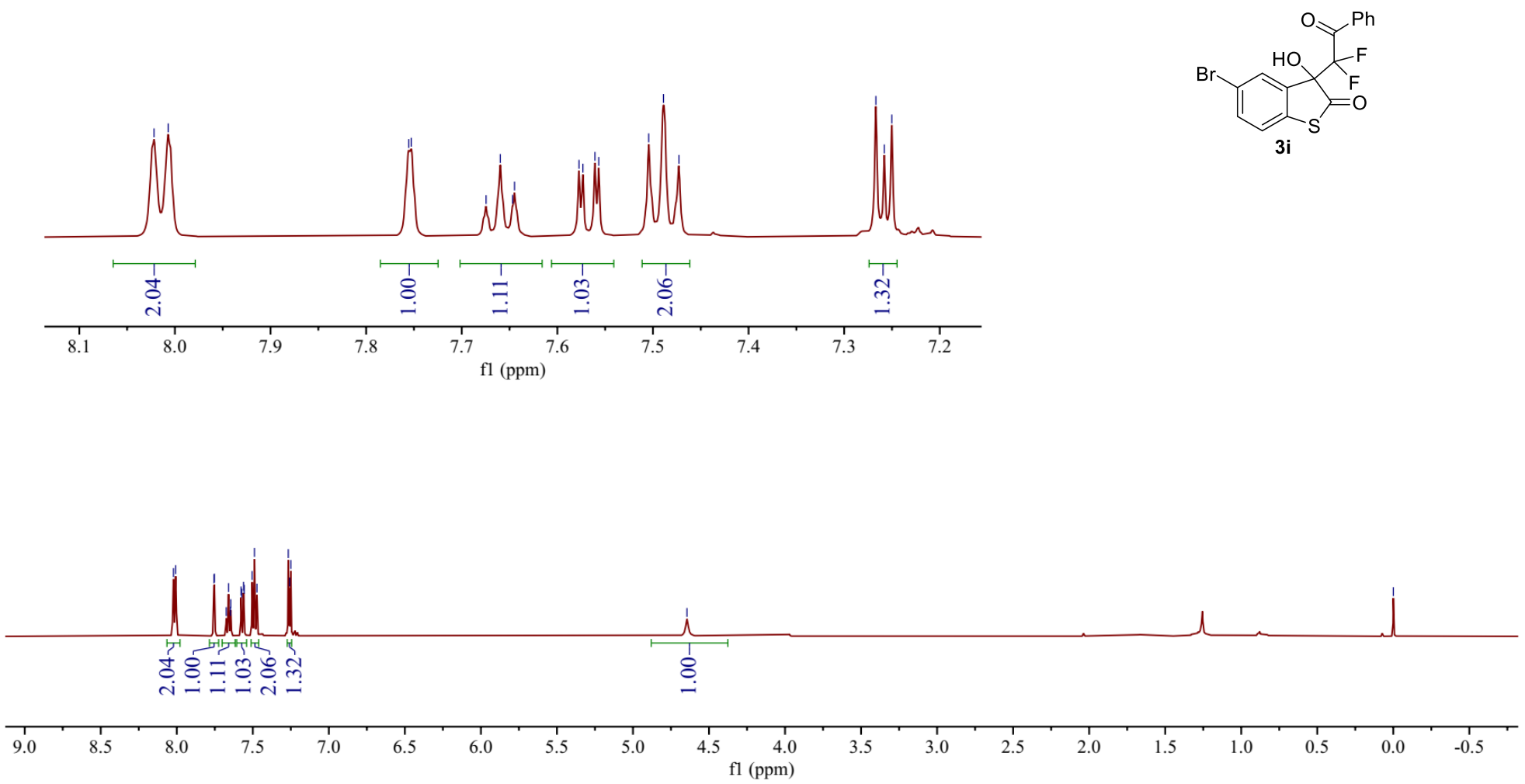

${ }^{1} \mathrm{H}$ NMR spectra of $\mathbf{3 i}$ in $\mathrm{CDCl}_{3}(400 \mathrm{MHz})$ 


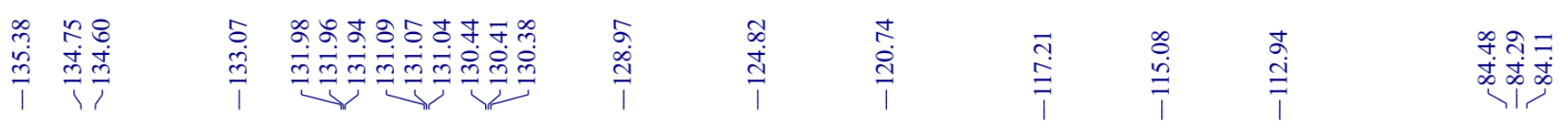
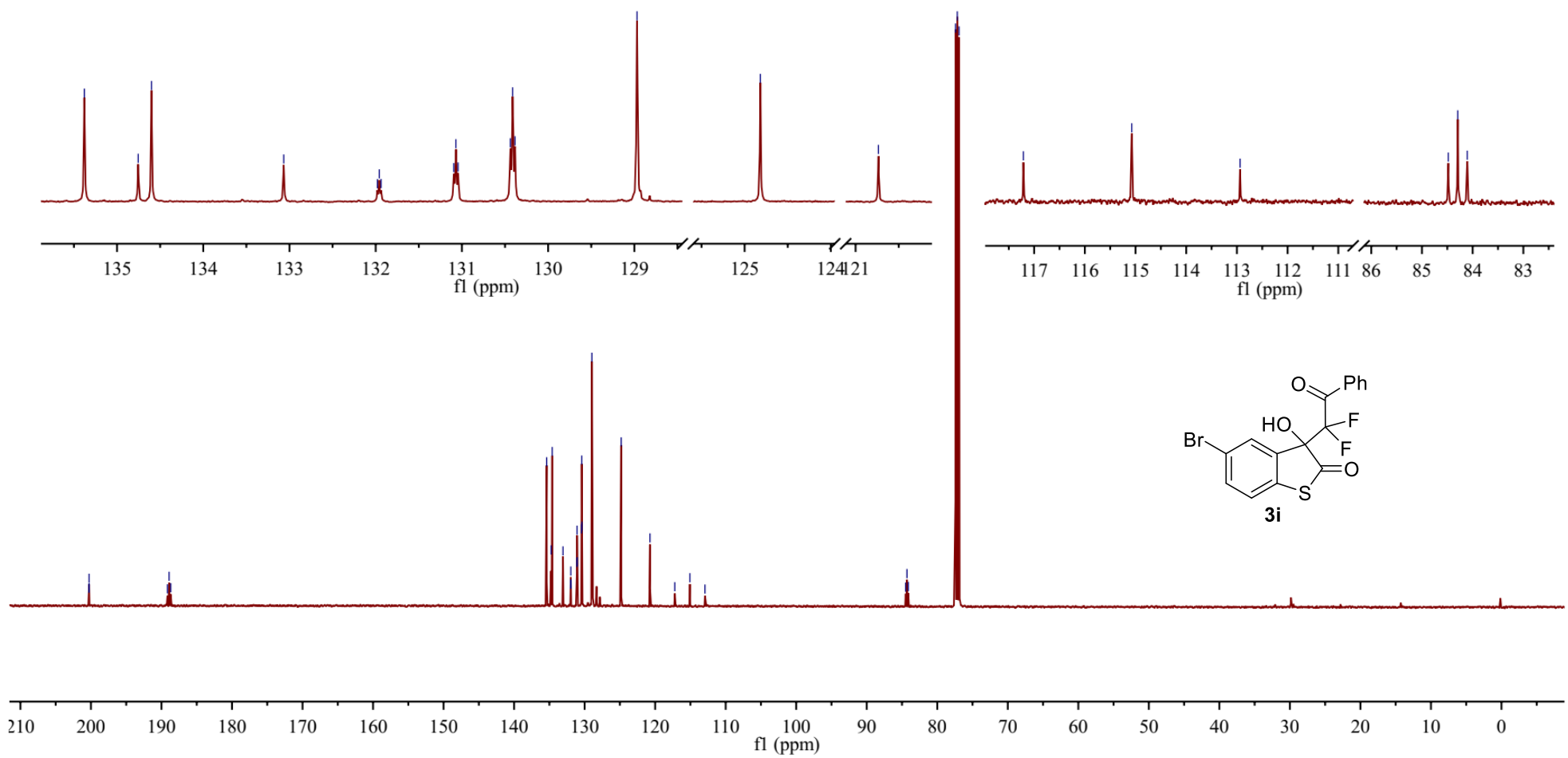

${ }^{13} \mathrm{C}\left\{{ }^{1} \mathrm{H}\right\}$ NMR spectra of $\mathbf{3 i}$ in $\mathrm{CDCl}_{3}(100 \mathrm{MHz})$ 


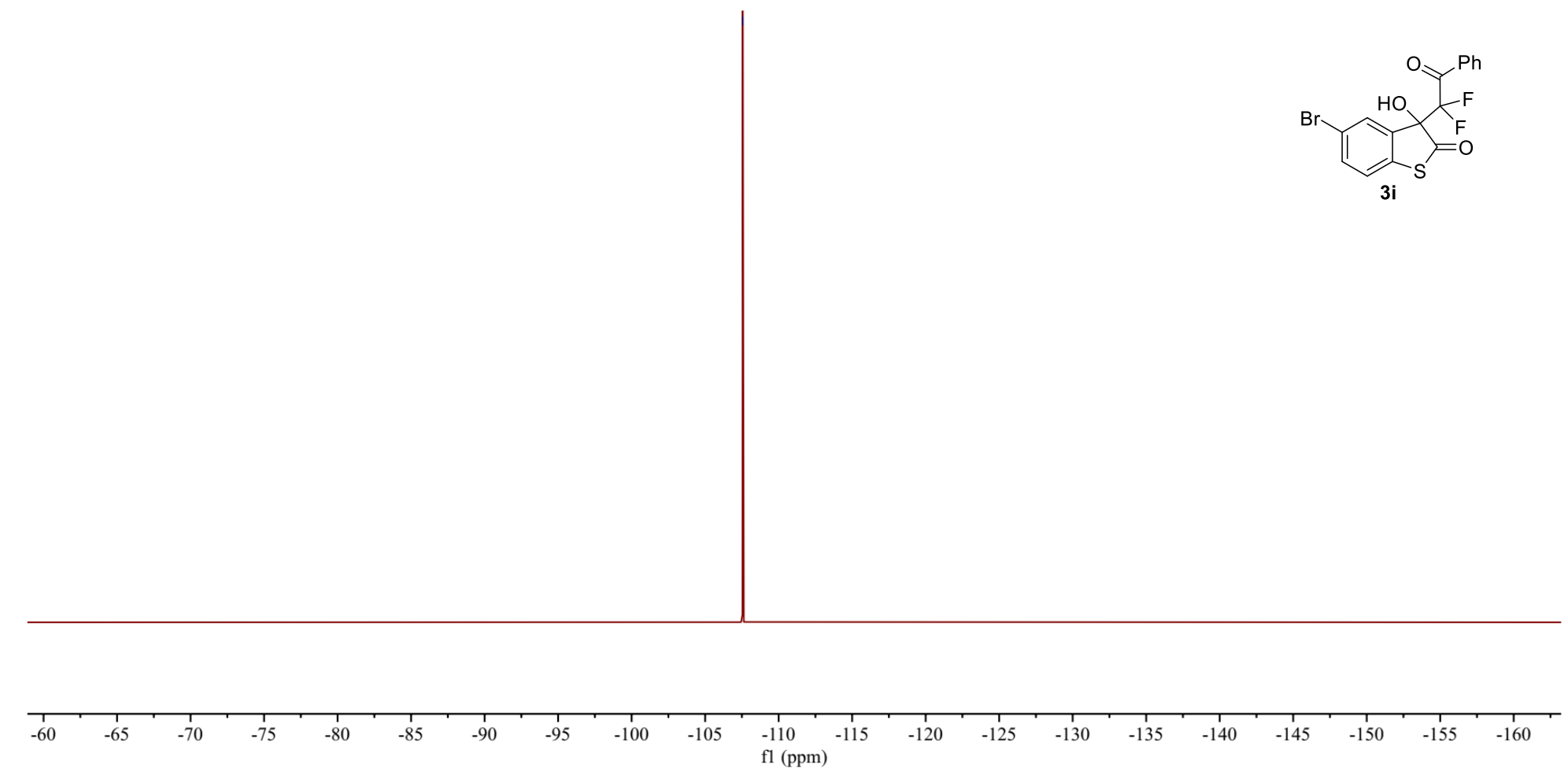

${ }^{19} \mathrm{~F}$ NMR spectra of $\mathbf{3 i}$ in $\mathrm{CDCl}_{3}(376 \mathrm{MHz})$ 

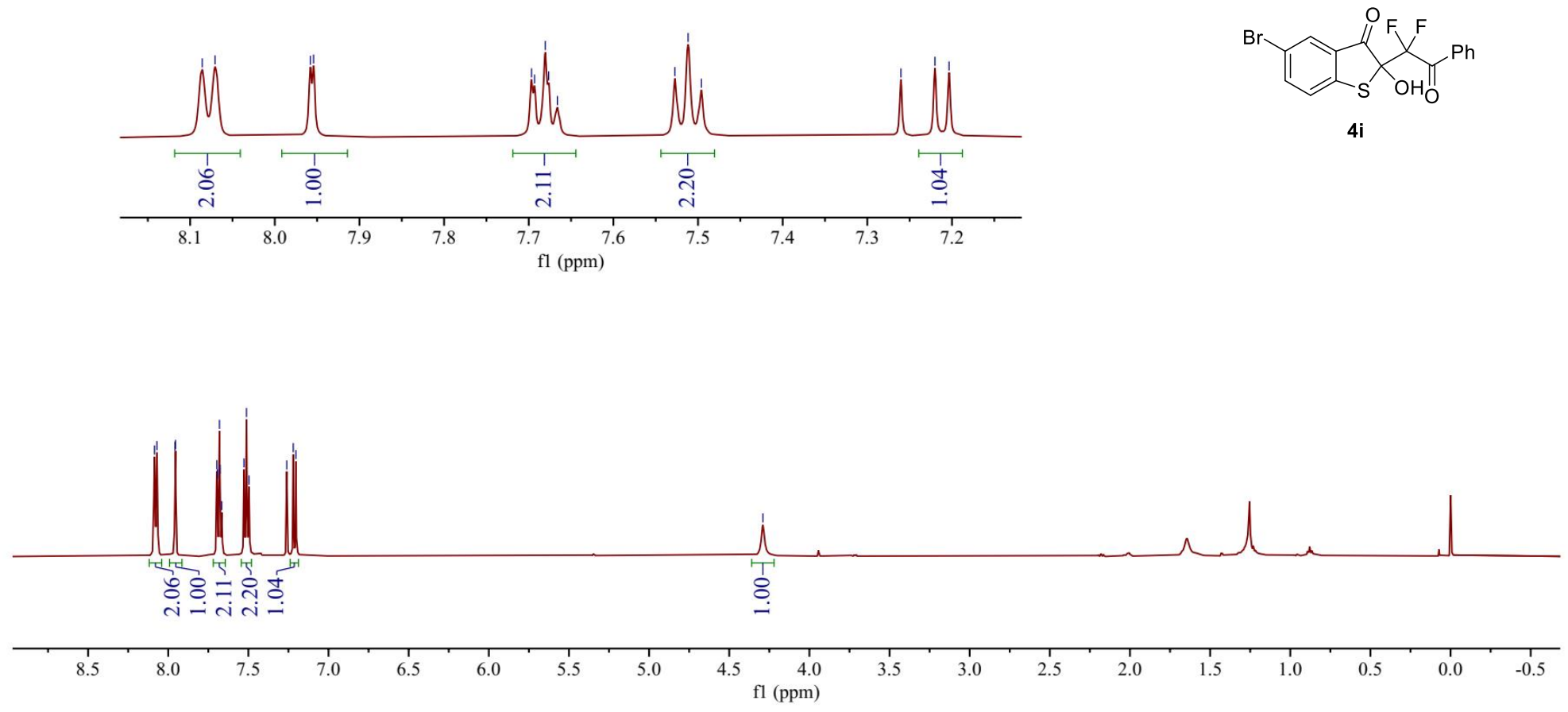

${ }^{1} \mathrm{H}$ NMR spectra of $4 \mathbf{i}$ in $\mathrm{CDCl}_{3}(500 \mathrm{MHz})$ 

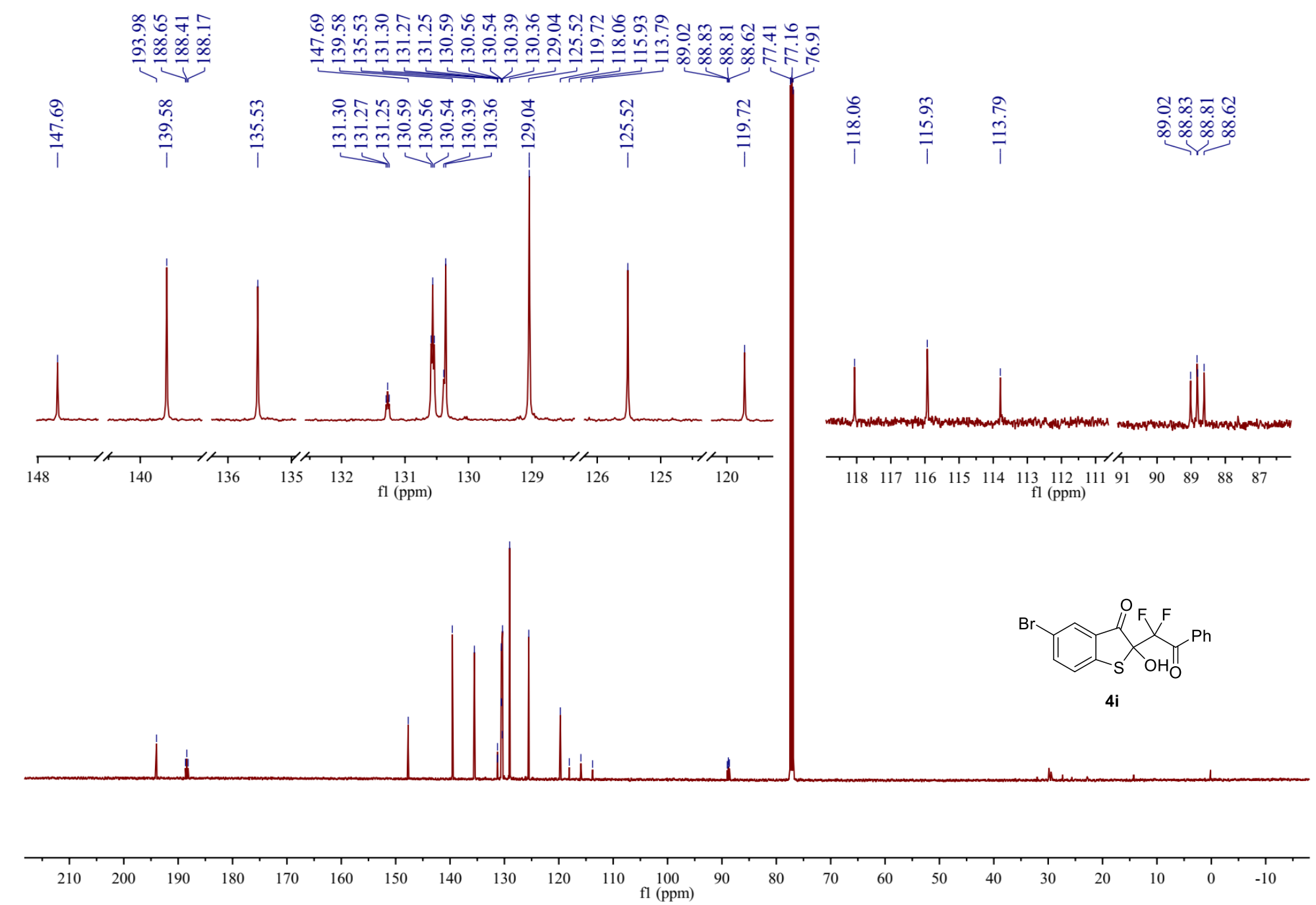

${ }^{13} \mathrm{C}\left\{{ }^{1} \mathrm{H}\right\}$ NMR spectra of $4 \mathbf{i}$ in $\mathrm{CDCl}_{3}(125 \mathrm{MHz})$ 


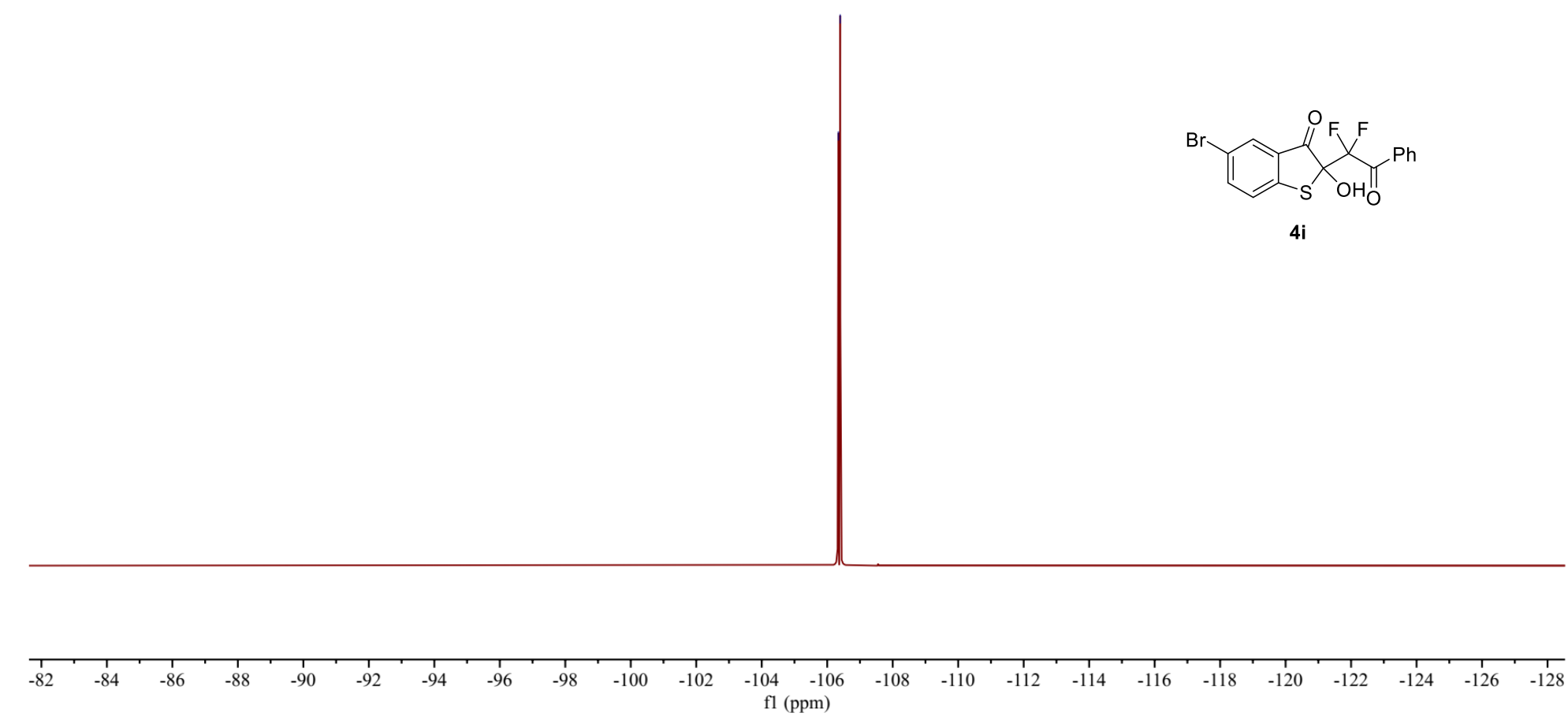

${ }^{19} \mathrm{~F}$ NMR spectra of $4 \mathbf{i}$ in $\mathrm{CDCl}_{3}(376 \mathrm{MHz})$ 


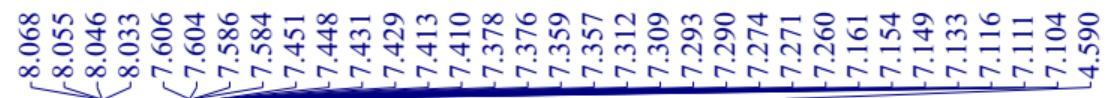

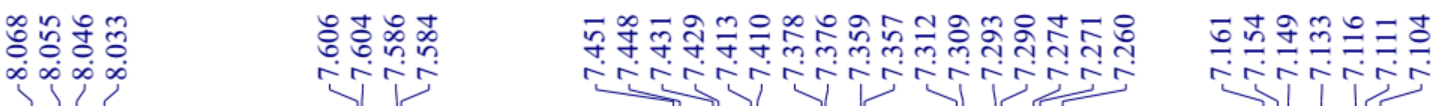
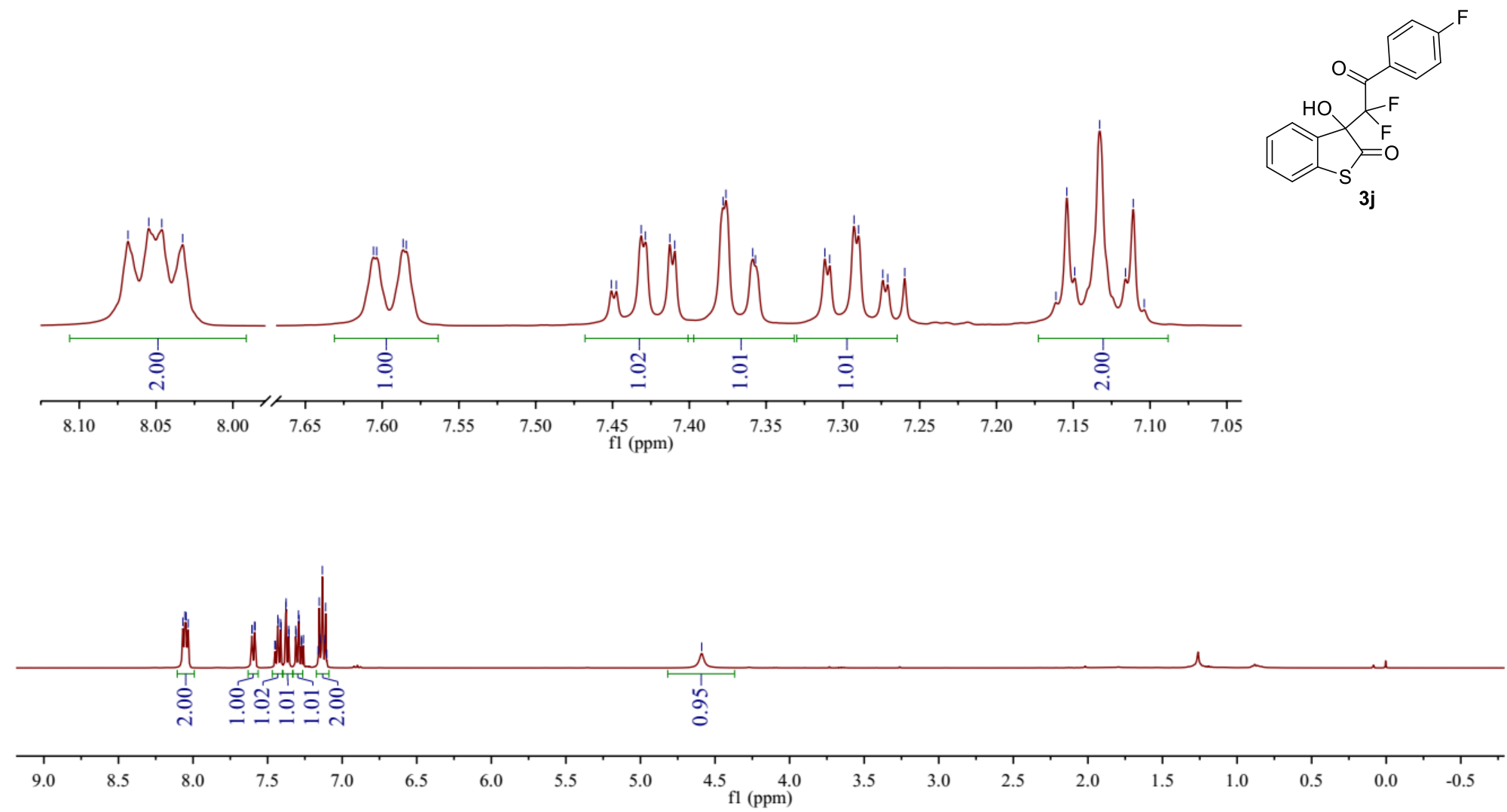

${ }^{1} \mathrm{H}$ NMR spectra of $\mathbf{3 j}$ in $\mathrm{CDCl}_{3}(400 \mathrm{MHz})$ 

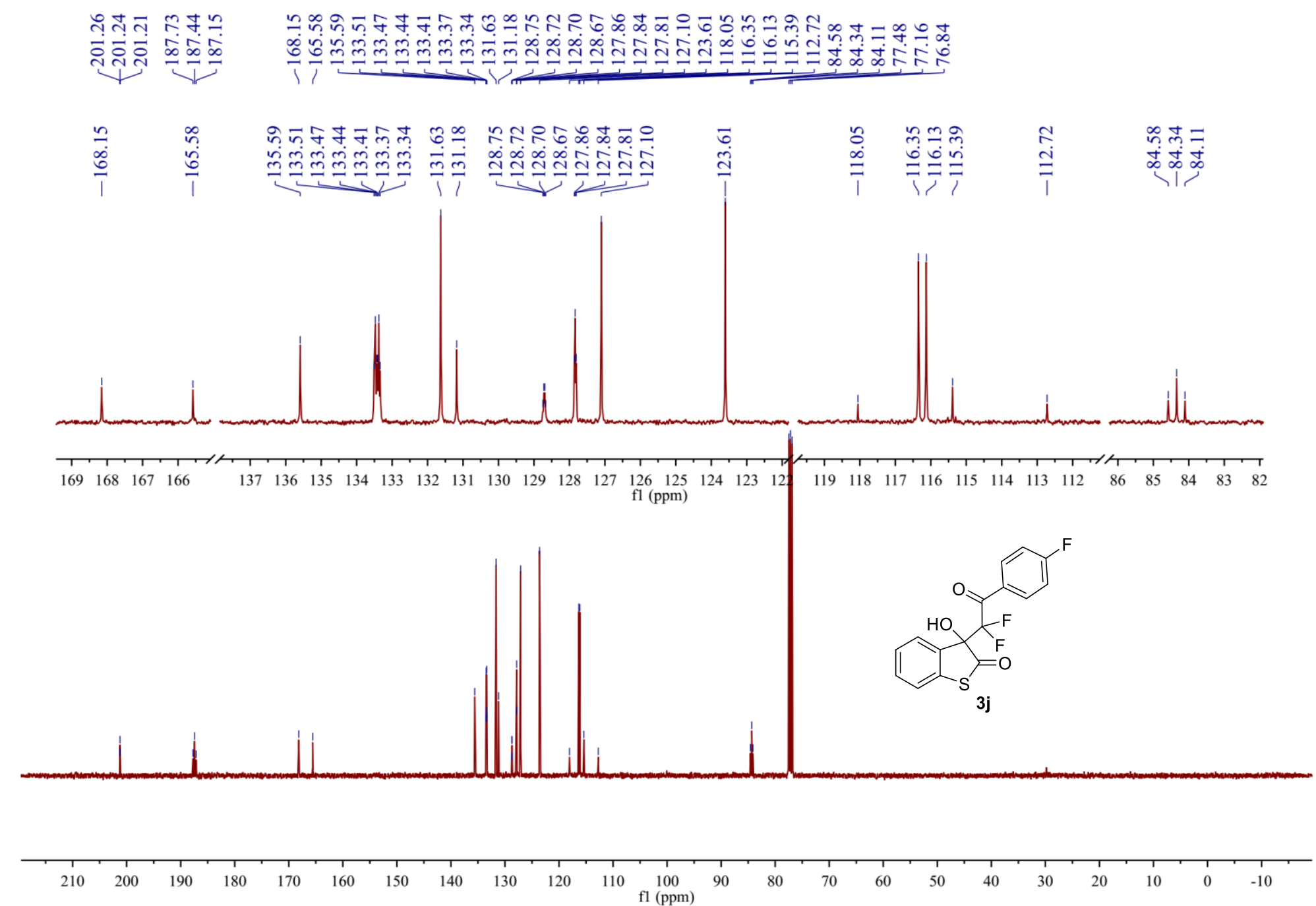

${ }^{13} \mathrm{C}\left\{{ }^{1} \mathrm{H}\right\}$ NMR spectra of $\mathbf{3 j}$ in $\mathrm{CDCl}_{3}(100 \mathrm{MHz})$ 

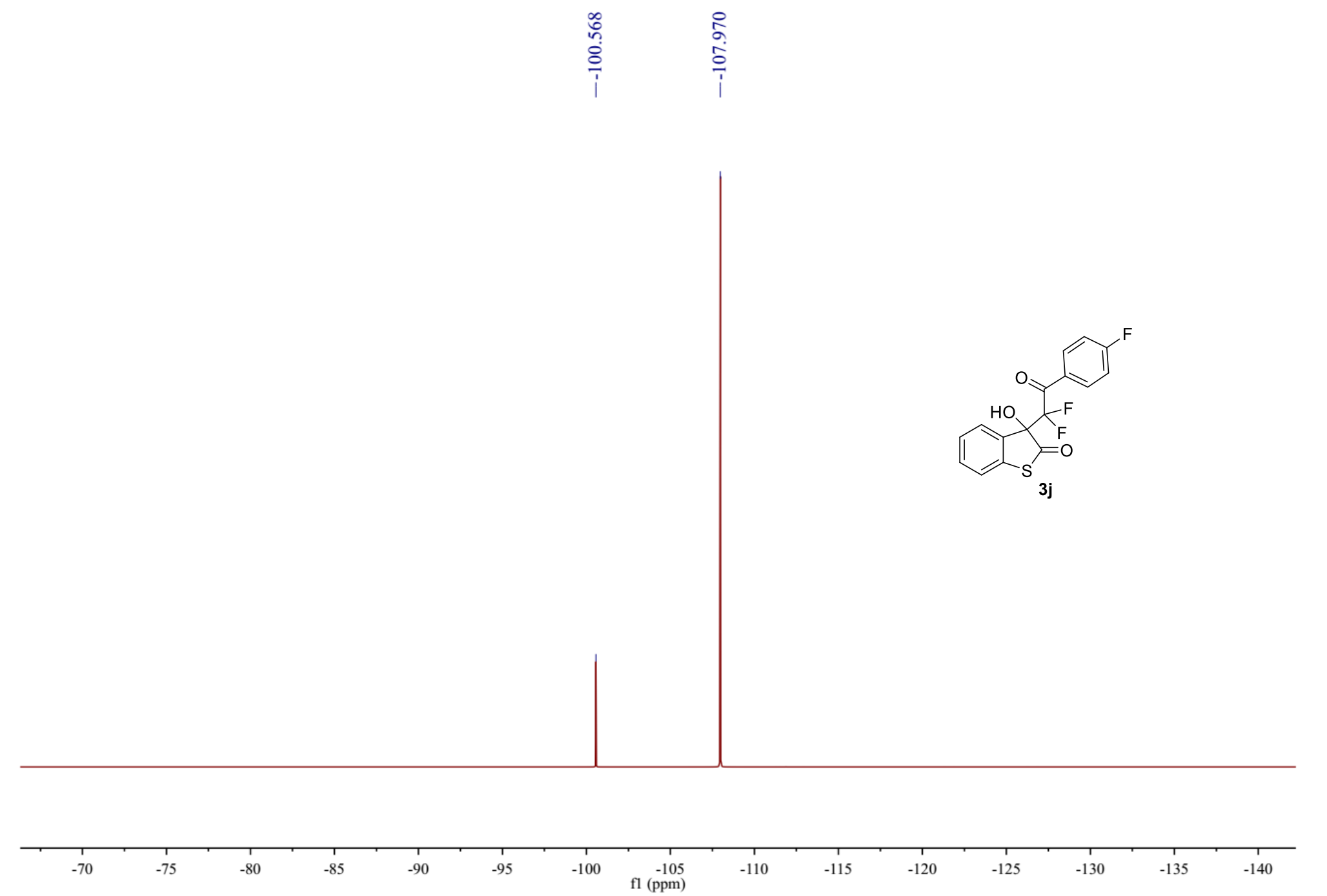

${ }^{19} \mathrm{~F}$ NMR spectra of $\mathbf{3 j}$ in $\mathrm{CDCl}_{3}(376 \mathrm{MHz})$ 


\begin{tabular}{|c|c|c|c|}
\hline 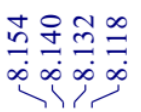 & 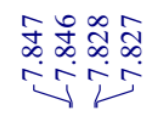 & 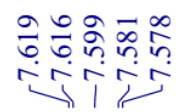 & 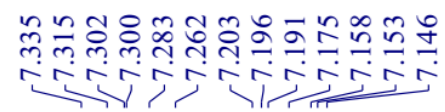 \\
\hline
\end{tabular}

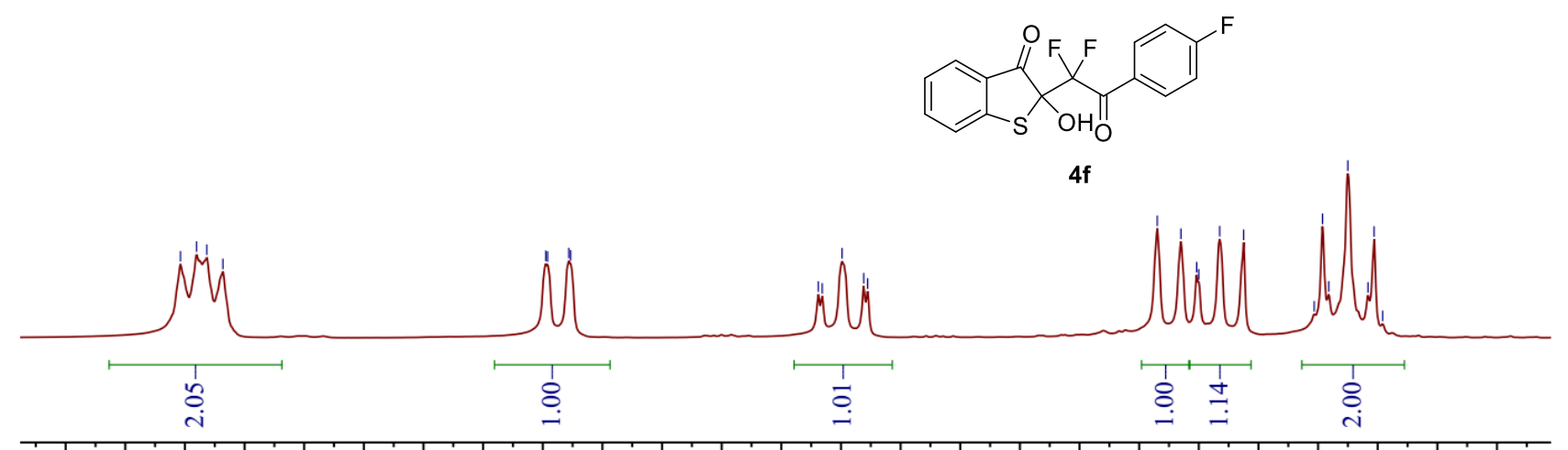

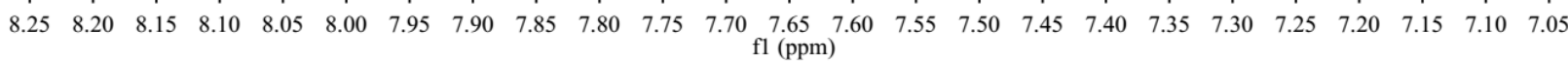

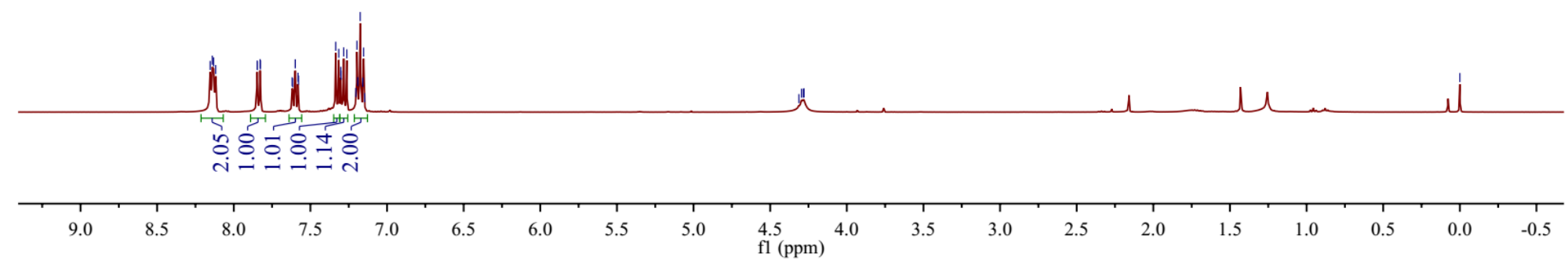

${ }^{1} \mathrm{H}$ NMR spectra of $\mathbf{4} \mathbf{j}$ in $\mathrm{CDCl}_{3}(400 \mathrm{MHz})$ 

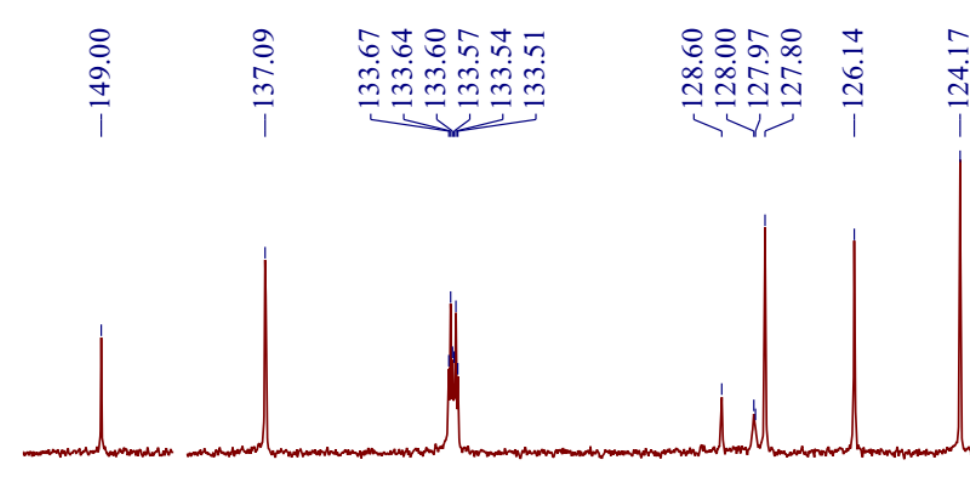

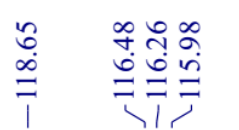

$\stackrel{m}{\stackrel{m}{7}}$

กิำ तิธ $\underbrace{\infty}$
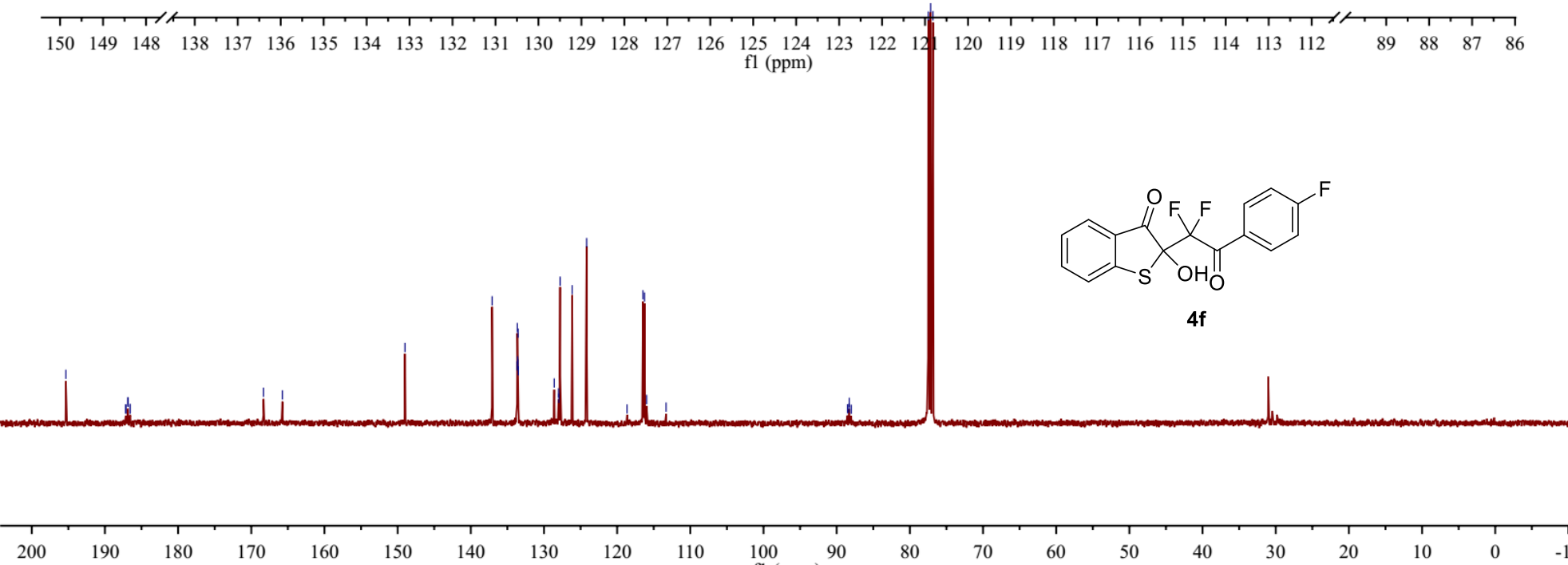

$140 \quad 130$

120

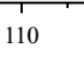

100
$\mathrm{fl}(\mathrm{ppm})$ 90
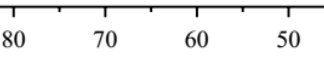

40

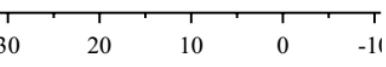

${ }^{13} \mathrm{C}\left\{{ }^{1} \mathrm{H}\right\}$ NMR spectra of $\mathbf{4} \mathbf{j}$ in $\mathrm{CDCl}_{3}(100 \mathrm{MHz})$ 

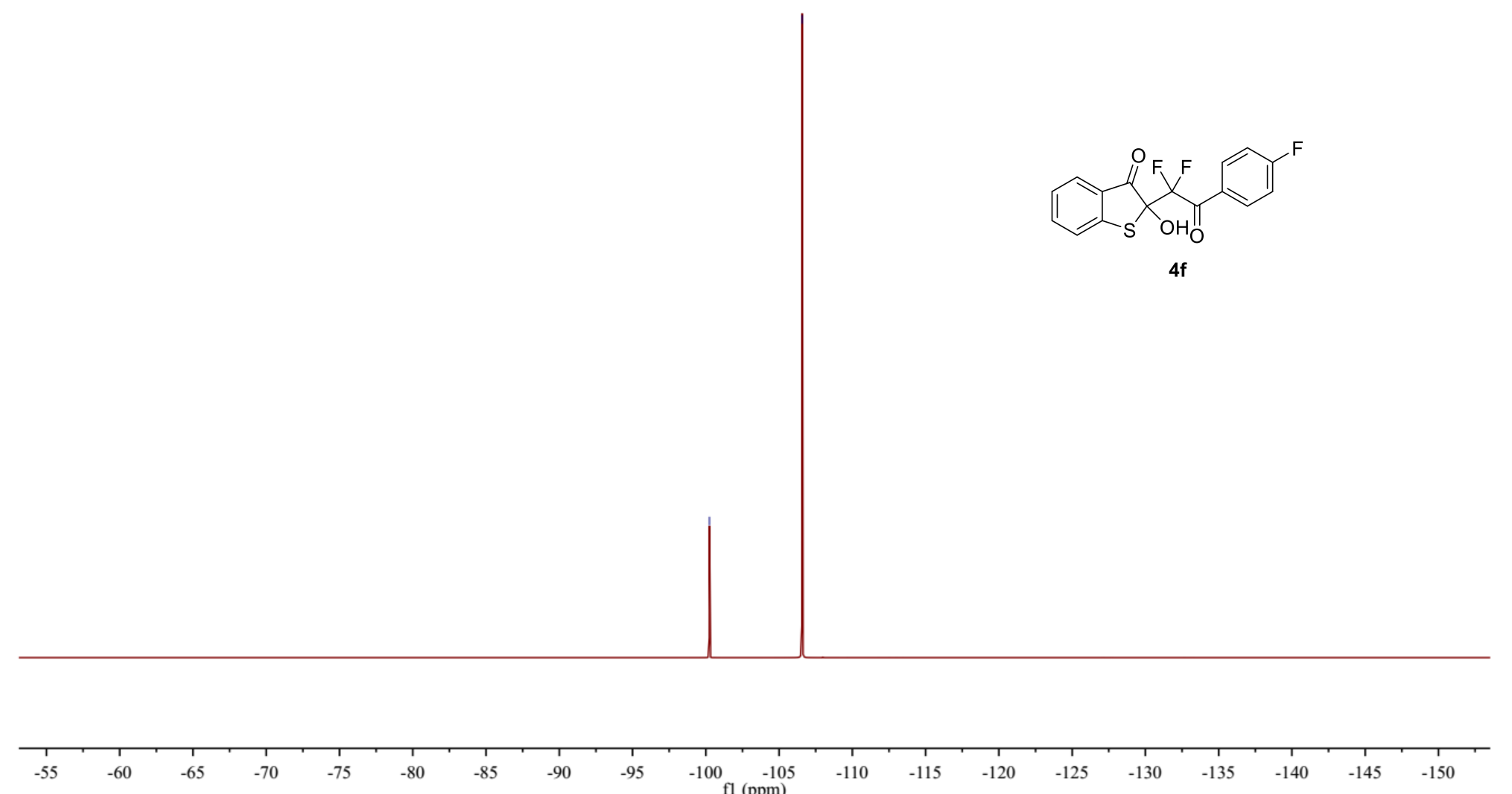

${ }^{19} \mathrm{~F}$ NMR spectra of $\mathbf{4 j}$ in $\mathrm{CDCl}_{3}(376 \mathrm{MHz})$ 


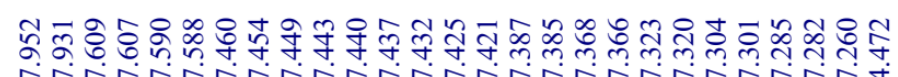

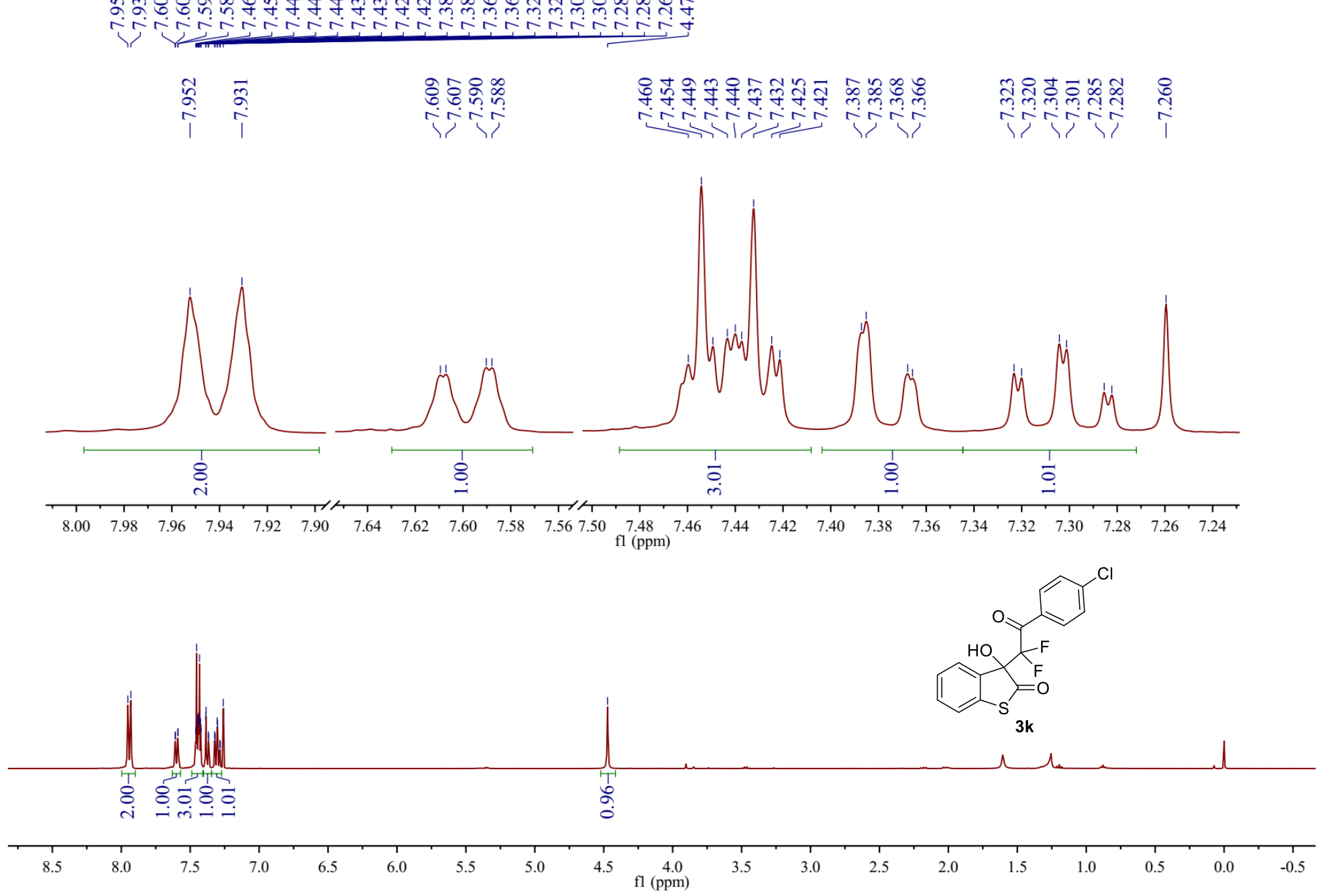

${ }^{1} \mathrm{H}$ NMR spectra of $\mathbf{3 k}$ in $\mathrm{CDCl}_{3}(400 \mathrm{MHz})$ 

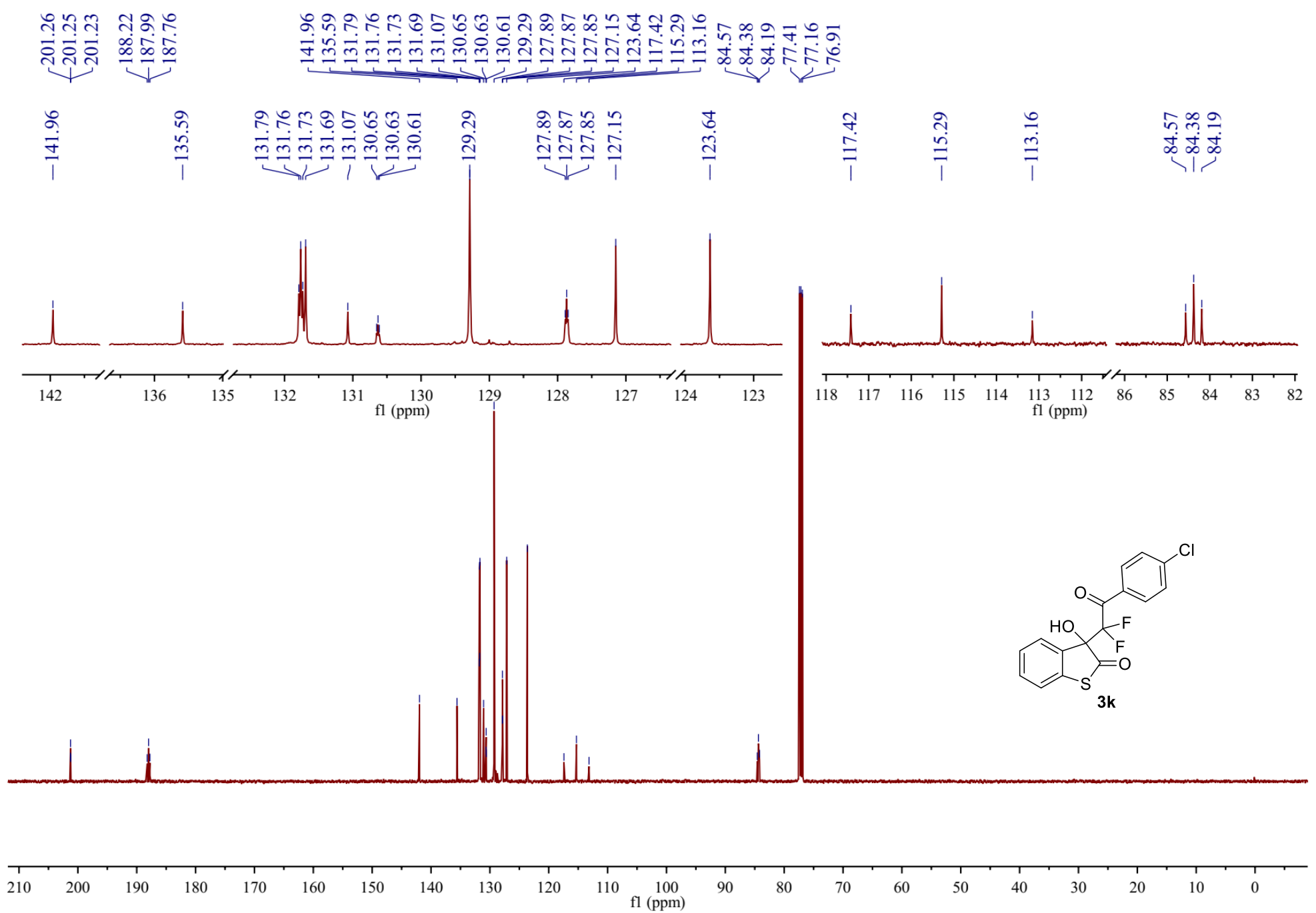

${ }^{13} \mathrm{C}\left\{{ }^{1} \mathrm{H}\right\}$ NMR spectra of $\mathbf{3 k}$ in $\mathrm{CDCl}_{3}(125 \mathrm{MHz})$ 


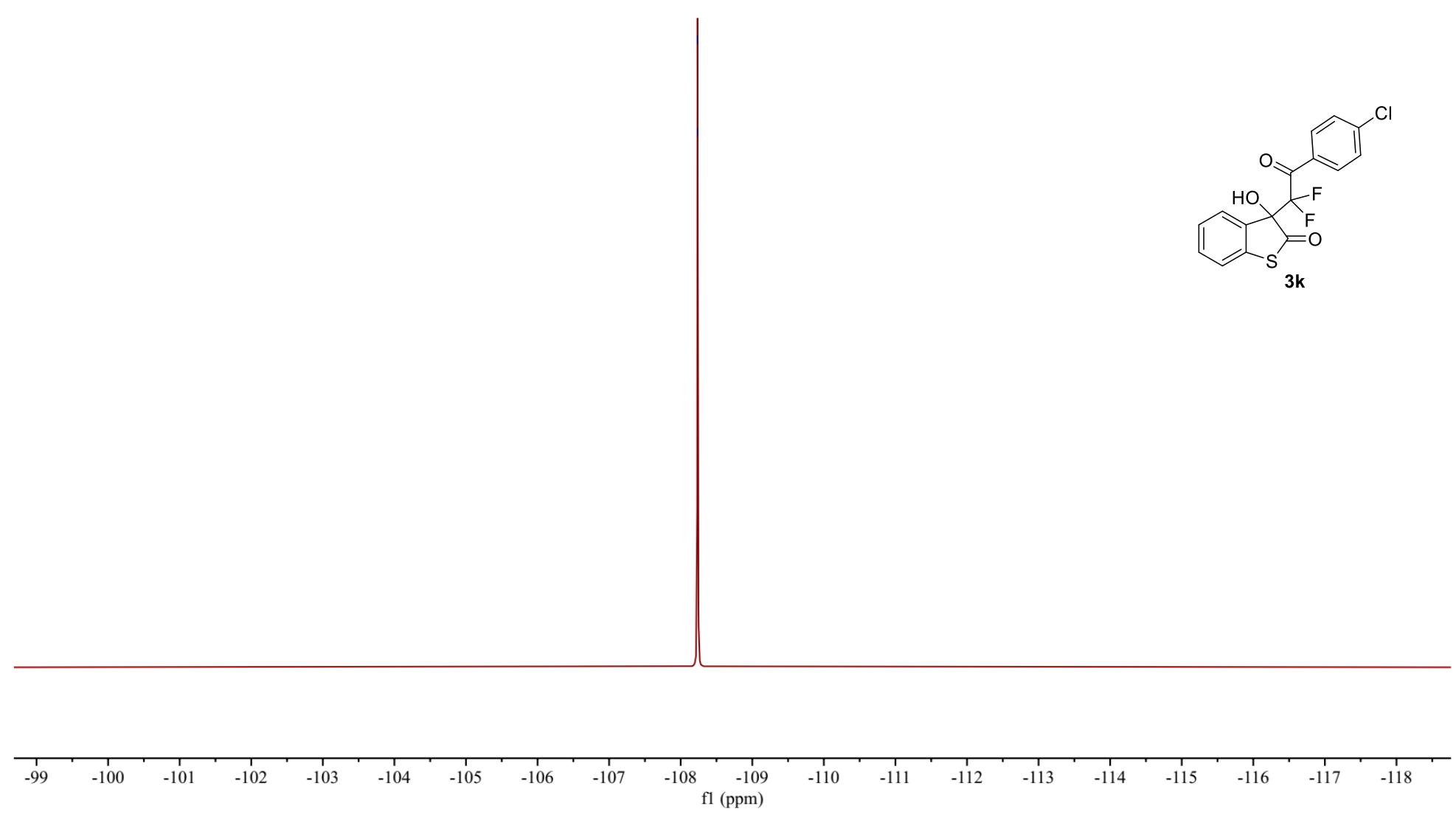

${ }^{19} \mathrm{~F}$ NMR spectra of $\mathbf{3 k}$ in $\mathrm{CDCl}_{3}(376 \mathrm{MHz})$ 

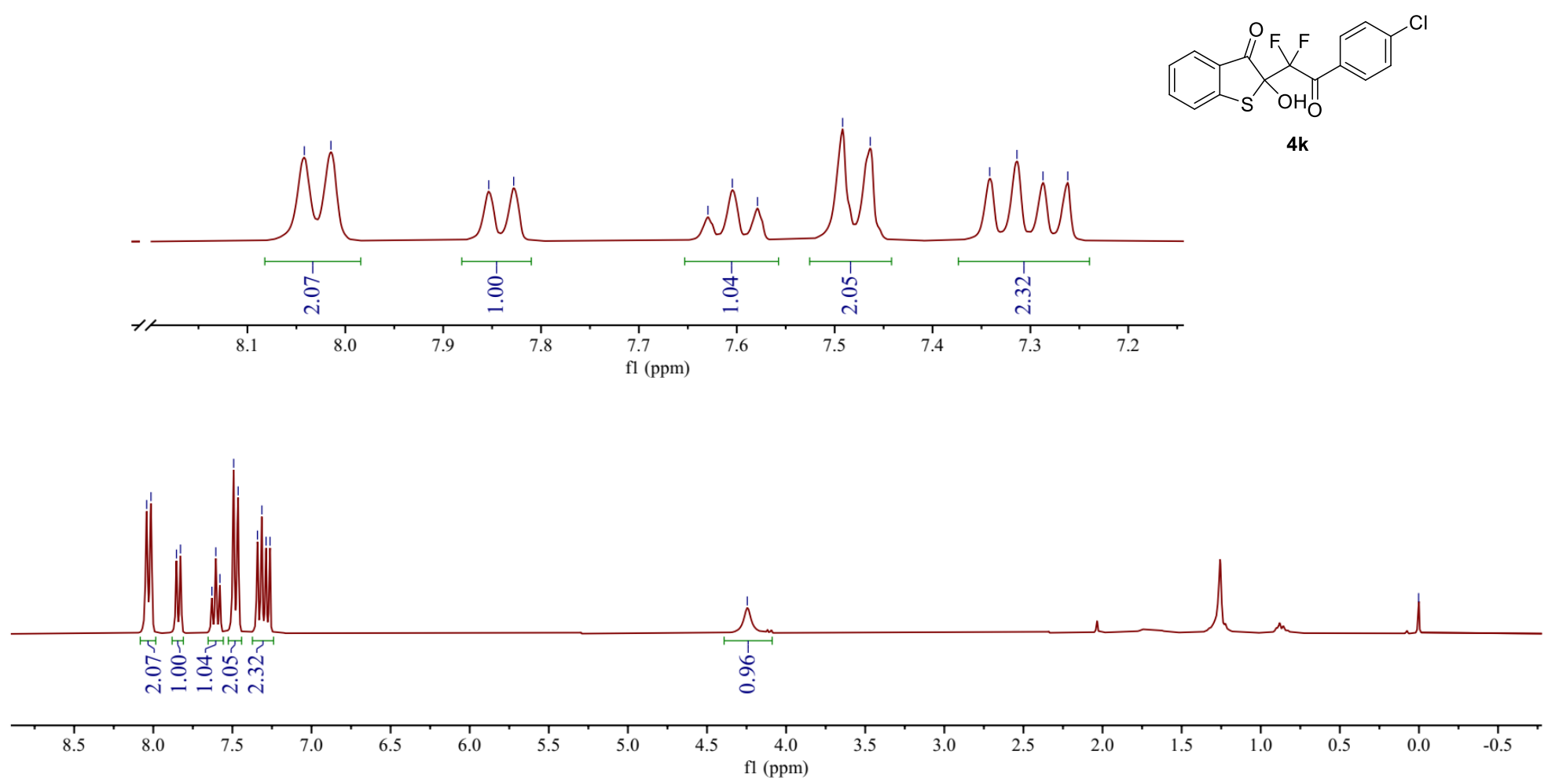

${ }^{1} \mathrm{H}$ NMR spectra of $\mathbf{4 k}$ in $\mathrm{CDCl}_{3}(300 \mathrm{MHz})$ 

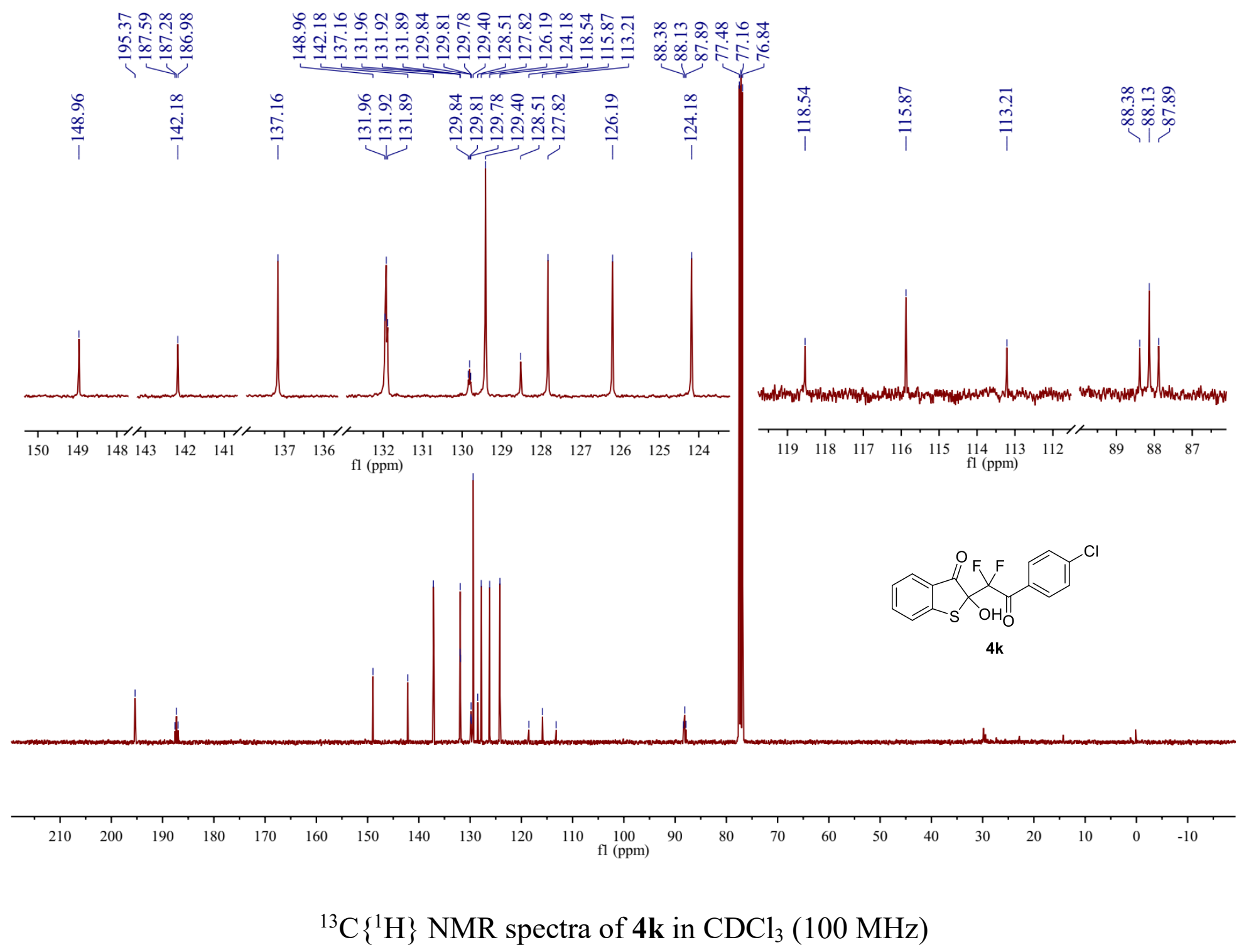


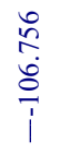

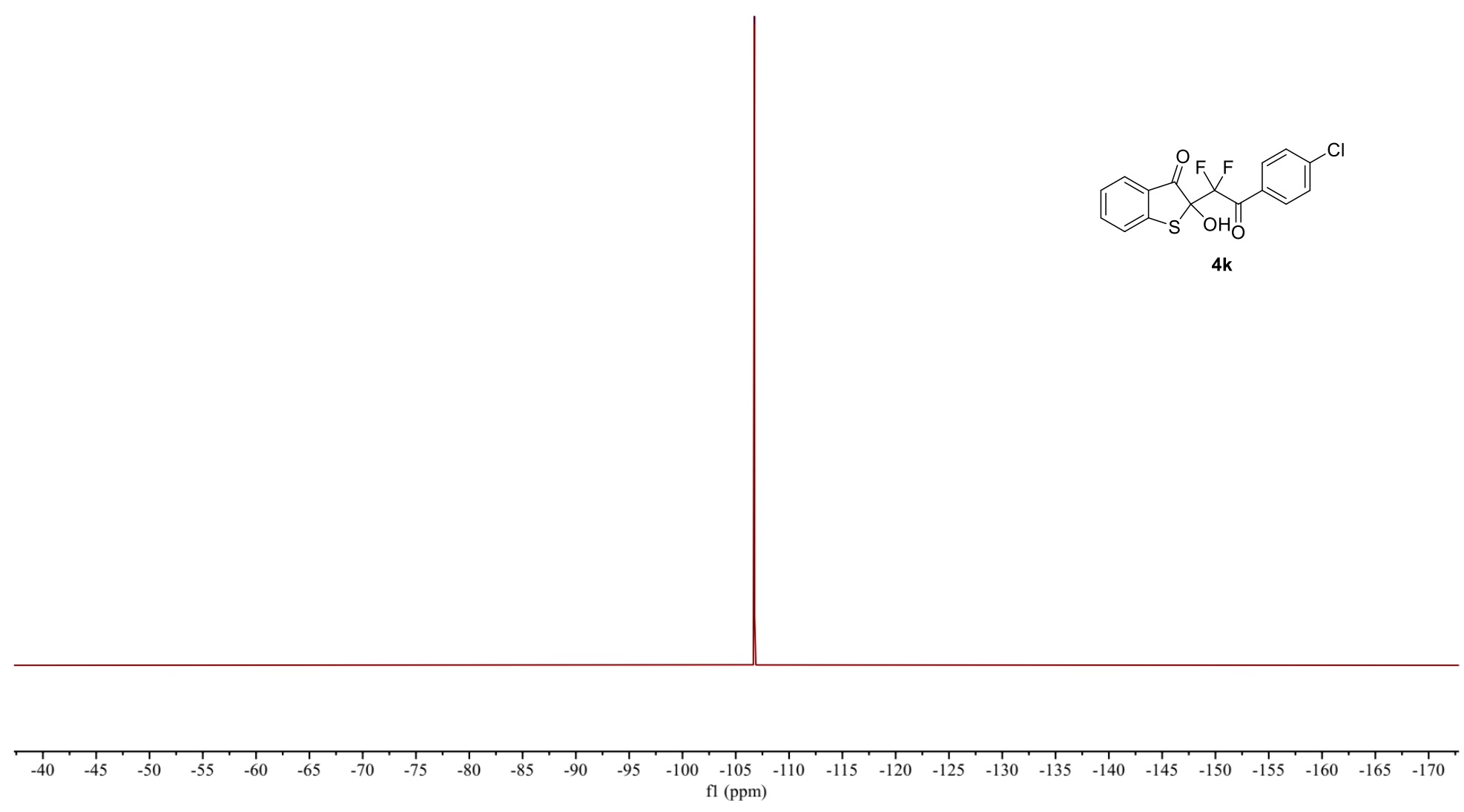

${ }^{19} \mathrm{~F}$ NMR spectra of $\mathbf{4 k}$ in $\mathrm{CDCl}_{3}(376 \mathrm{MHz})$ 


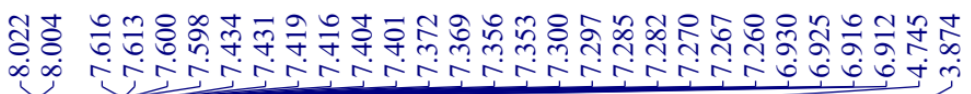
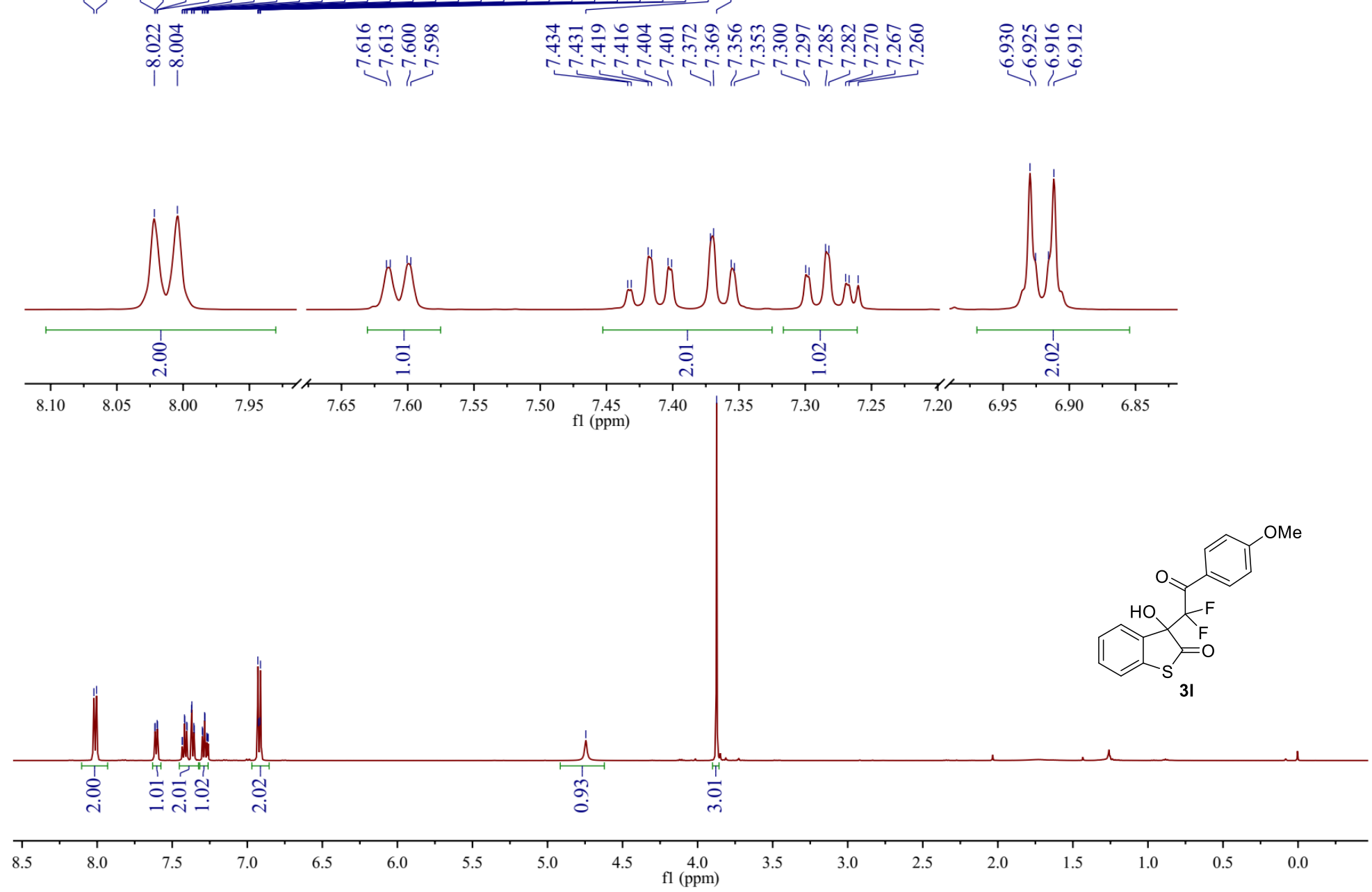

${ }^{1} \mathrm{H}$ NMR spectra of $\mathbf{3 l}$ in $\mathrm{CDCl}_{3}(500 \mathrm{MHz})$ 

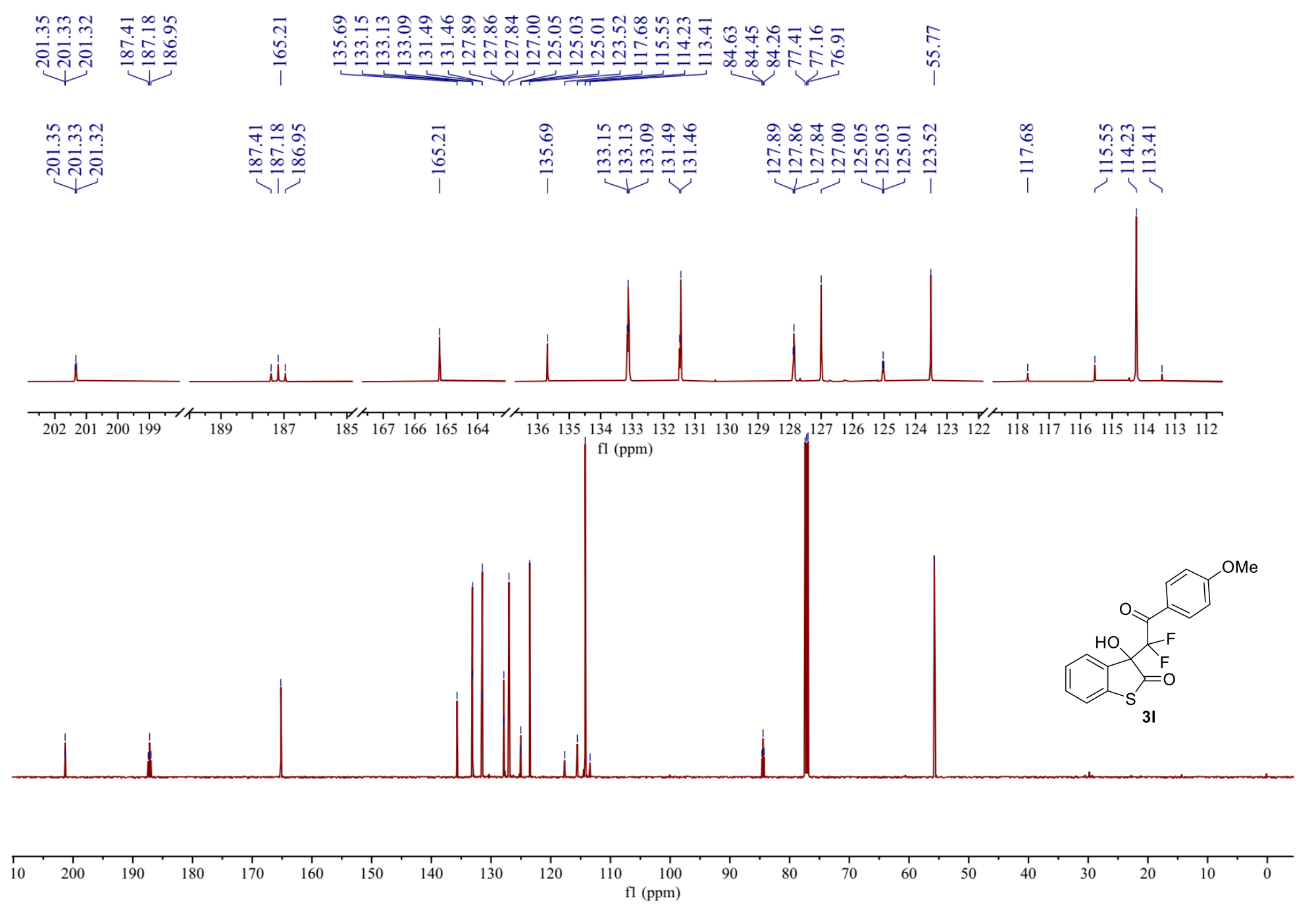

${ }^{13} \mathrm{C}\left\{{ }^{1} \mathrm{H}\right\}$ NMR spectra of $\mathbf{3 l}$ in $\mathrm{CDCl}_{3}(125 \mathrm{MHz})$ 


\section{午昌}

is

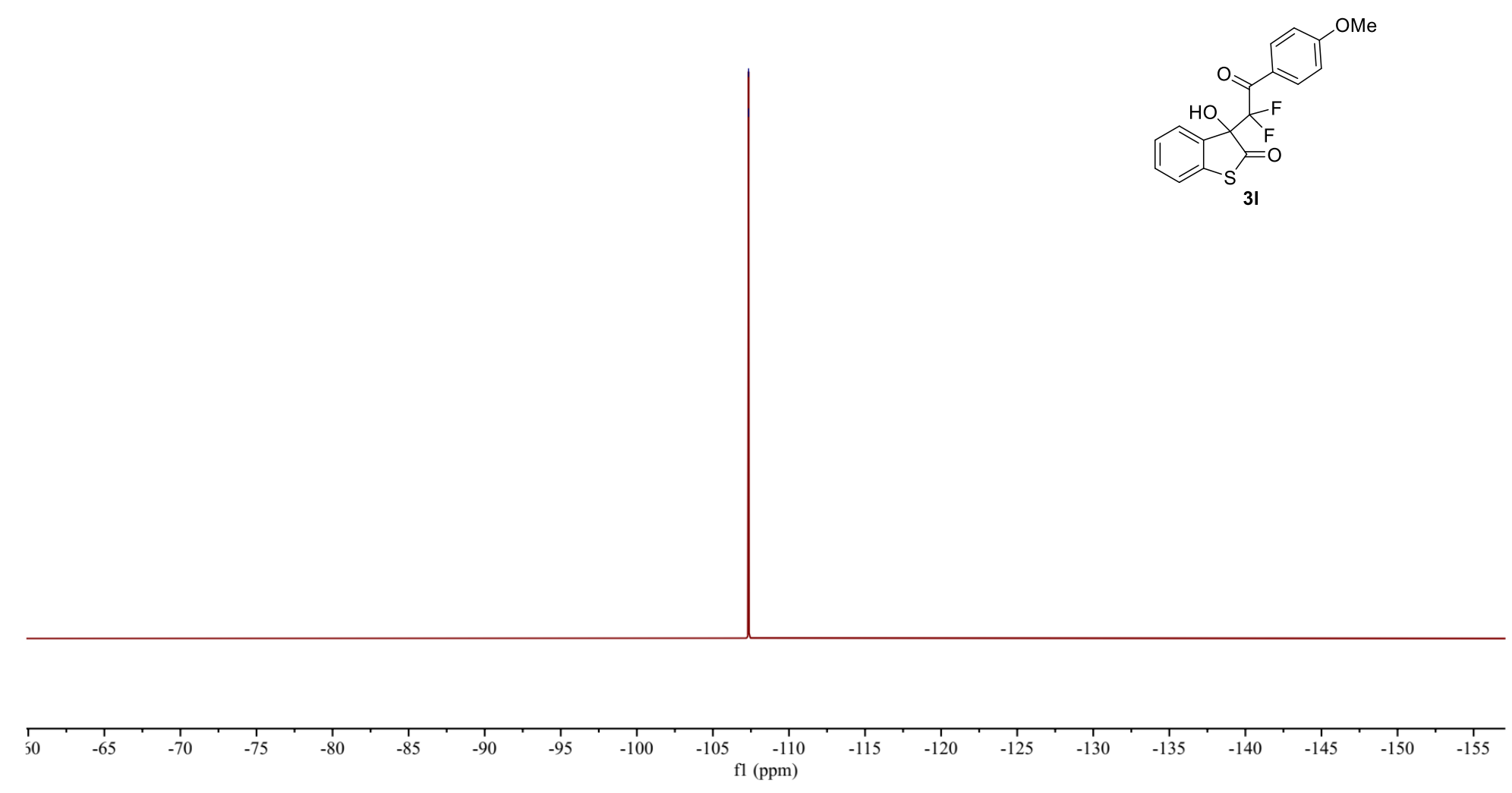

${ }^{19} \mathrm{~F}$ NMR spectra of $\mathbf{3 l}$ in $\mathrm{CDCl}_{3}(376 \mathrm{MHz})$ 


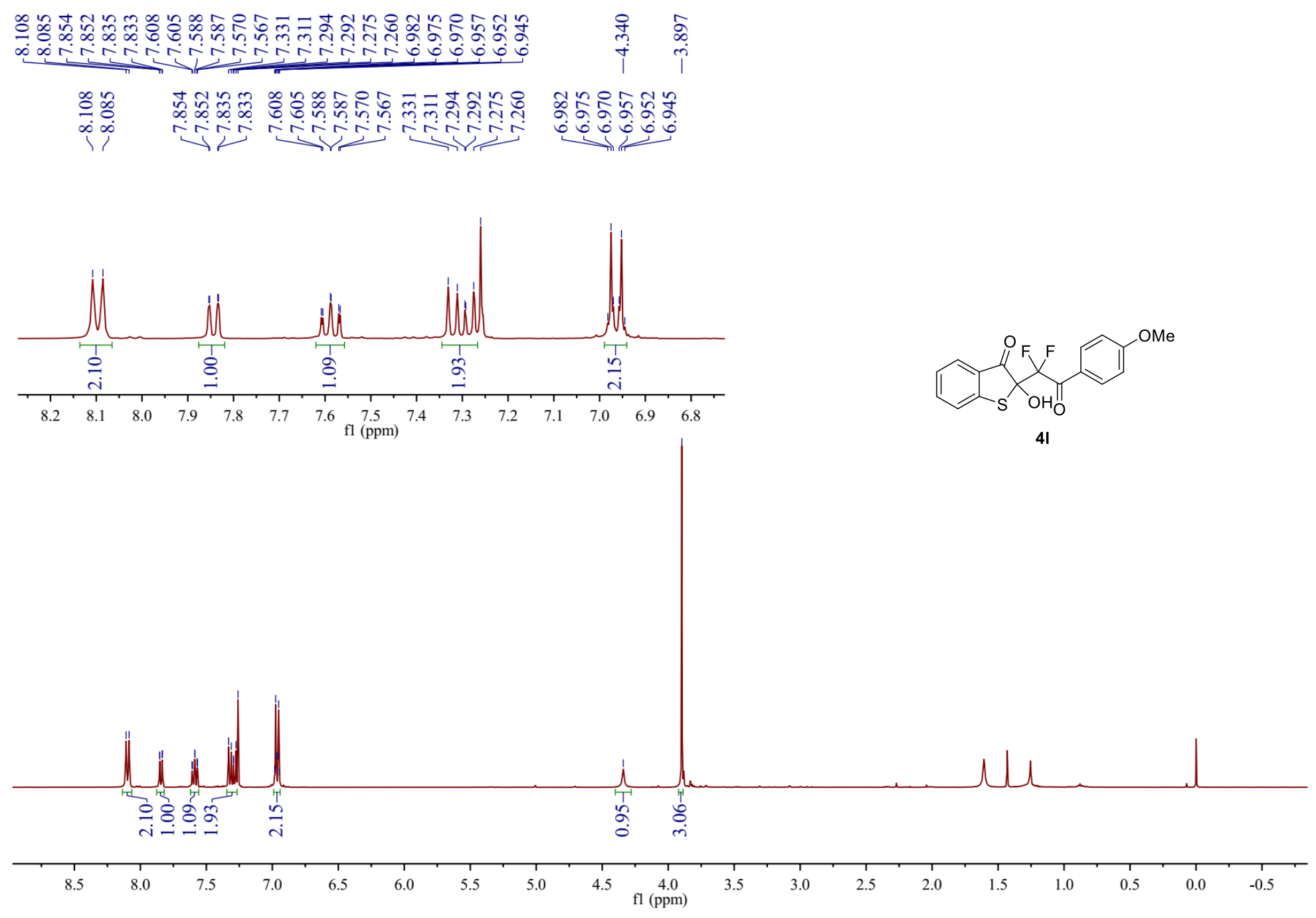

${ }^{1} \mathrm{H}$ NMR spectra of $4 \mathbf{l}$ in $\mathrm{CDCl}_{3}(400 \mathrm{MHz})$ 

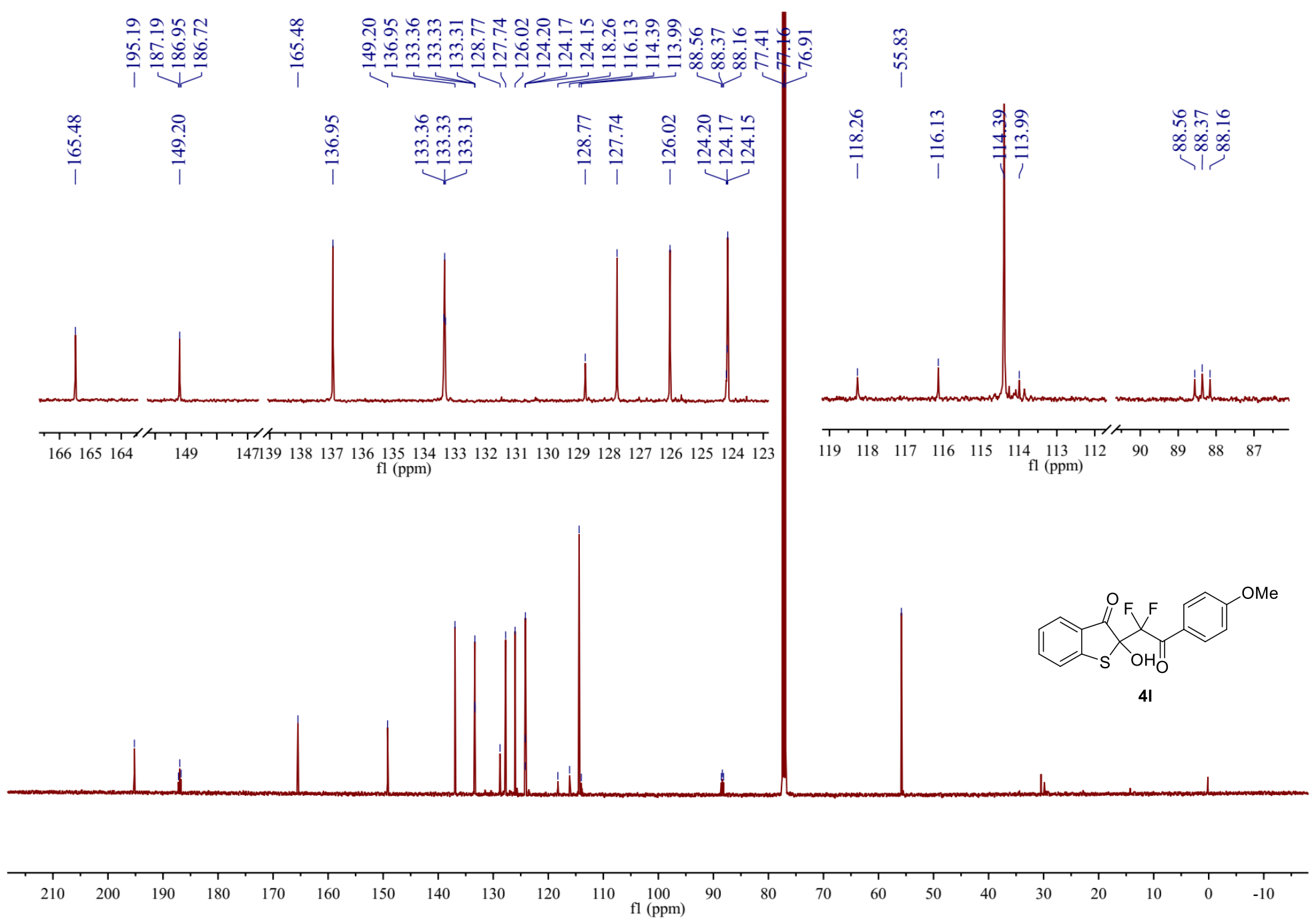

${ }^{13} \mathrm{C}\left\{{ }^{1} \mathrm{H}\right\}$ NMR spectra of $4 \mathrm{l}$ in $\mathrm{CDCl}_{3}(125 \mathrm{MHz})$ 

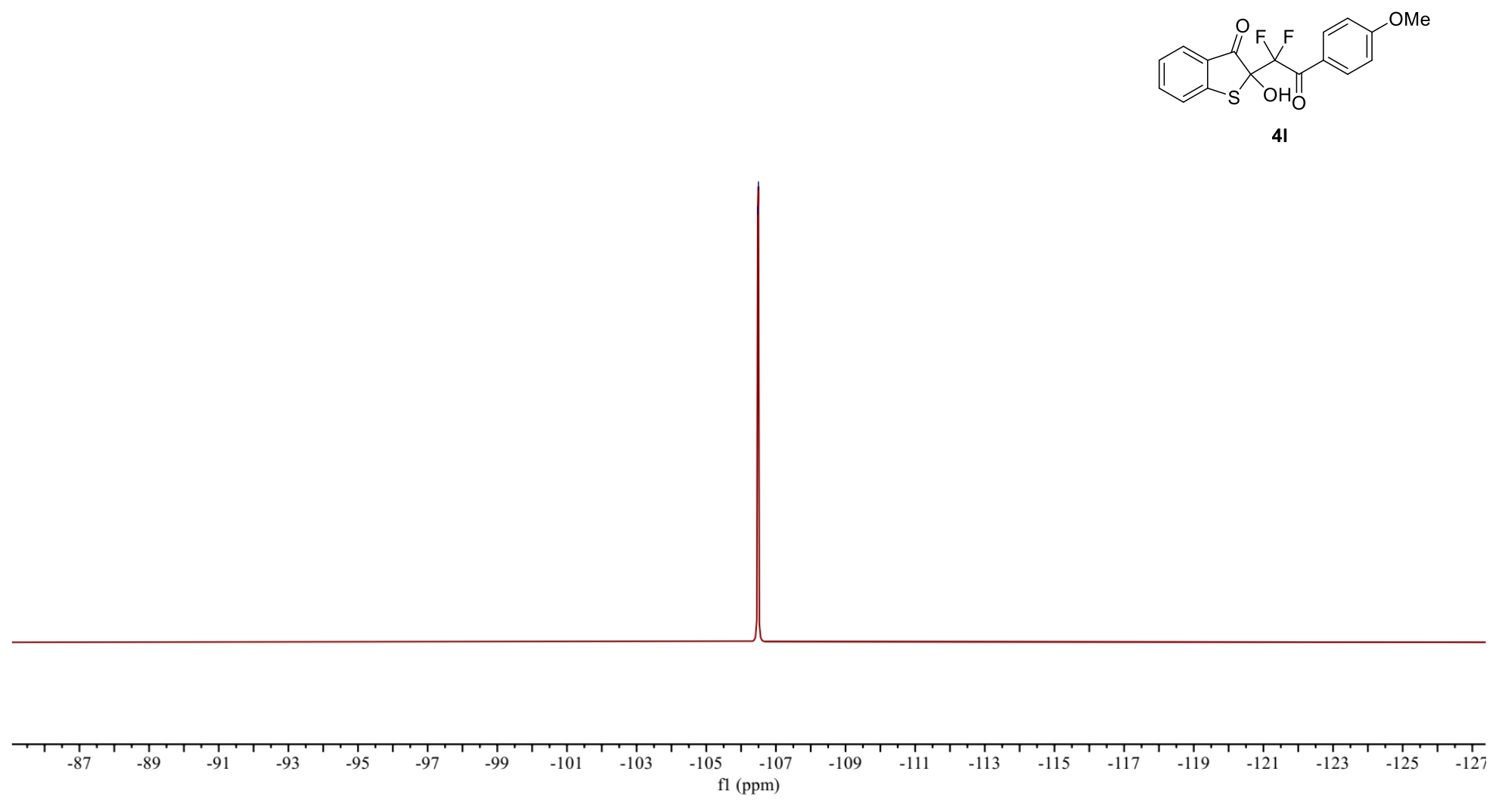

${ }^{19} \mathrm{~F}$ NMR spectra of $\mathbf{4 l}$ in $\mathrm{CDCl}_{3}(376 \mathrm{MHz})$ 
$\begin{array}{ll}\infty & \infty \\ 0 & \infty \\ 0 & \infty \\ & 1\end{array}$

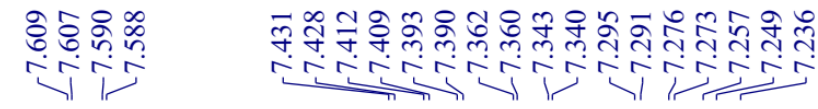
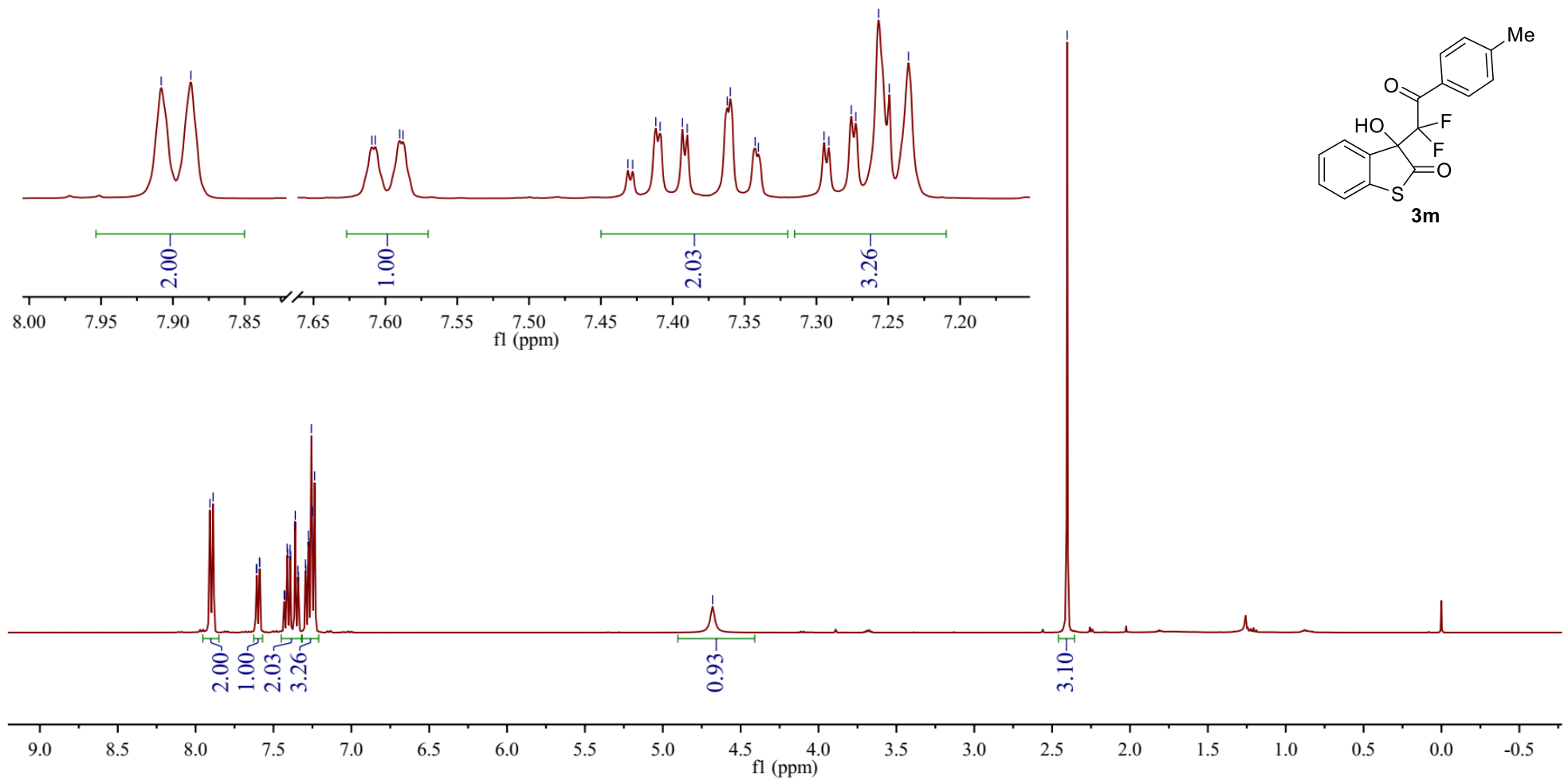

${ }^{1} \mathrm{H}$ NMR spectra of $\mathbf{3 m}$ in $\mathrm{CDCl}_{3}(400 \mathrm{MHz})$ 

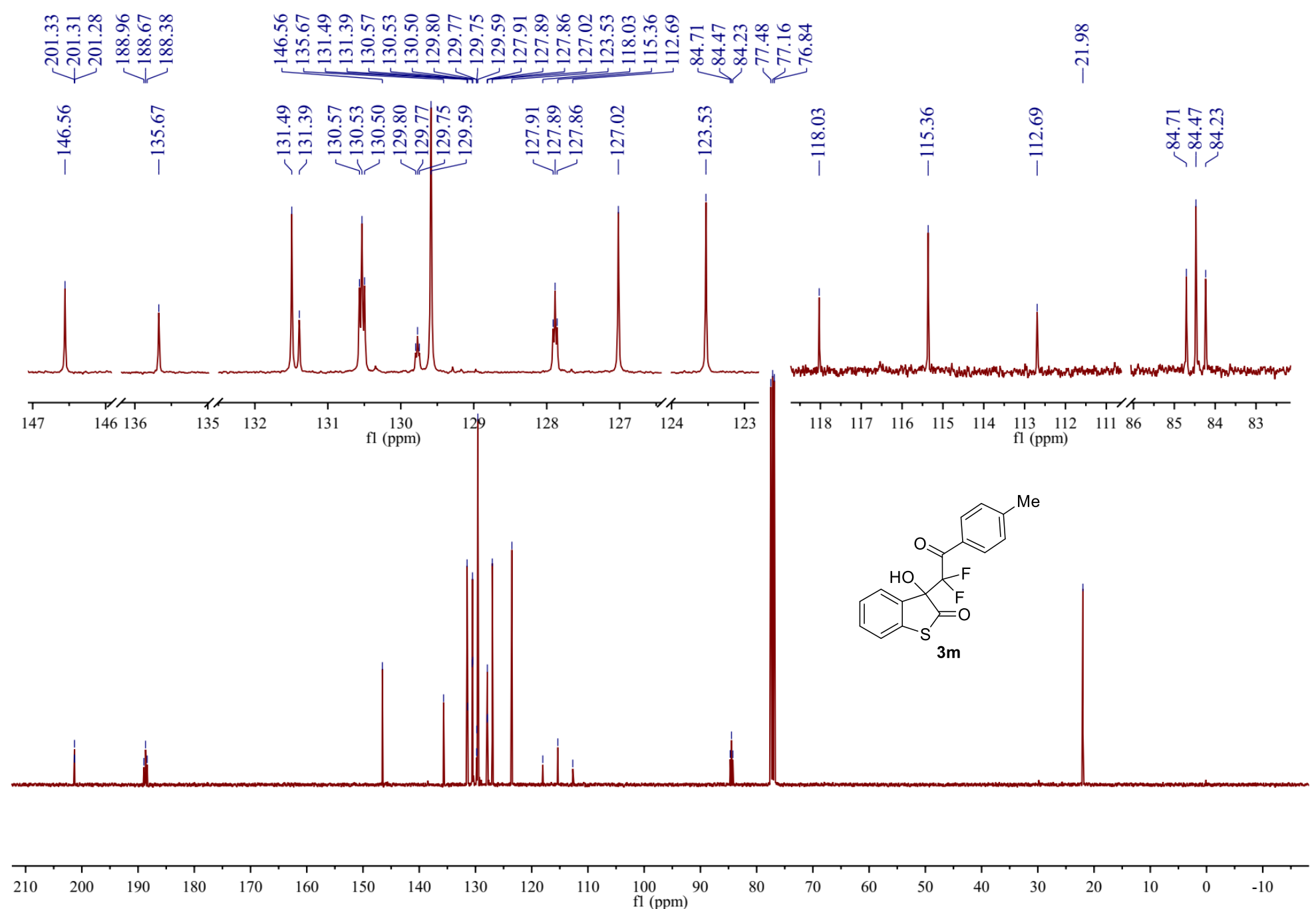

${ }^{13} \mathrm{C}\left\{{ }^{1} \mathrm{H}\right\}$ NMR spectra of $\mathbf{3 m}$ in $\mathrm{CDCl}_{3}(100 \mathrm{MHz})$ 


\section{난. \\ 宅}

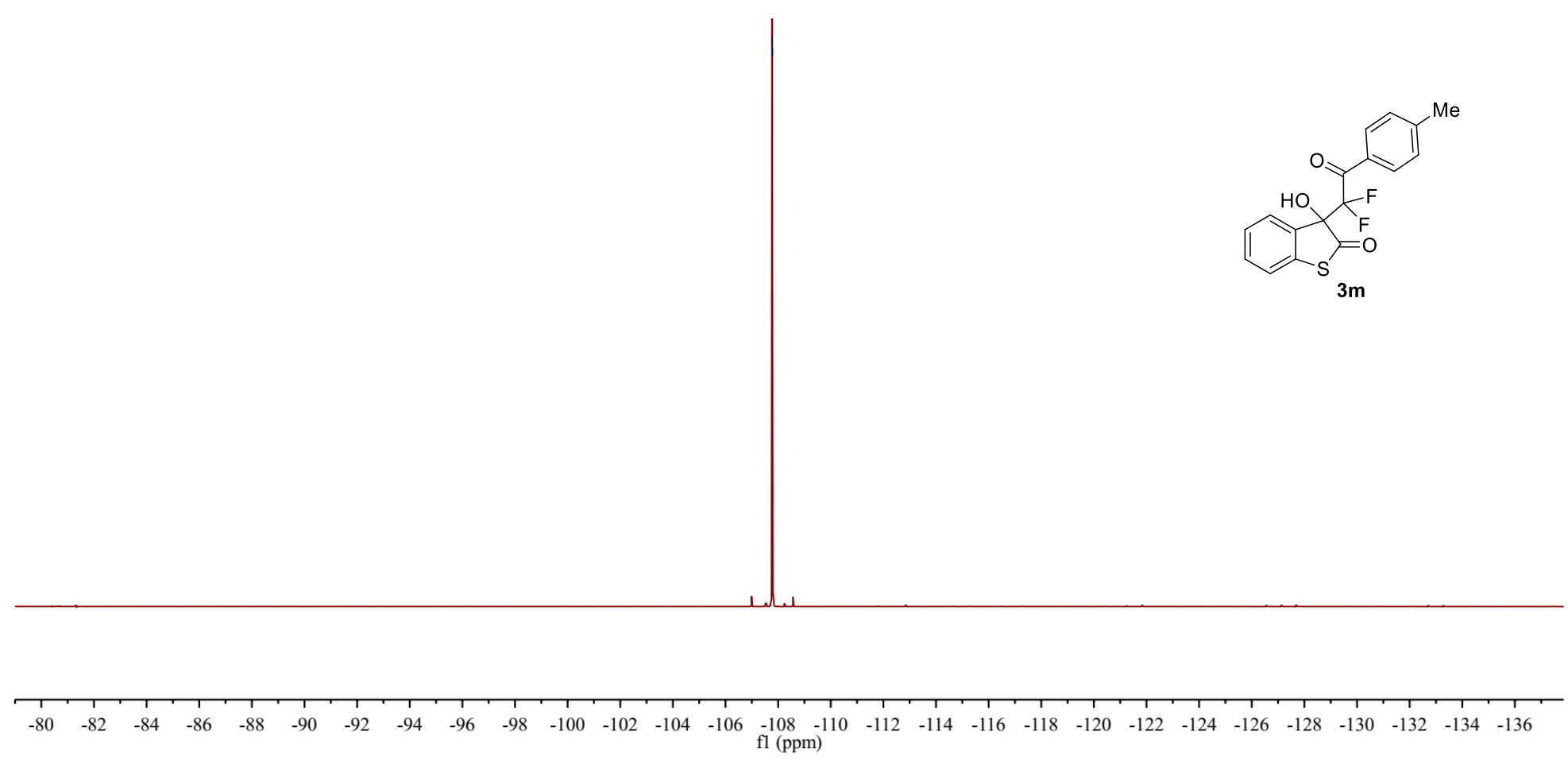

${ }^{19} \mathrm{~F}$ NMR spectra of $\mathbf{3 m}$ in $\mathrm{CDCl}_{3}(376 \mathrm{MHz})$ 

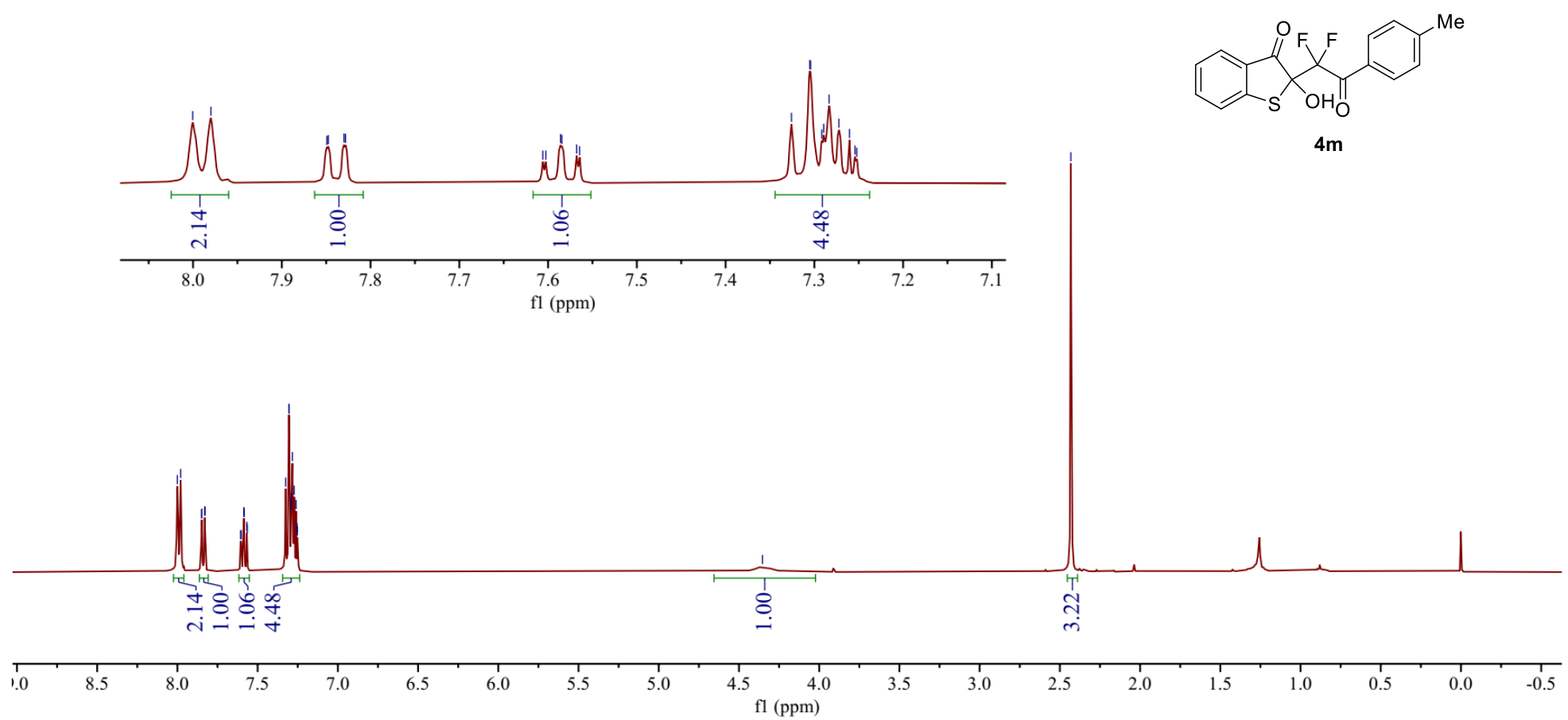

${ }^{1} \mathrm{H}$ NMR spectra of $\mathbf{4 m}$ in $\mathrm{CDCl}_{3}(400 \mathrm{MHz})$ 

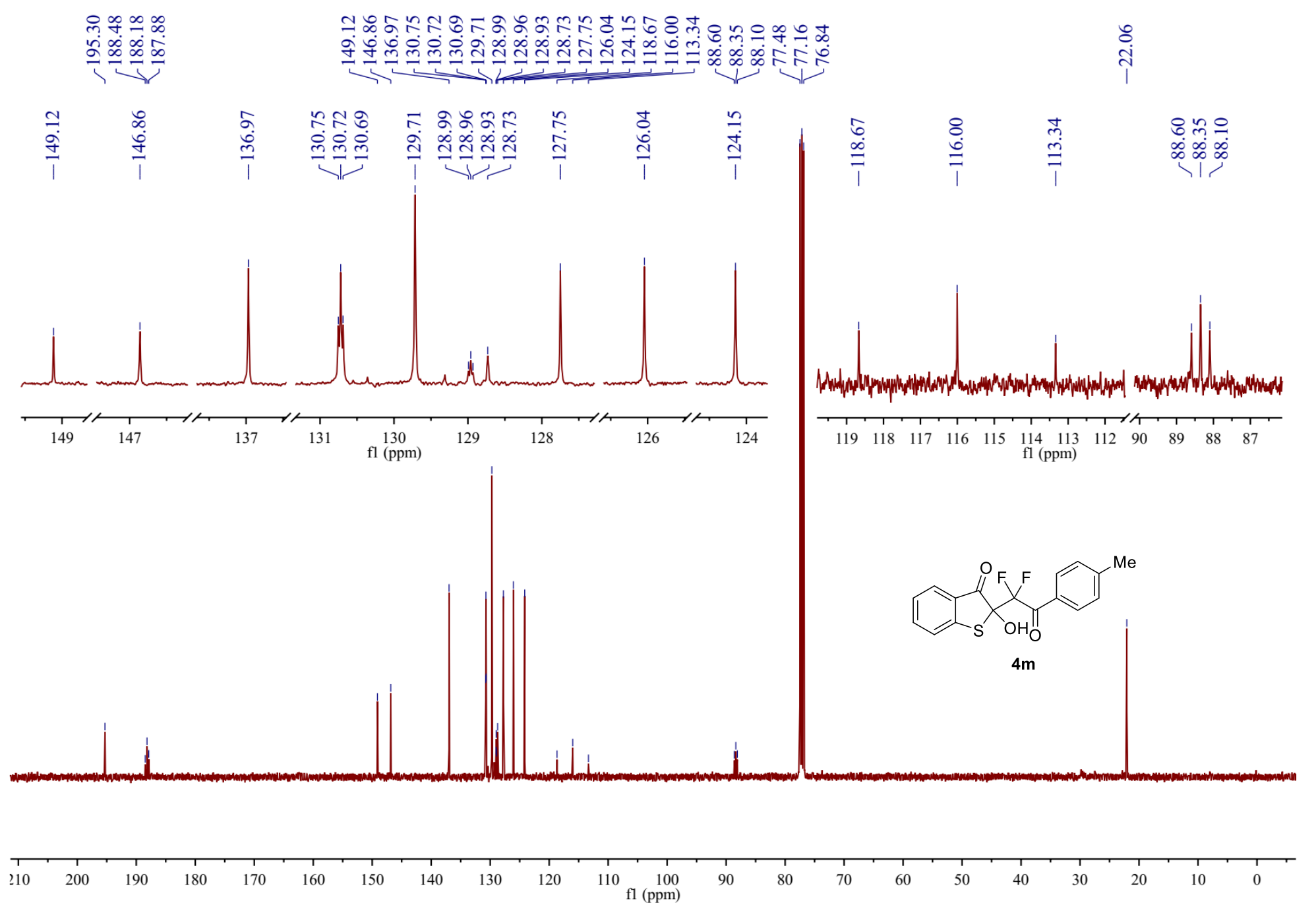

${ }^{13} \mathrm{C}\left\{{ }^{1} \mathrm{H}\right\}$ NMR spectra of $\mathbf{4 m}$ in $\mathrm{CDCl}_{3}(100 \mathrm{MHz})$ 


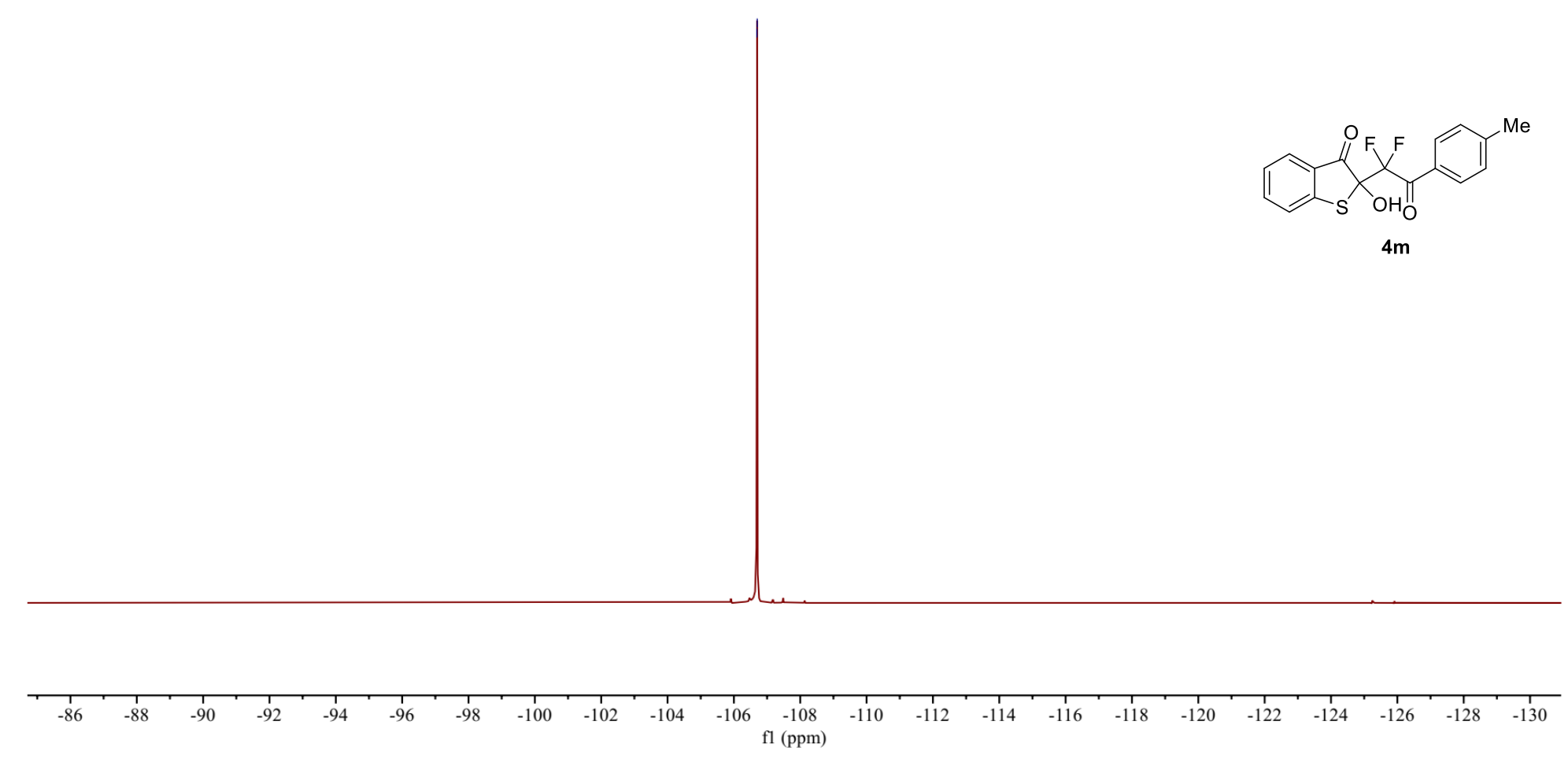

${ }^{19} \mathrm{~F}$ NMR spectra of $\mathbf{4 m}$ in $\mathrm{CDCl}_{3}(376 \mathrm{MHz})$ 
ำ

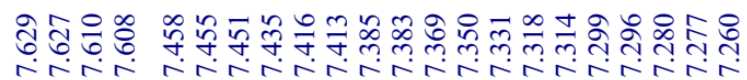

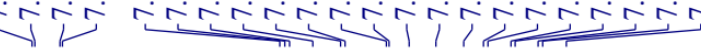

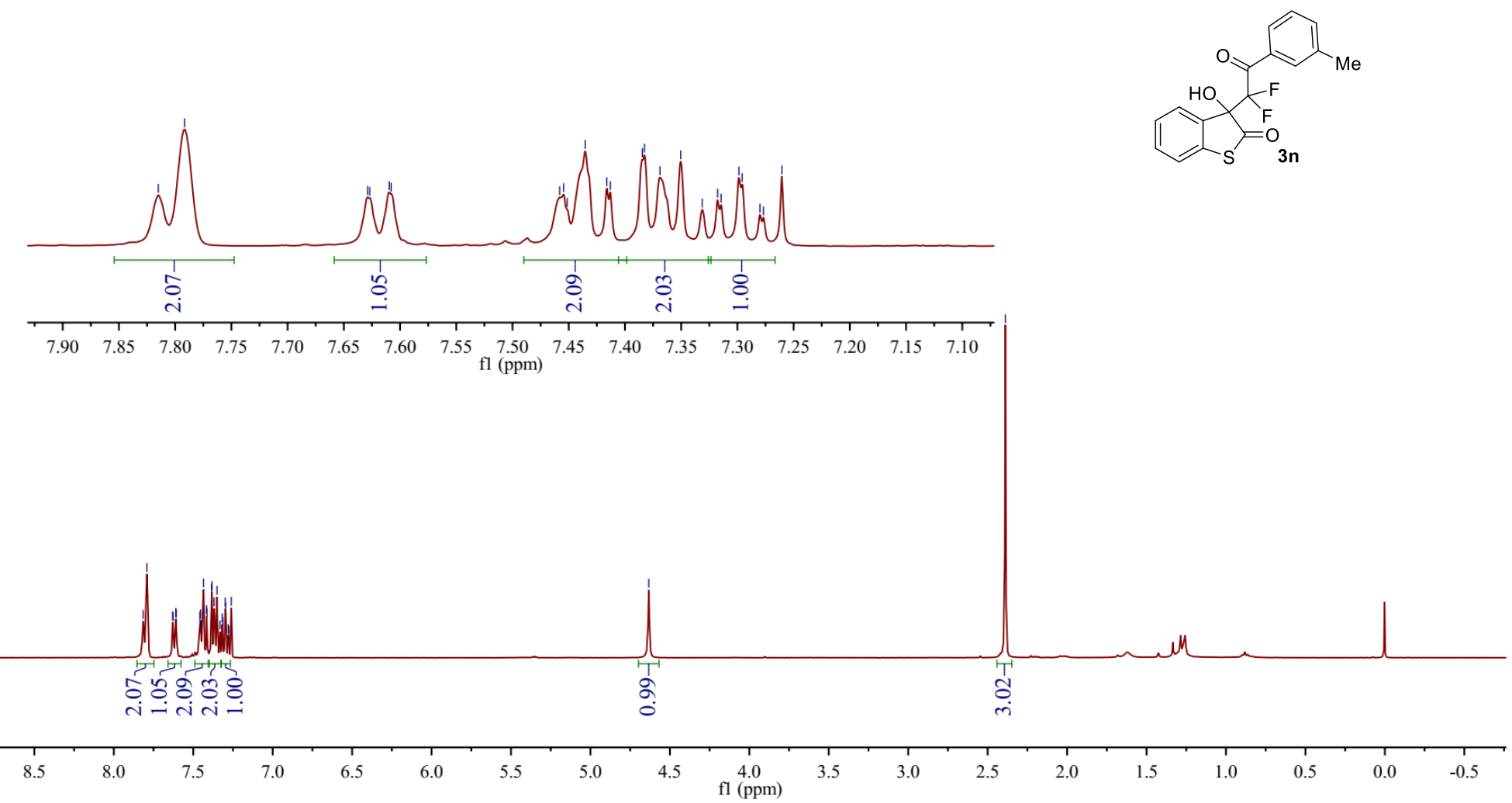

${ }^{1} \mathrm{H}$ NMR spectra of $\mathbf{3 n}$ in $\mathrm{CDCl}_{3}(400 \mathrm{MHz})$ 


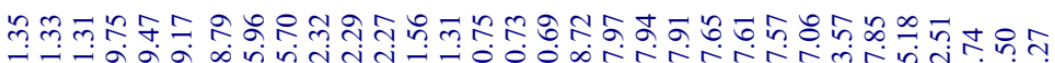

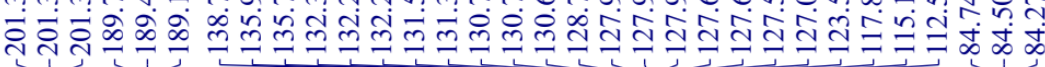

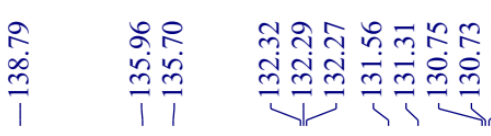

초ำ

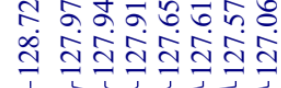
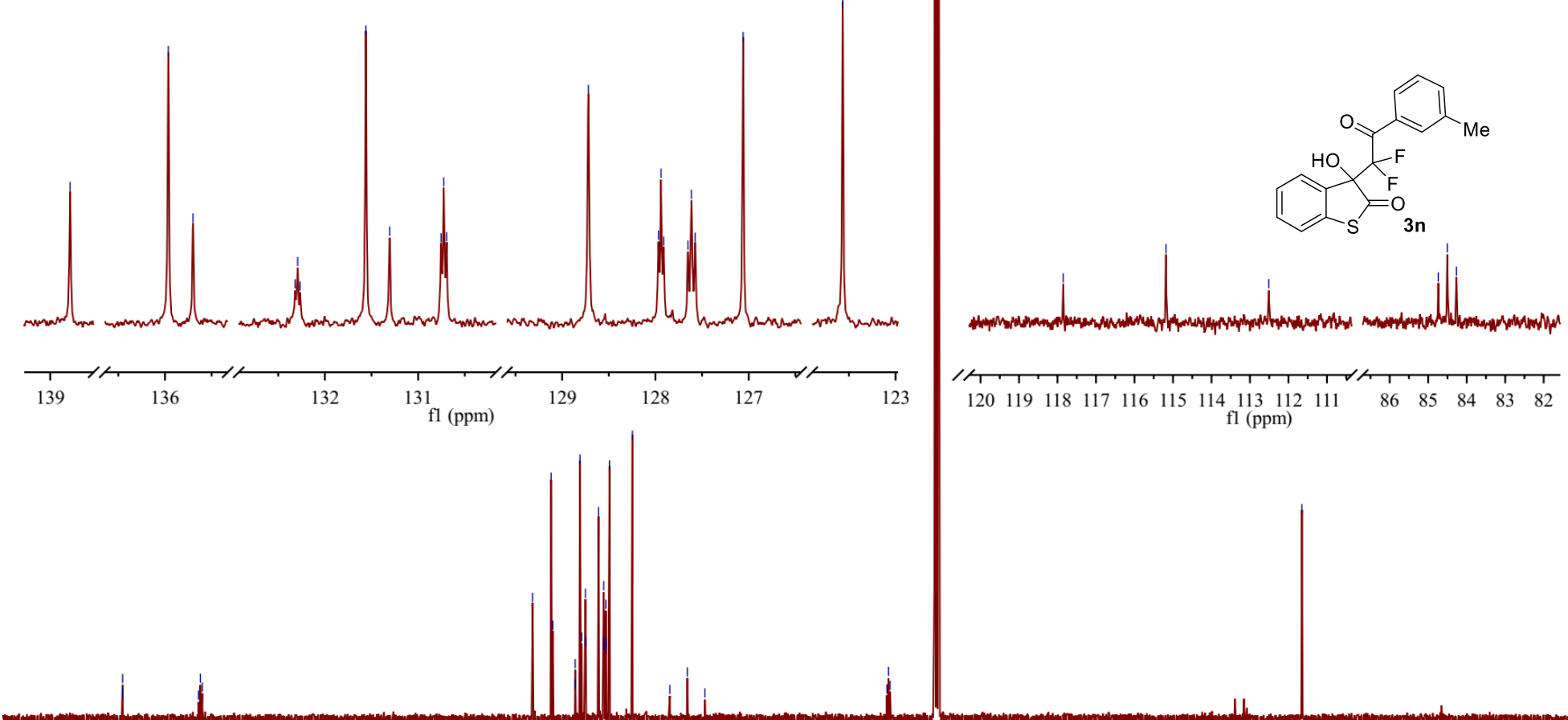

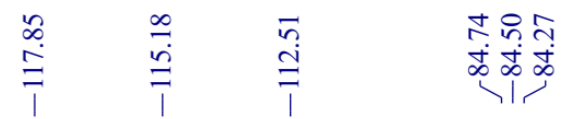

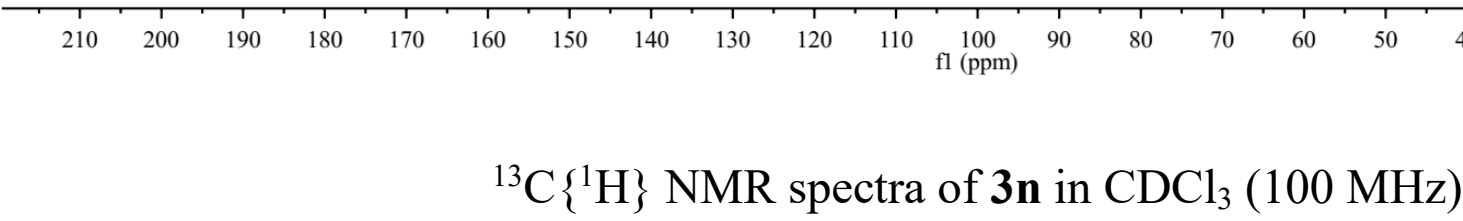



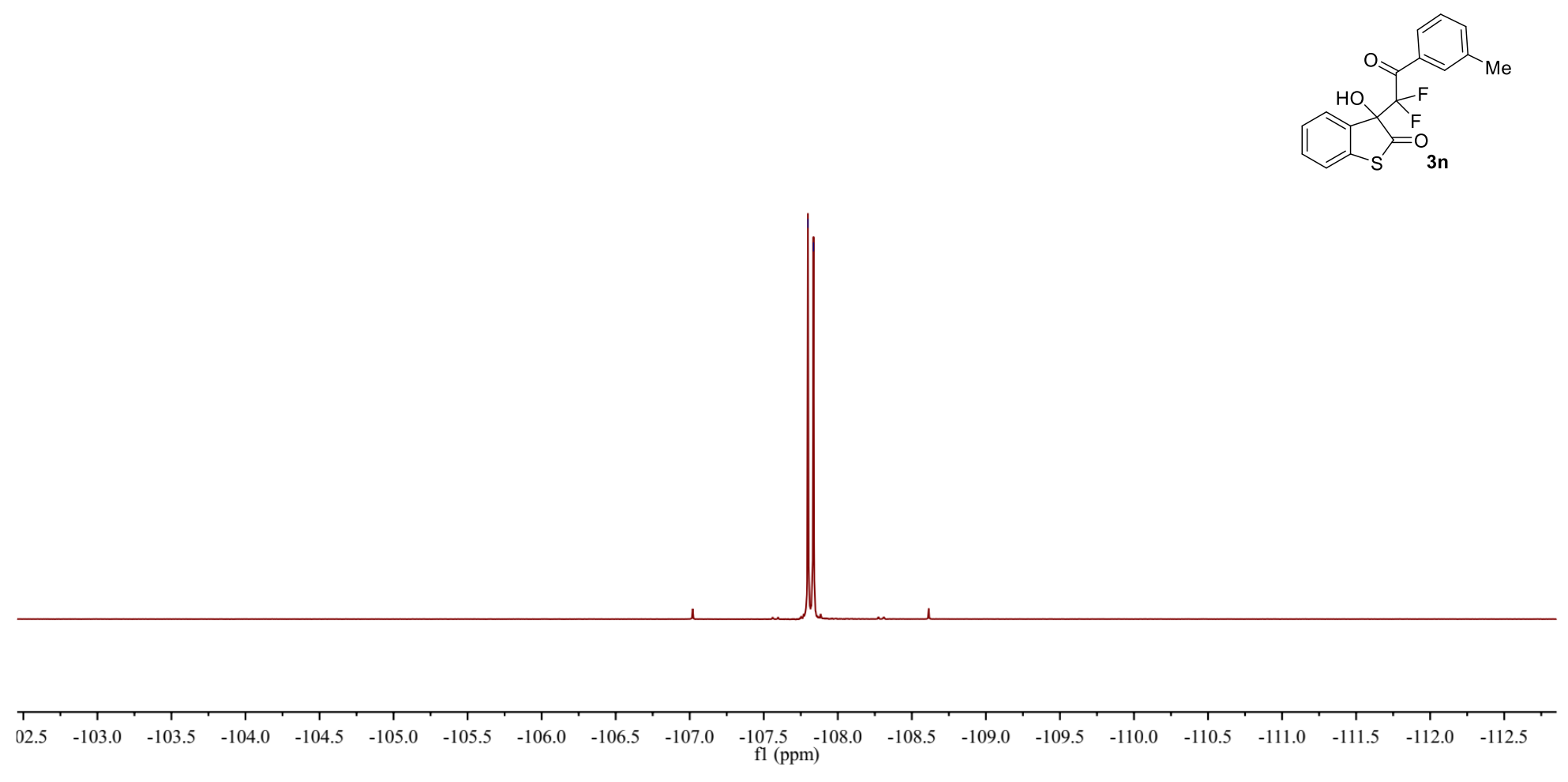

${ }^{19} \mathrm{~F}$ NMR spectra of $\mathbf{3 n}$ in $\mathrm{CDCl}_{3}(376 \mathrm{MHz})$ 


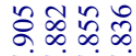

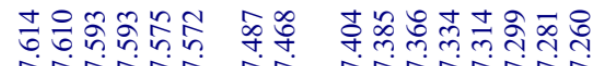

Ti个

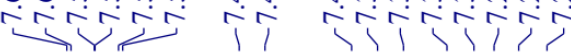
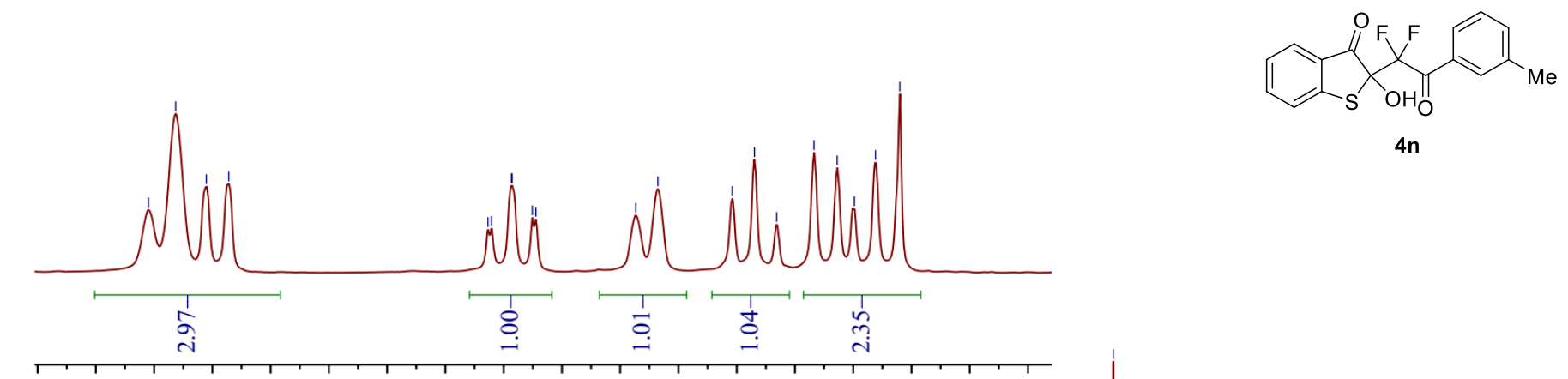
$\begin{array}{lllllllllllllllllllllll}8.00 & 7.95 & 7.90 & 7.85 & 7.80 & 7.75 & 7.70 & 7.65 & 7.60 & 7.55 & 7.50 & 7.45 & 7.40 & 7.35 & 7.30 & 7.25 & 7.20 & 7.15\end{array}$

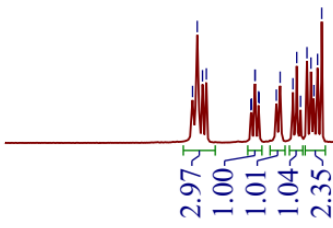

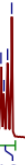

7.5

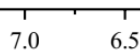

6.0

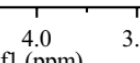

${ }^{1} \mathrm{H}$ NMR spectra of $\mathbf{4 n}$ in $\mathrm{CDCl}_{3}(400 \mathrm{MHz})$ 

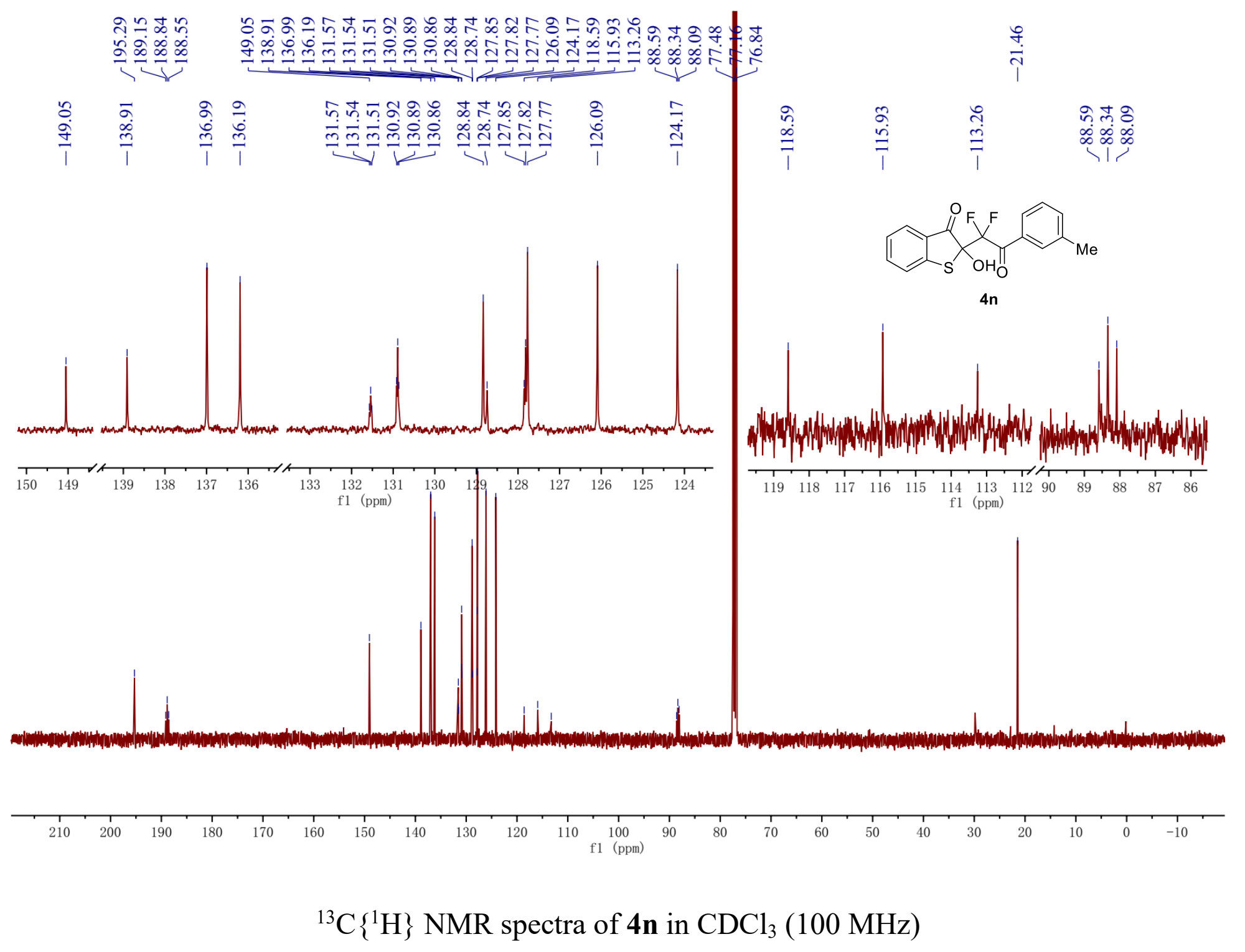


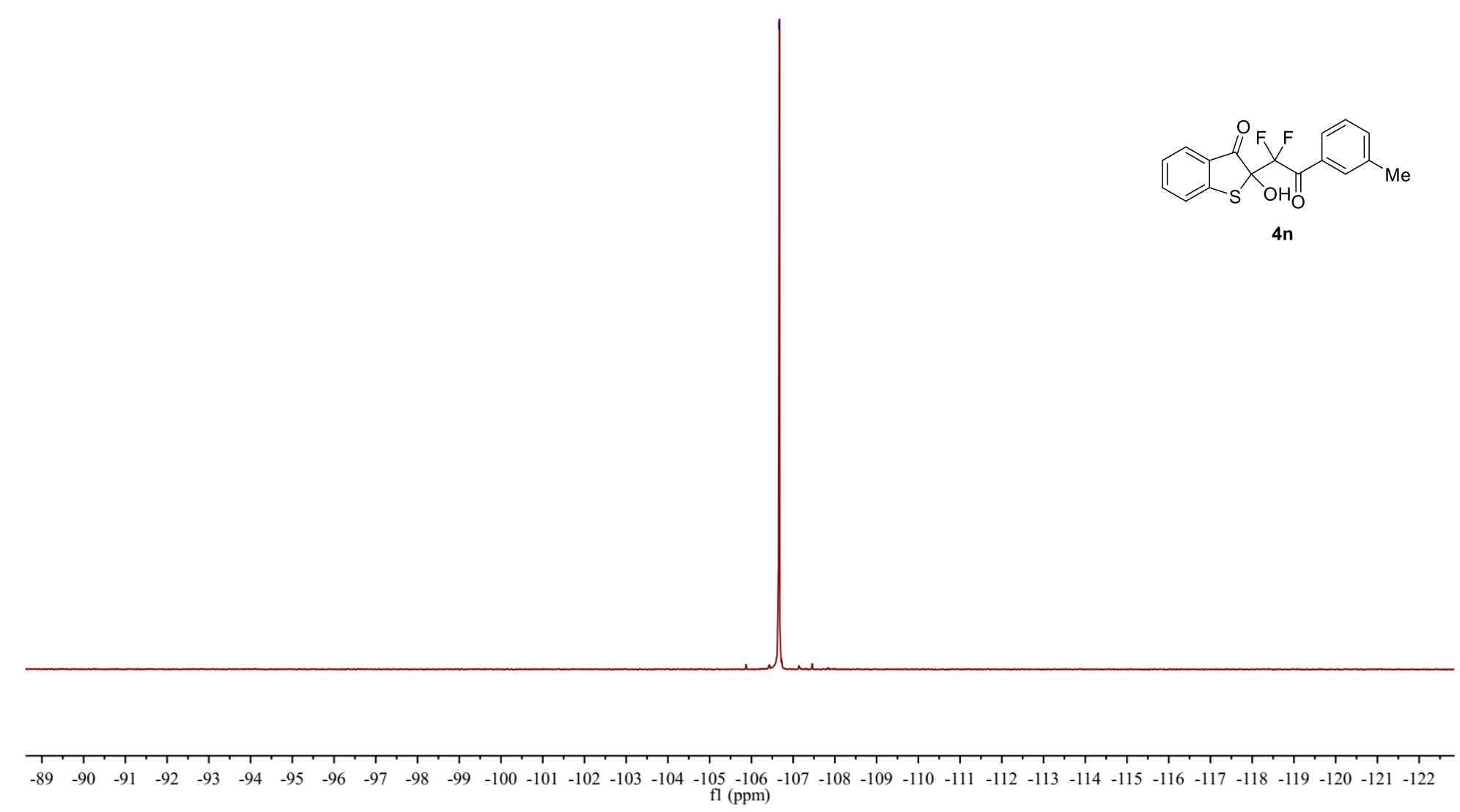

${ }^{19} \mathrm{~F}$ NMR spectra of $\mathbf{4 n}$ in $\mathrm{CDCl}_{3}(376 \mathrm{MHz})$ 


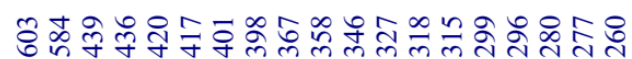

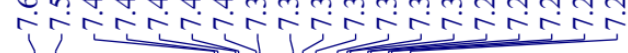

กูสูส

१广

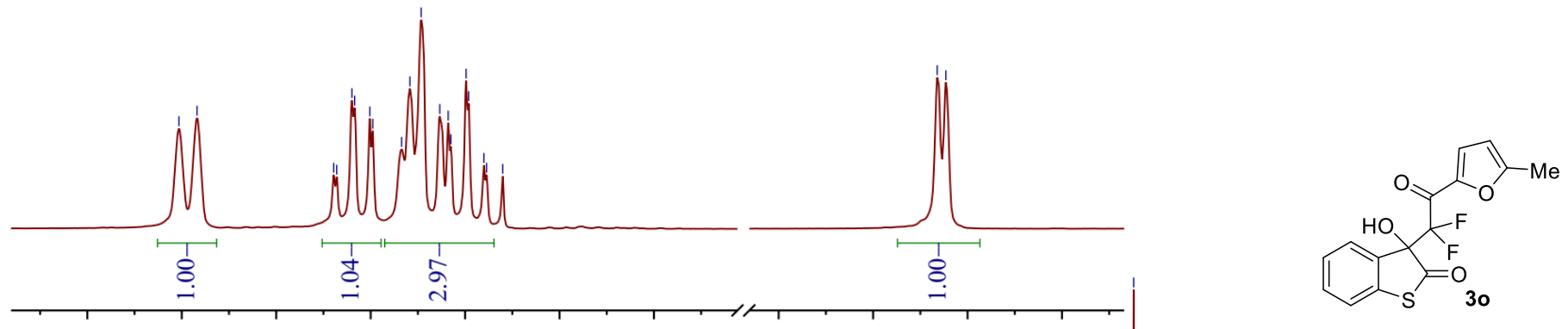

$\begin{array}{llllll}7.7 & 7.6 & 7.5 & 7.4 & 7.3 & \begin{array}{c}7.2 \\ \mathrm{fl}(\mathrm{ppm})\end{array} \\ & & & & & \end{array}$

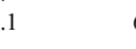

$6.4 \quad 6.3$

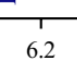

6.1

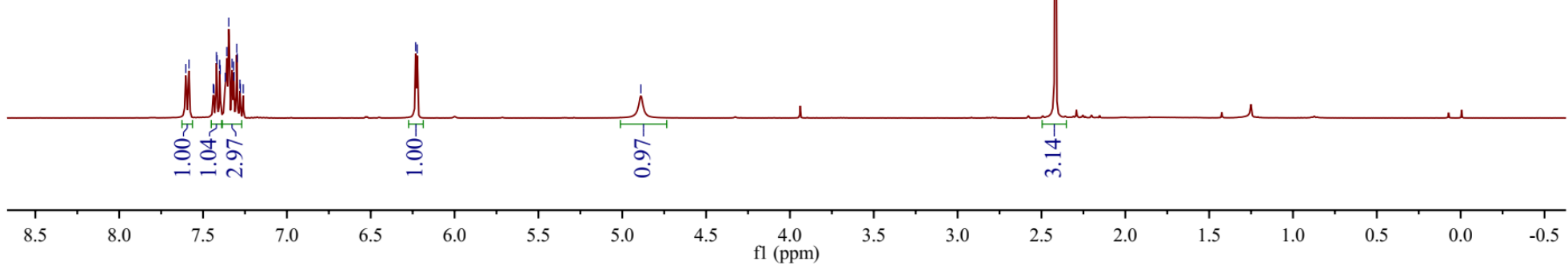

${ }^{1} \mathrm{H}$ NMR spectra of 30 in $\mathrm{CDCl}_{3}(400 \mathrm{MHz})$ 

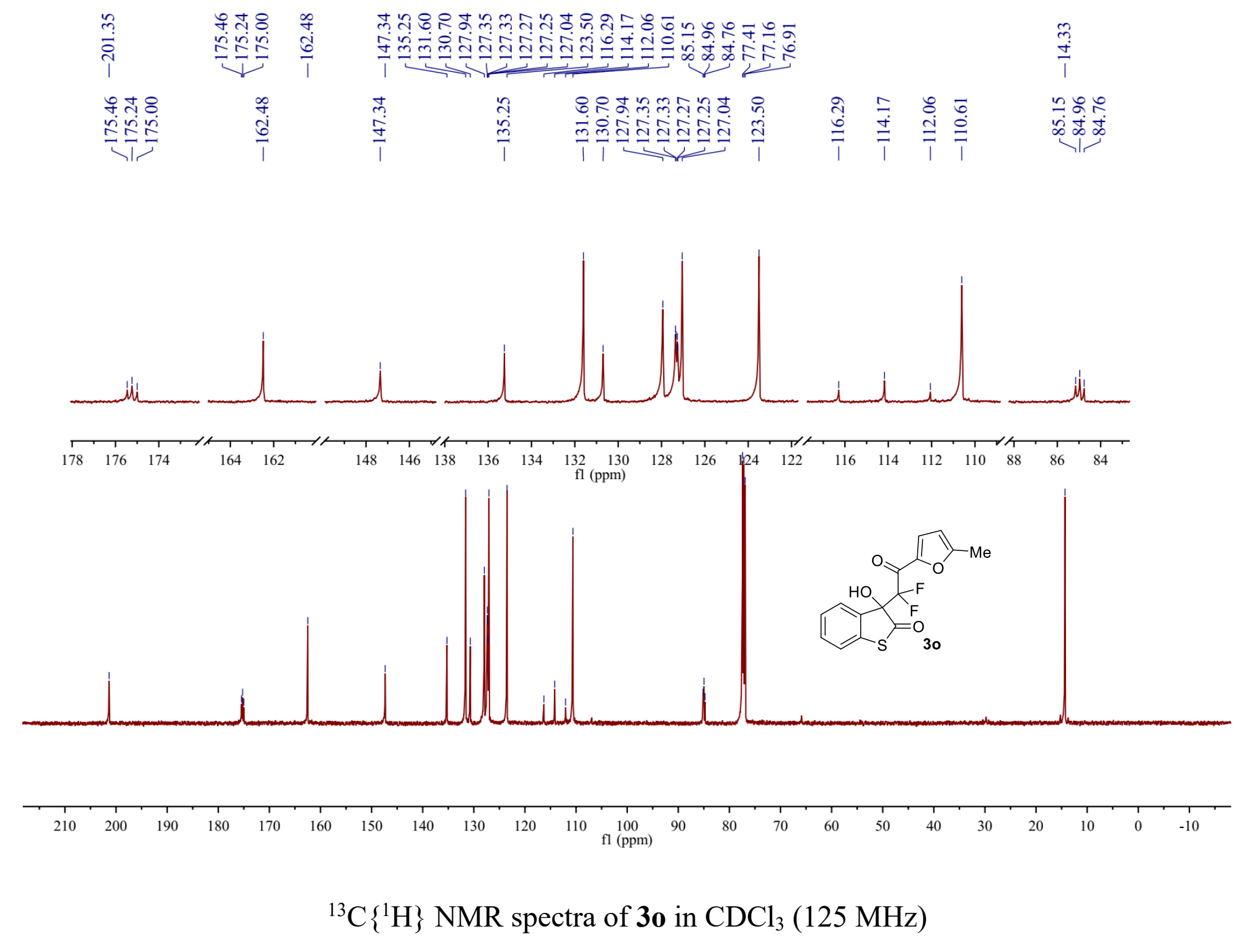


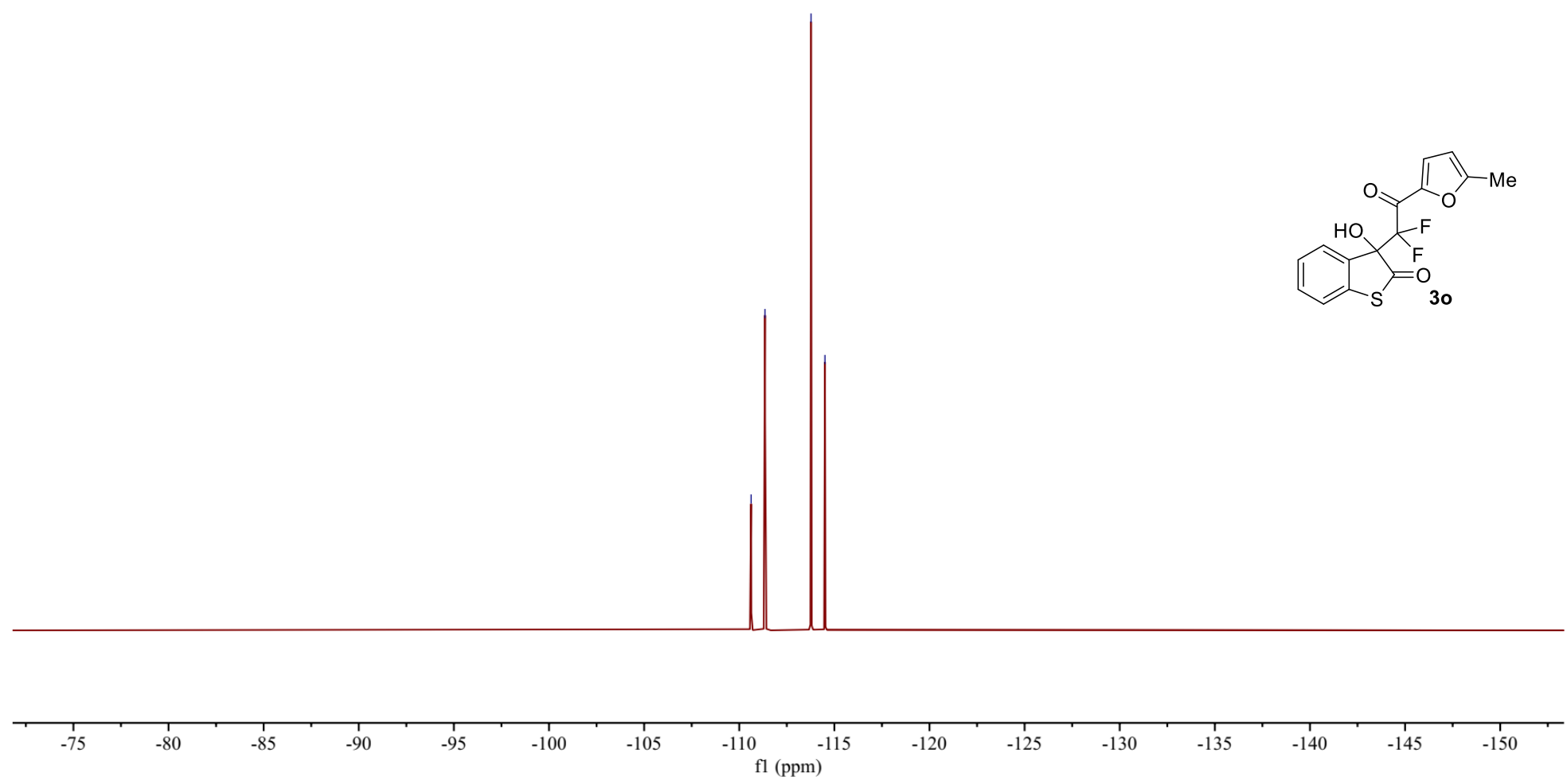

${ }^{19} \mathrm{~F}$ NMR spectra of $\mathbf{3 o}$ in $\mathrm{CDCl}_{3}(376 \mathrm{MHz})$ 


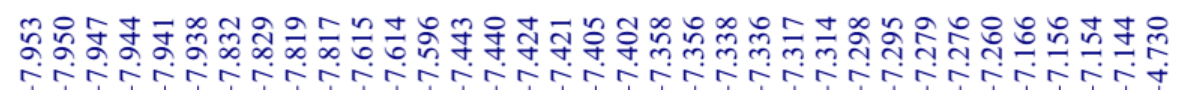

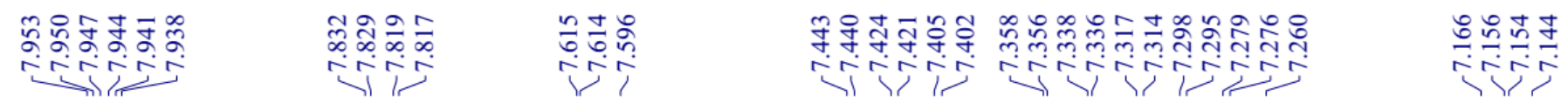
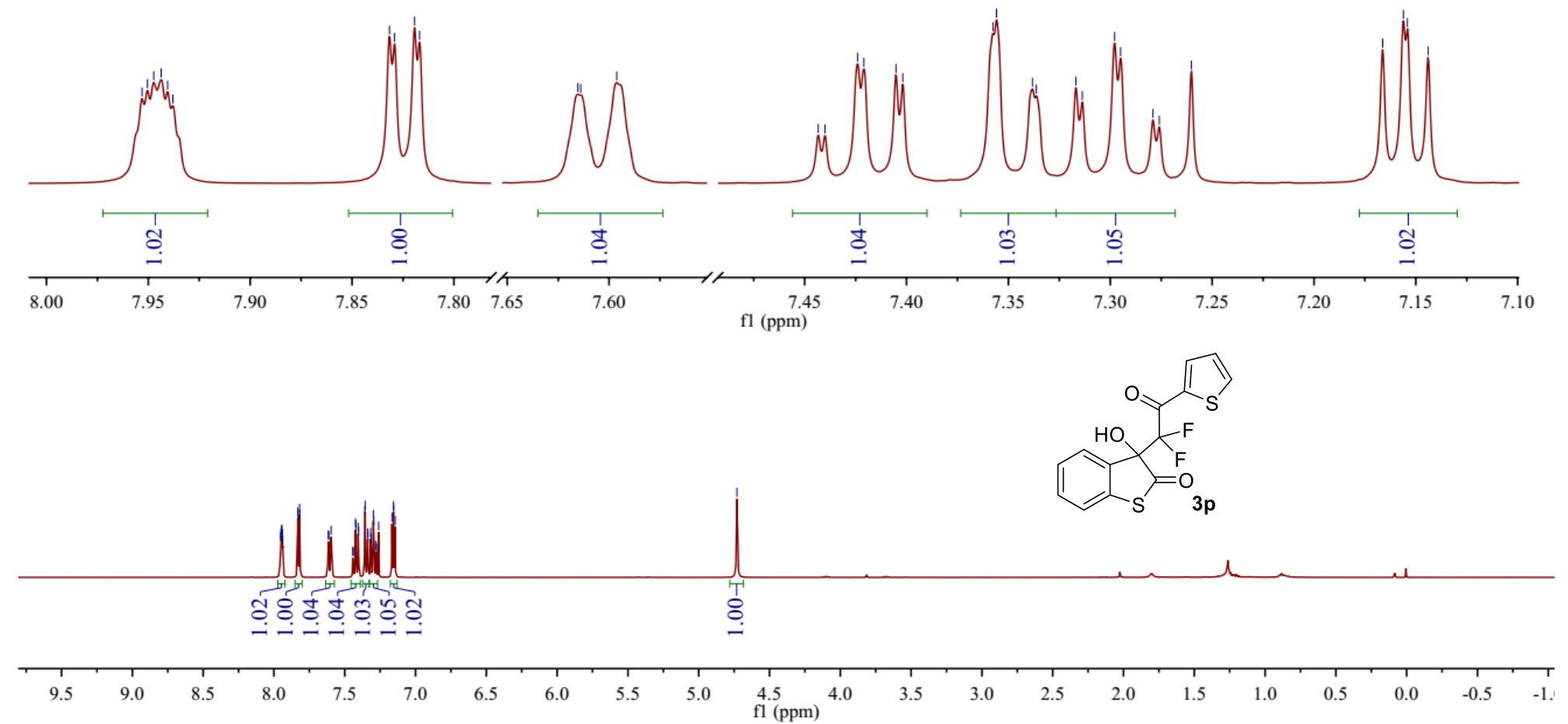

${ }^{1} \mathrm{H}$ NMR spectra of $\mathbf{3 p}$ in $\mathrm{CDCl}_{3}(400 \mathrm{MHz})$ 


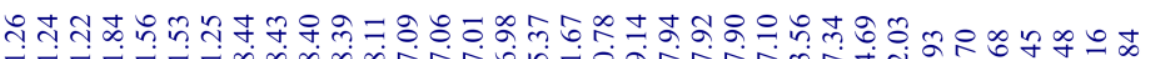

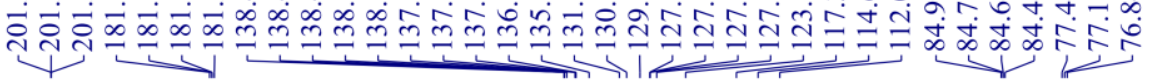
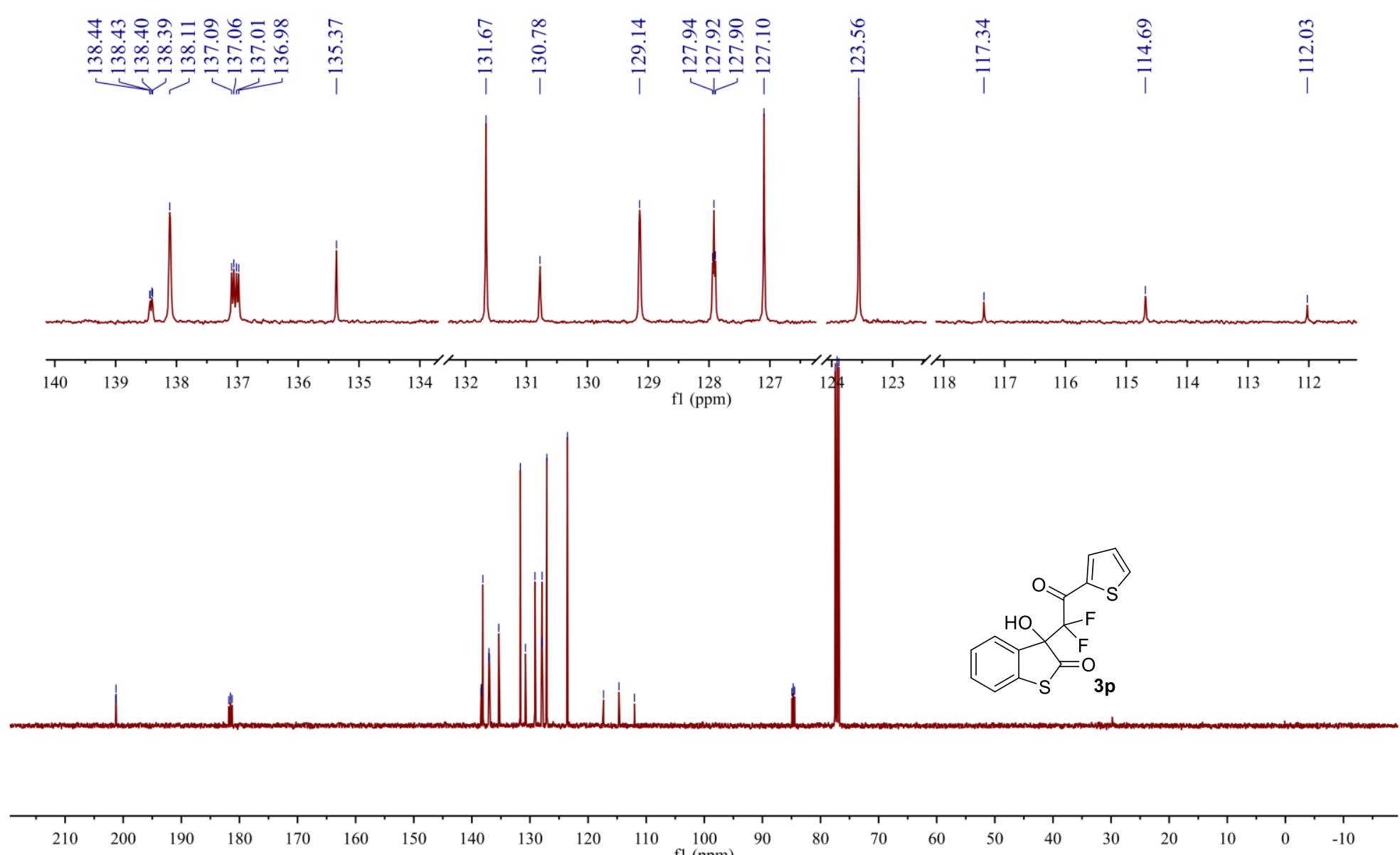

$\begin{array}{ll}60 & 150\end{array}$

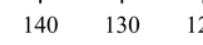

$110 \quad \begin{aligned} & 100 \\ & \mathrm{fl}(\mathrm{ppm})\end{aligned}$
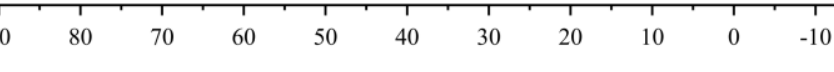

${ }^{13} \mathrm{C}\left\{{ }^{1} \mathrm{H}\right\}$ NMR spectra of $\mathbf{3 p}$ in $\mathrm{CDCl}_{3}(100 \mathrm{MHz})$ 


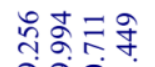

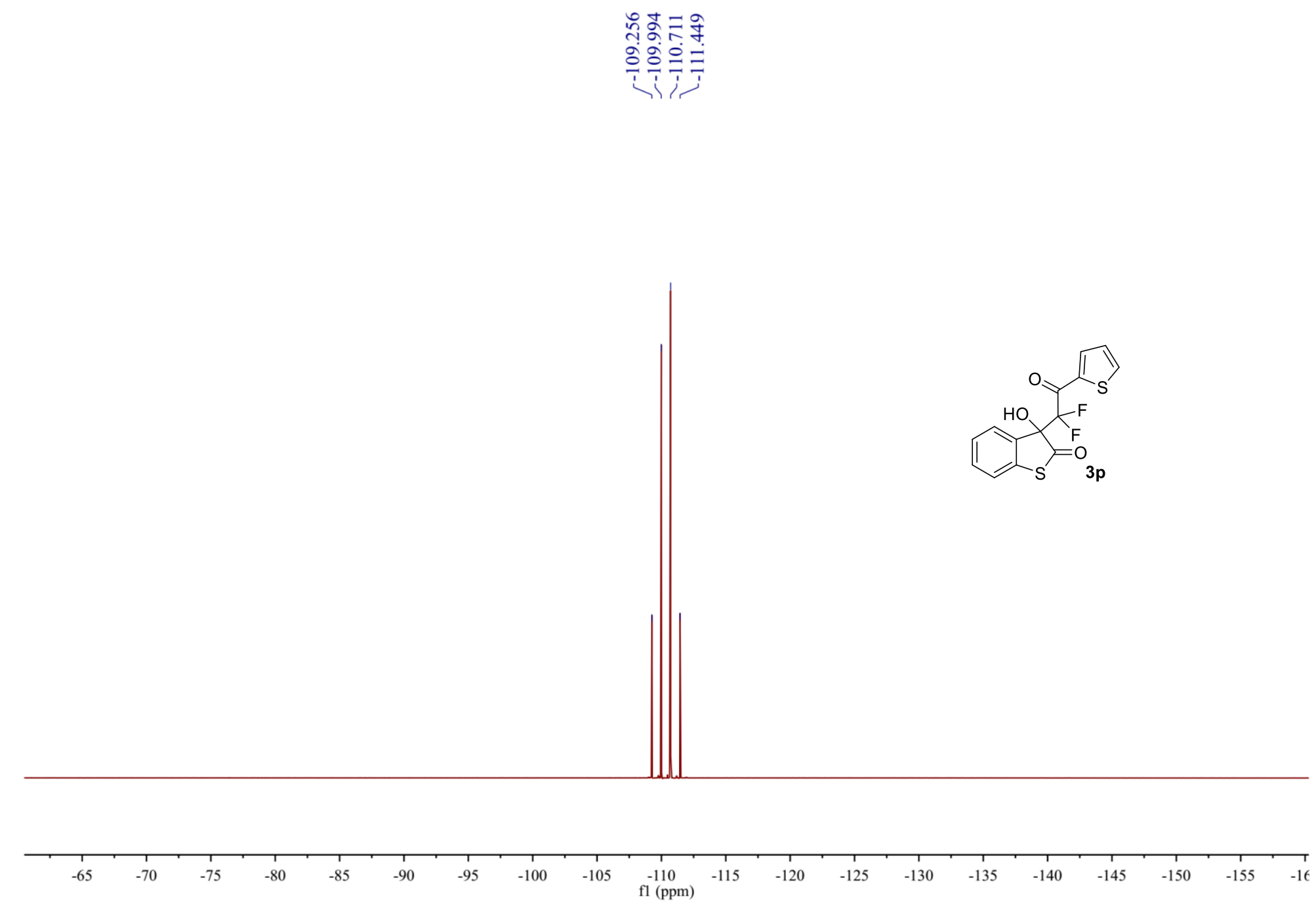

${ }^{19} \mathrm{~F}$ NMR spectra of $\mathbf{3 p}$ in $\mathrm{CDCl}_{3}(376 \mathrm{MHz})$ 


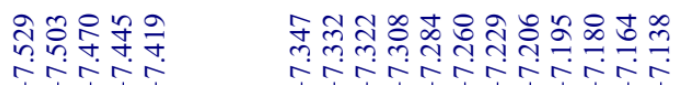

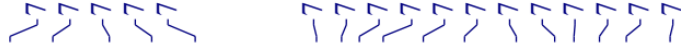
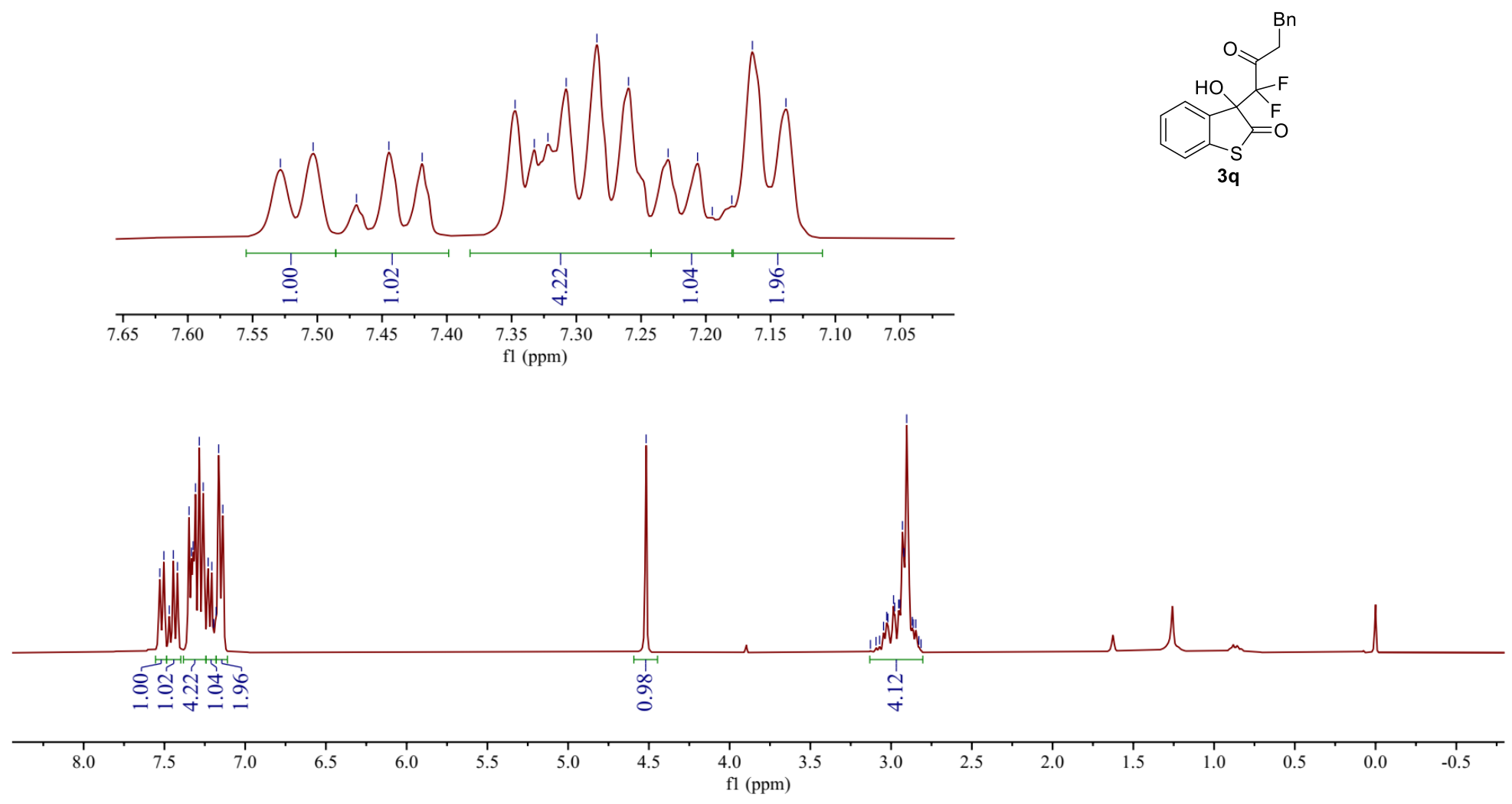

${ }^{1} \mathrm{H}$ NMR spectra of $\mathbf{3 q}$ in $\mathrm{CDCl}_{3}(400 \mathrm{MHz})$ 

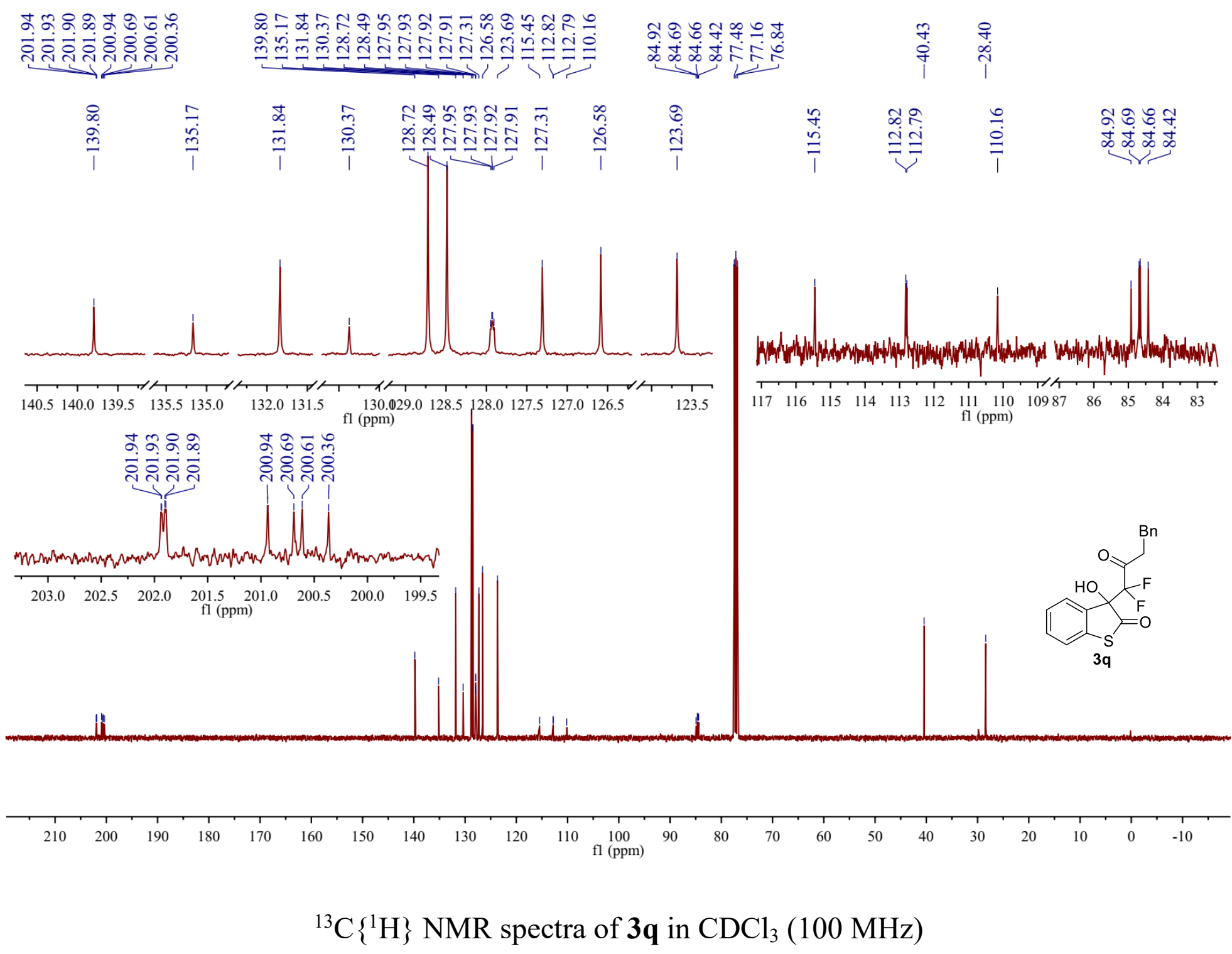


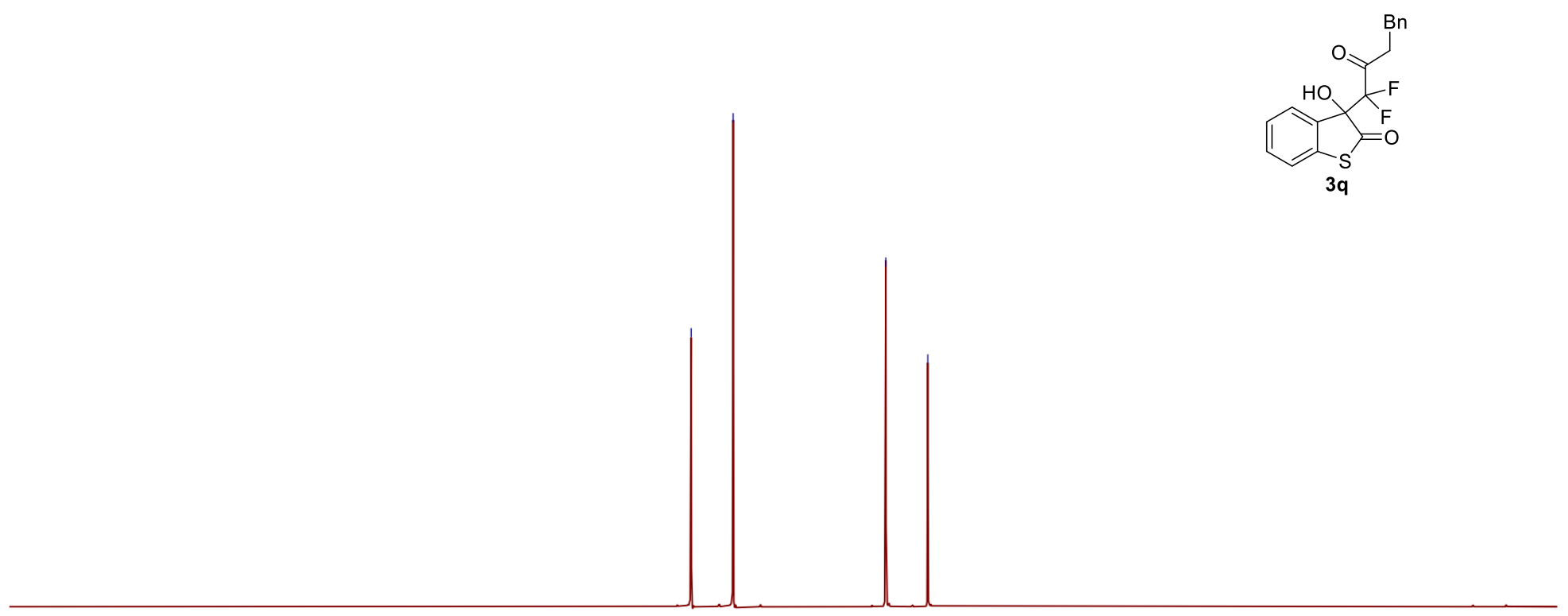

\begin{tabular}{lllllllllllllllllllllllllllllllllllllllllll}
\hline 103 & -104 & -105 & -106 & -107 & -108 & -109 & -110 & -111 & -112 & -113 & -114 & -115 & -116 & -117 & -118 & -119 & -120 & -121 & -122 & -123 & -124 & -125 & -126 & -127 & -128 & -129
\end{tabular}

${ }^{19} \mathrm{~F}$ NMR spectra of $\mathbf{3 q}$ in $\mathrm{CDCl}_{3}(376 \mathrm{MHz})$ 


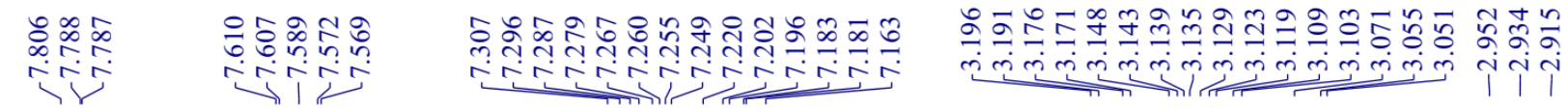
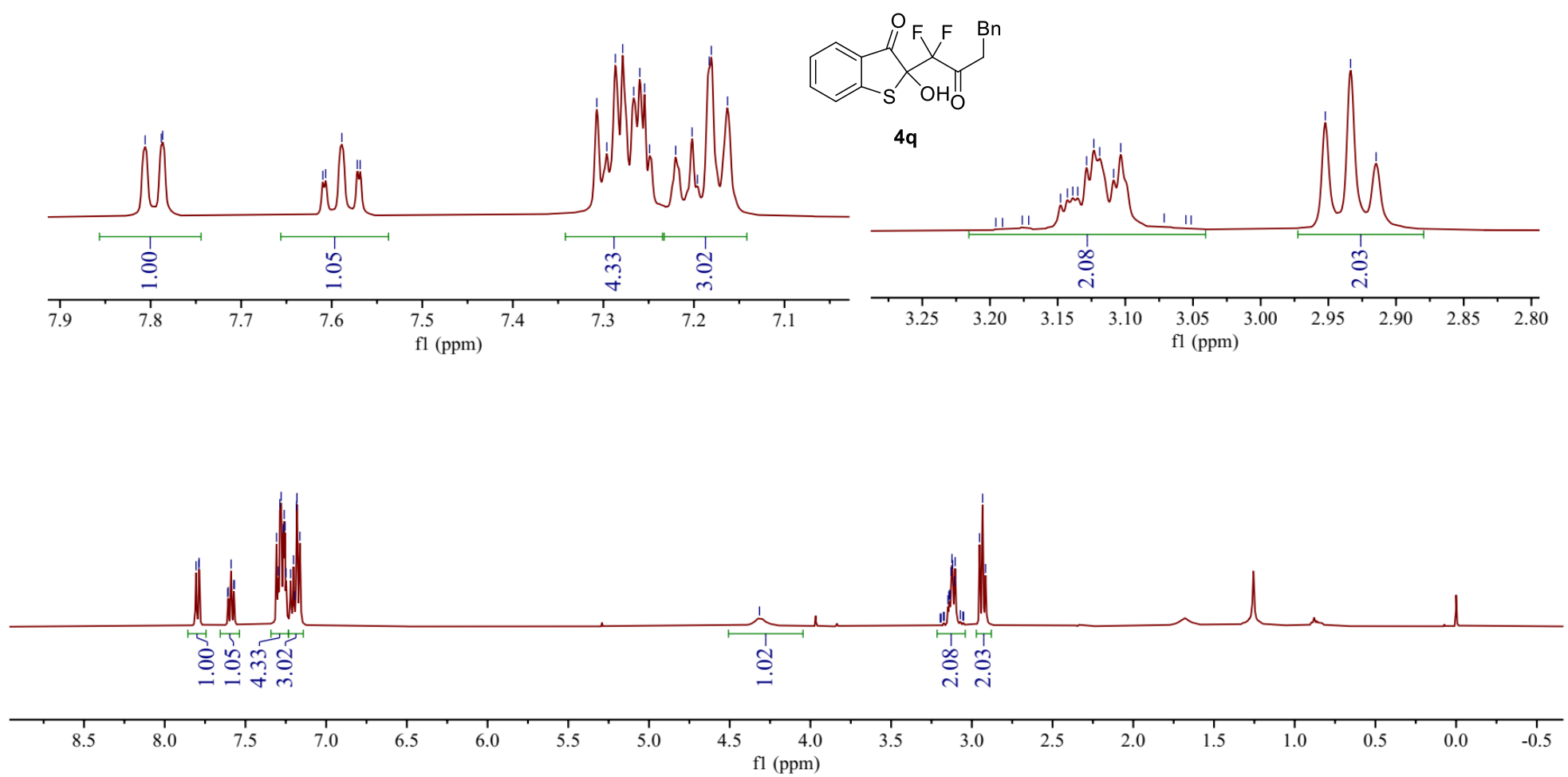

${ }^{1} \mathrm{H}$ NMR spectra of $\mathbf{4 q}$ in $\mathrm{CDCl}_{3}(400 \mathrm{MHz})$ 


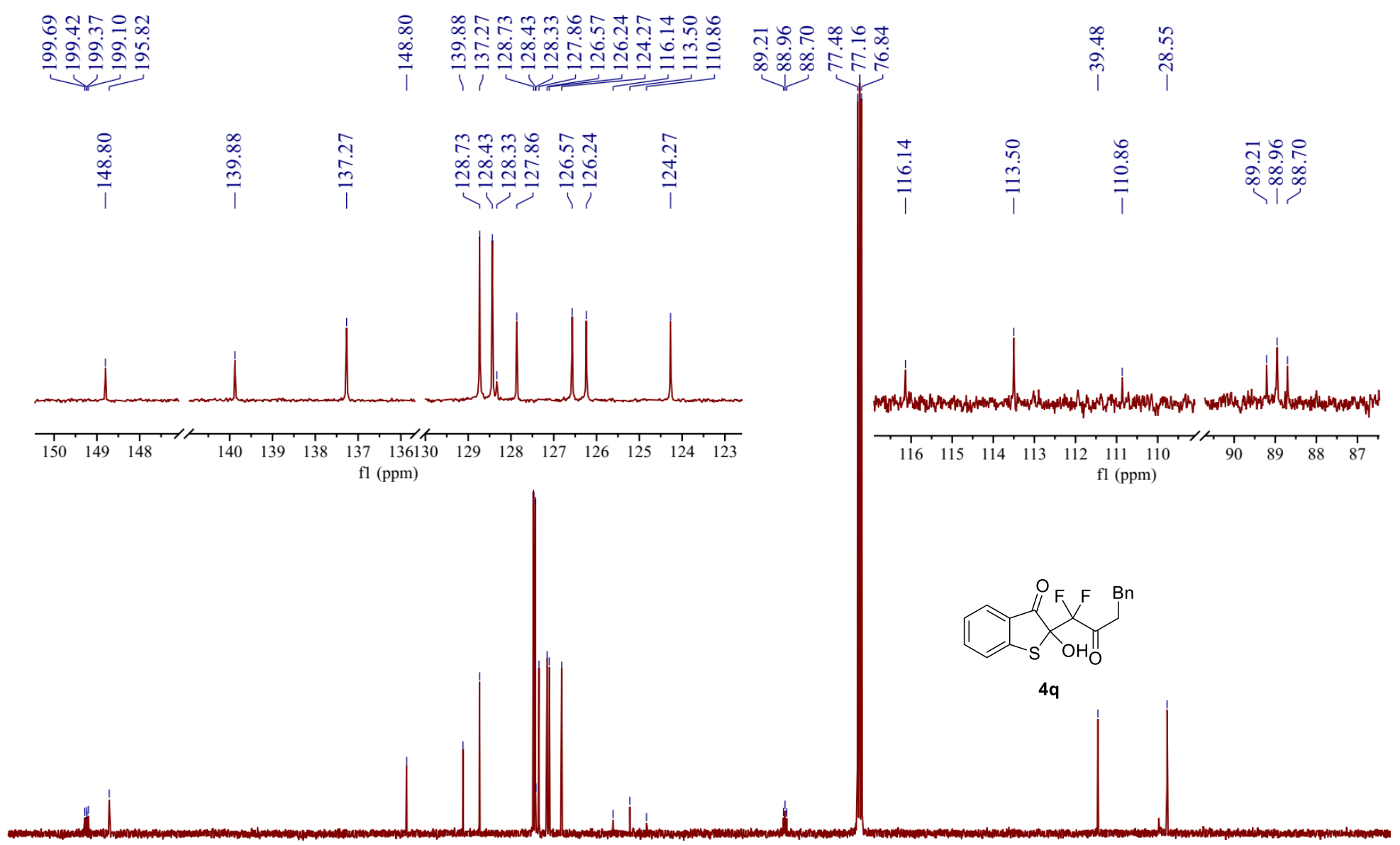

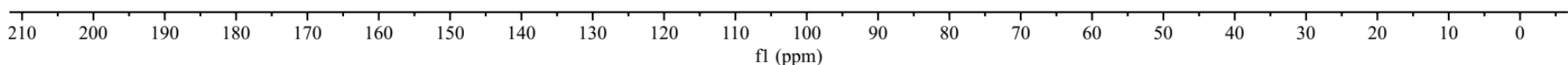

${ }^{13} \mathrm{C}\left\{{ }^{1} \mathrm{H}\right\}$ NMR spectra of $\mathbf{4 q}$ in $\mathrm{CDCl}_{3}(100 \mathrm{MHz})$ 


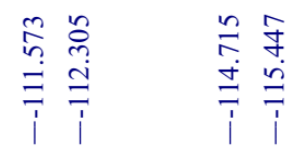

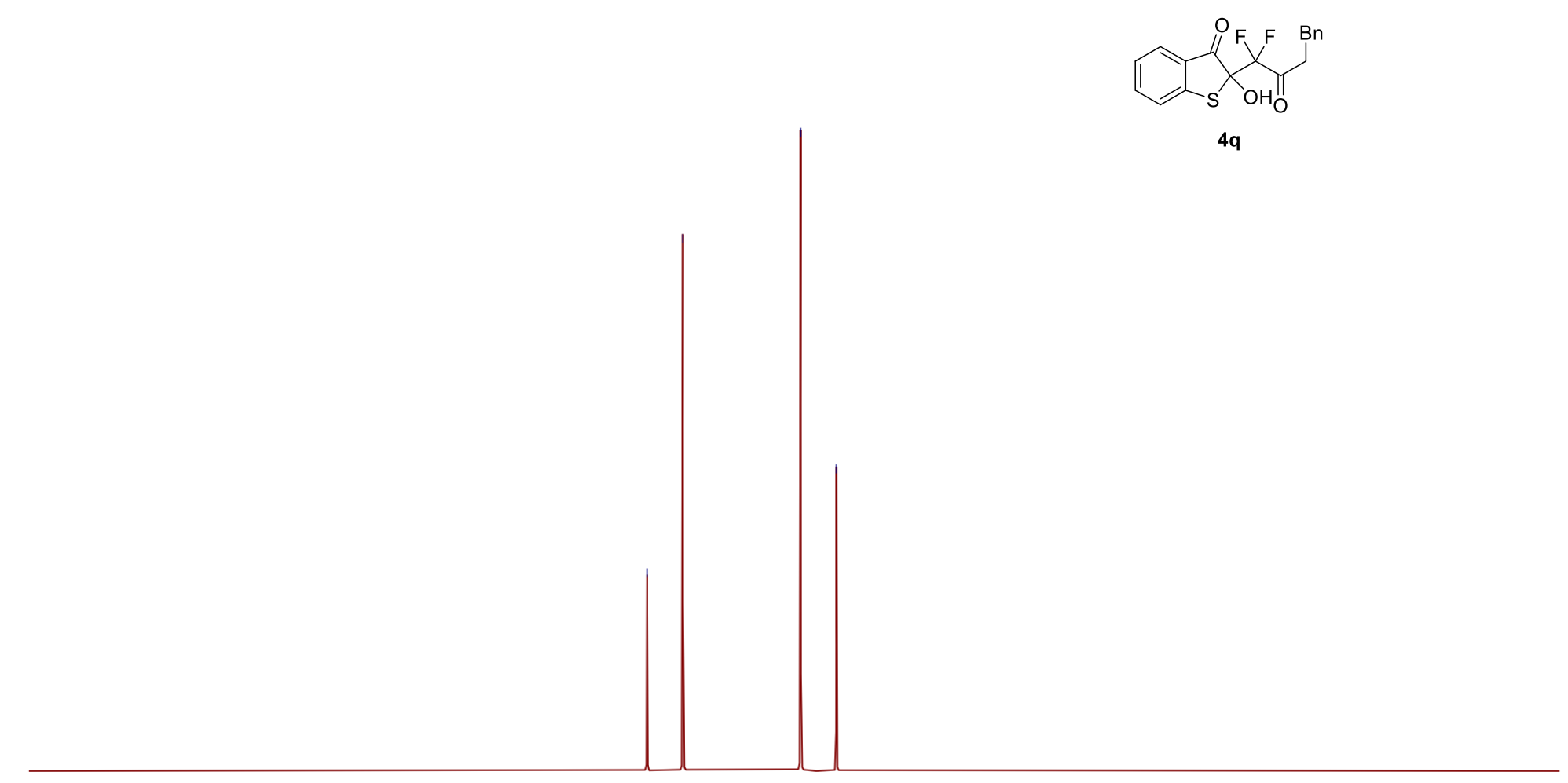

$\begin{array}{llllllllllllllllllllllllllllllllllllllllllllll}99 & -100 & -101 & -102 & -103 & -104 & -105 & -106 & -107 & -108 & -109 & -110 & -111 & -112 & -113 & -114 & -115 & -116 & -117 & -118 & -119 & -120 & -121 & -122 & -123 & -124 & -125 & -126 & -127 & -128 & -129 & -131\end{array}$

${ }^{19} \mathrm{~F}$ NMR spectra of $\mathbf{4 q}$ in $\mathrm{CDCl}_{3}(376 \mathrm{MHz})$ 


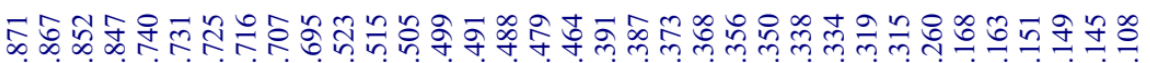

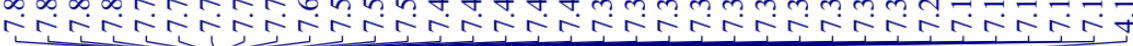

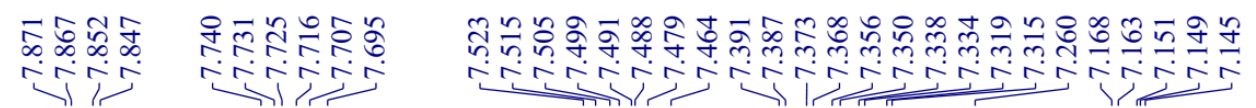

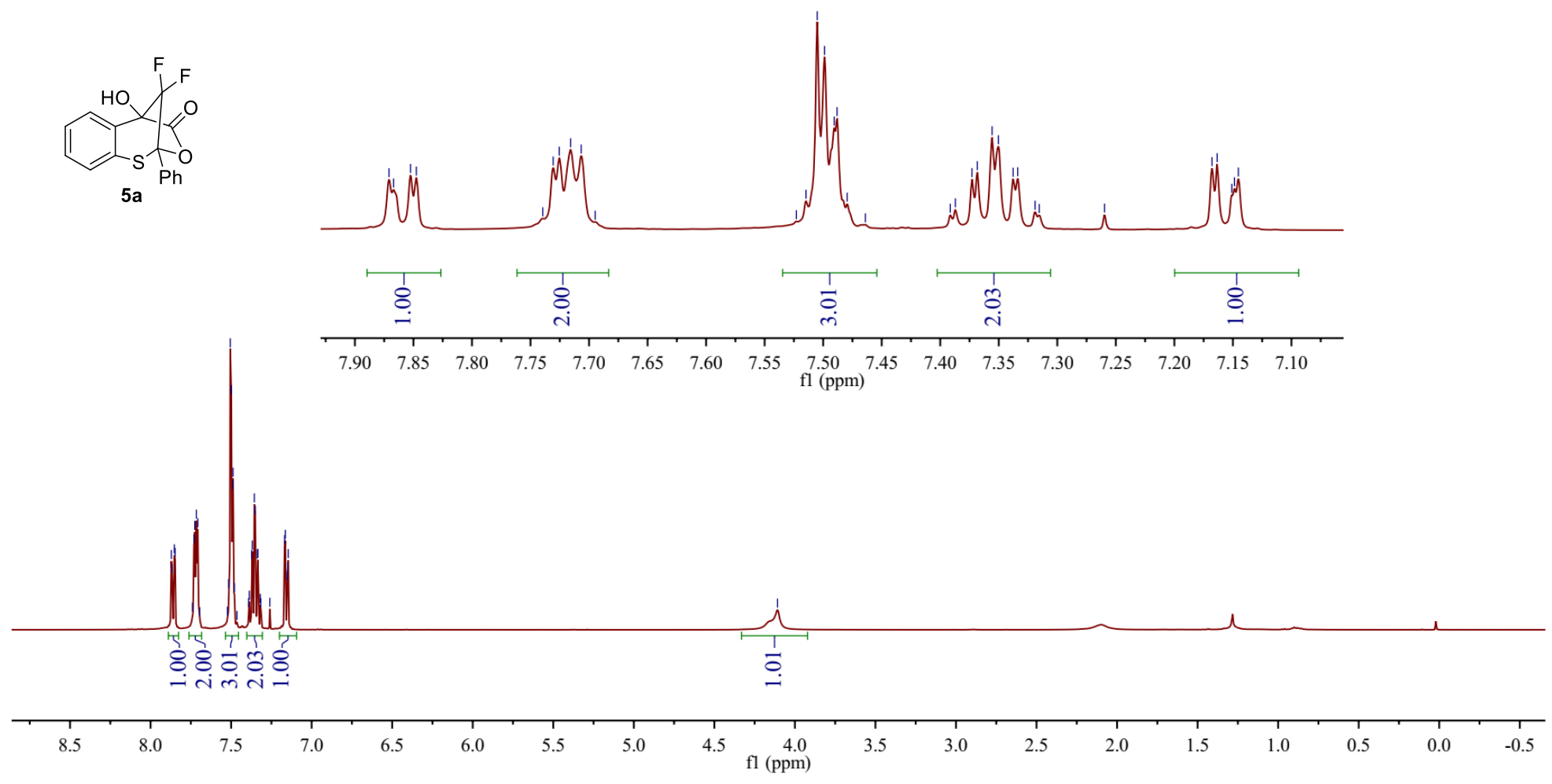

${ }^{1} \mathrm{H}$ NMR spectra of $\mathbf{5 a}$ in $\mathrm{CDCl}_{3}(400 \mathrm{MHz})$ 

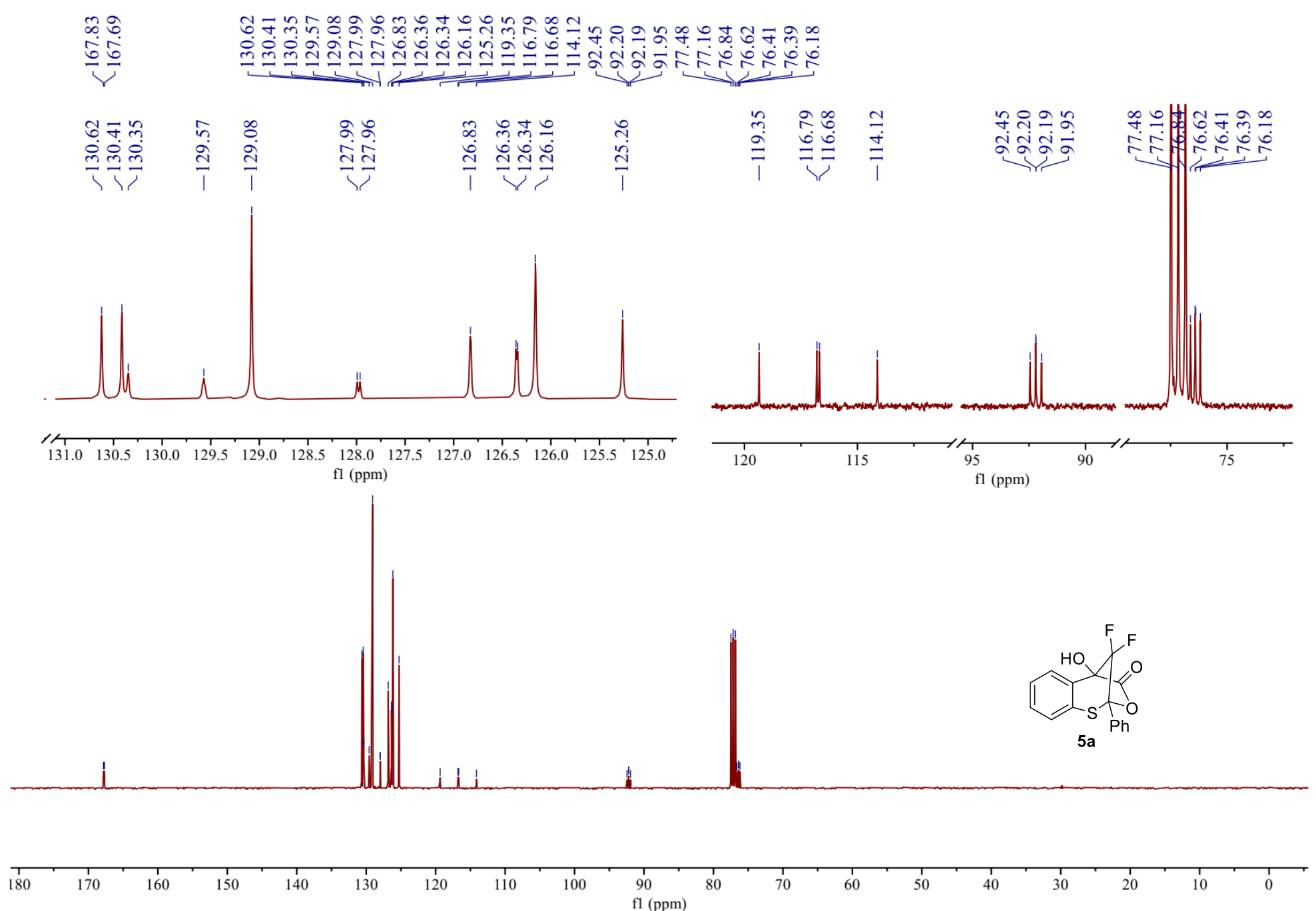

${ }^{13} \mathrm{C}\left\{{ }^{1} \mathrm{H}\right\}$ NMR spectra of $\mathbf{5 a}$ in $\mathrm{CDCl}_{3}(100 \mathrm{MHz})$ 


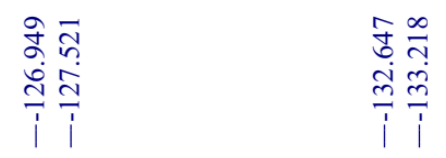
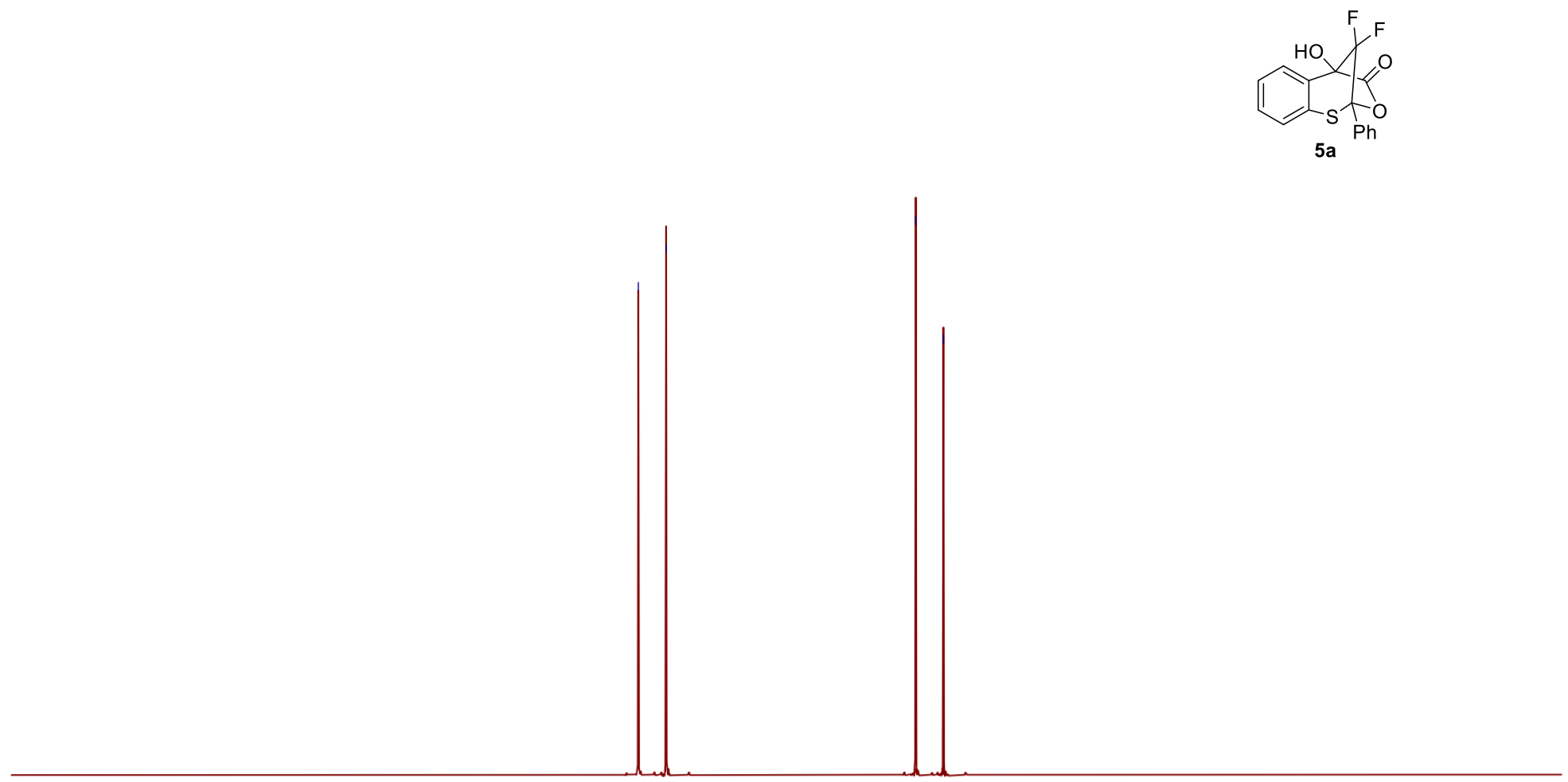

$\begin{array}{lllllllllllllllllllllllllllllllllllllllll}-115 & -116 & -117 & -118 & -119 & -120 & -121 & -122 & -123 & -124 & -125 & -126 & -127 & -128 & -129 & -130 & -131 & -132 & -13 & -134 & -135 & -136 & -137 & -138 & -139 & -140 & -141 & -142 & -143 & -144 & -145\end{array}$

${ }^{19} \mathrm{~F}$ NMR spectra of $\mathbf{5 a}$ in $\mathrm{CDCl}_{3}(376 \mathrm{MHz})$ 

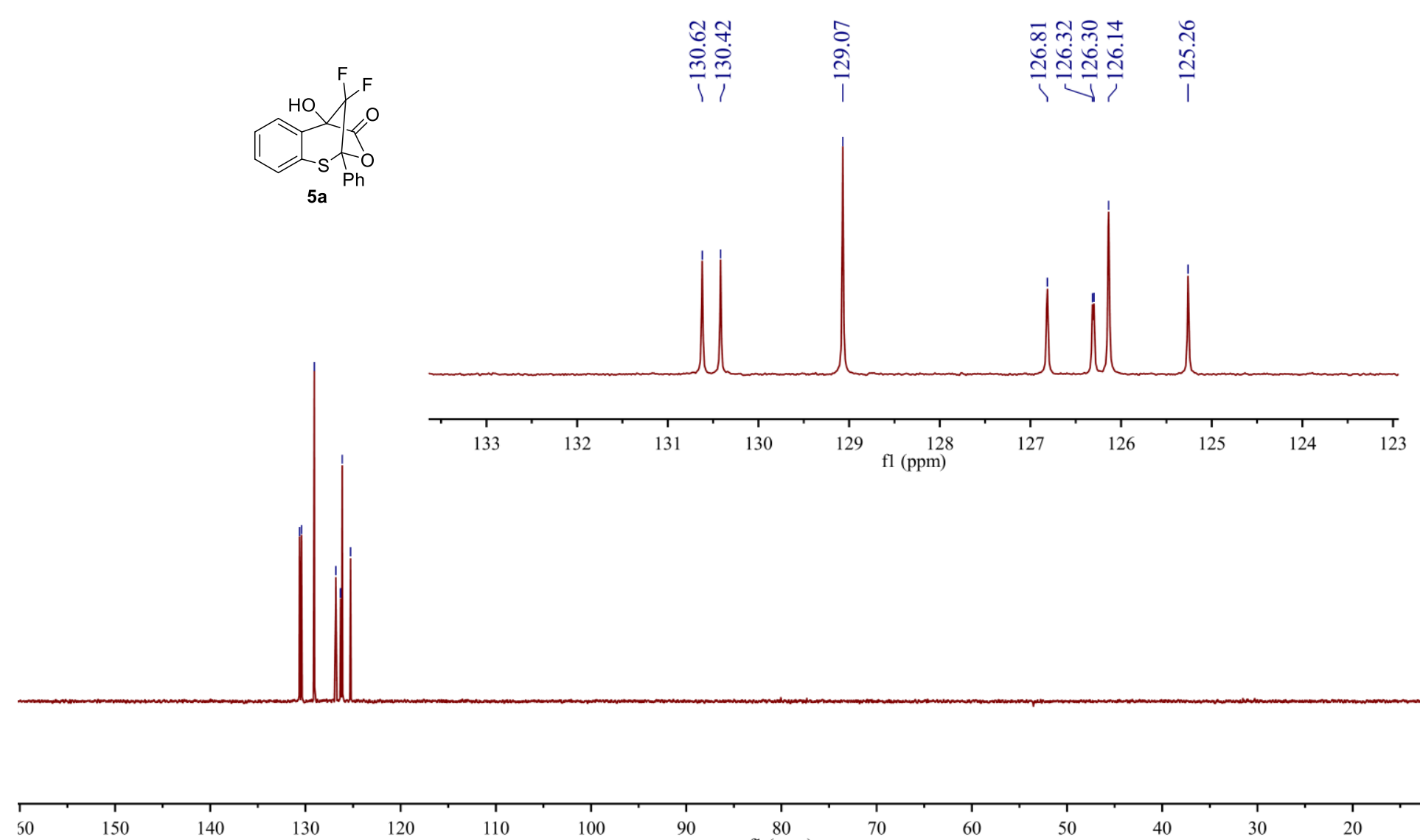

120
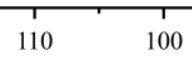

90

80
fl $(\mathrm{ppm})$

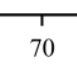

60

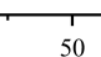

40

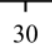

20

DEPT135 of $\mathbf{5 a}$ in $\mathrm{CDCl}_{3}(100 \mathrm{MHz})$ 


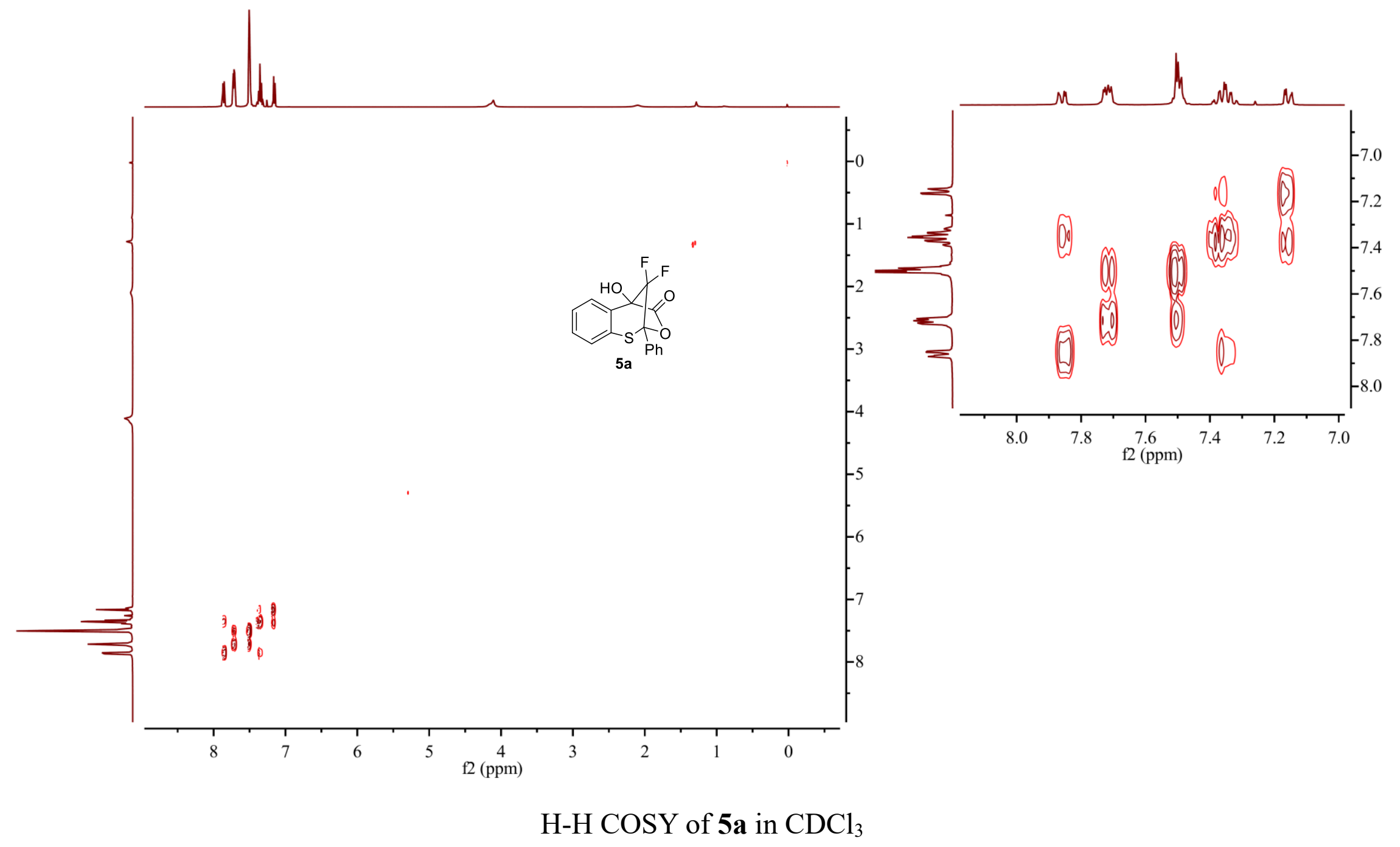




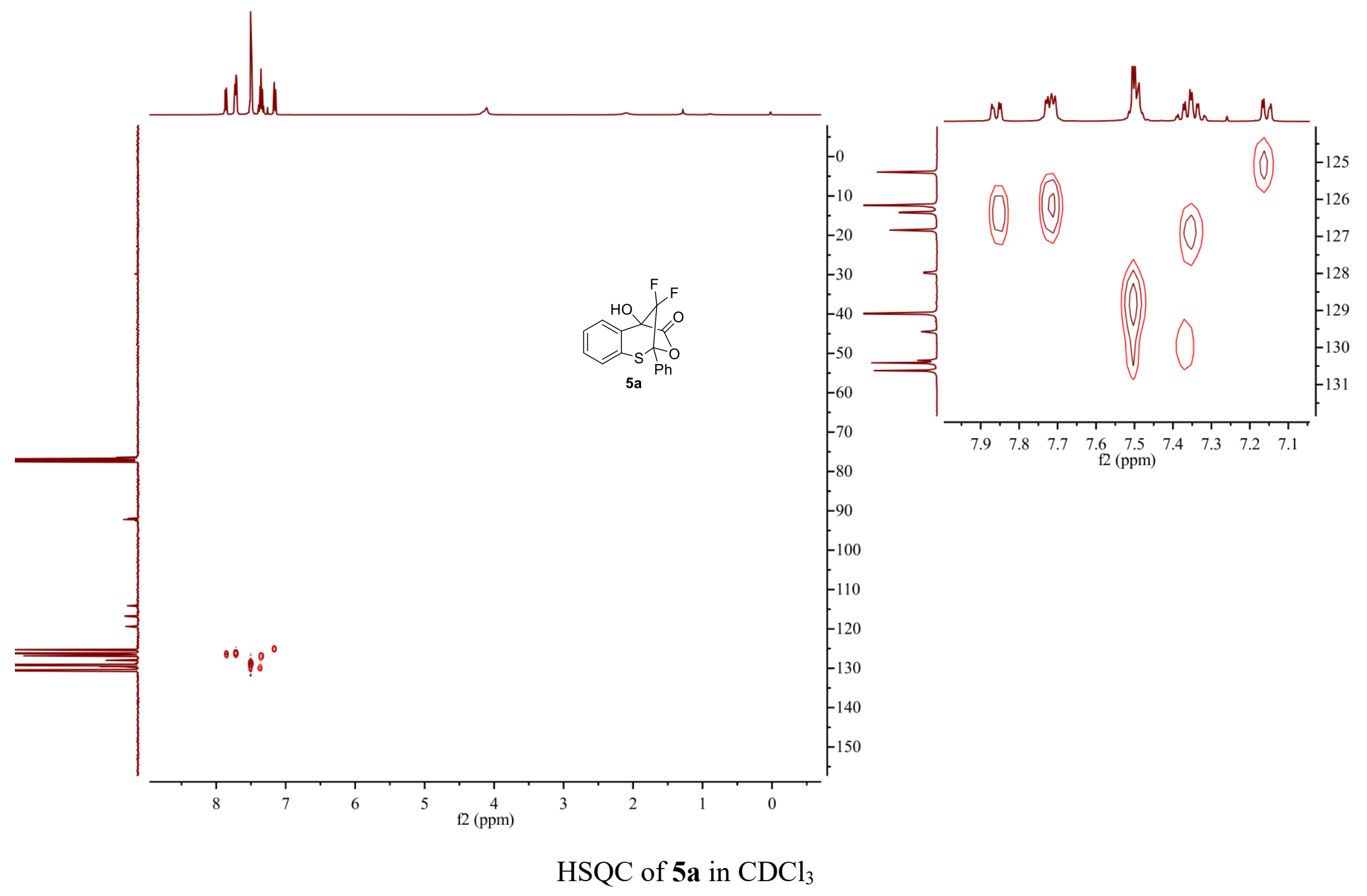




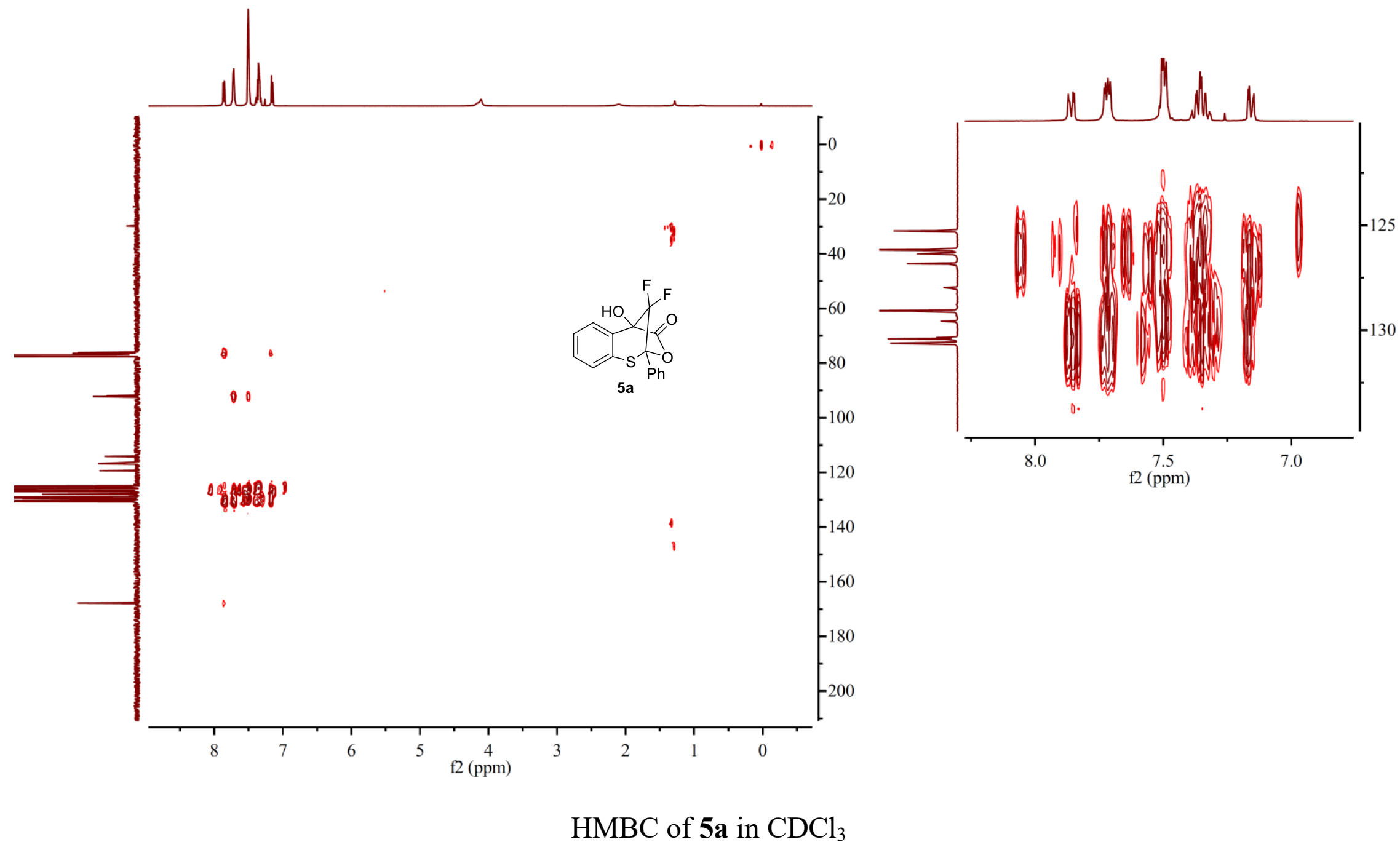




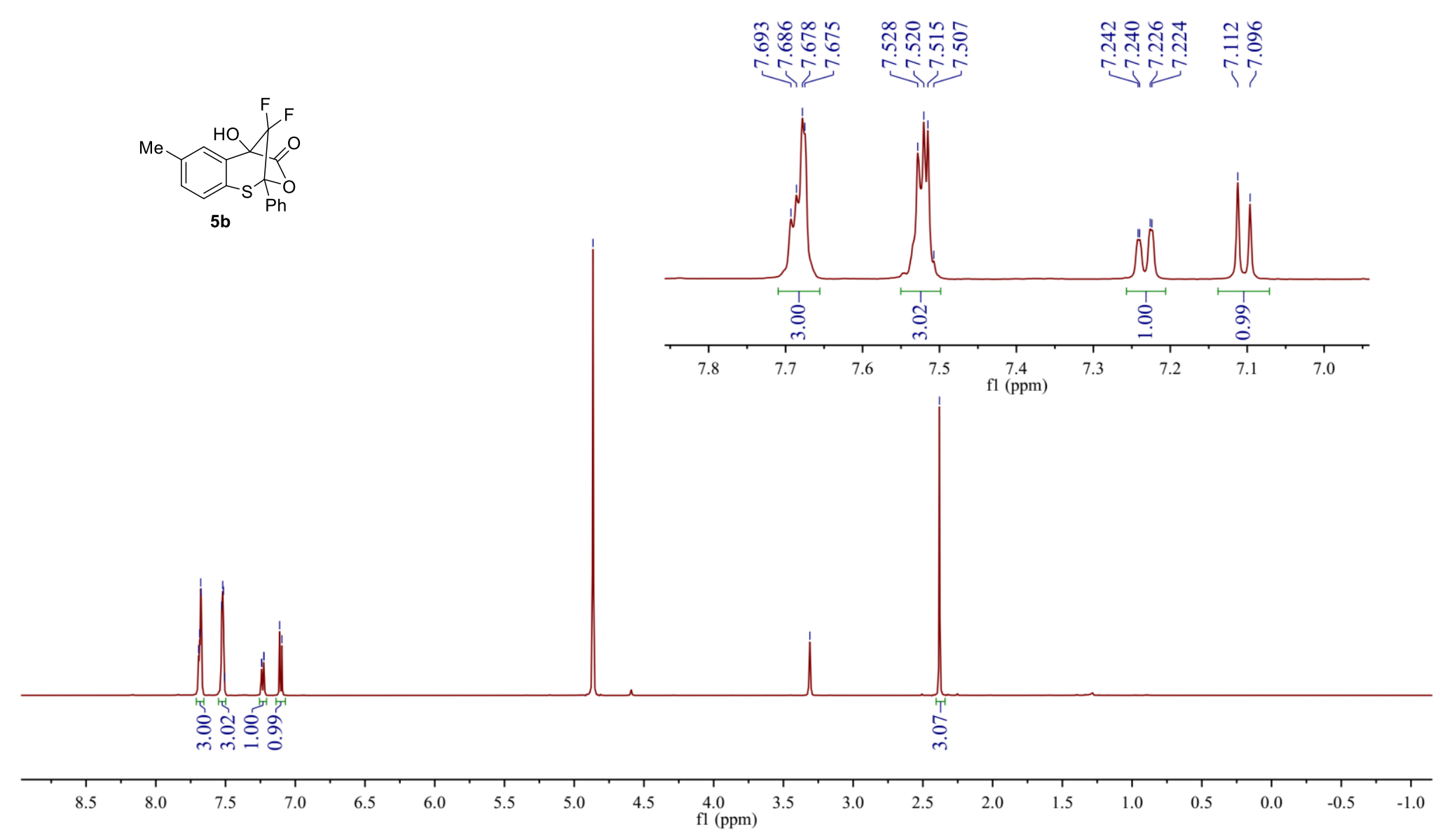

${ }^{1} \mathrm{H}$ NMR spectra of $\mathbf{5 b}$ in $\mathrm{CD}_{3} \mathrm{OD}(500 \mathrm{MHz})$ 

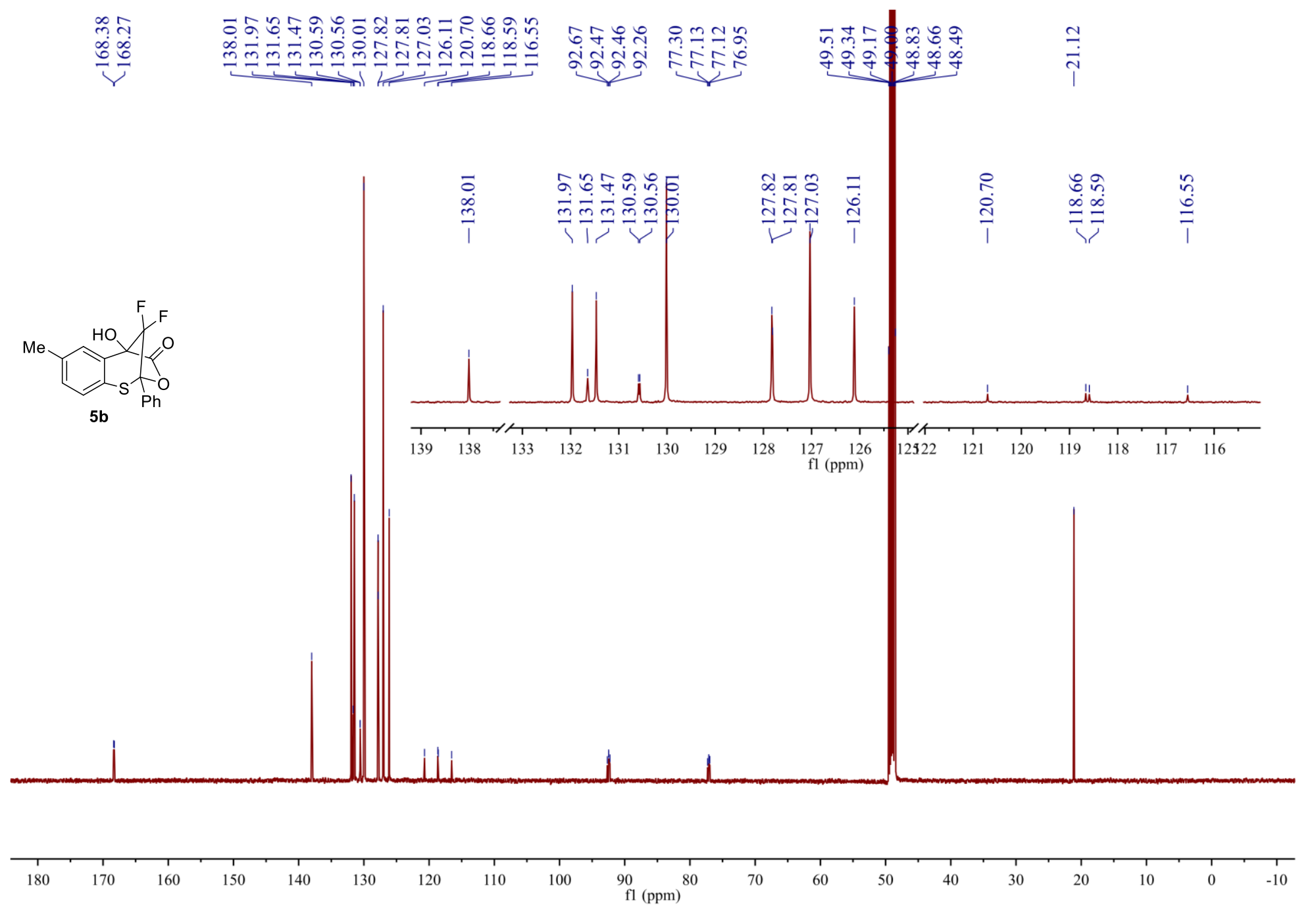

${ }^{19} \mathrm{C}\left\{{ }^{1} \mathrm{H}\right\}$ NMR spectra of $\mathbf{5 b}$ in $\mathrm{CD}_{3} \mathrm{OD}(125 \mathrm{MHz})$ 


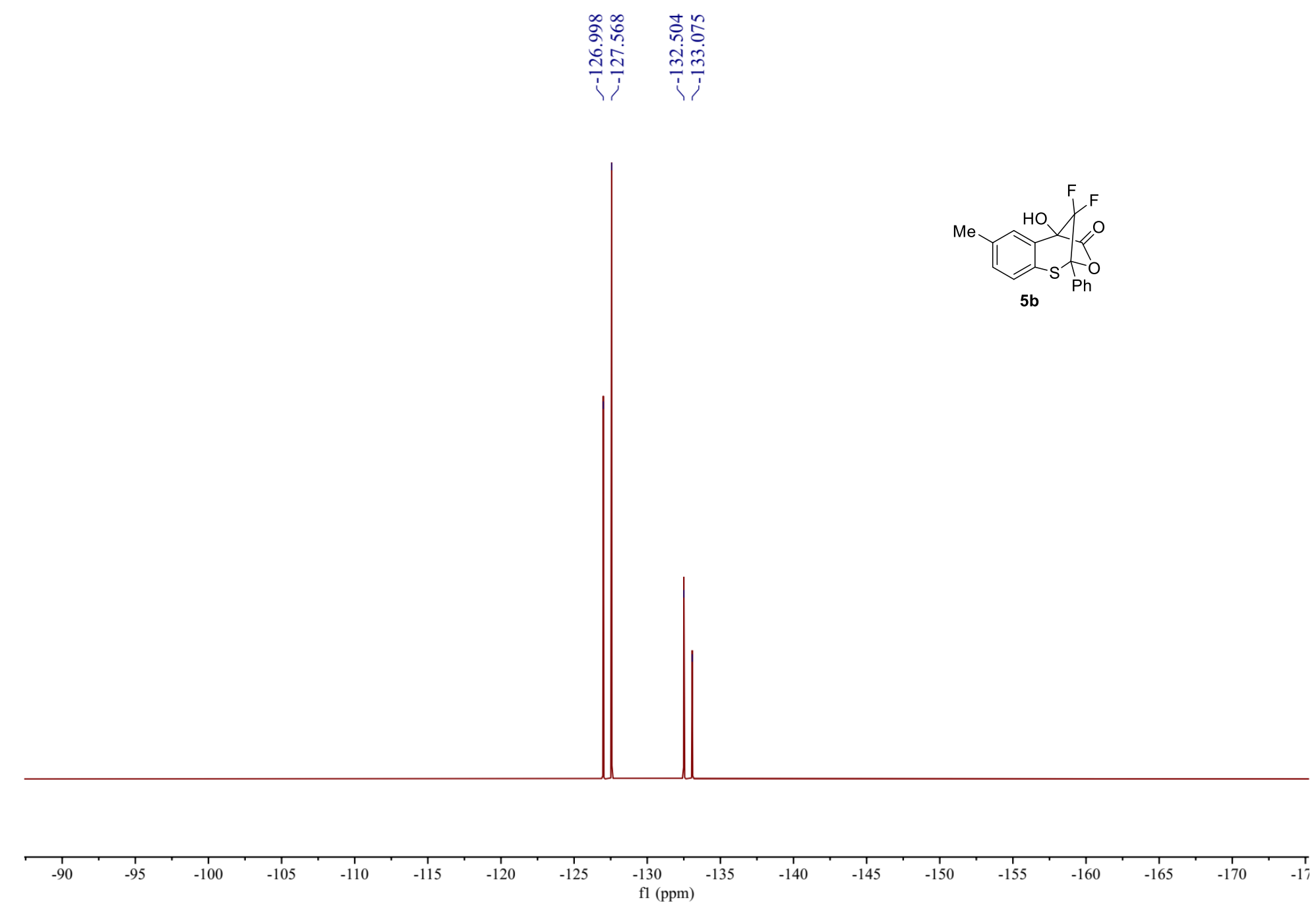

${ }^{19} \mathrm{~F}$ NMR spectra of $\mathbf{5 b}$ in $\mathrm{CDCl}_{3}(376 \mathrm{MHz})$ 


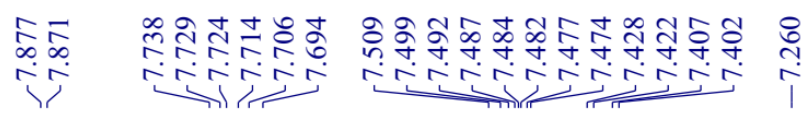

说高
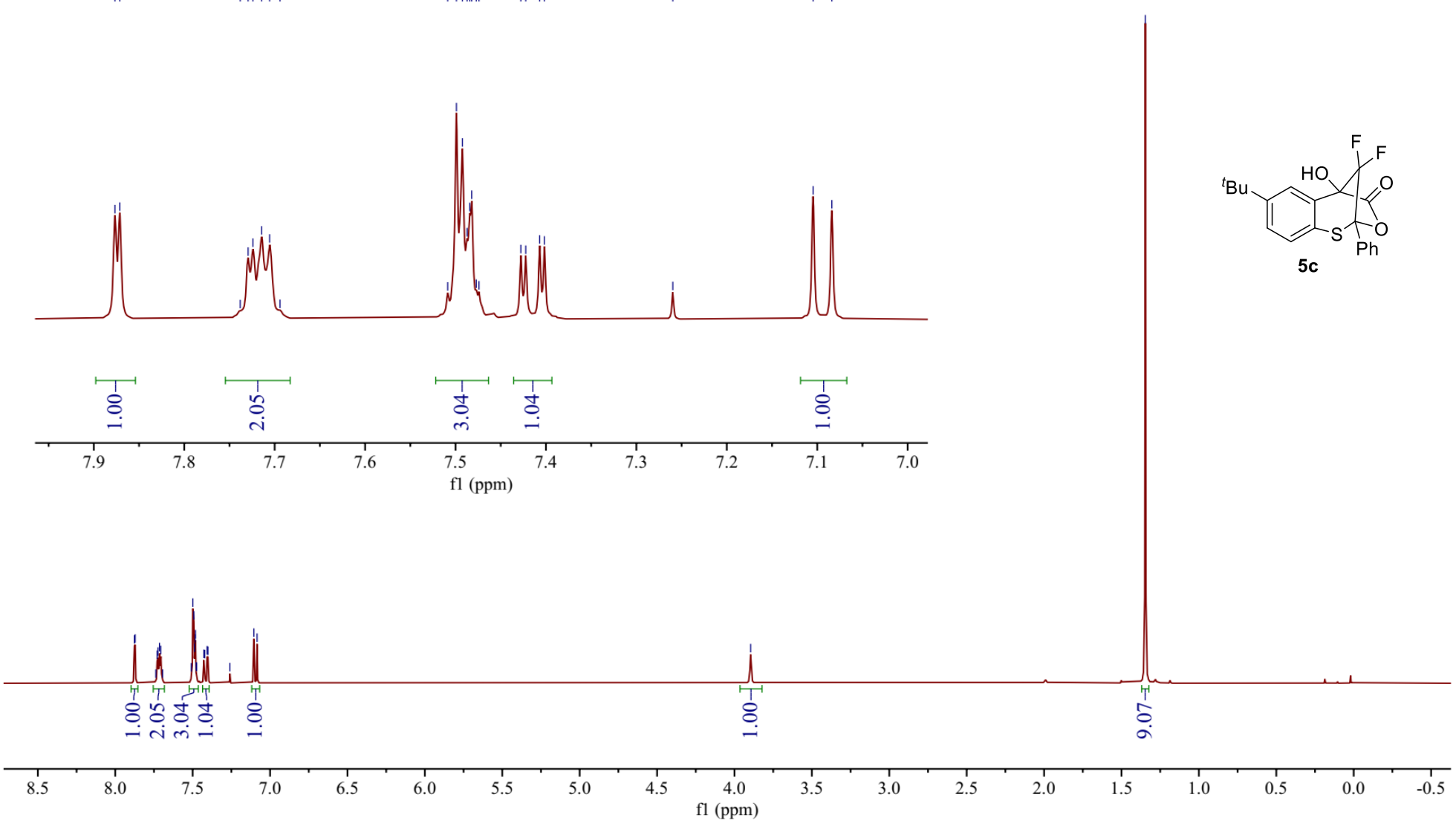

${ }^{1} \mathrm{H}$ NMR spectra of $\mathbf{5}$ in $\mathrm{CDCl}_{3}(400 \mathrm{MHz})$ 

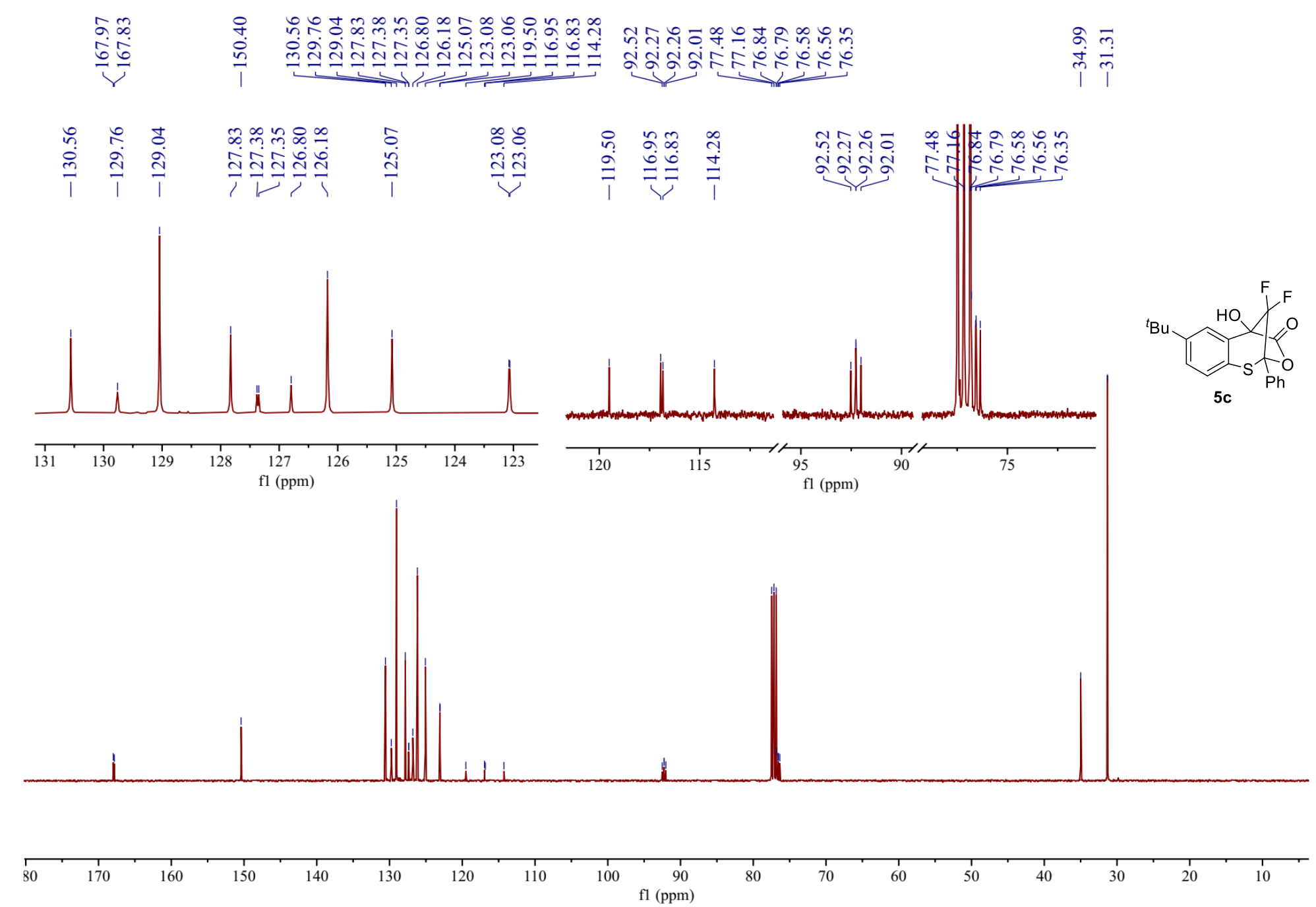

${ }^{13} \mathrm{C}\left\{{ }^{1} \mathrm{H}\right\}$ NMR spectra of $\mathbf{5 c}$ in $\mathrm{CDCl}_{3}(100 \mathrm{MHz})$ 


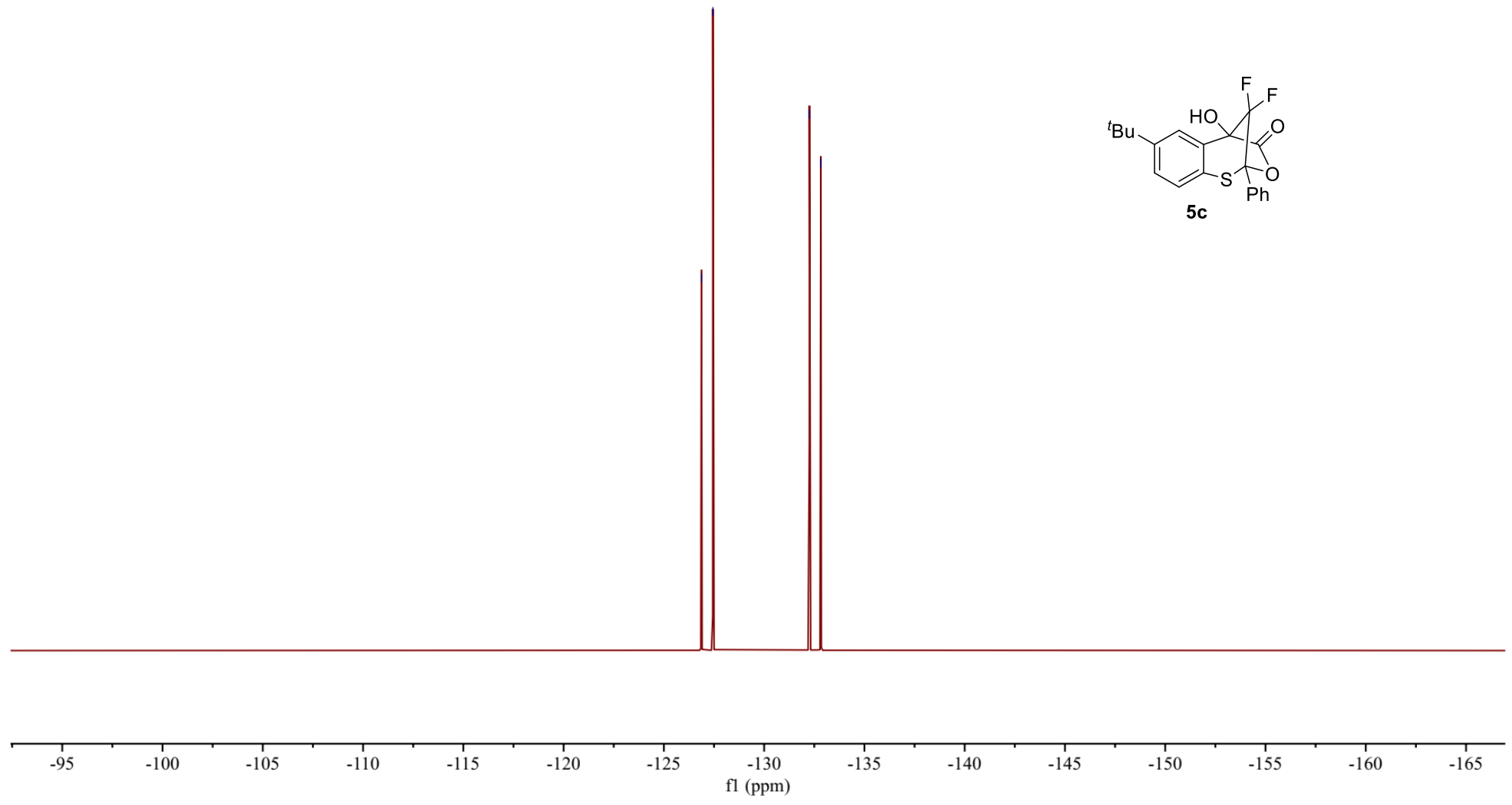

${ }^{19} \mathrm{~F}$ NMR spectra of $\mathbf{5 c}$ in $\mathrm{CDCl}_{3}(376 \mathrm{MHz})$ 


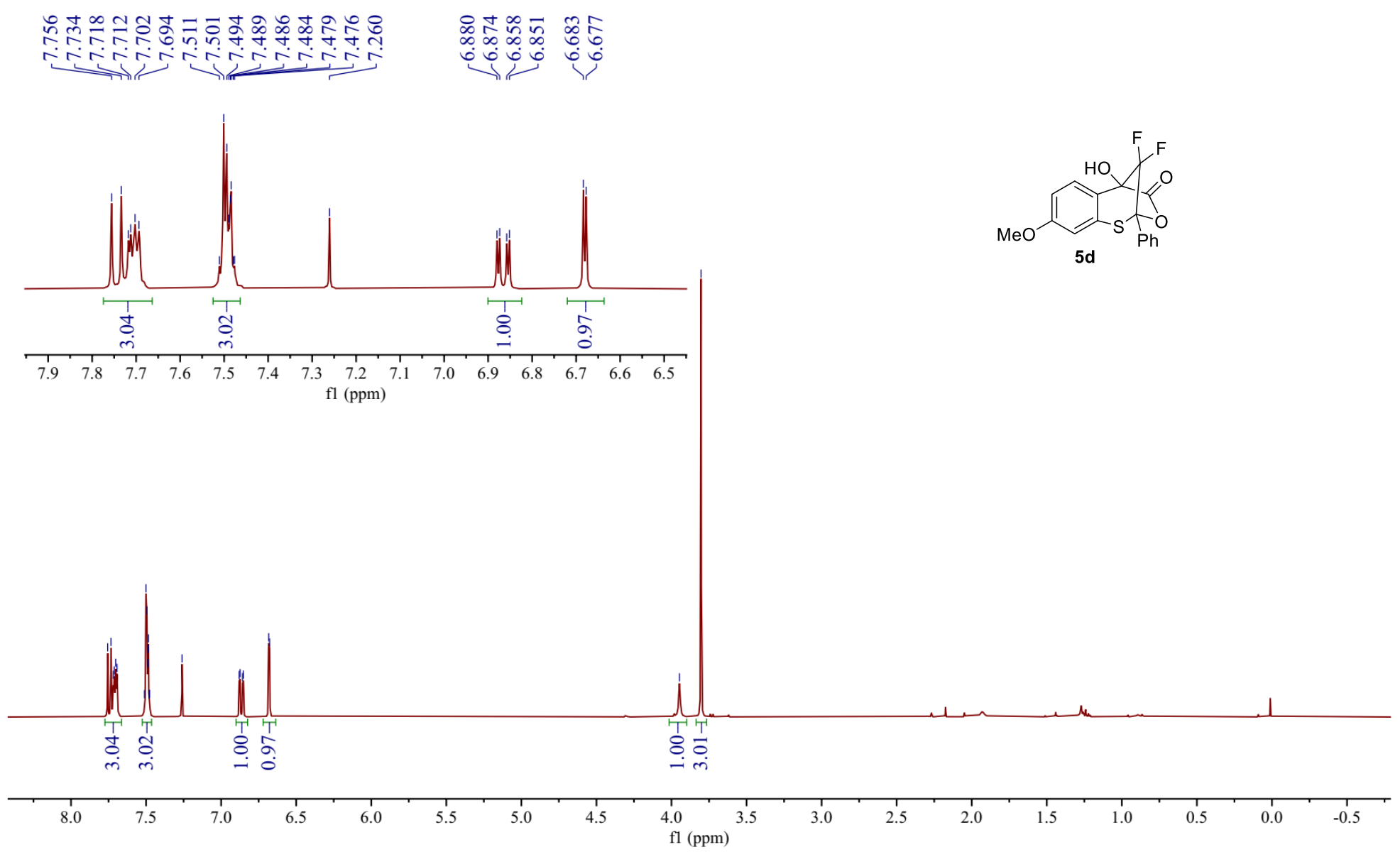

${ }^{1} \mathrm{H}$ NMR spectra of $\mathbf{5 d}$ in $\mathrm{CDCl}_{3}(400 \mathrm{MHz})$ 

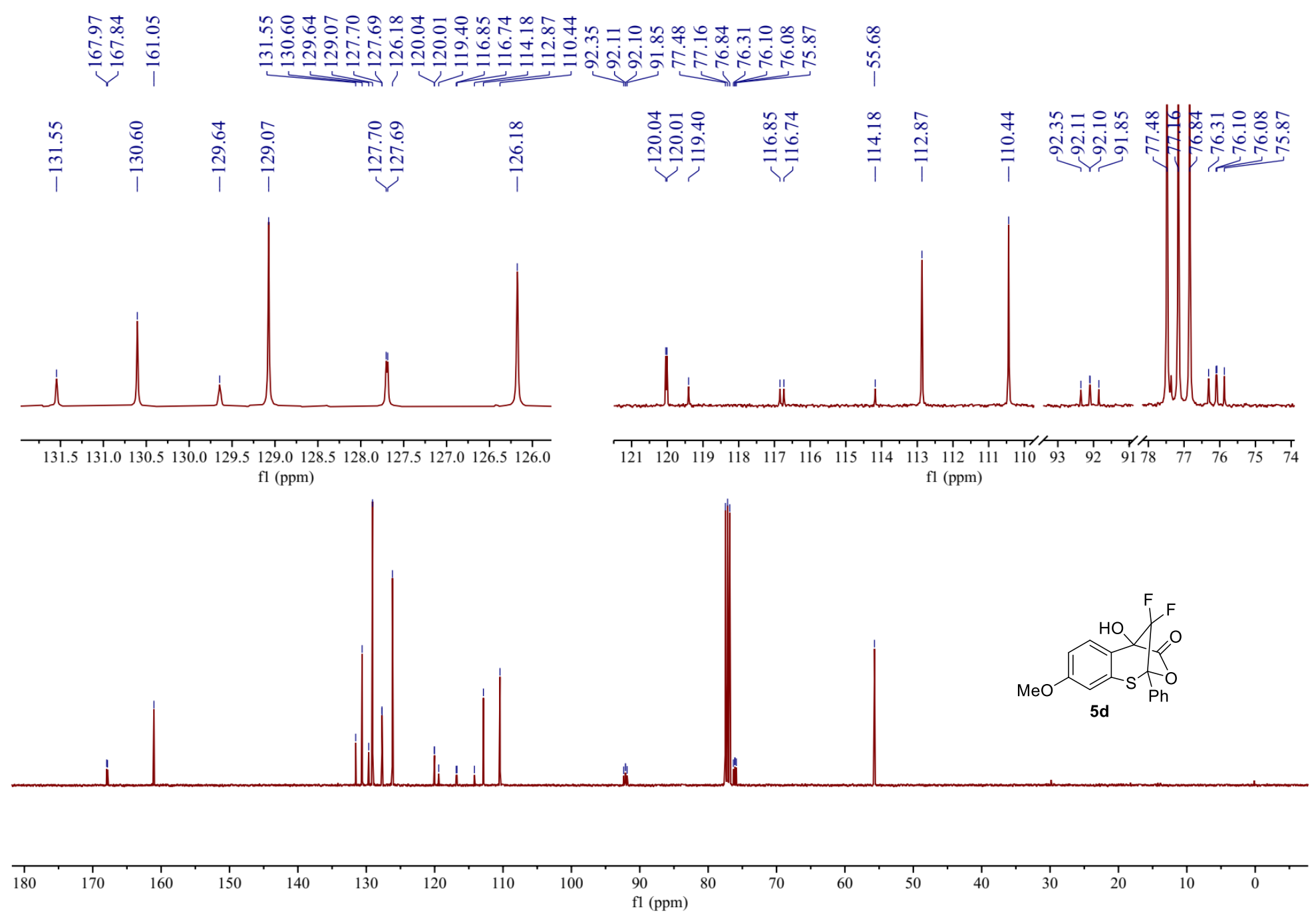

${ }^{13} \mathrm{C}\left\{{ }^{1} \mathrm{H}\right\}$ NMR spectra of $\mathbf{5 d}$ in $\mathrm{CDCl}_{3}(100 \mathrm{MHz})$ 


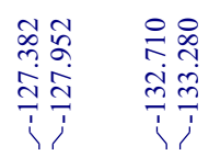
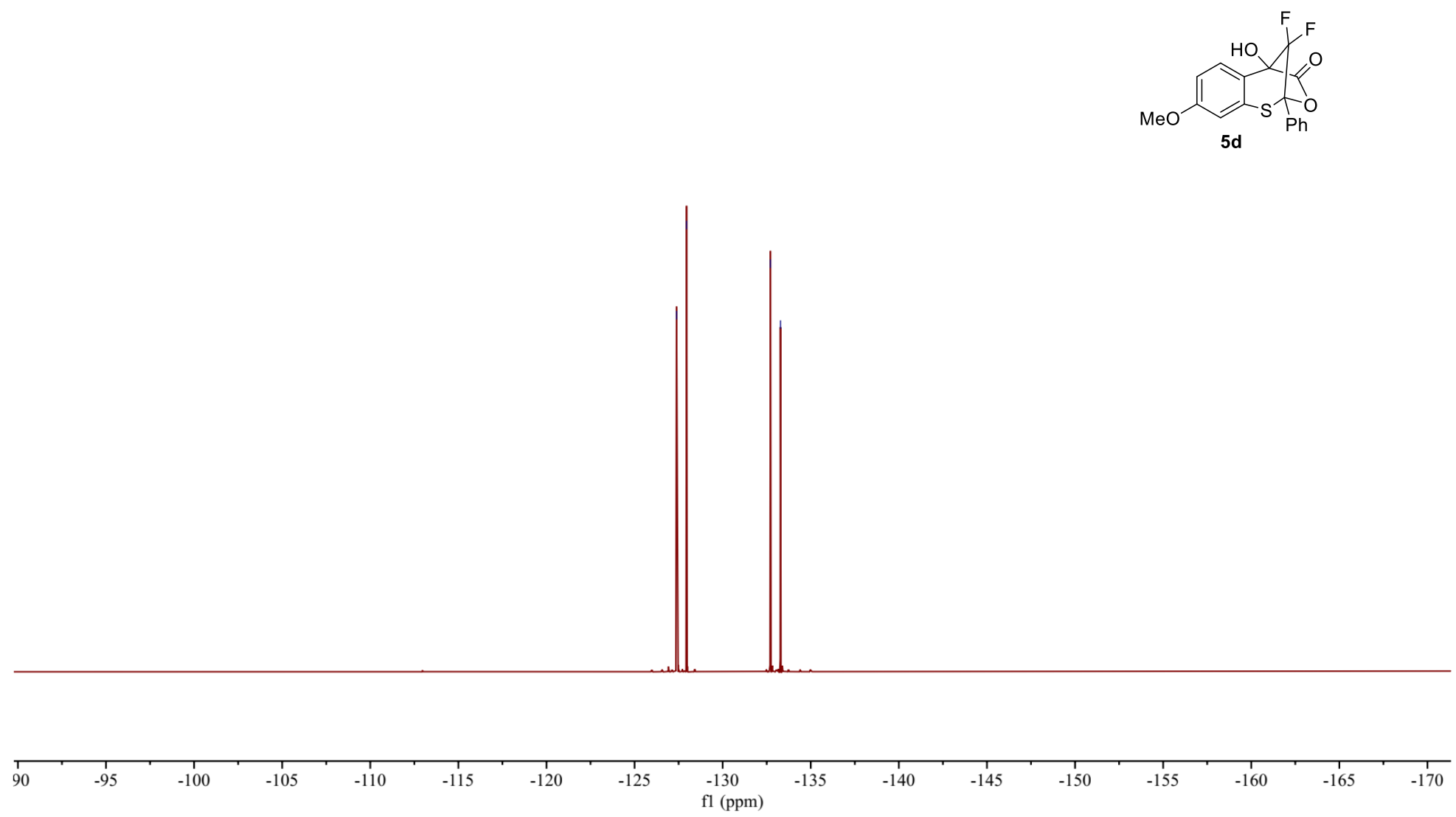

${ }^{19} \mathrm{~F}$ NMR spectra of $\mathbf{5 d}$ in $\mathrm{CDCl}_{3}(376 \mathrm{MHz})$ 


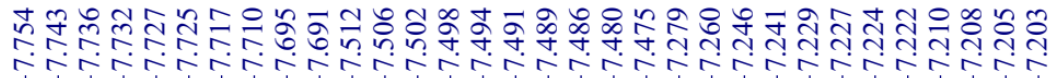
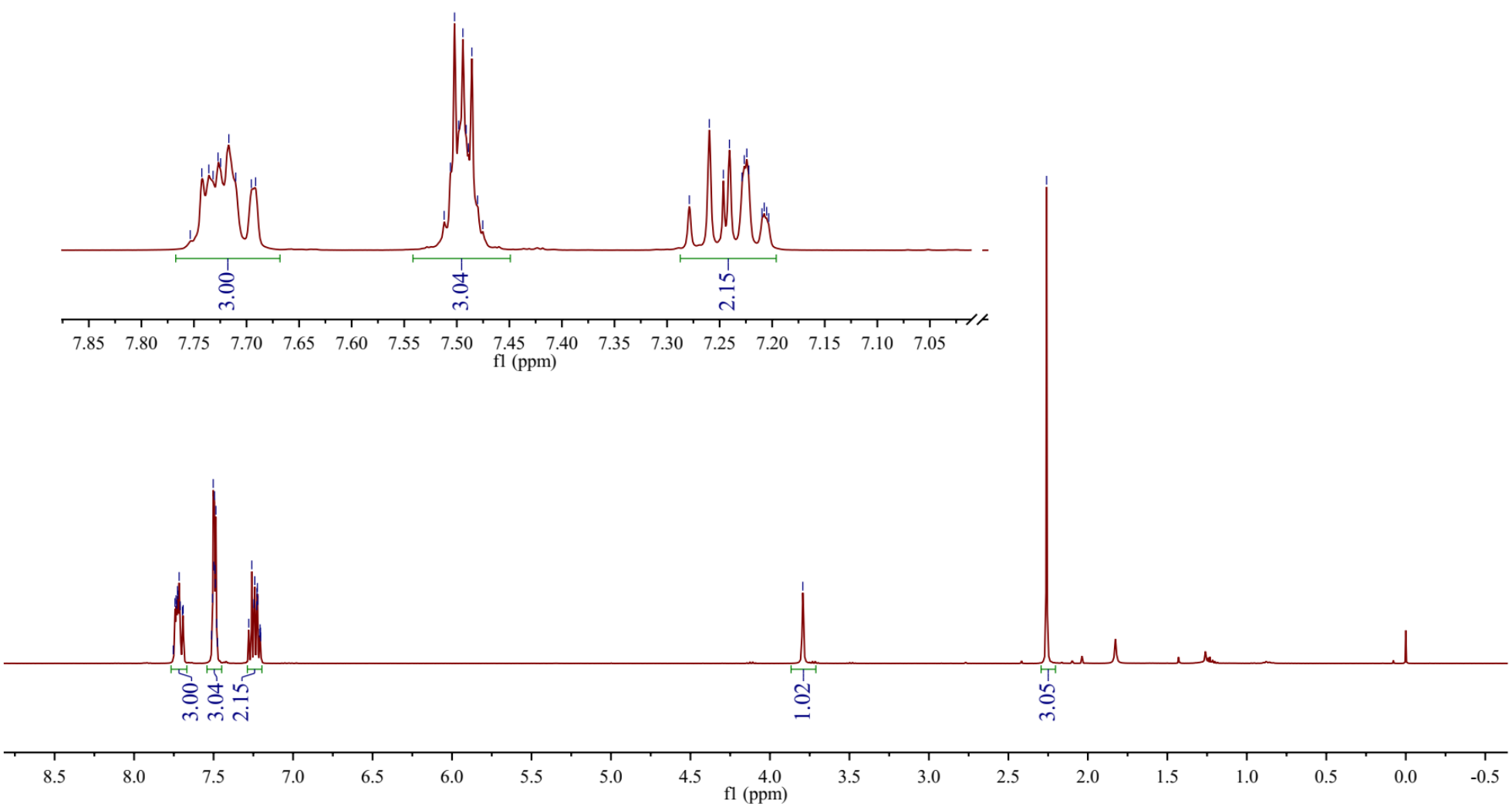

${ }^{1} \mathrm{H}$ NMR spectra of $\mathbf{5 e}$ in $\mathrm{CDCl}_{3}(400 \mathrm{MHz})$ 

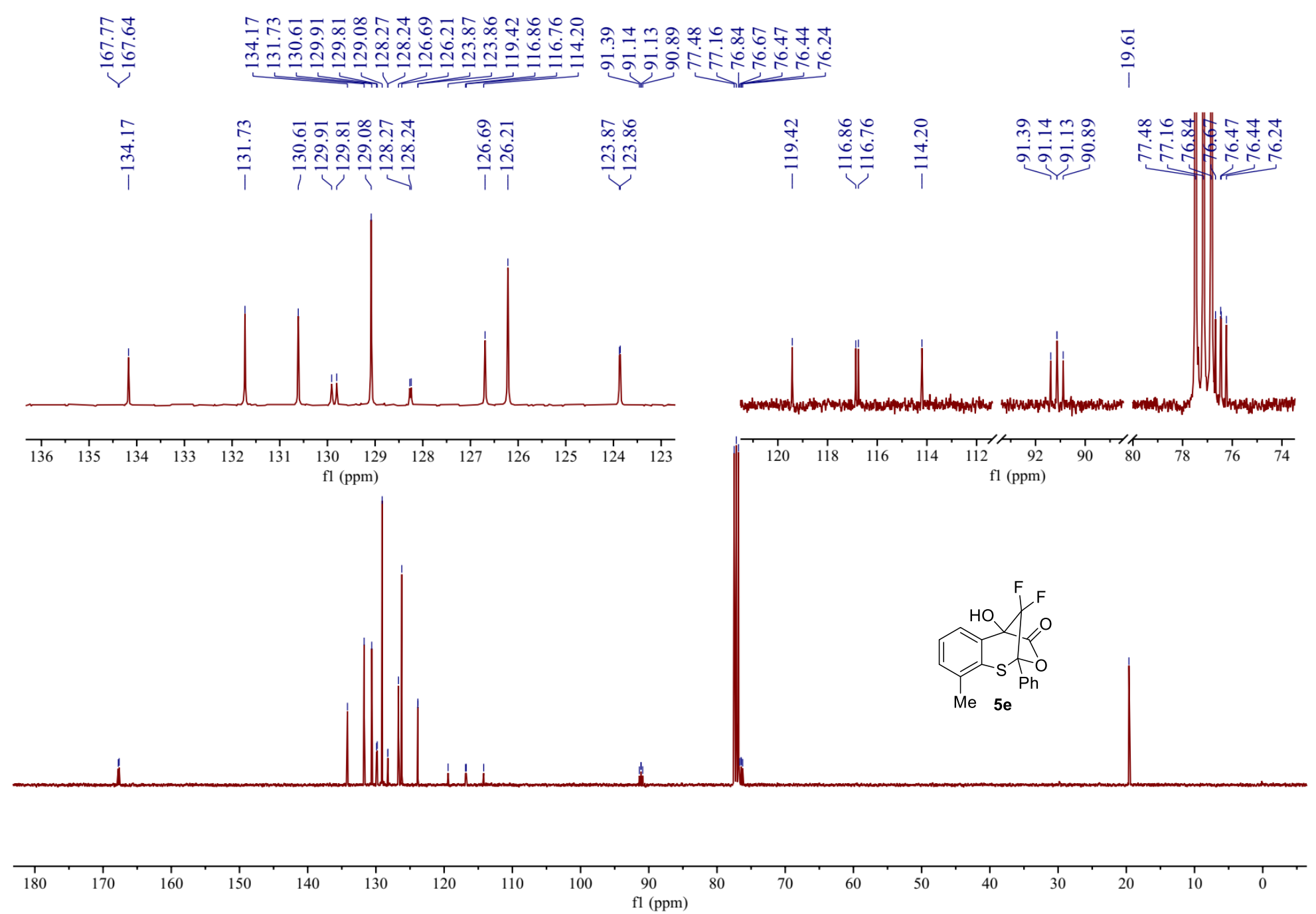

${ }^{13} \mathrm{C}\left\{{ }^{1} \mathrm{H}\right\}$ NMR spectra of $\mathbf{5 e}$ in $\mathrm{CDCl}_{3}(100 \mathrm{MHz})$ 


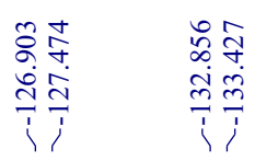

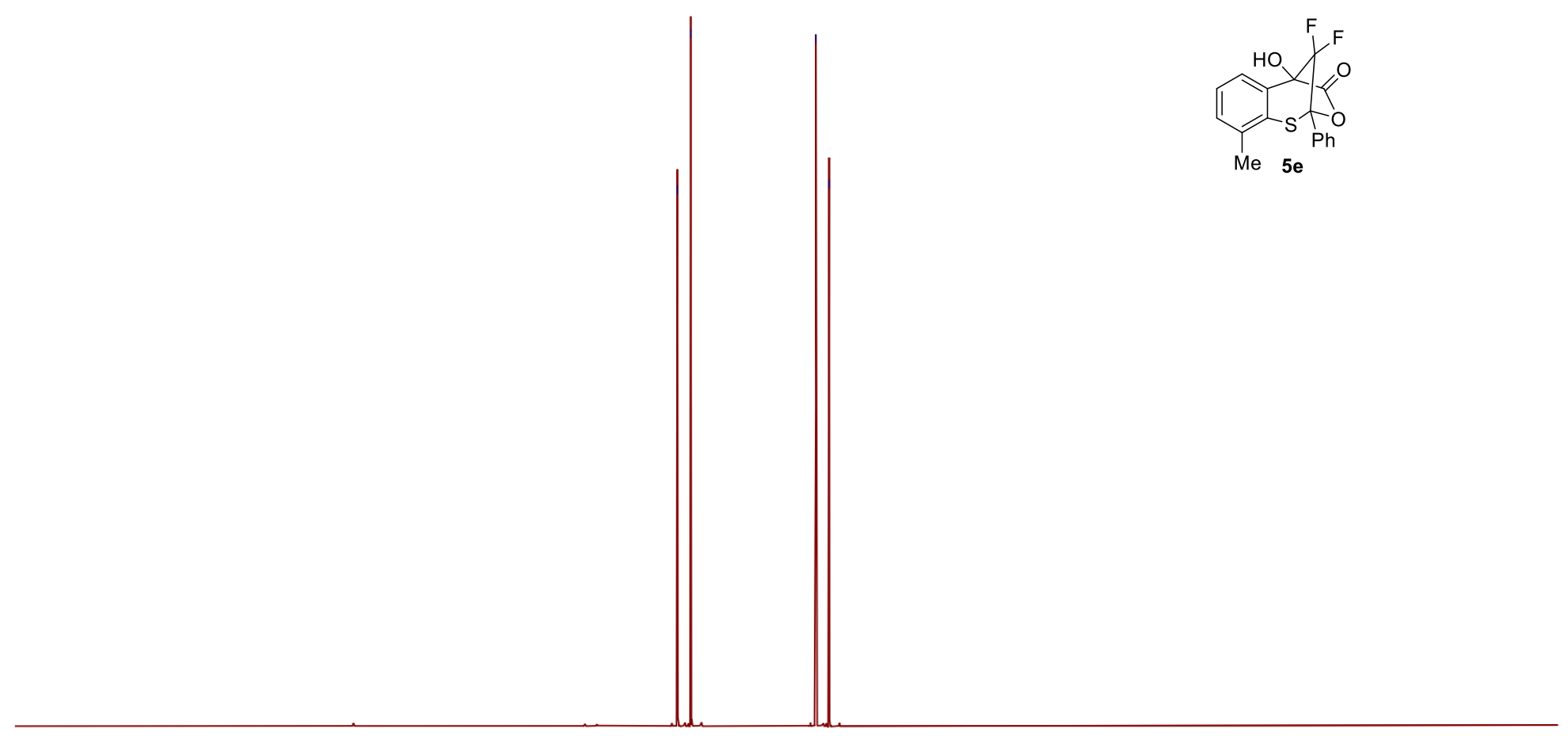

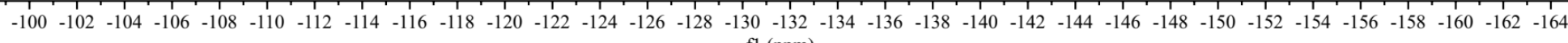
fl (ppm)

${ }^{19} \mathrm{~F}$ NMR spectra of $\mathbf{5 e}$ in $\mathrm{CDCl}_{3}(376 \mathrm{MHz})$ 


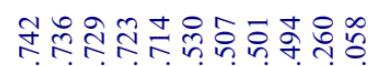

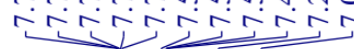

$\underset{\substack{0 \\ i}}{\infty}$

iై

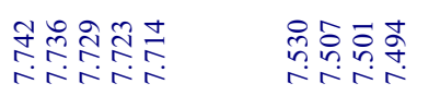

तinit

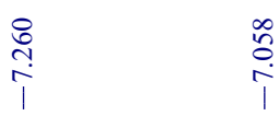
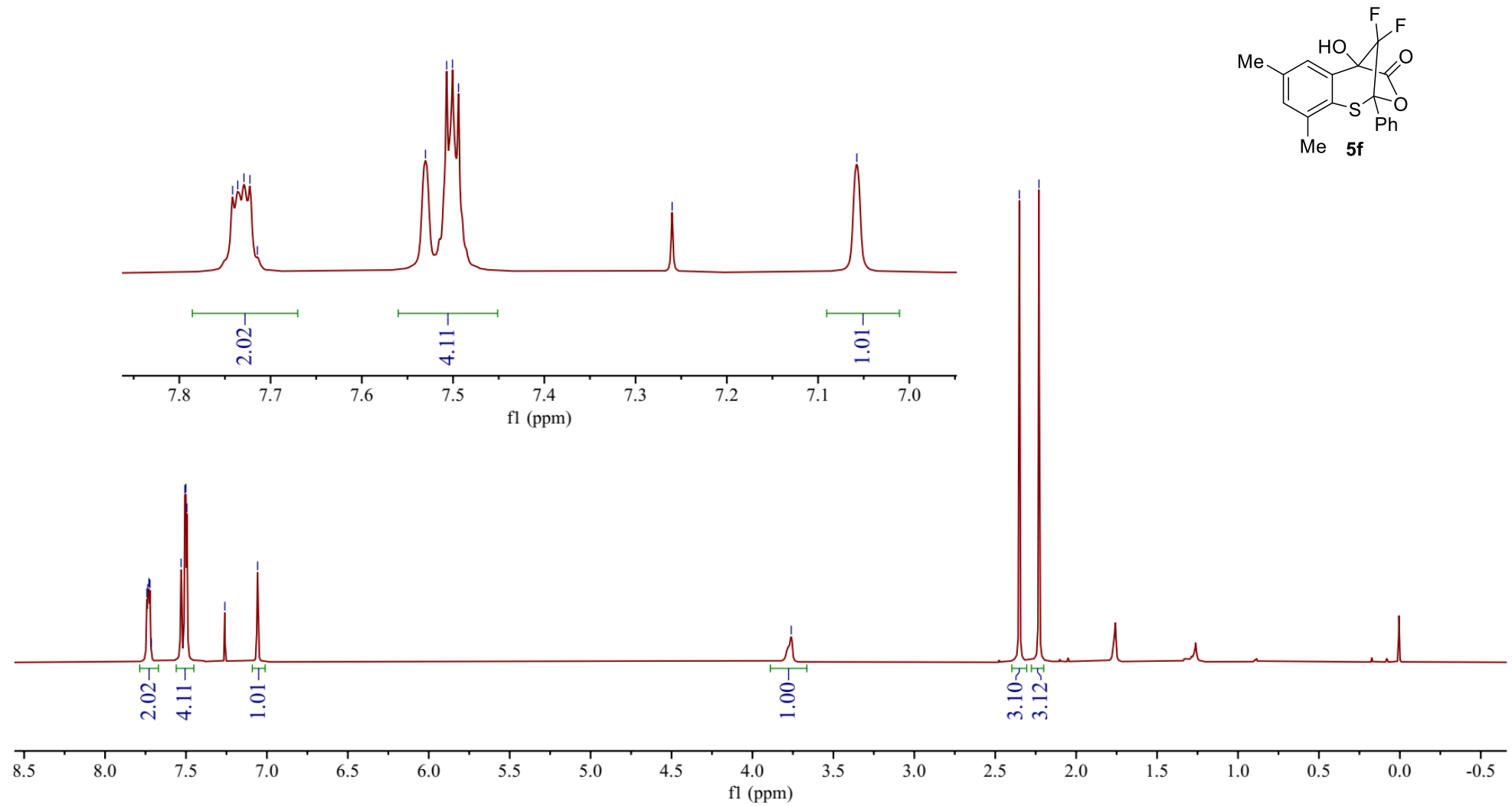

${ }^{1} \mathrm{H}$ NMR spectra of $\mathbf{5 f}$ in $\mathrm{CDCl}_{3}(400 \mathrm{MHz})$ 

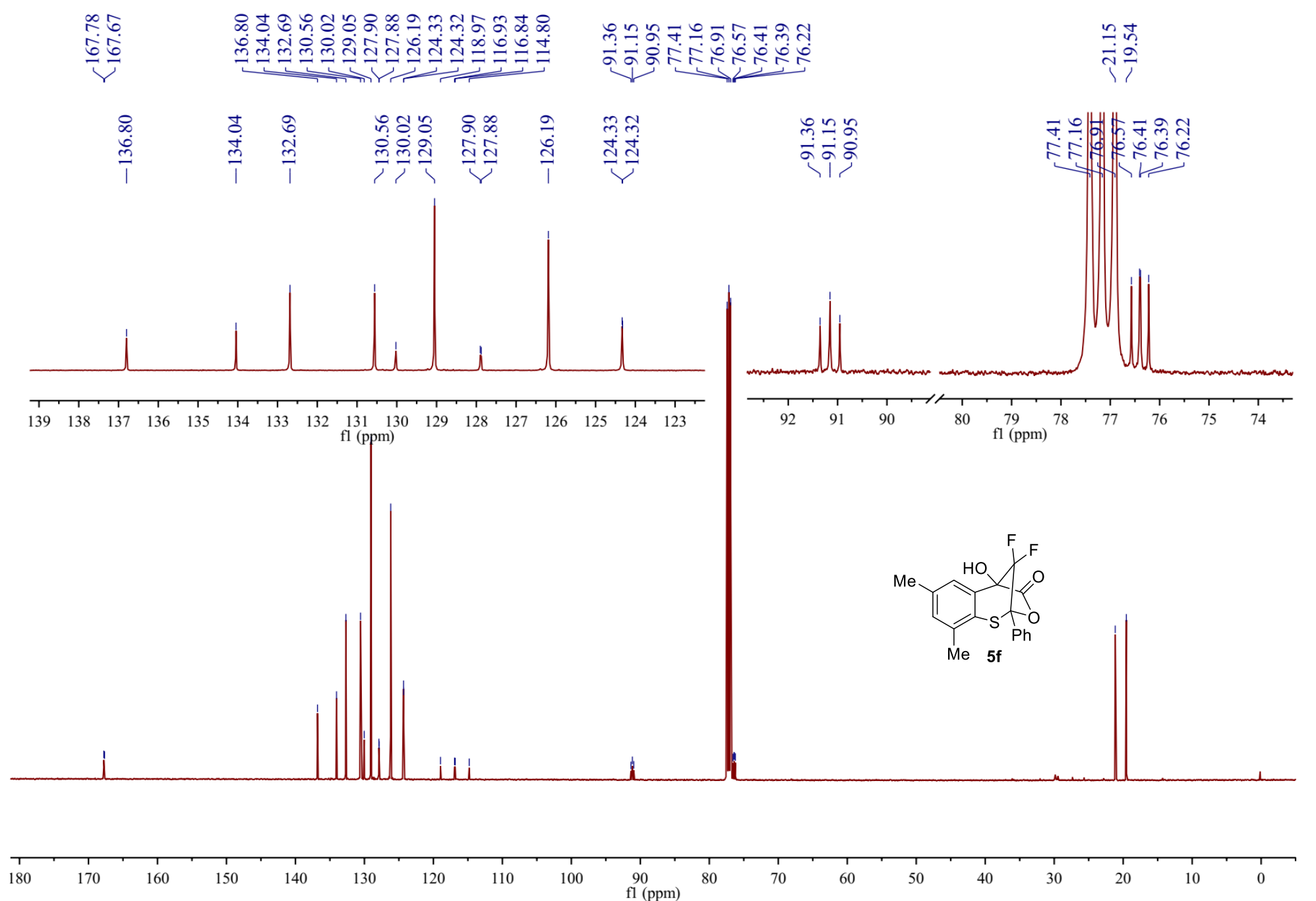

${ }^{13} \mathrm{C}\left\{{ }^{1} \mathrm{H}\right\}$ NMR spectra of $\mathbf{5 f}$ in $\mathrm{CDCl}_{3}(100 \mathrm{MHz})$ 


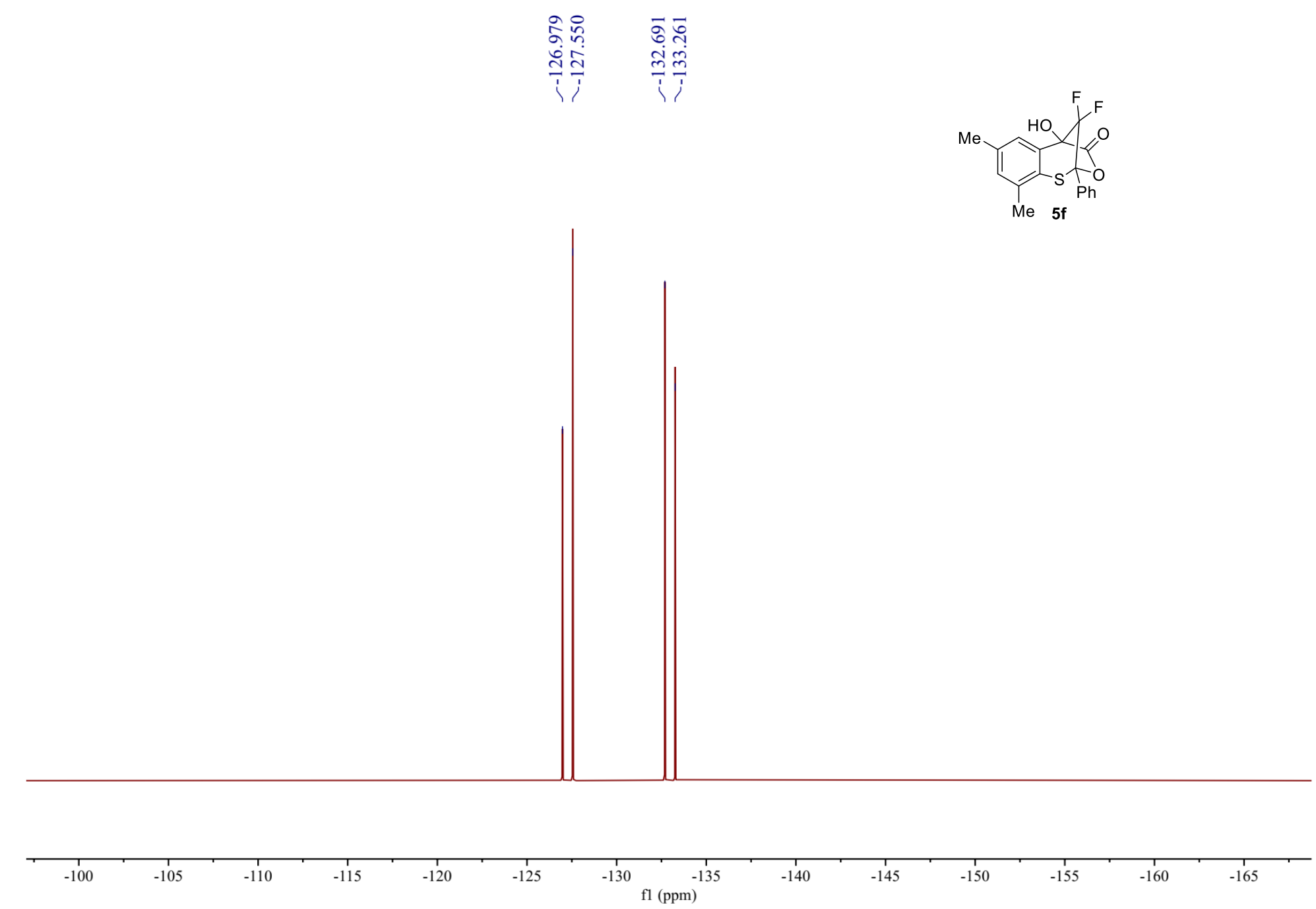

${ }^{19} \mathrm{~F}$ NMR spectra of $\mathbf{5 f}$ in $\mathrm{CDCl}_{3}(376 \mathrm{MHz})$ 


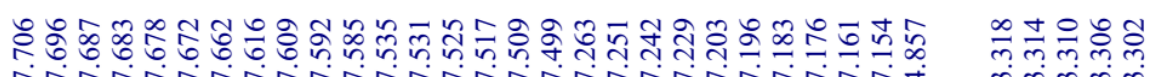

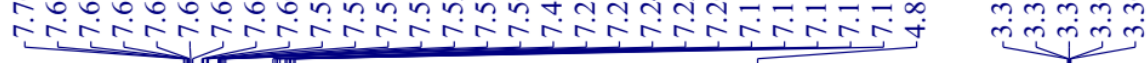

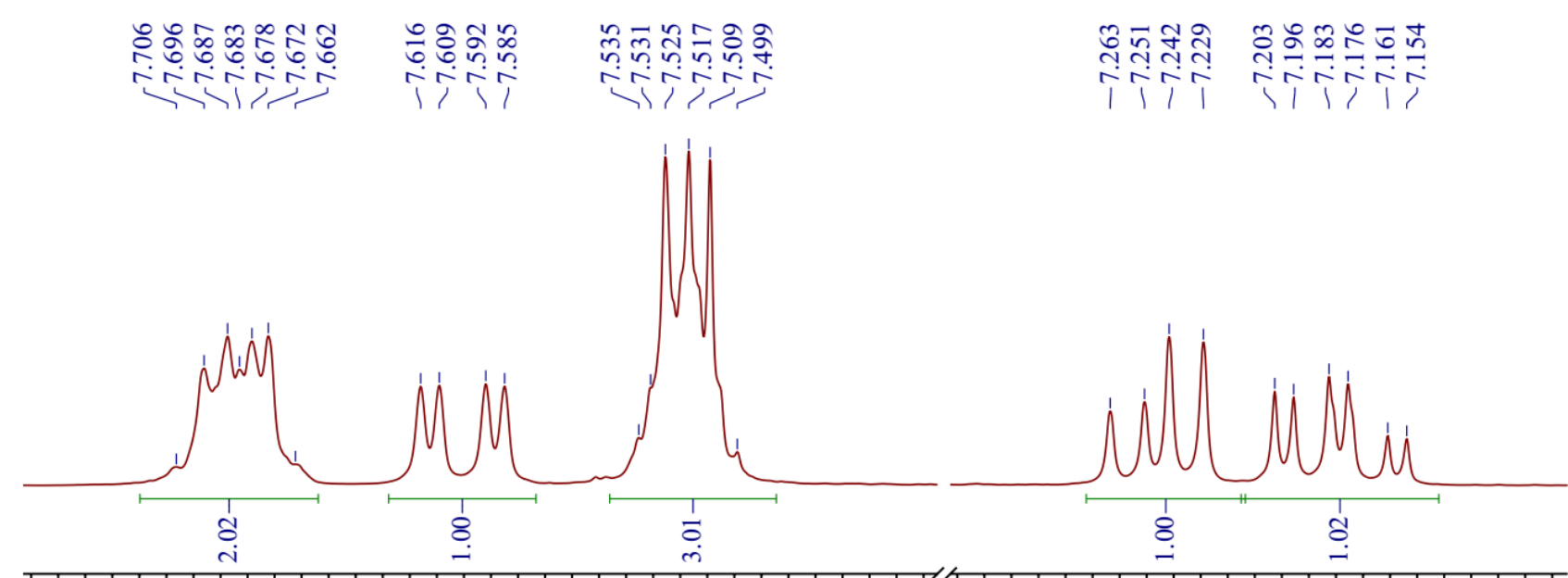

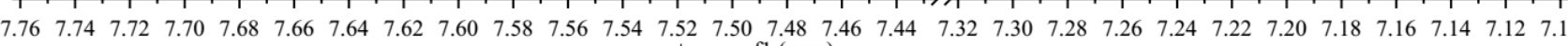
fl $(\mathrm{ppm})$

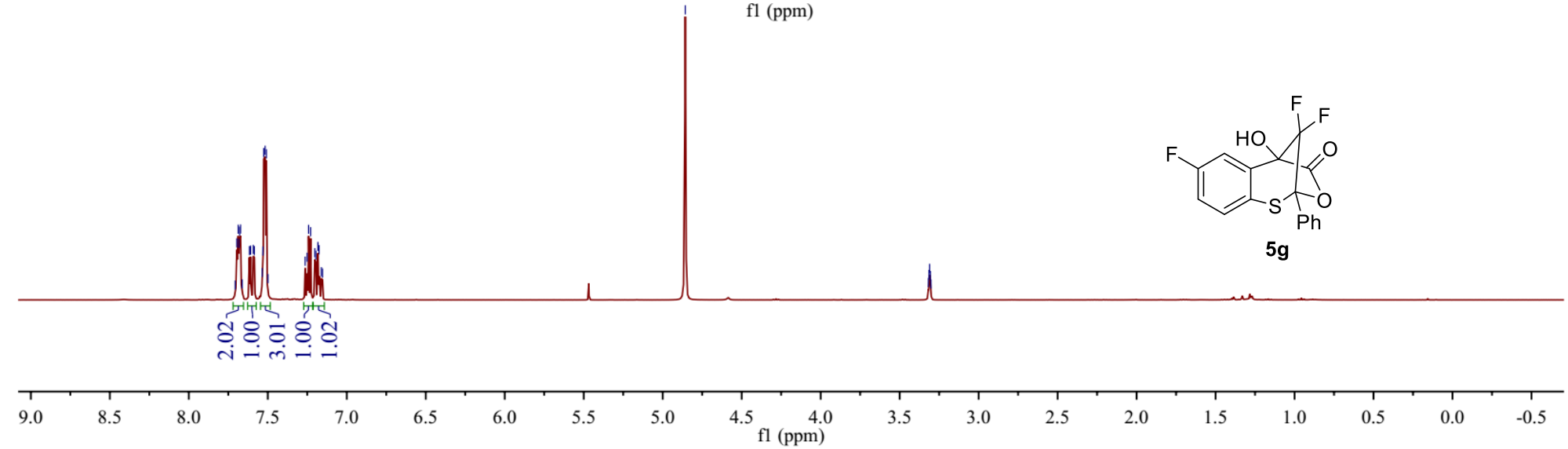

${ }^{1} \mathrm{H}$ NMR spectra of $\mathbf{5 g}$ in $\mathrm{CD}_{3} \mathrm{OD}(400 \mathrm{MHz})$ 

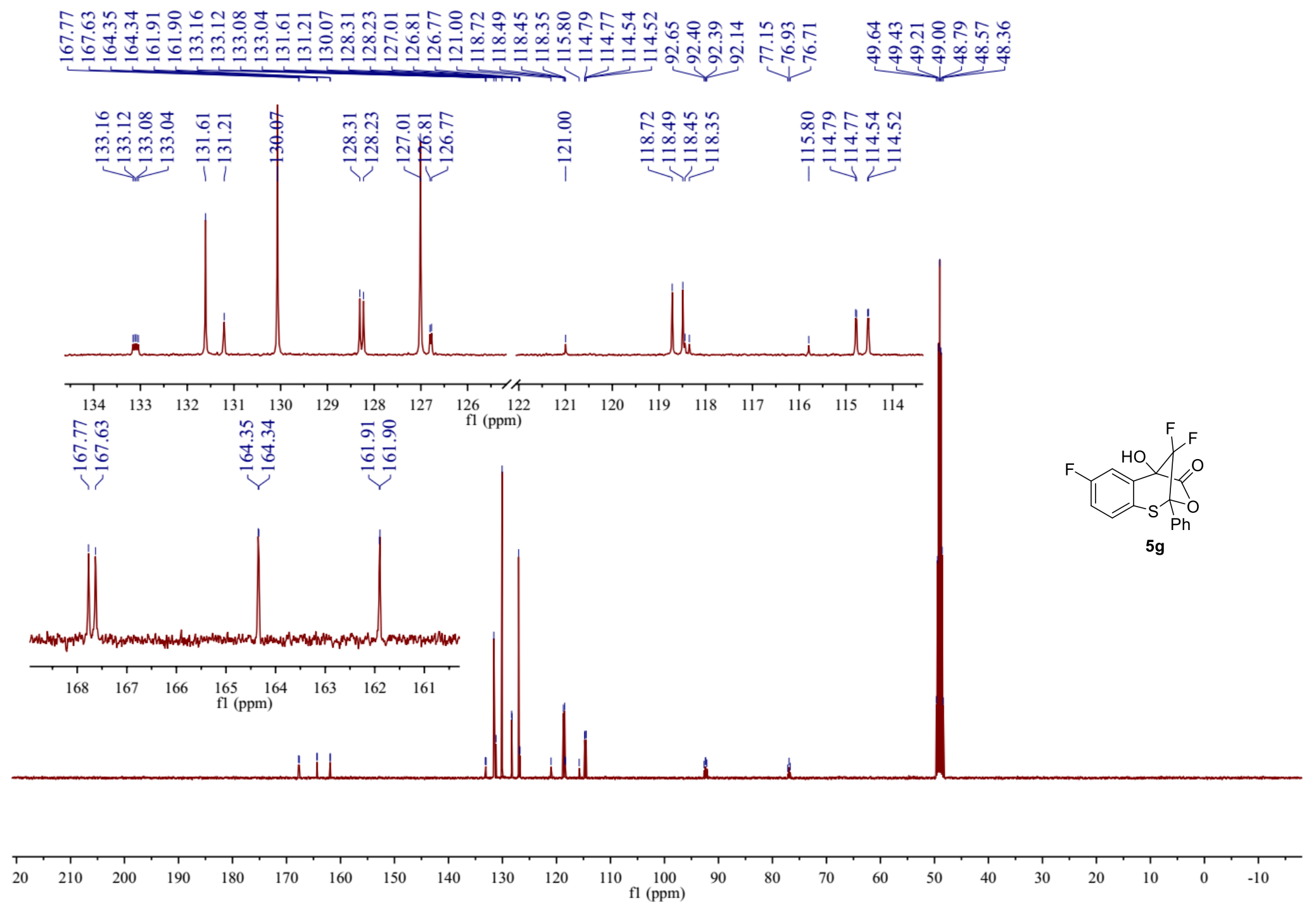

${ }^{13} \mathrm{C}\left\{{ }^{1} \mathrm{H}\right\}$ NMR spectra of $\mathbf{5 g}$ in $\mathrm{CD}_{3} \mathrm{OD}(100 \mathrm{MHz})$ 

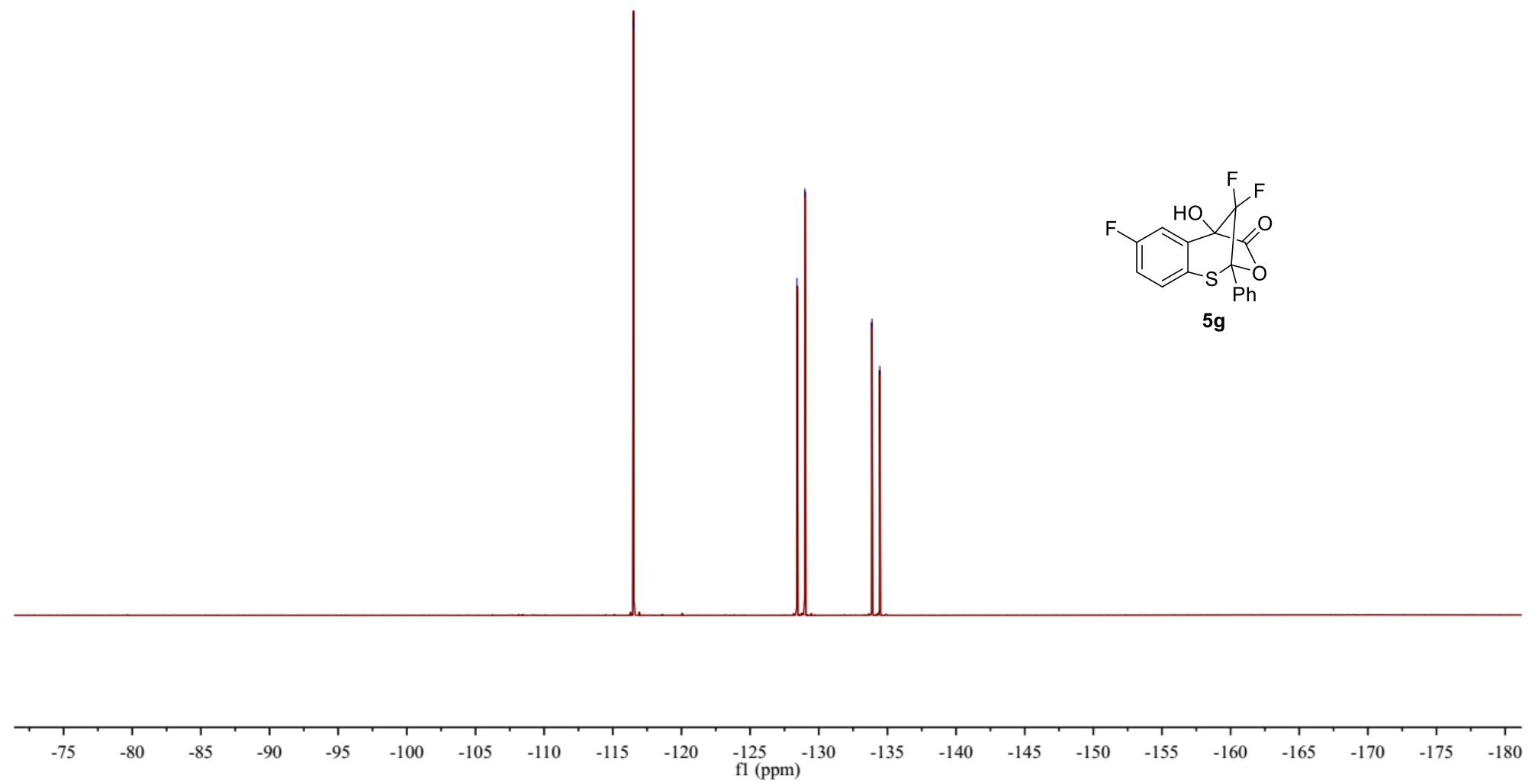

${ }^{19} \mathrm{~F}$ NMR spectra of $\mathbf{5 g}$ in $\mathrm{CD}_{3} \mathrm{OD}(376 \mathrm{MHz})$ 


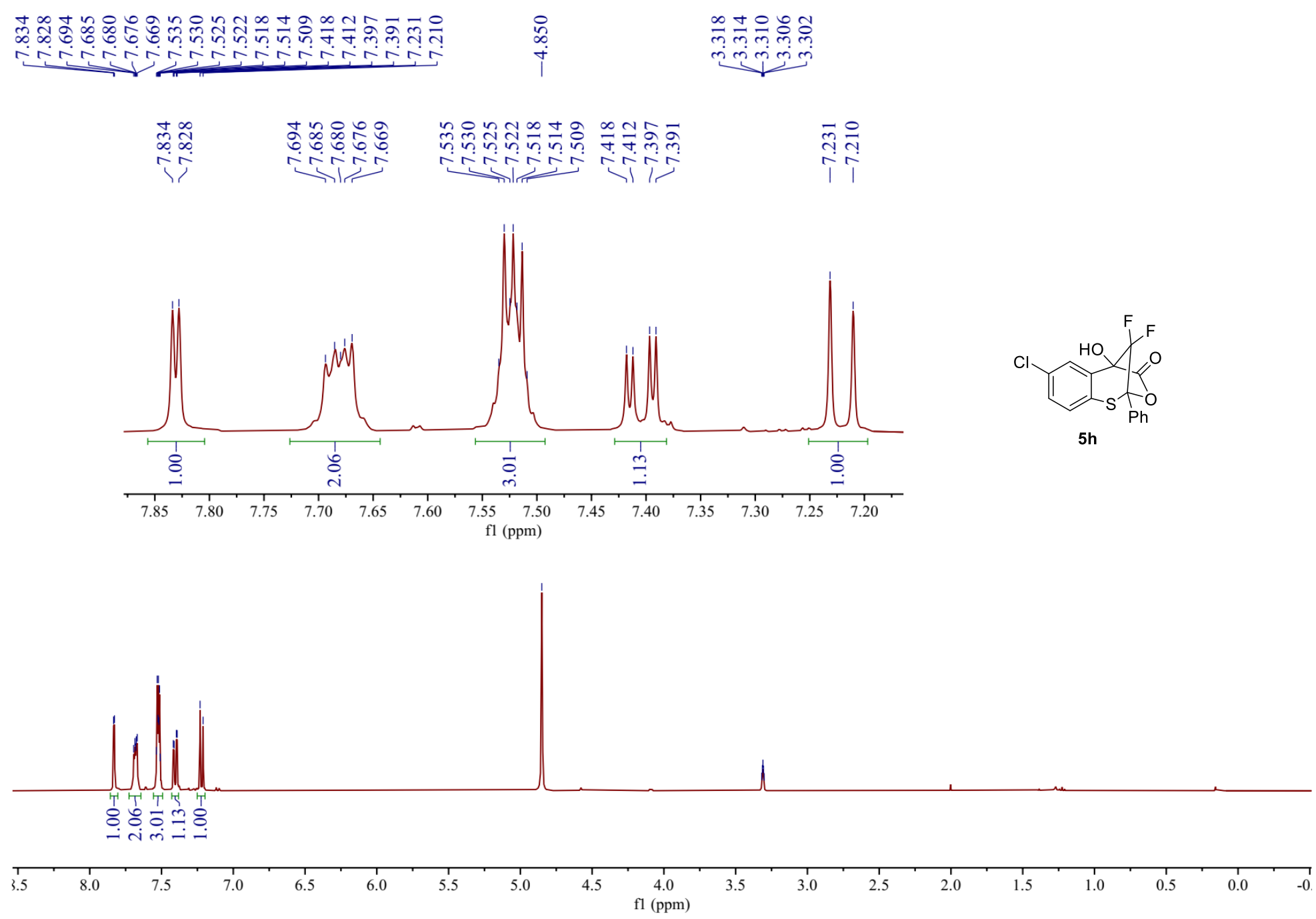

${ }^{1} \mathrm{H}$ NMR spectra of $\mathbf{5} \mathbf{h}$ in $\mathrm{CD}_{3} \mathrm{OD}(400 \mathrm{MHz})$ 

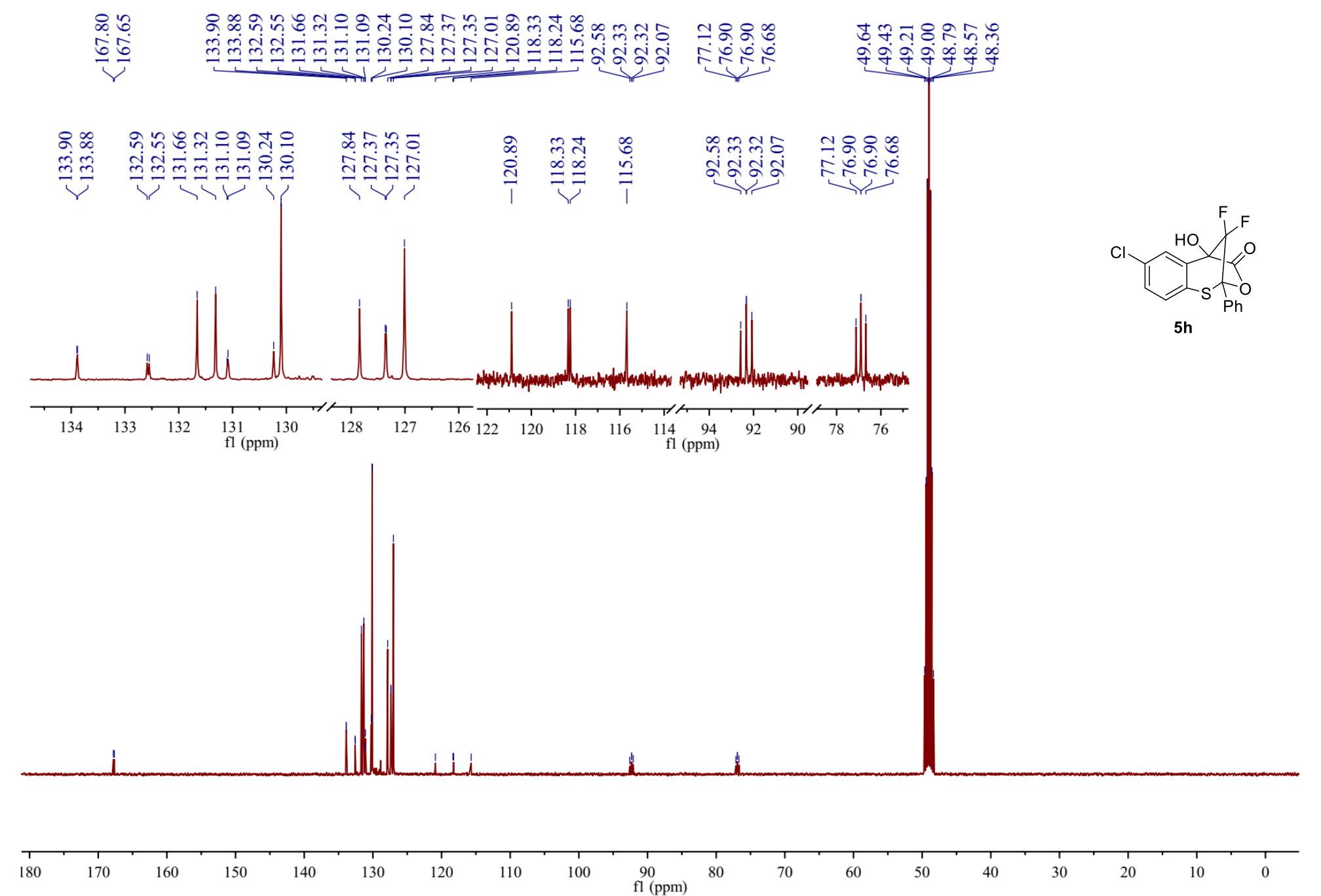

${ }^{13} \mathrm{C}\left\{{ }^{1} \mathrm{H}\right\}$ NMR spectra of $\mathbf{5 h}$ in $\mathrm{CD}_{3} \mathrm{OD}(100 \mathrm{MHz})$ 


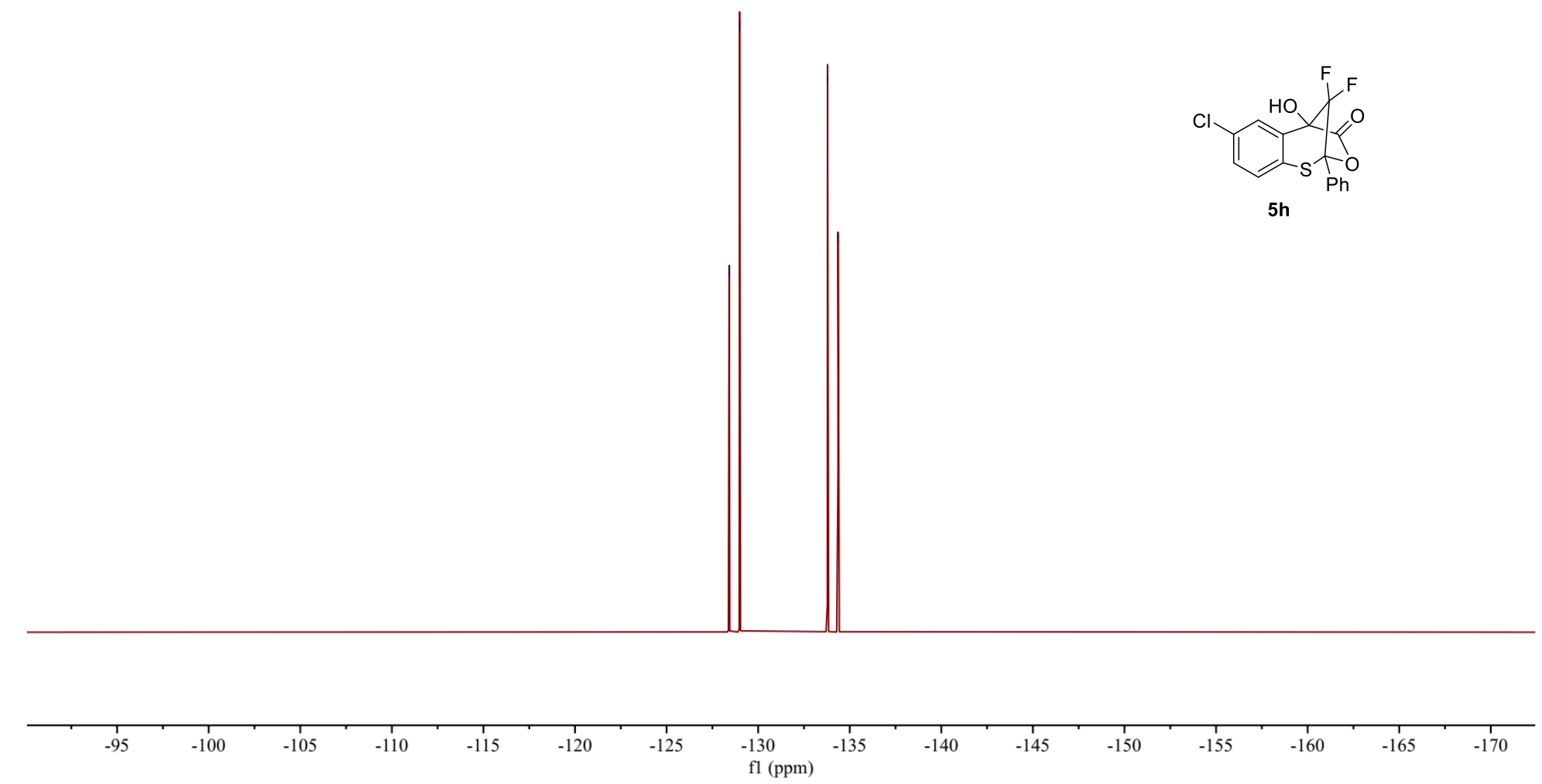

${ }^{19} \mathrm{~F}$ NMR spectra of $\mathbf{5} \mathbf{h}$ in $\mathrm{CD}_{3} \mathrm{OD}(376 \mathrm{MHz})$ 

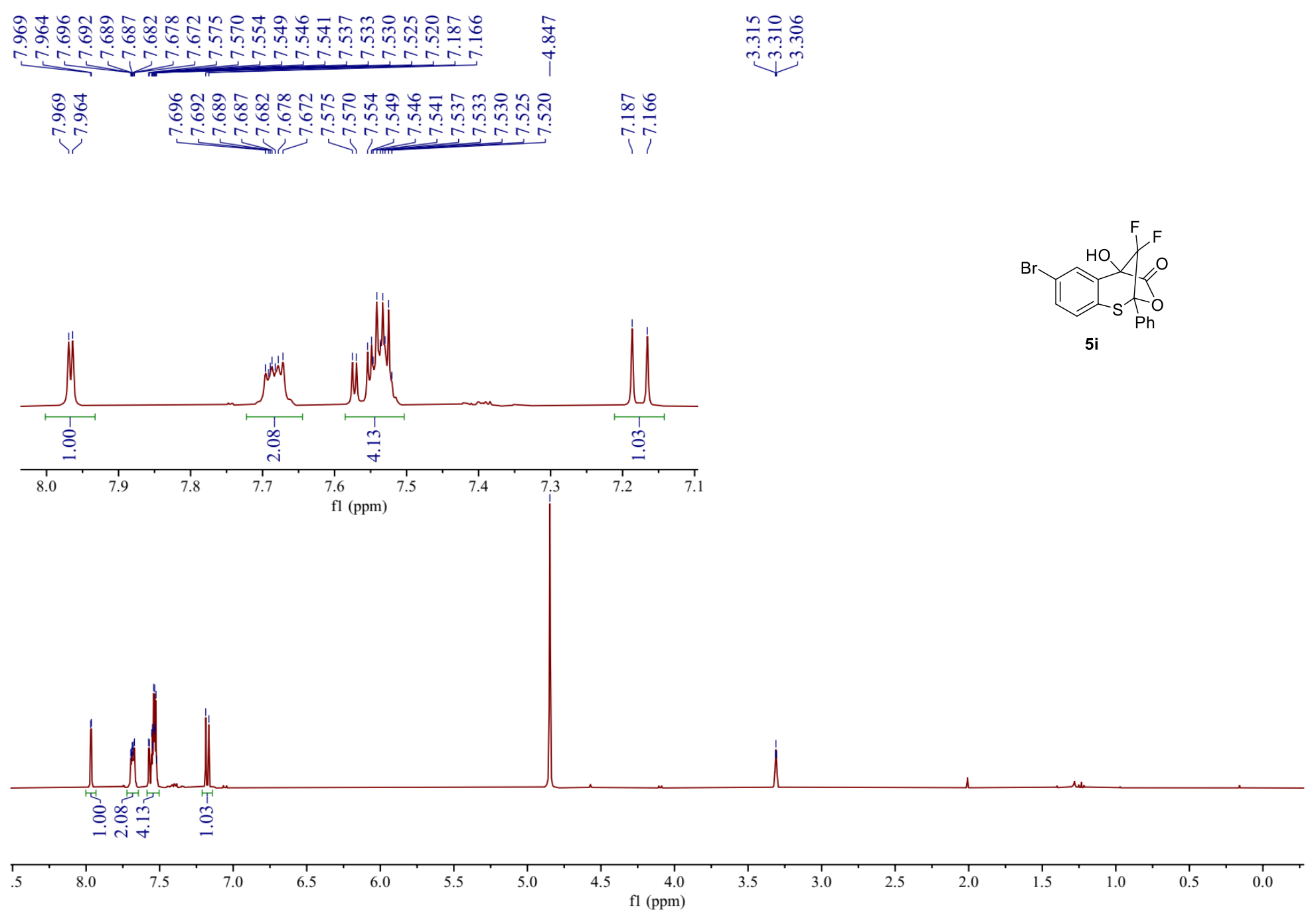

${ }^{1} \mathrm{H}$ NMR spectra of $\mathbf{5 i}$ in $\mathrm{CD}_{3} \mathrm{OD}(400 \mathrm{MHz})$ 

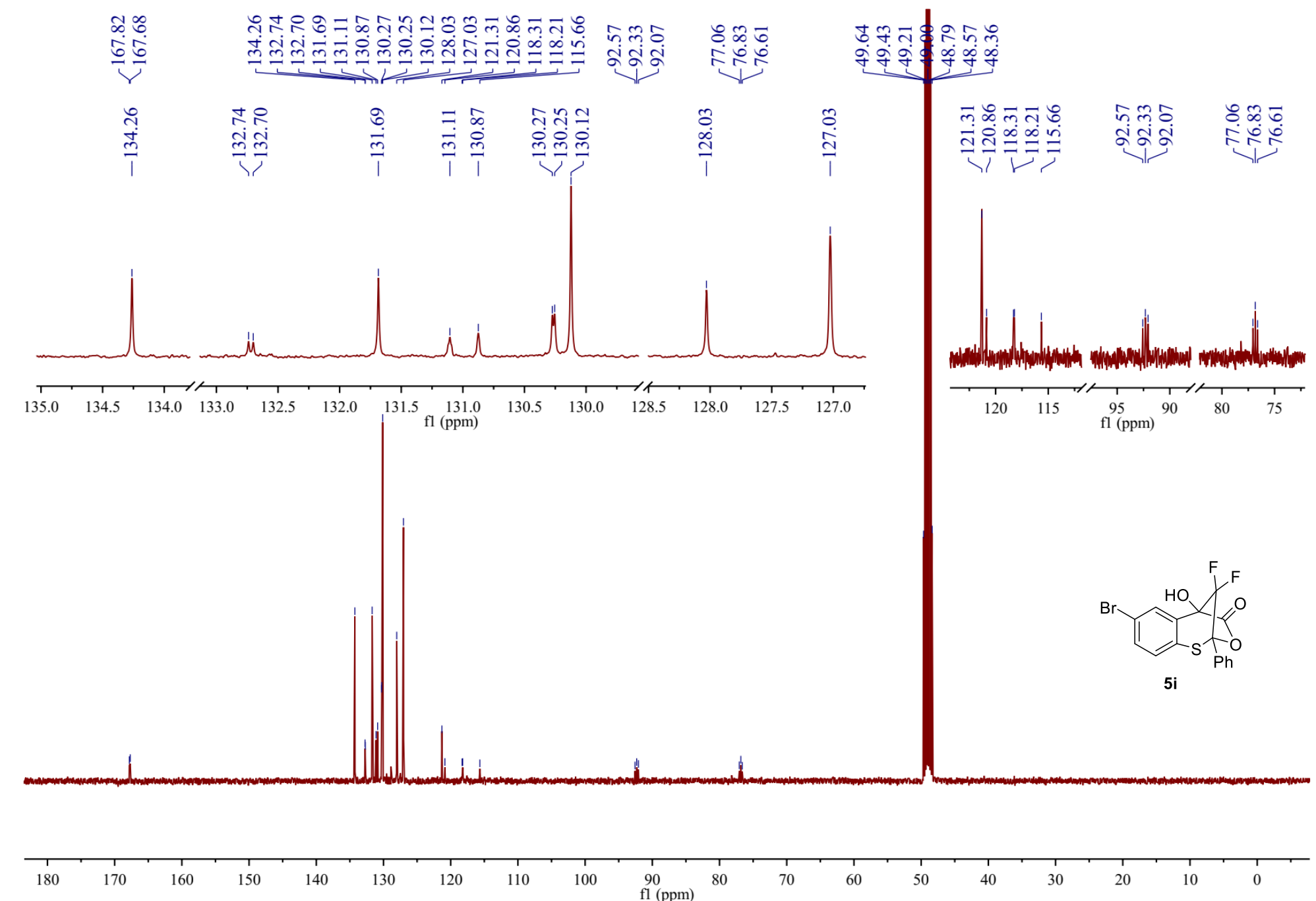

${ }^{13} \mathrm{C}\left\{{ }^{1} \mathrm{H}\right\}$ NMR spectra of $\mathbf{5 i}$ in $\mathrm{CD}_{3} \mathrm{OD}(100 \mathrm{MHz})$ 


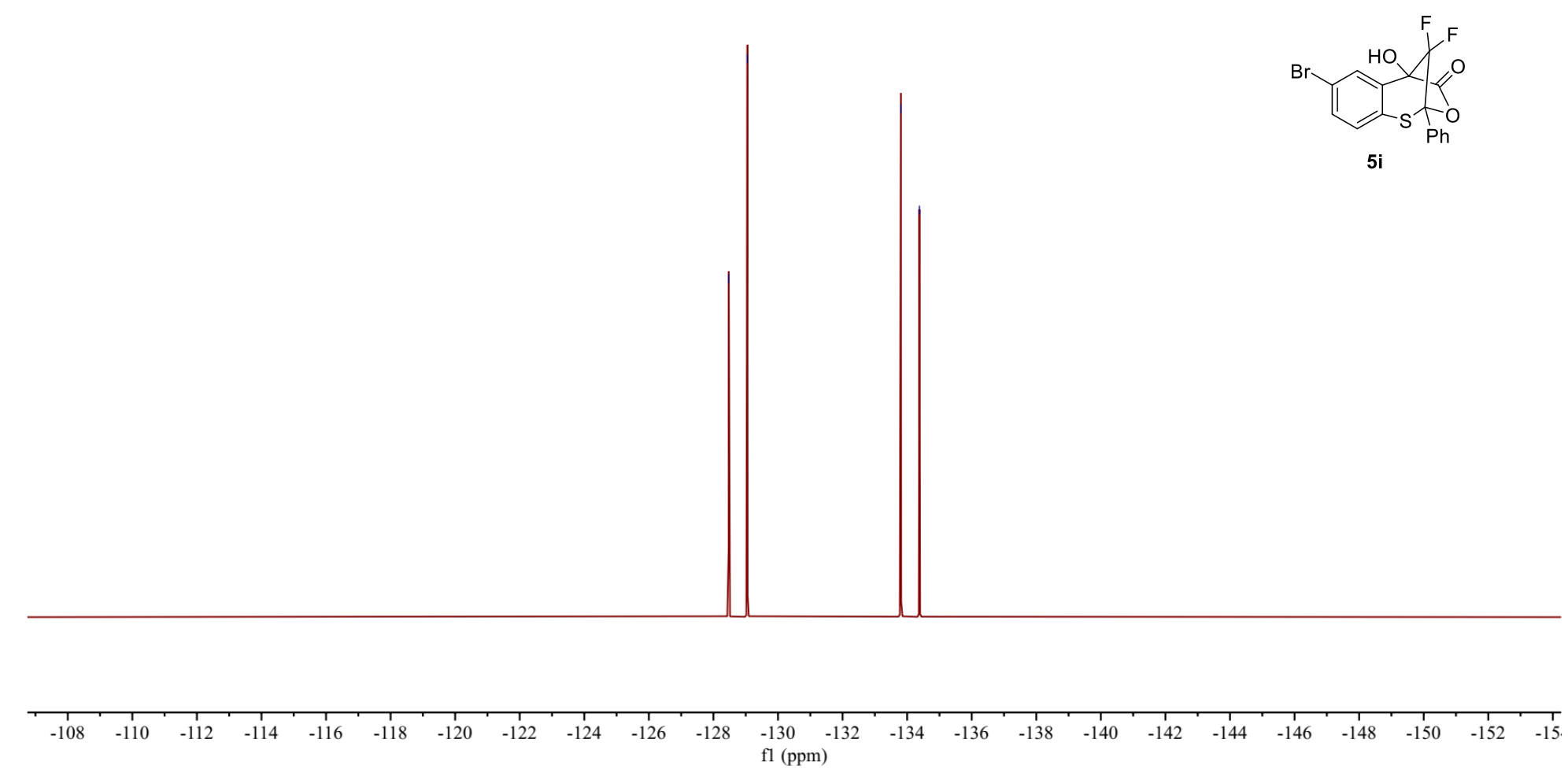

${ }^{19} \mathrm{~F}$ NMR spectra of $\mathbf{5 i}$ in $\mathrm{CD}_{3} \mathrm{OD}(376 \mathrm{MHz})$ 


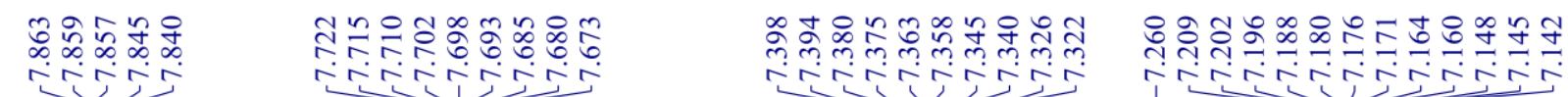
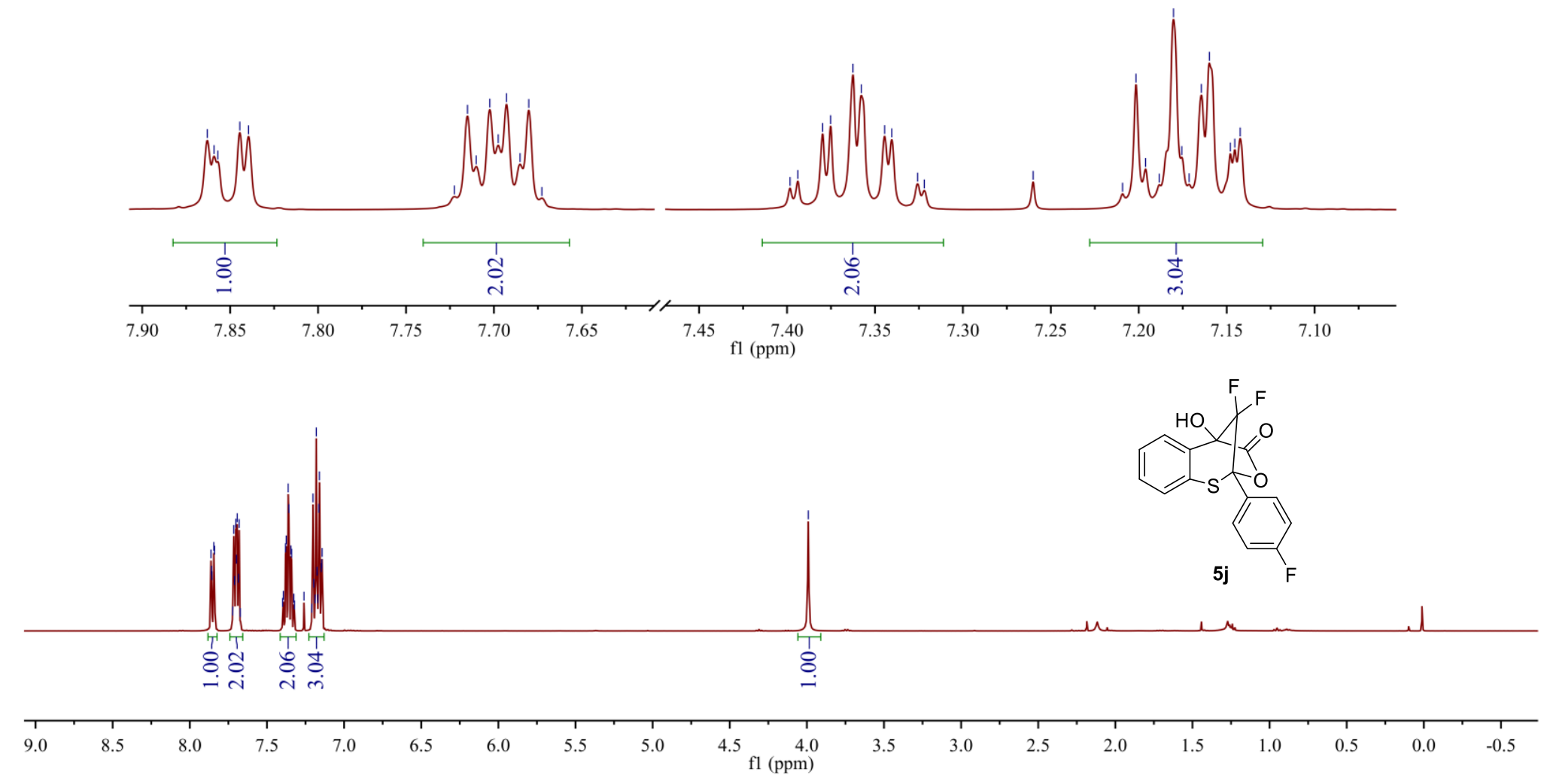

${ }^{1} \mathrm{H}$ NMR spectra of $\mathbf{5} \mathbf{j}$ in $\mathrm{CDCl}_{3}(400 \mathrm{MHz})$ 


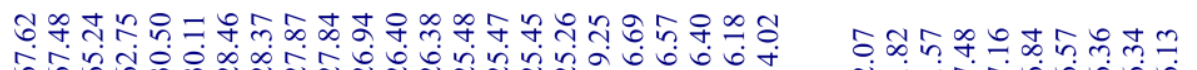

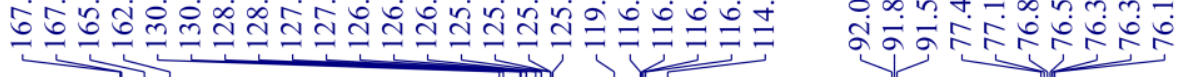

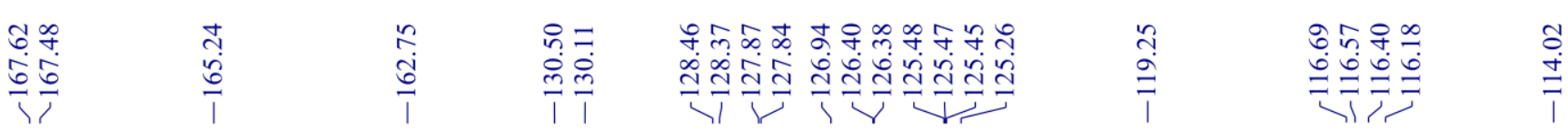

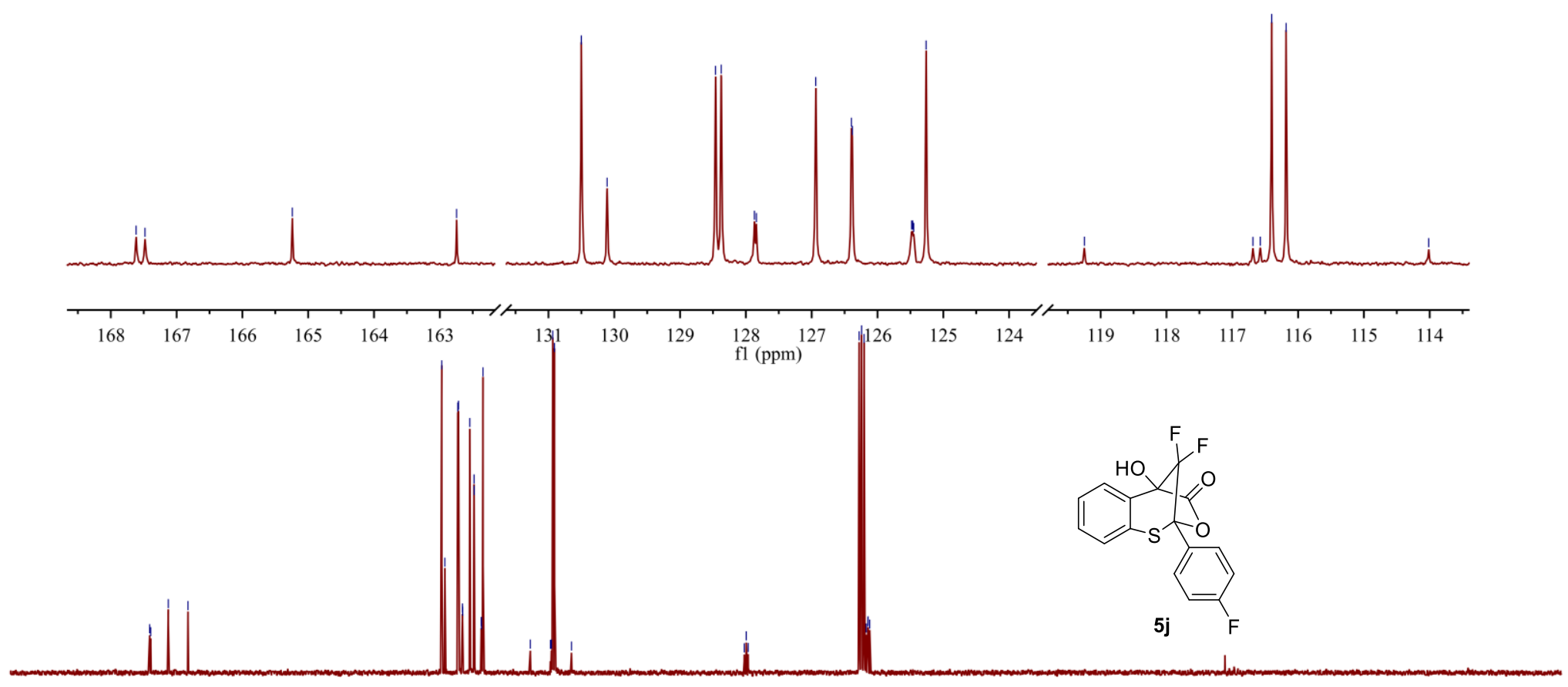

$\begin{array}{lllll}180 & 170 & 160 & 150 & 140\end{array}$

120

100

f1 (ppm) 80

${ }^{13} \mathrm{C}\left\{{ }^{1} \mathrm{H}\right\}$ NMR spectra of $\mathbf{5 j}$ in $\mathrm{CDCl}_{3}(100 \mathrm{MHz})$ 

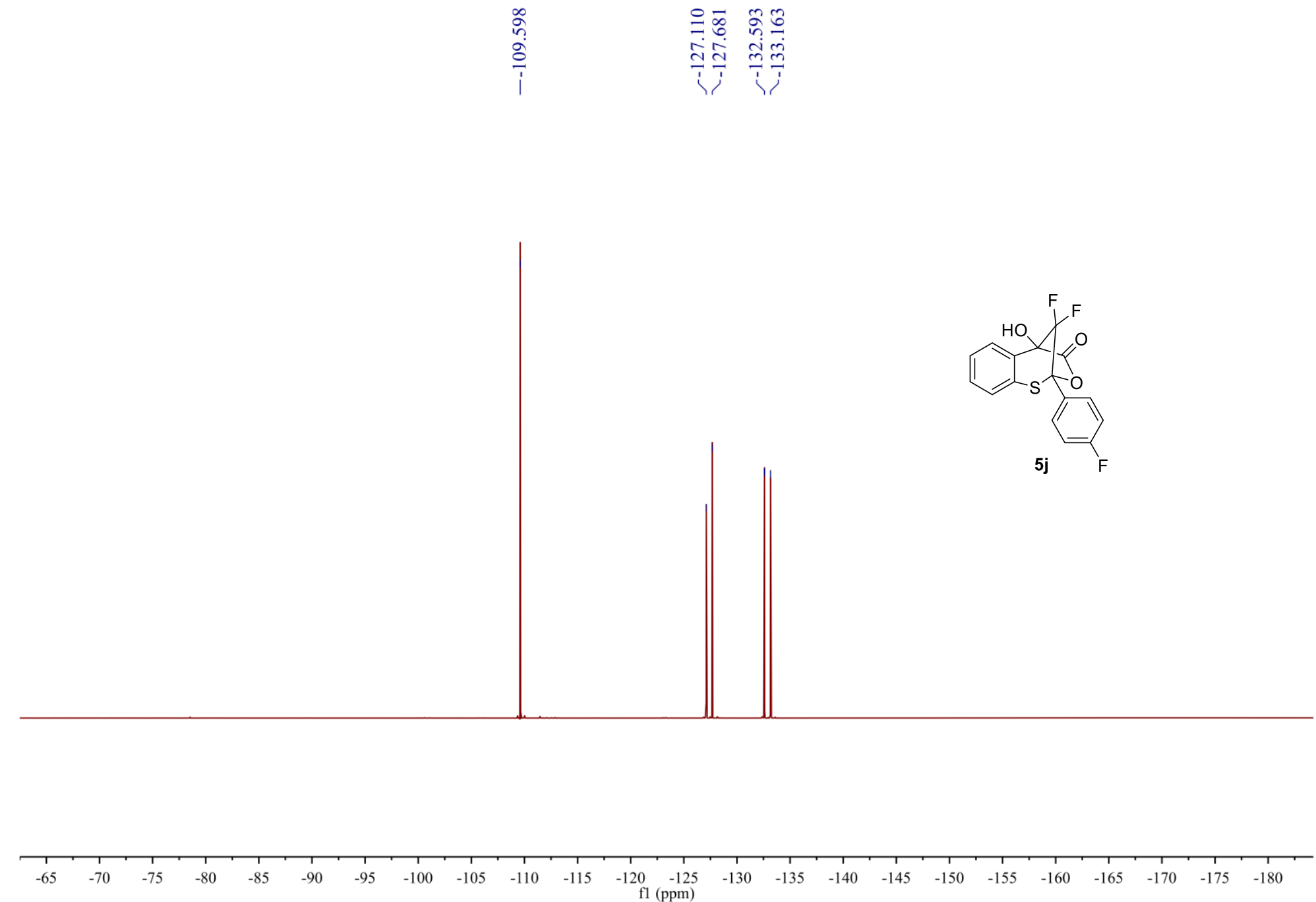

${ }^{19} \mathrm{~F}$ NMR spectra of $\mathbf{5 j}$ in $\mathrm{CDCl}_{3}(376 \mathrm{MHz})$ 


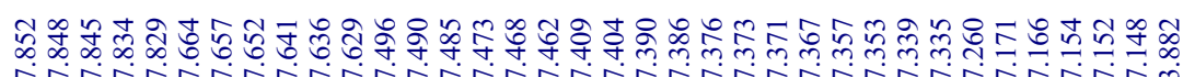

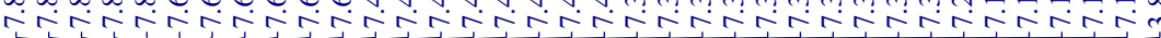
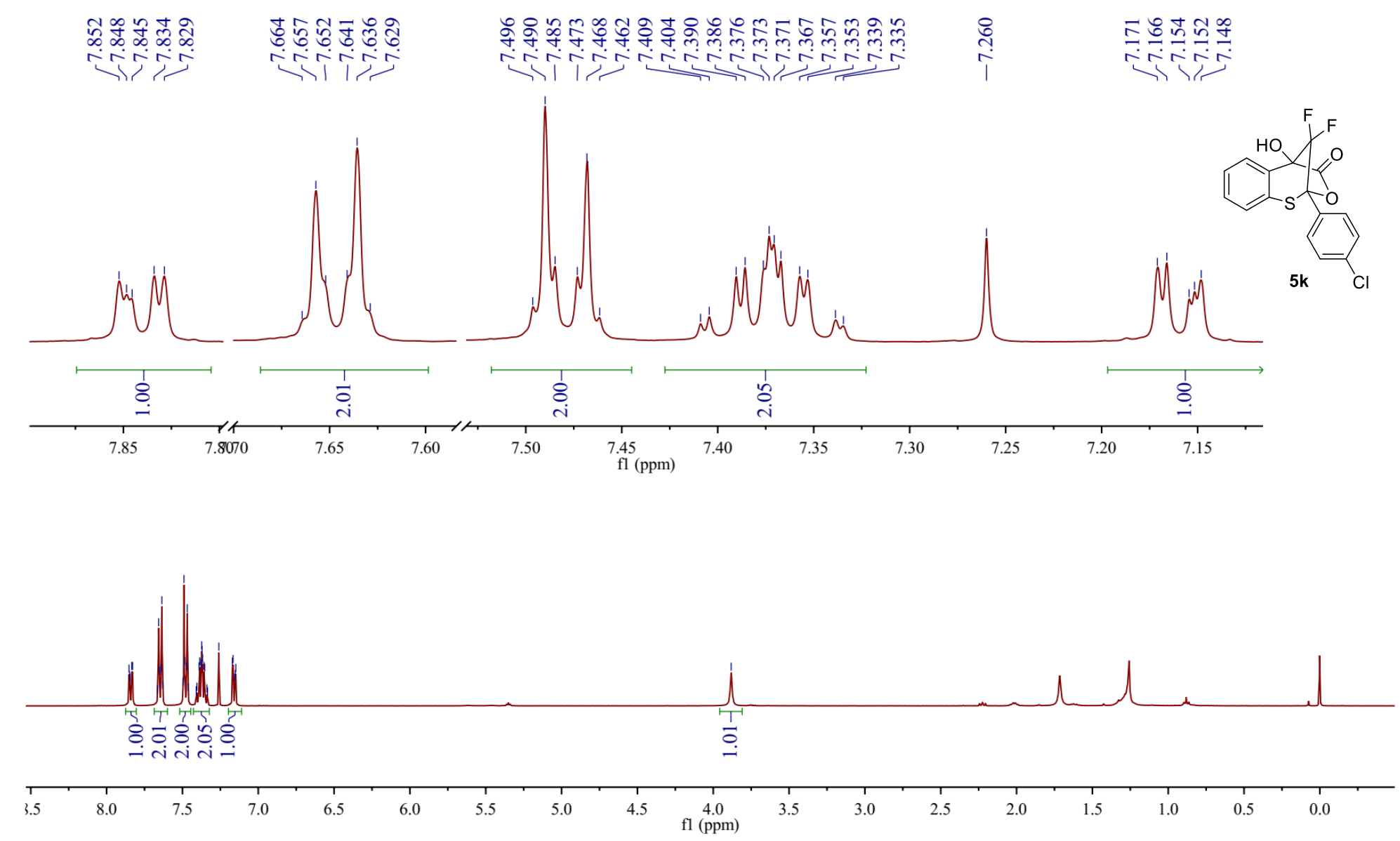

${ }^{1} \mathrm{H}$ NMR spectra of $\mathbf{5 k}$ in $\mathrm{CDCl}_{3}(400 \mathrm{MHz})$ 

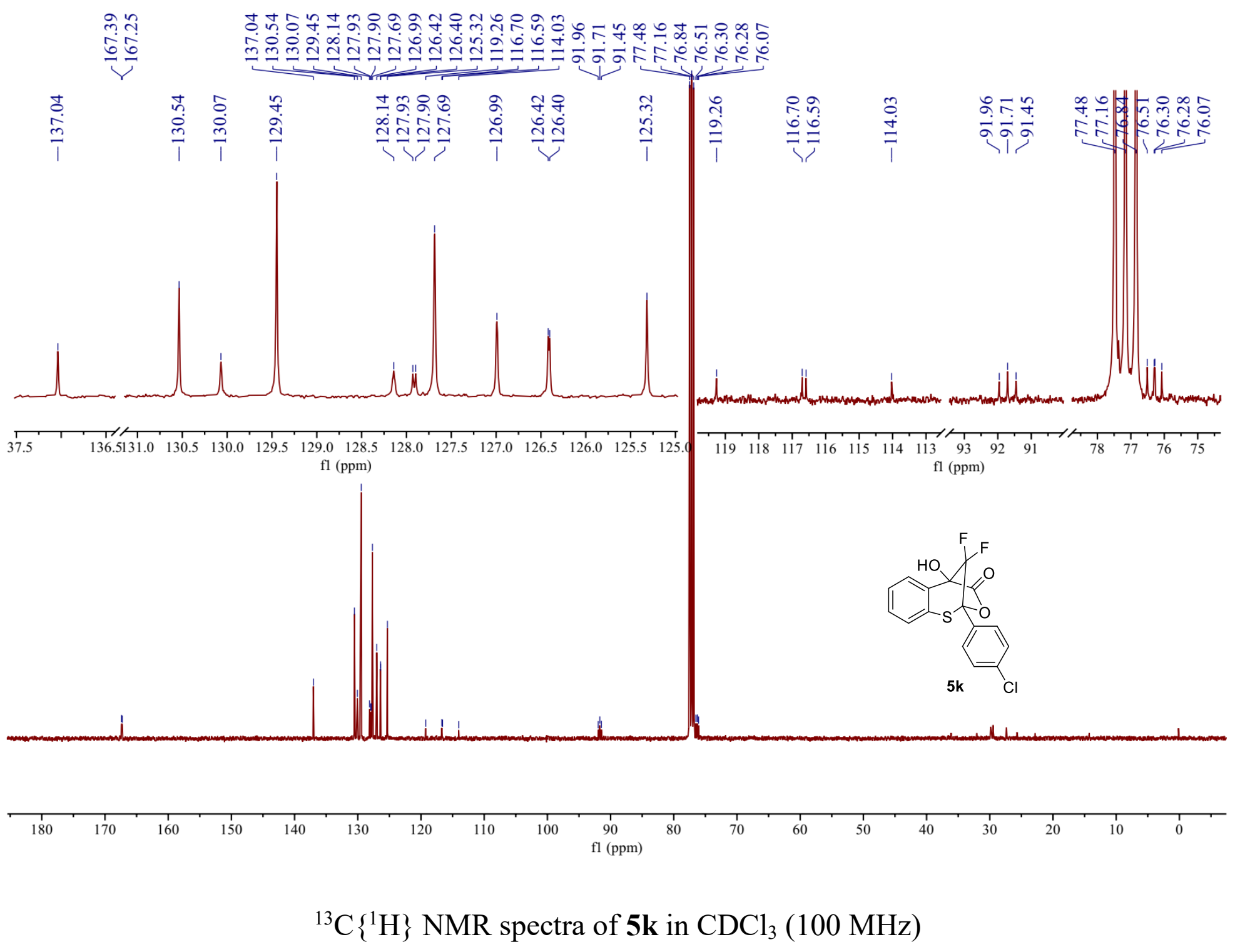


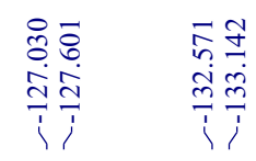

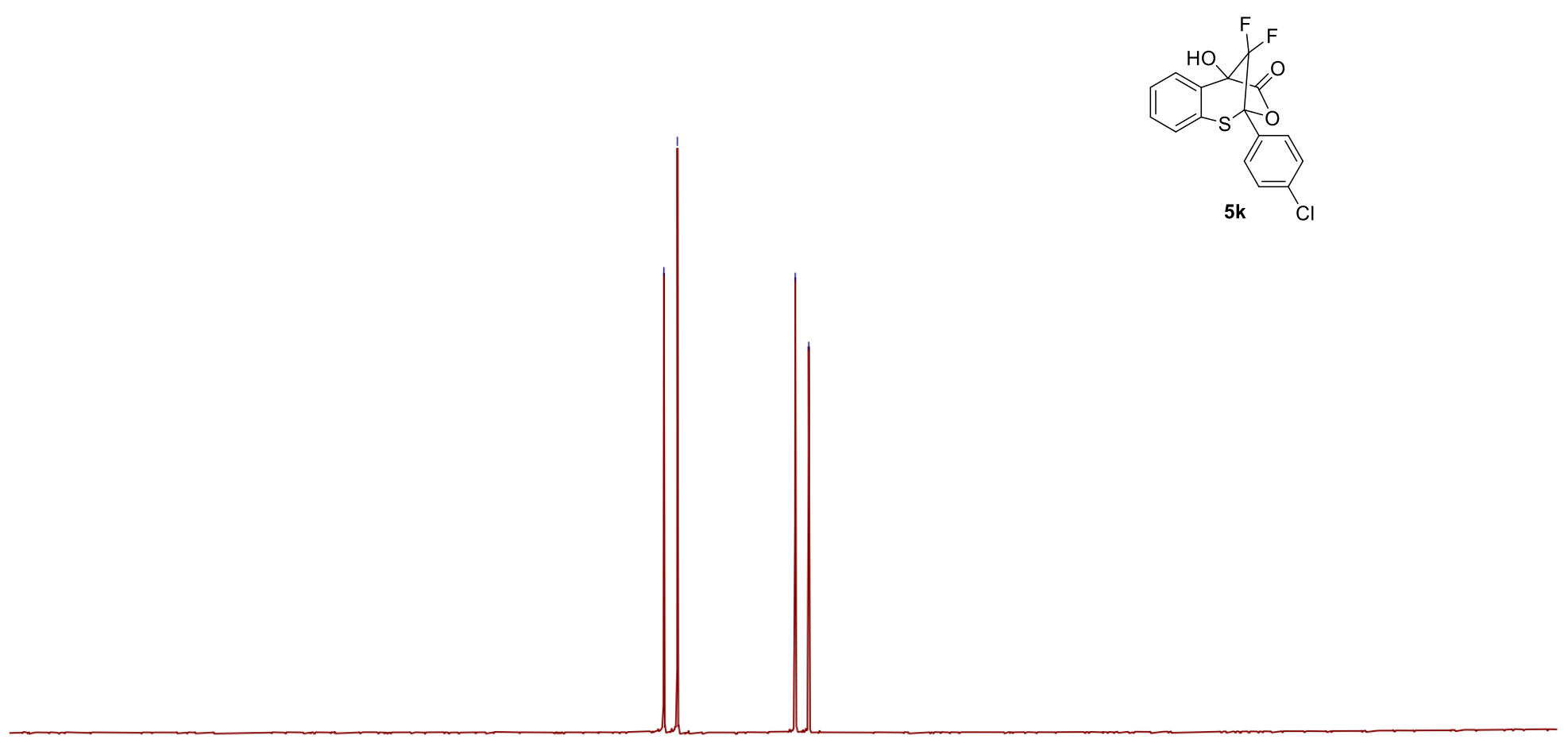

.100

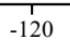

$-125$

$-130$

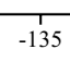

$-140$

$-145$

$-150$

$-155$

${ }^{19} \mathrm{~F}$ NMR spectra of $\mathbf{5 k}$ in $\mathrm{CDCl}_{3}(376 \mathrm{MHz})$ 


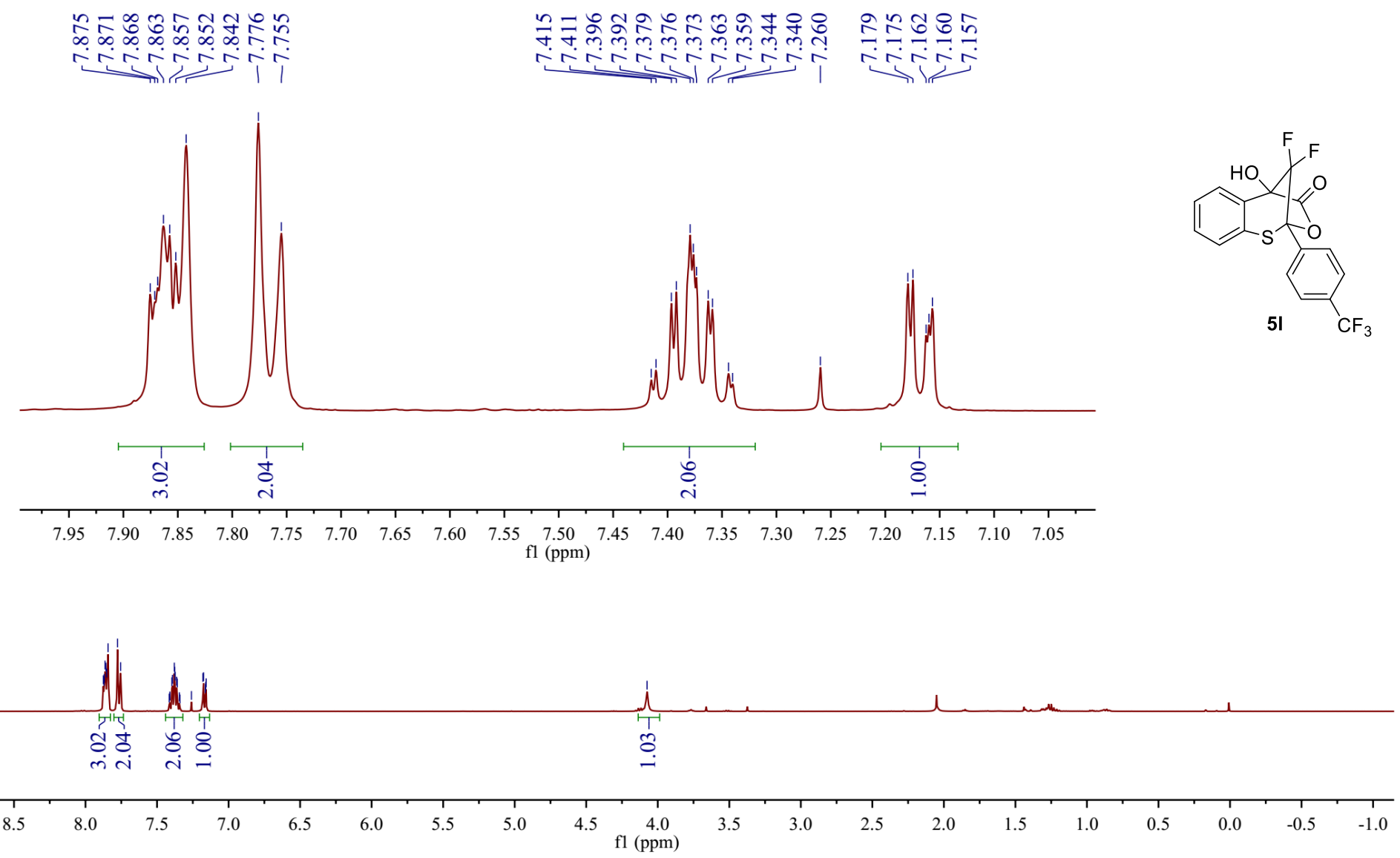

${ }^{1} \mathrm{H}$ NMR spectra of $\mathbf{5 l}$ in $\mathrm{CDCl}_{3}(400 \mathrm{MHz})$ 


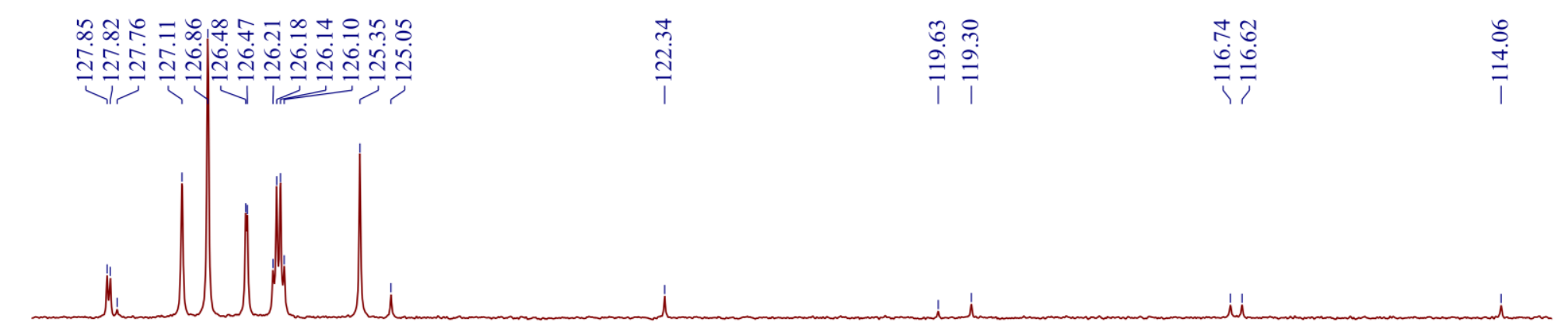

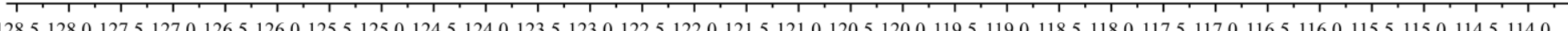
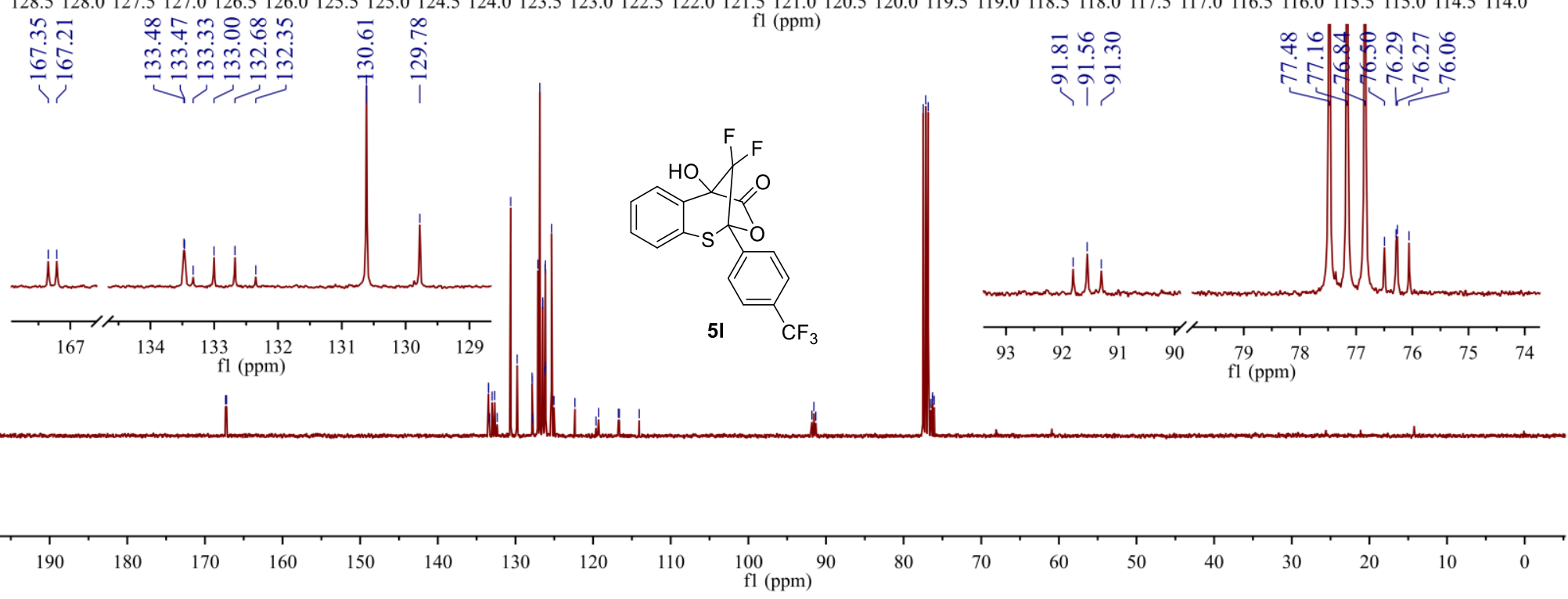

${ }^{13} \mathrm{C}\left\{{ }^{1} \mathrm{H}\right\}$ NMR spectra of $\mathbf{5 l}$ in $\mathrm{CDCl}_{3}(100 \mathrm{MHz})$ 


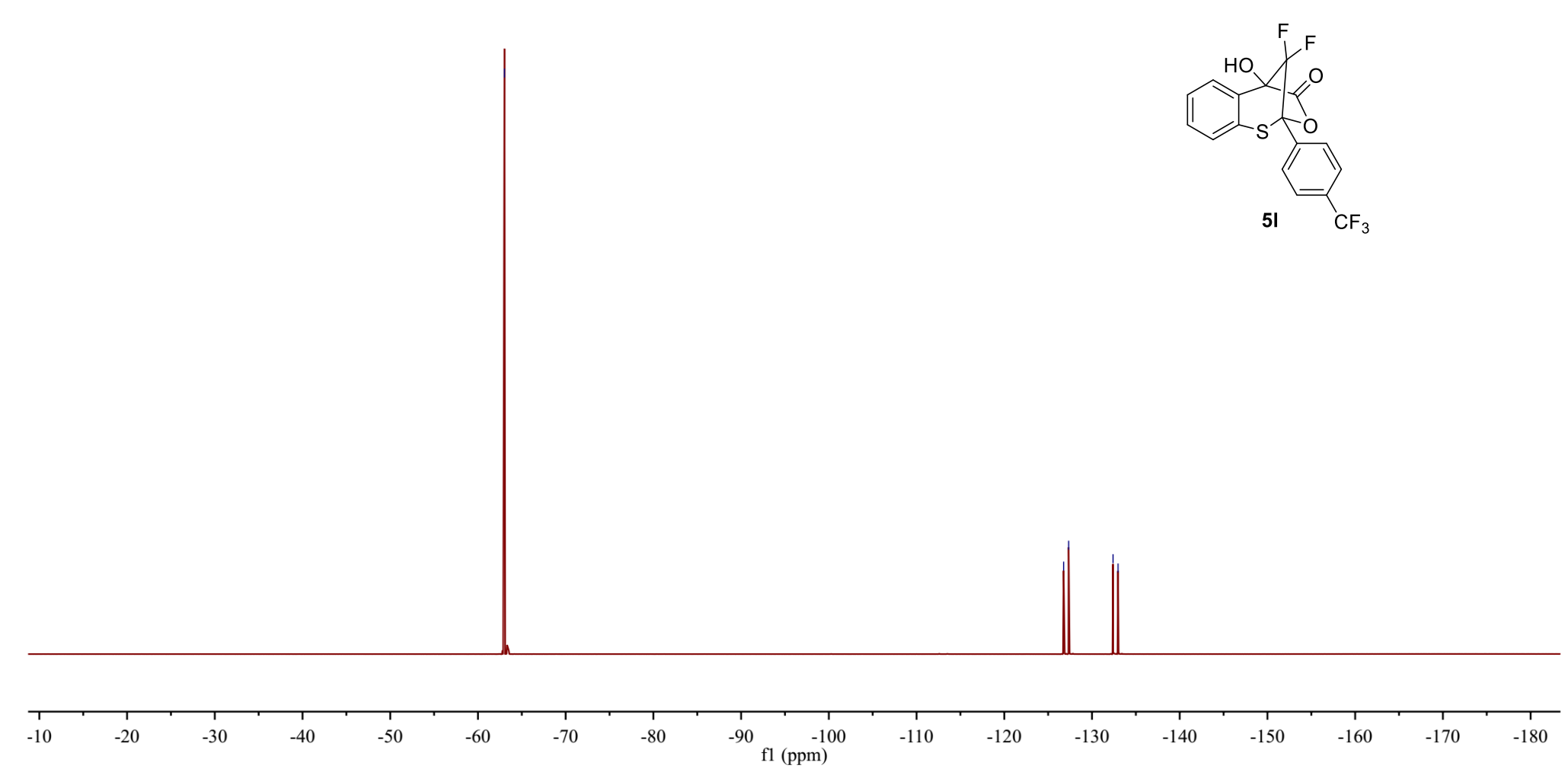

${ }^{19} \mathrm{~F}$ NMR spectra of $\mathbf{5} \mathbf{l}$ in $\mathrm{CDCl}_{3}(376 \mathrm{MHz})$ 


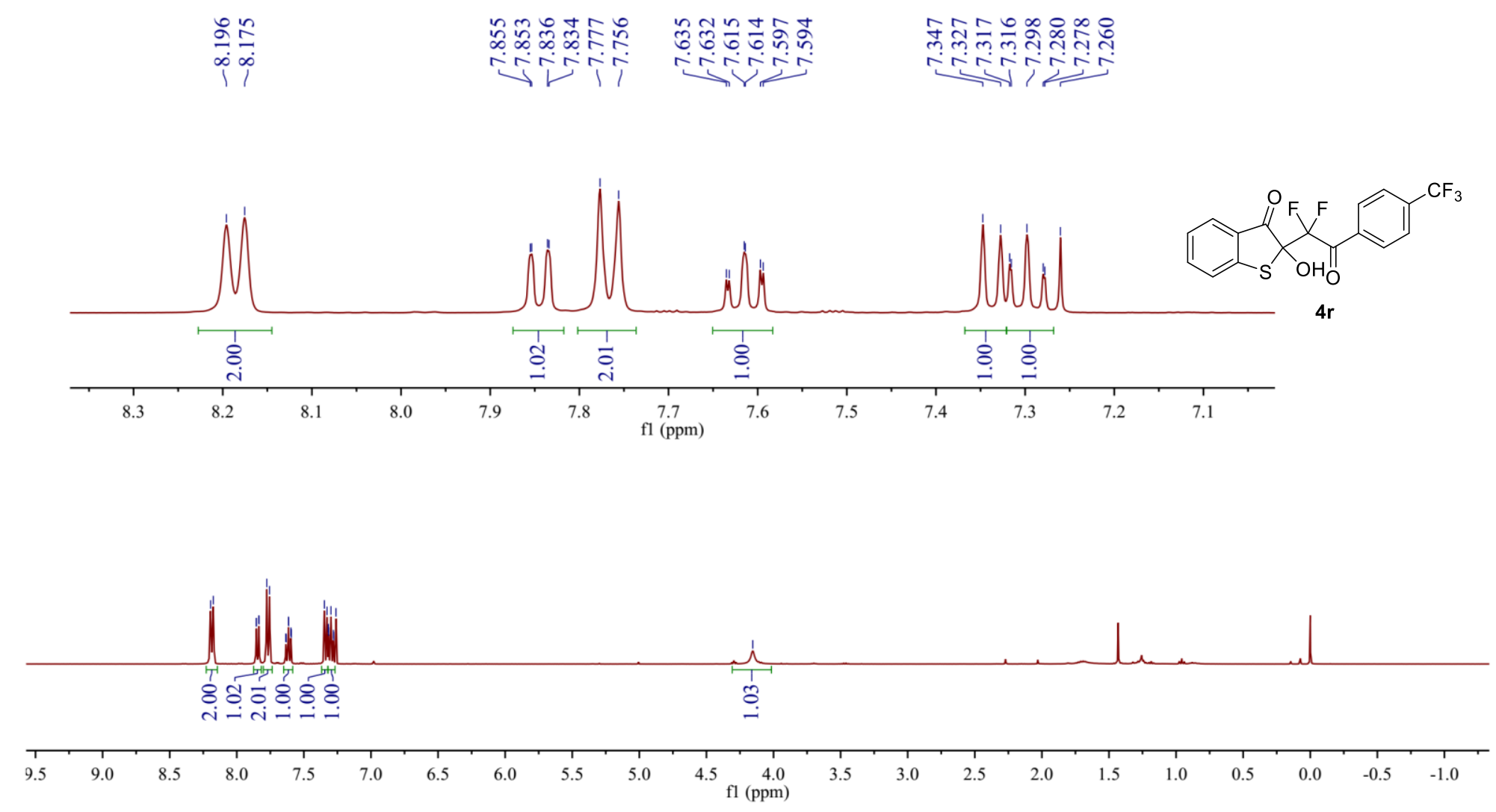

${ }^{1} \mathrm{H}$ NMR spectra of $4 \mathbf{r}$ in $\mathrm{CDCl}_{3}(400 \mathrm{MHz})$ 


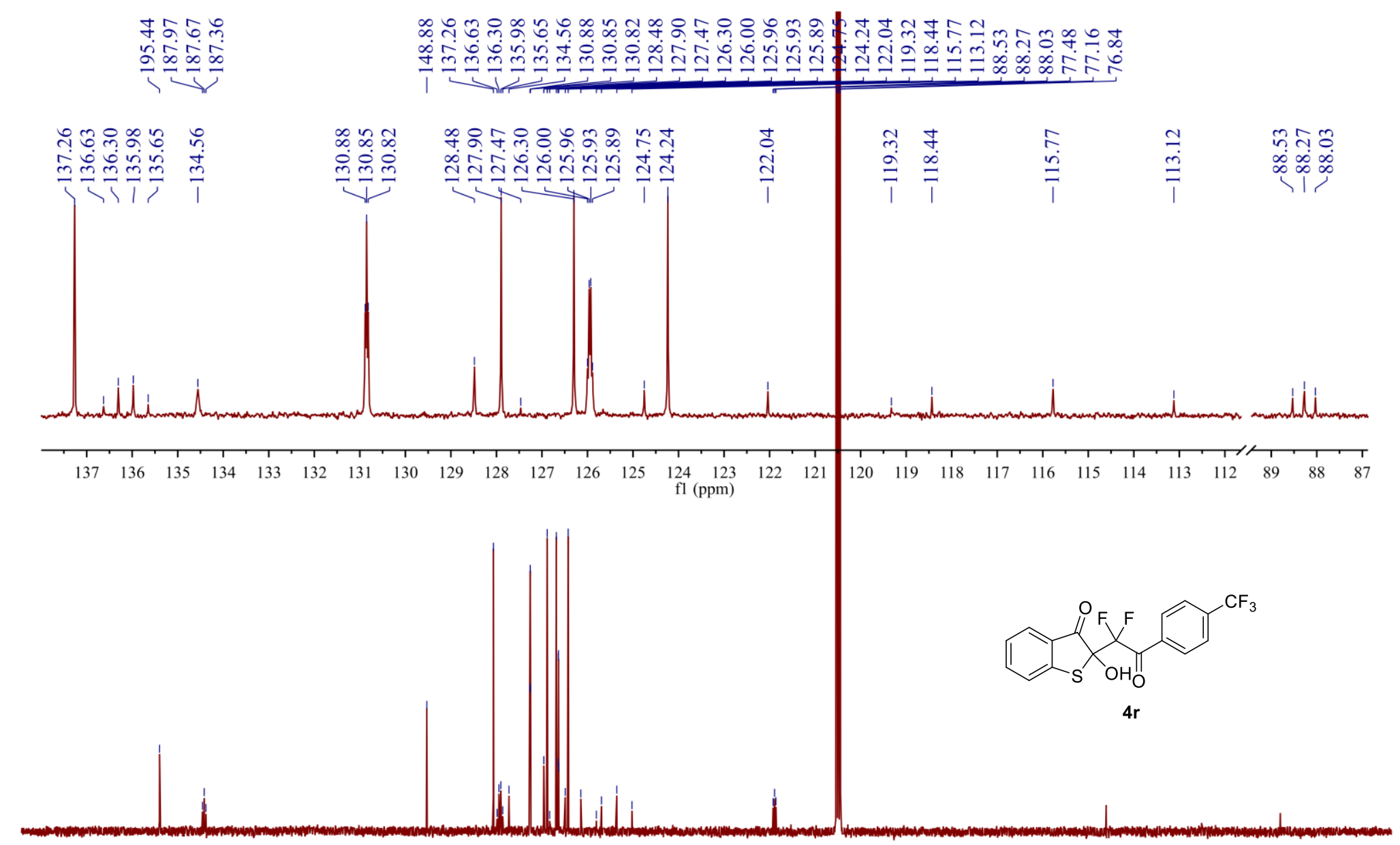

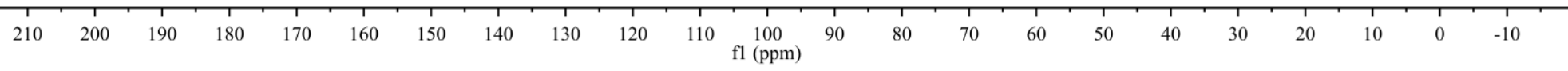

${ }^{13} \mathrm{C}\left\{{ }^{1} \mathrm{H}\right\}$ NMR spectra of $\mathbf{4 r}$ in $\mathrm{CDCl}_{3}(100 \mathrm{MHz})$ 


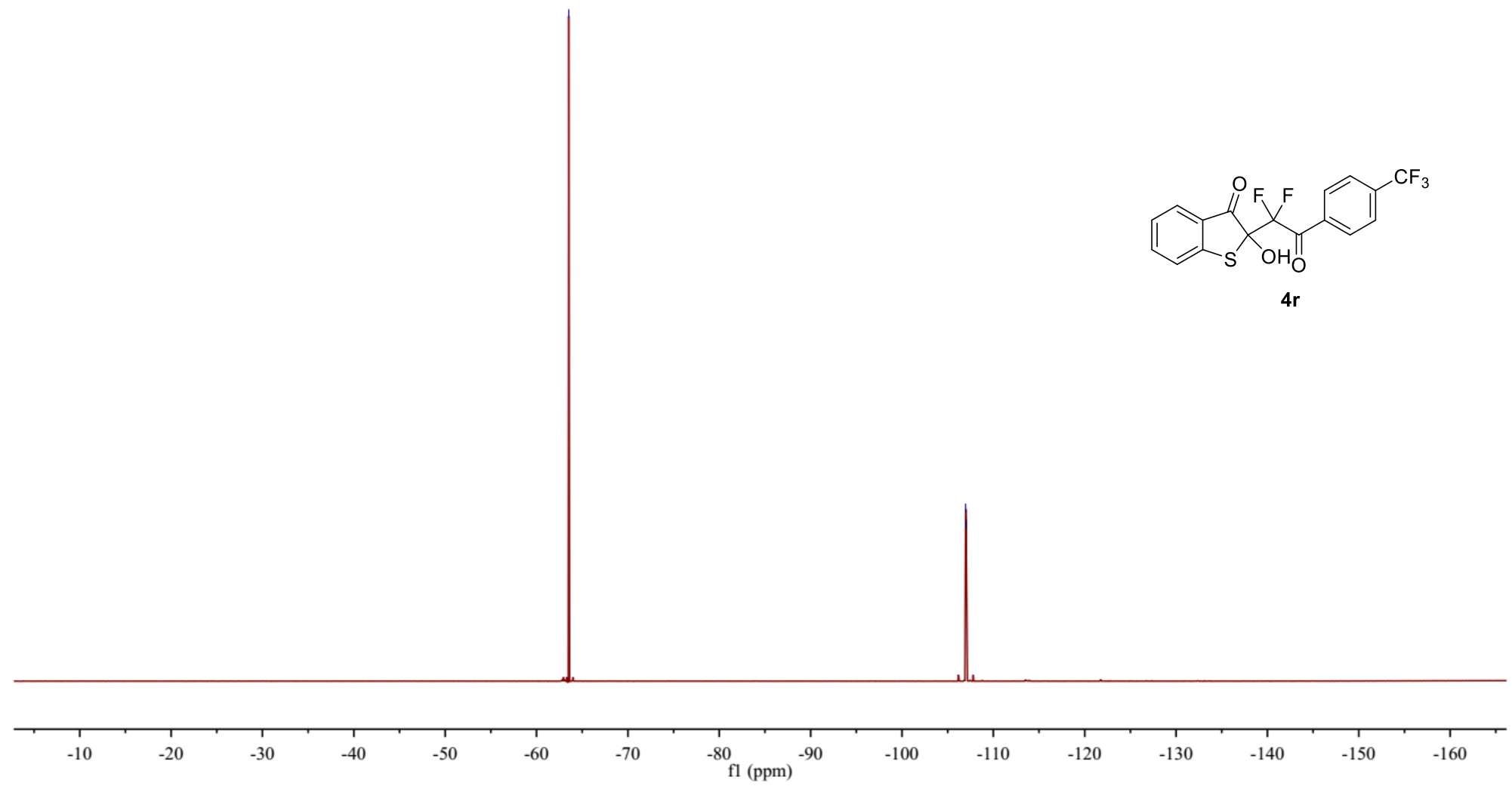

${ }^{19} \mathrm{~F}$ NMR spectra of $\mathbf{4 r}$ in $\mathrm{CDCl}_{3}(376 \mathrm{MHz})$ 


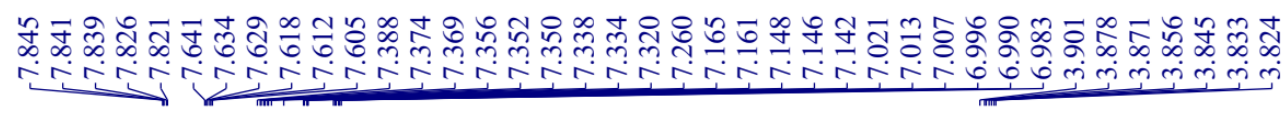

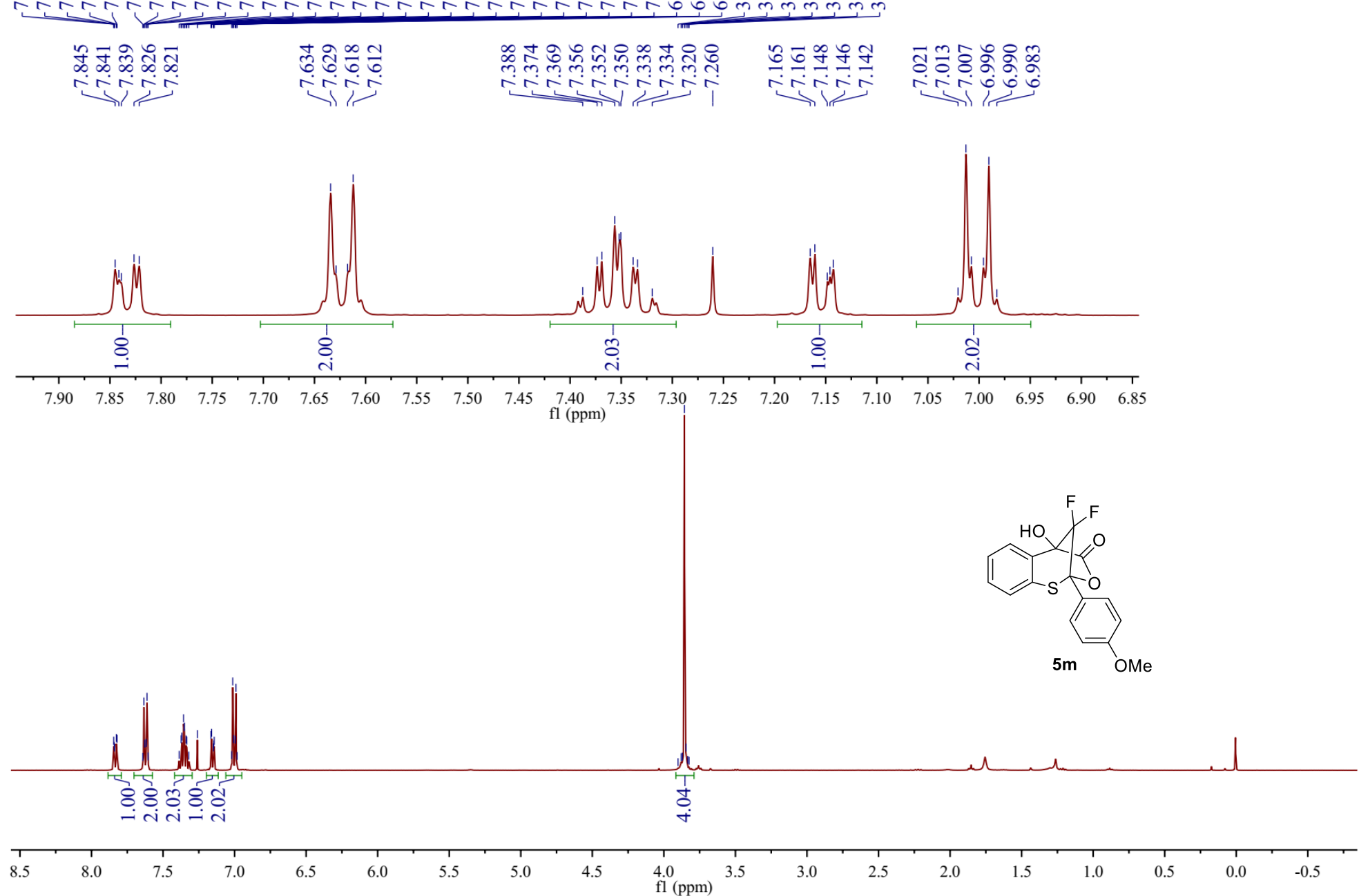

${ }^{1} \mathrm{H}$ NMR spectra of $\mathbf{5 m}$ in $\mathrm{CDCl}_{3}(400 \mathrm{MHz})$ 

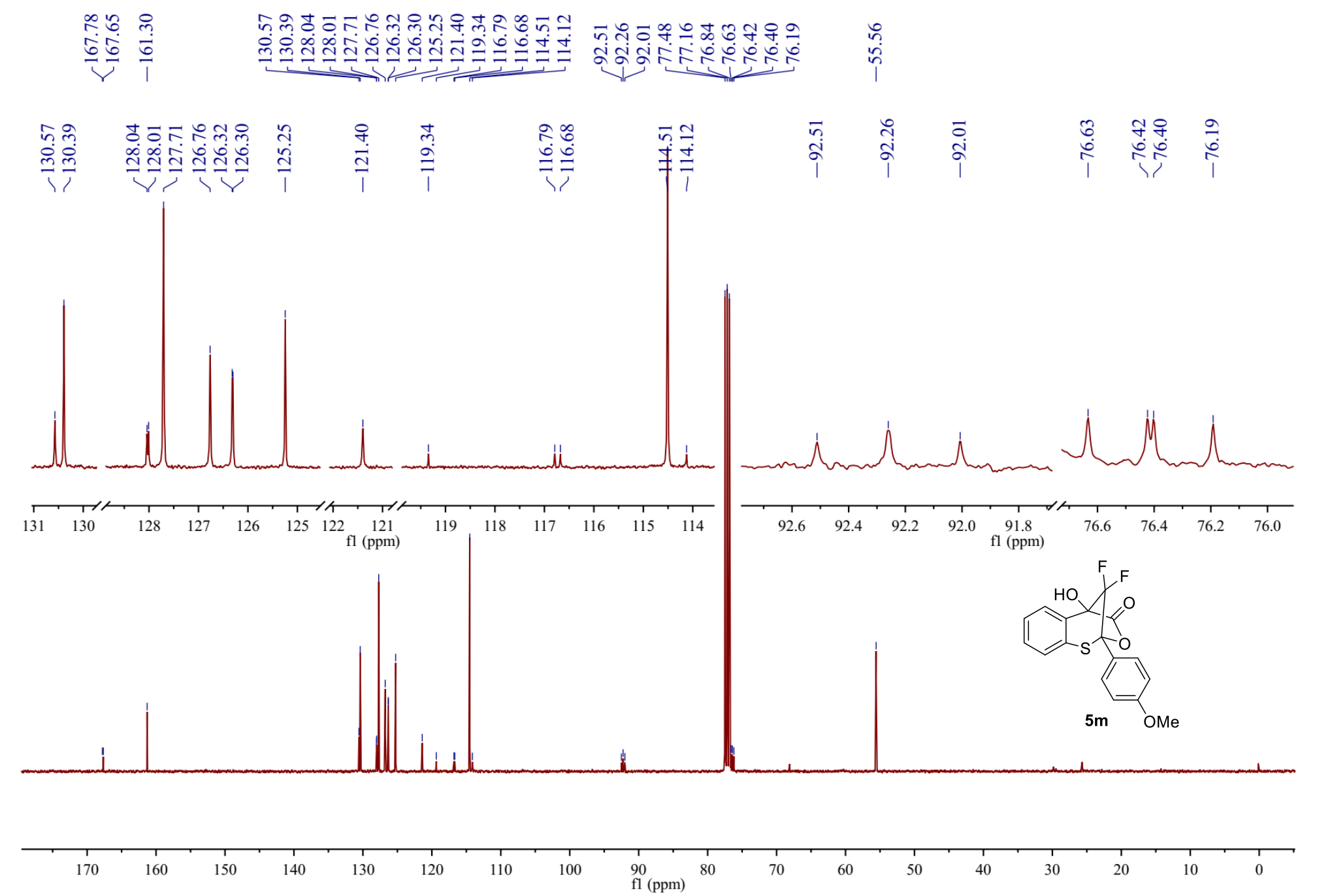

${ }^{13} \mathrm{C}\left\{{ }^{1} \mathrm{H}\right\}$ NMR spectra of $\mathbf{5} \mathbf{m}$ in $\mathrm{CDCl}_{3}(100 \mathrm{MHz})$ 


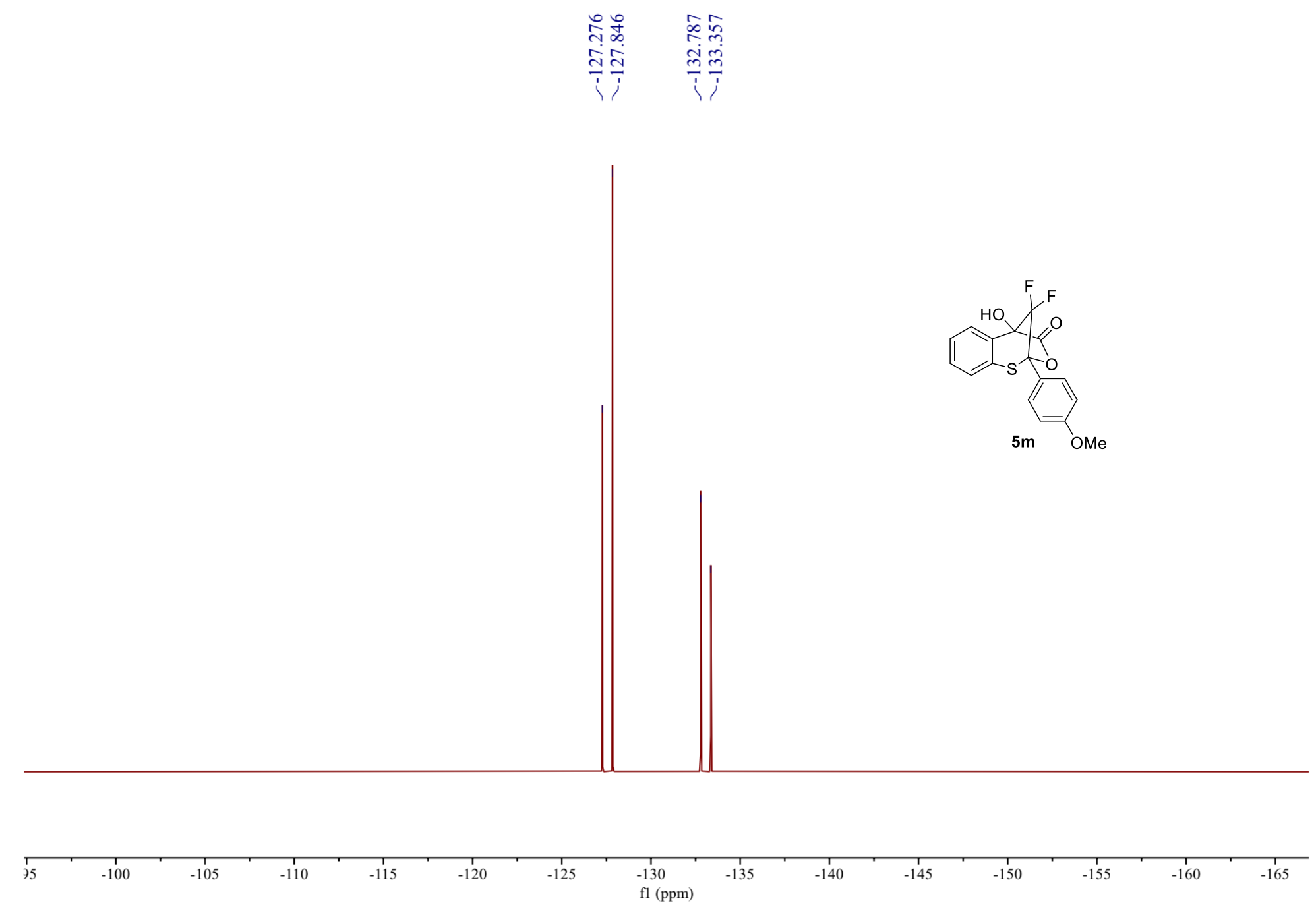

${ }^{19} \mathrm{~F}$ NMR spectra of $\mathbf{5 m}$ in $\mathrm{CDCl}_{3}(376 \mathrm{MHz})$ 

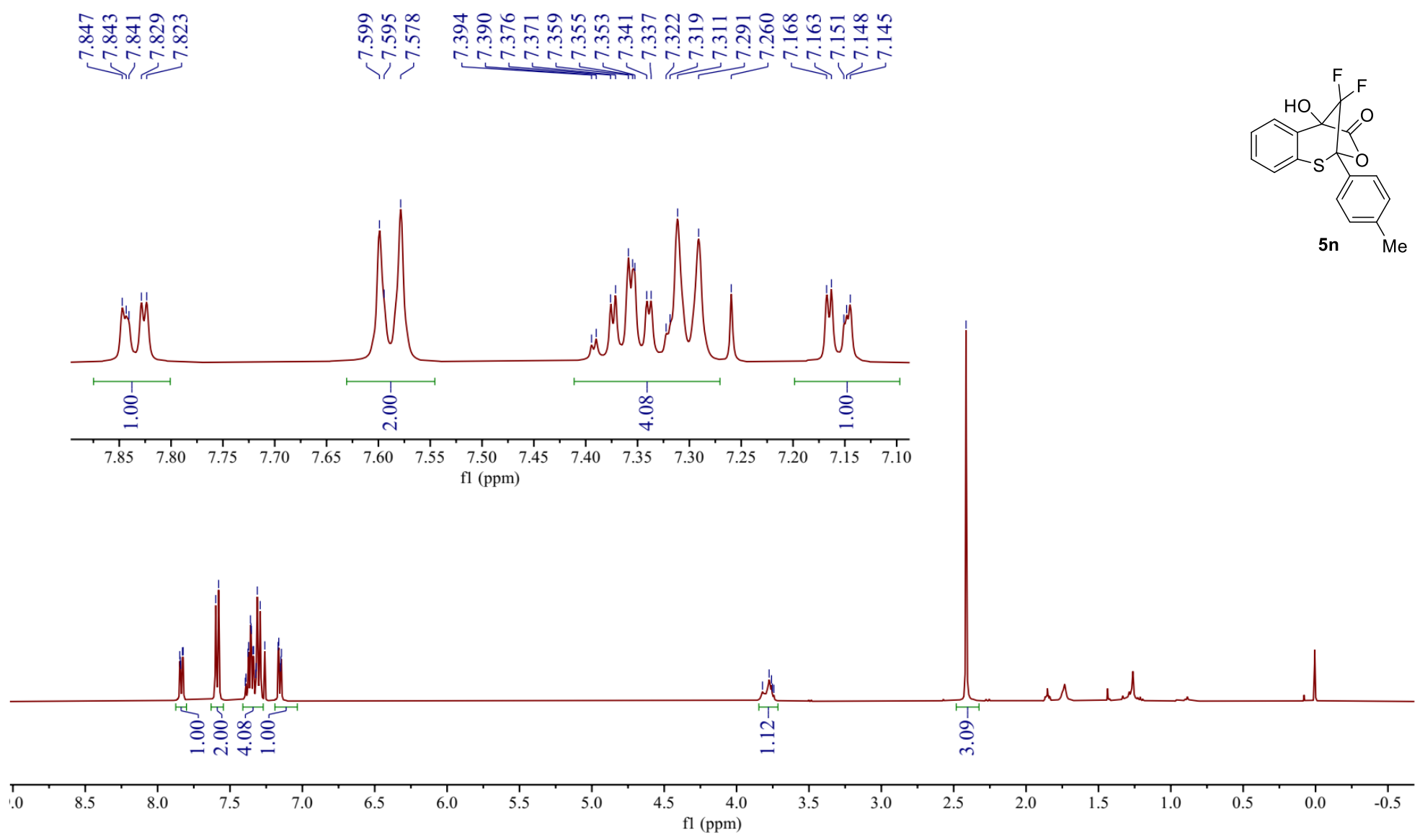

${ }^{1} \mathrm{H}$ NMR spectra of $\mathbf{5 n}$ in $\mathrm{CDCl}_{3}(400 \mathrm{MHz})$ 


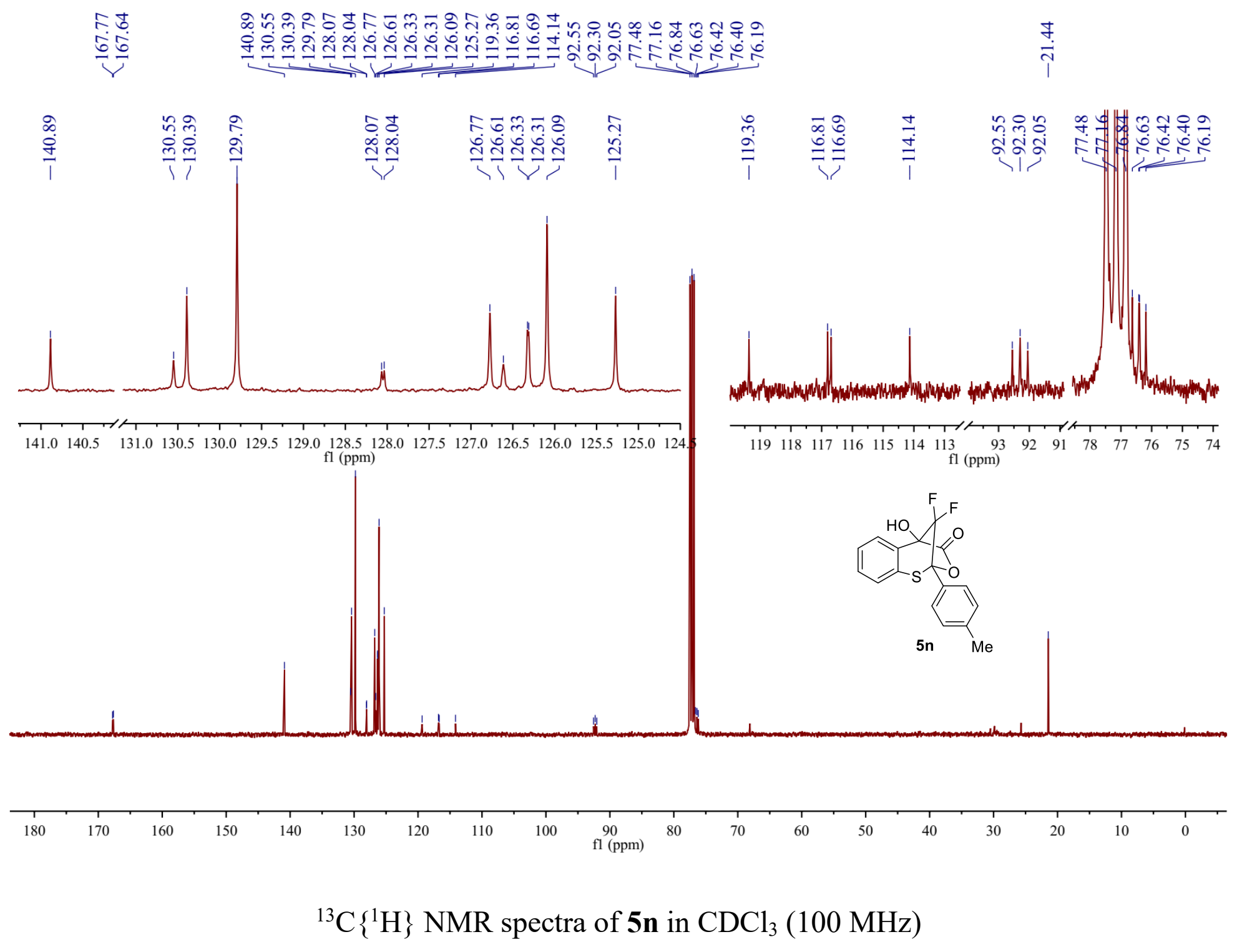




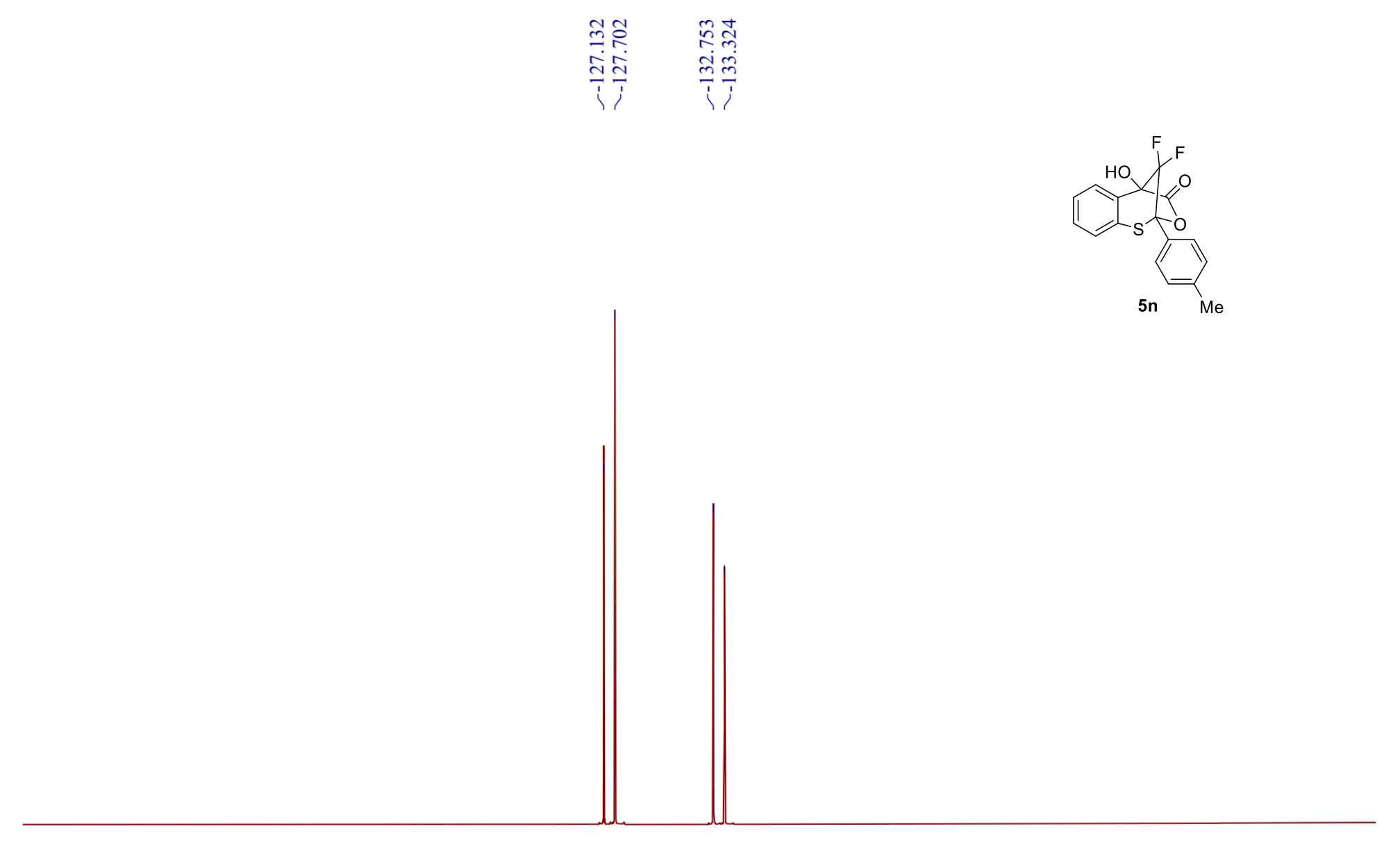

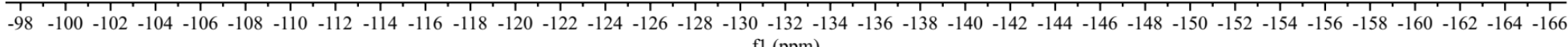
$\mathrm{fl}(\mathrm{ppm})$

${ }^{19} \mathrm{~F}$ NMR spectra of $\mathbf{5 n}$ in $\mathrm{CDCl}_{3}(376 \mathrm{MHz})$ 


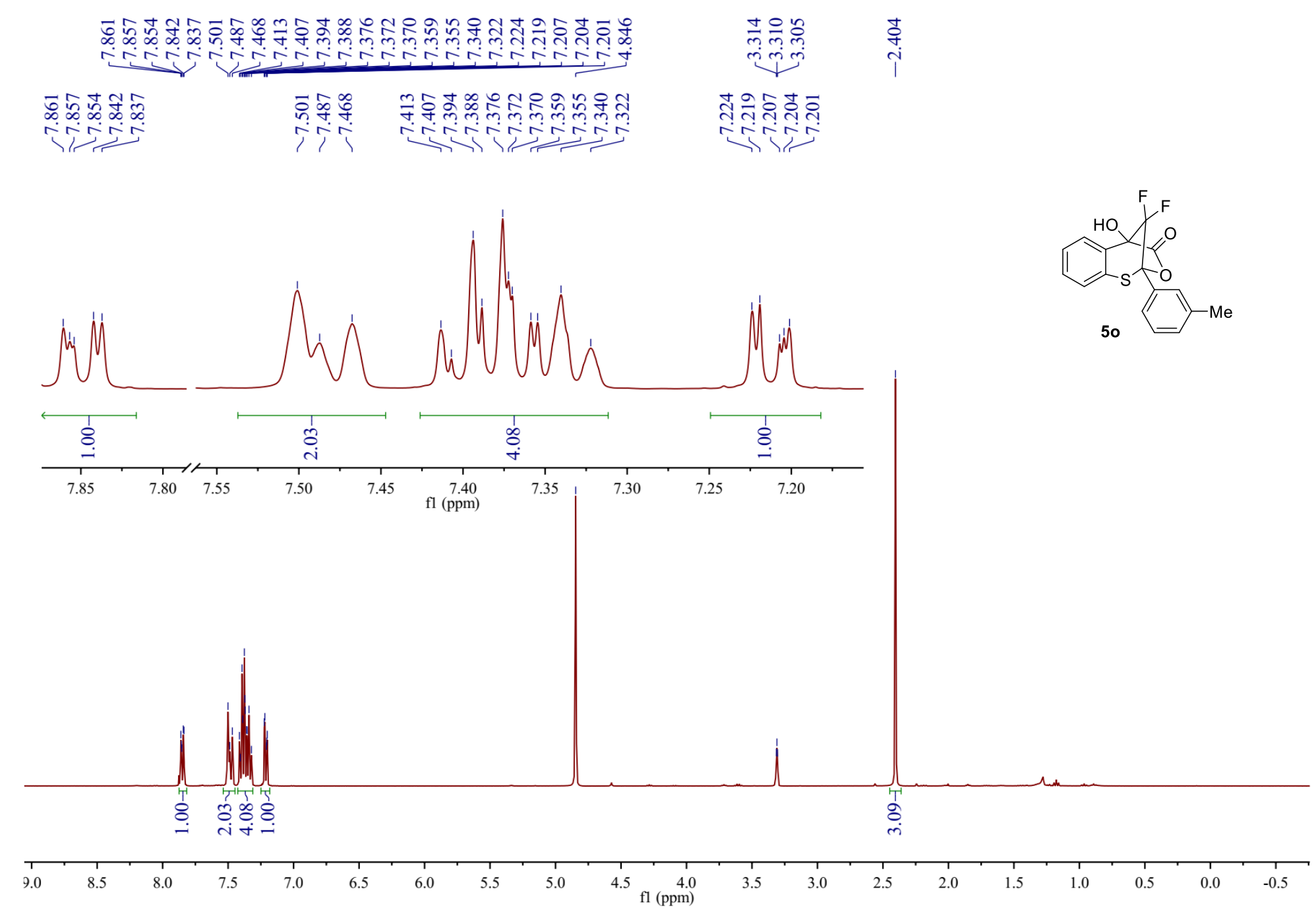

${ }^{1} \mathrm{H}$ NMR spectra of $\mathbf{5 o}$ in $\mathrm{CD}_{3} \mathrm{OD}(400 \mathrm{MHz})$ 

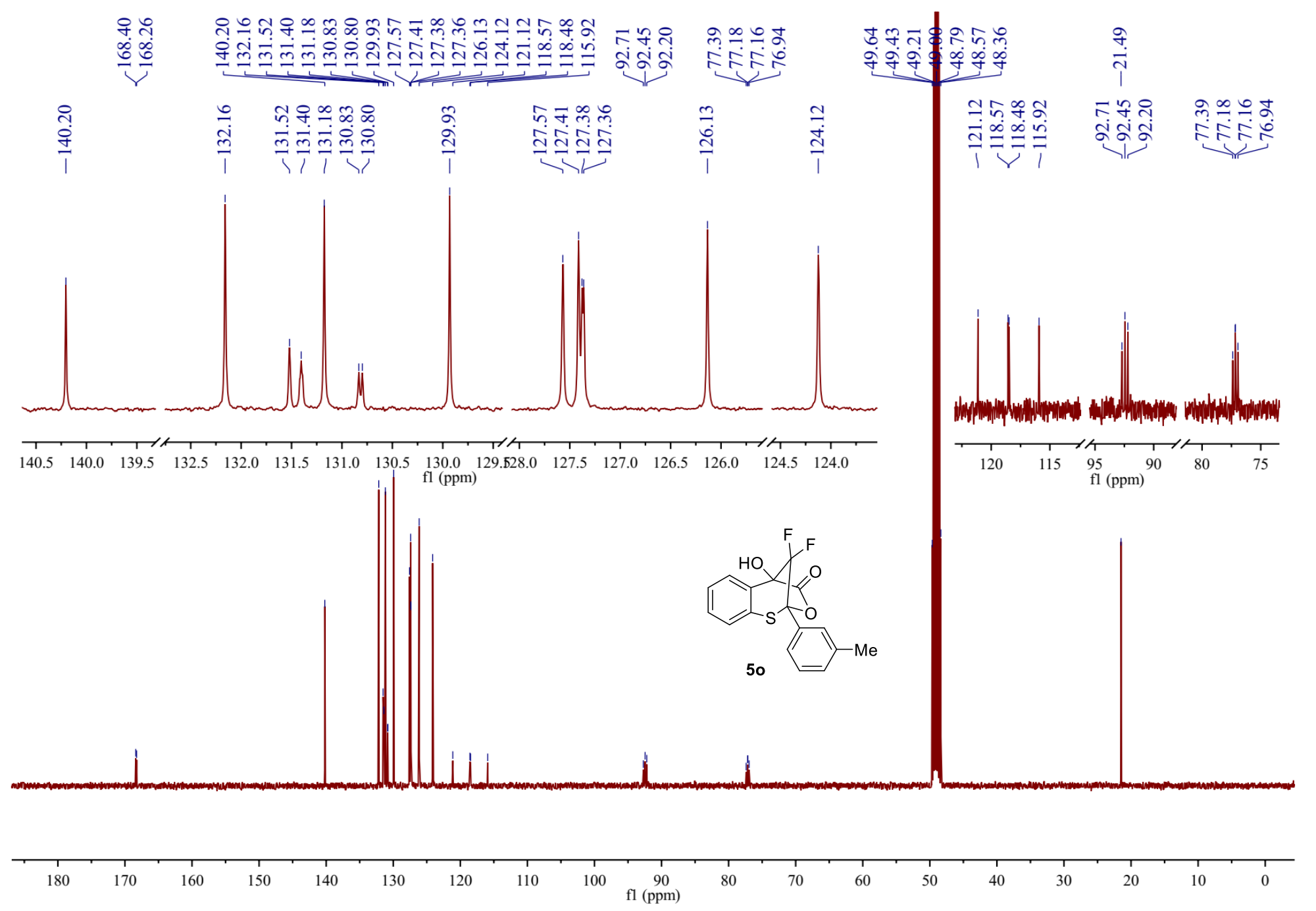

${ }^{13} \mathrm{C}\left\{{ }^{1} \mathrm{H}\right\}$ NMR spectra of 50 in $\mathrm{CD}_{3} \mathrm{OD}(100 \mathrm{MHz})$ 


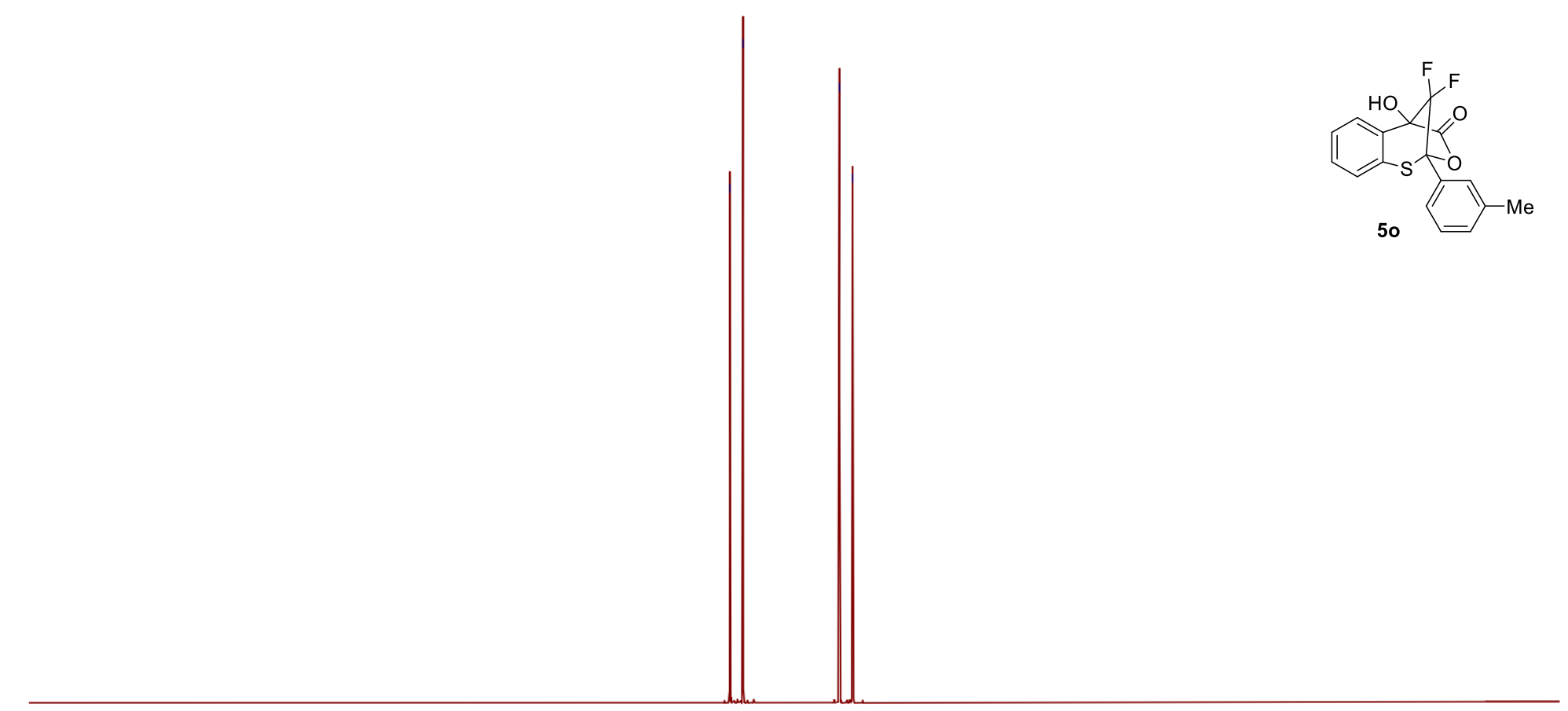

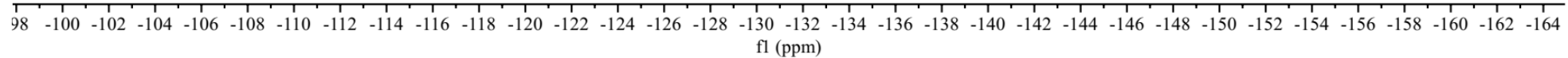

${ }^{19} \mathrm{~F}$ NMR spectra of $5 \mathbf{o}$ in $\mathrm{CD}_{3} \mathrm{OD}(376 \mathrm{MHz})$ 

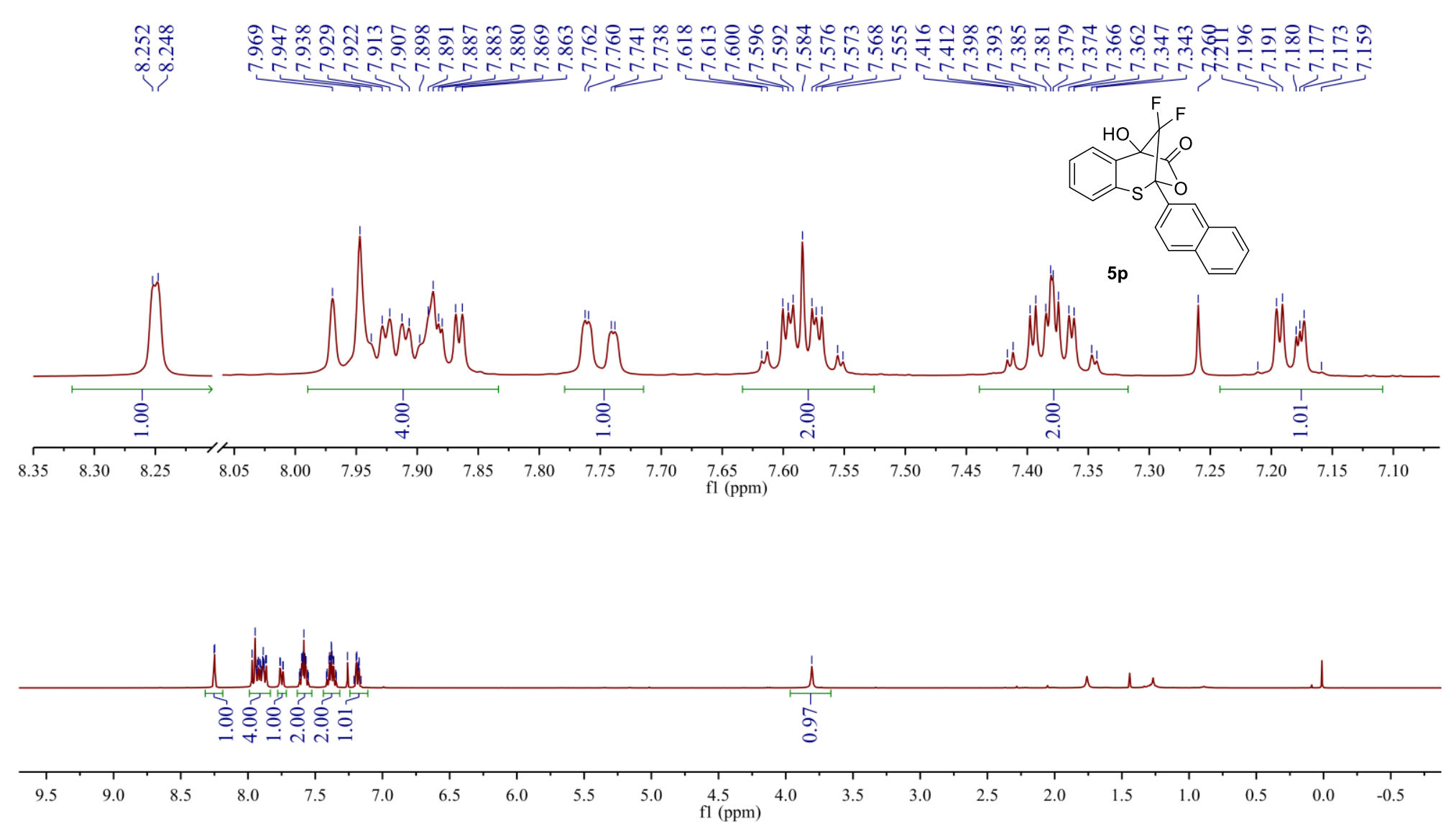

${ }^{1} \mathrm{H}$ NMR spectra of $\mathbf{5 p}$ in $\mathrm{CDCl}_{3}(400 \mathrm{MHz})$ 

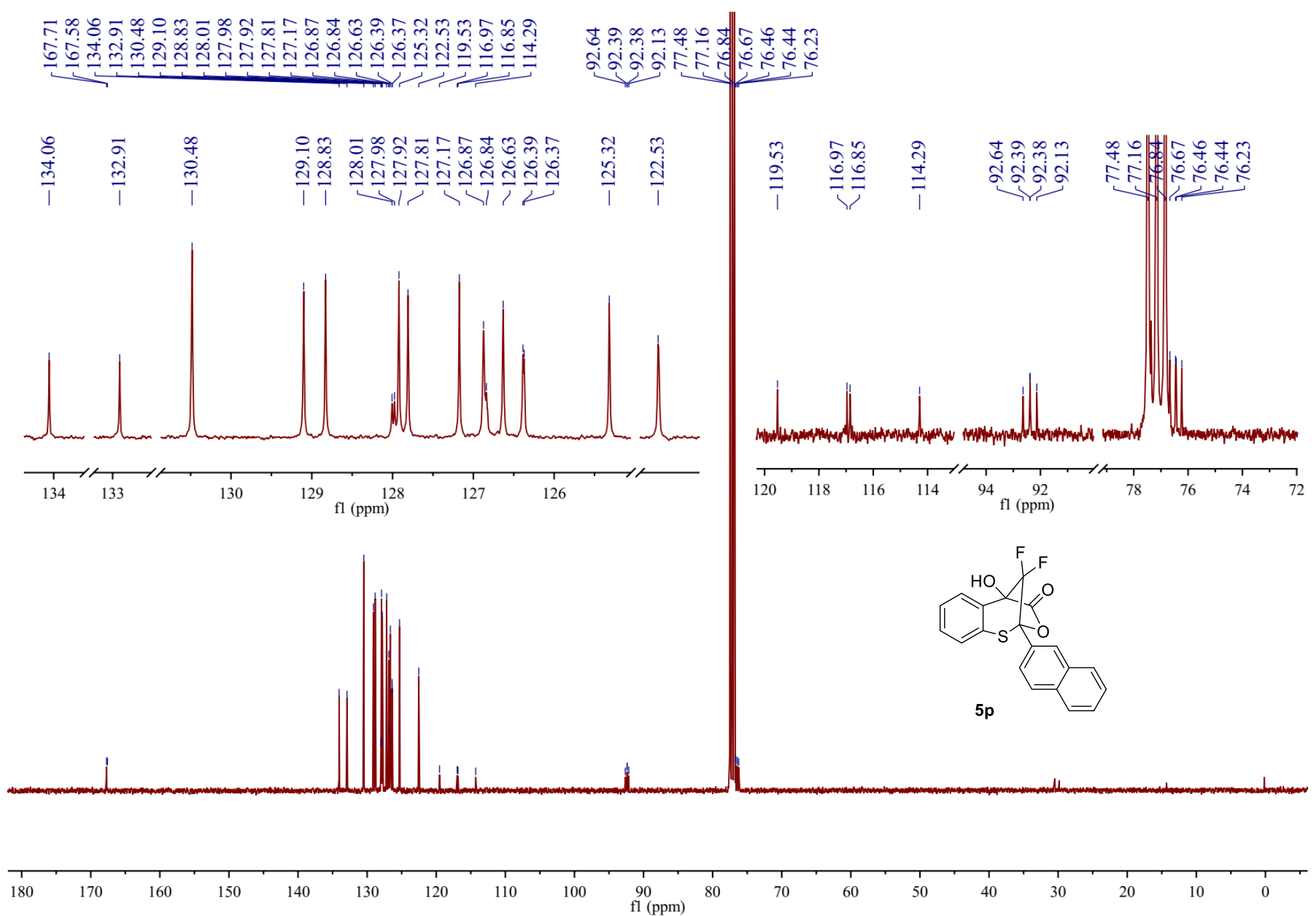

${ }^{13} \mathrm{C}\left\{{ }^{1} \mathrm{H}\right\}$ NMR spectra of $\mathbf{5 p}$ in $\mathrm{CDCl}_{3}(100 \mathrm{MHz})$ 


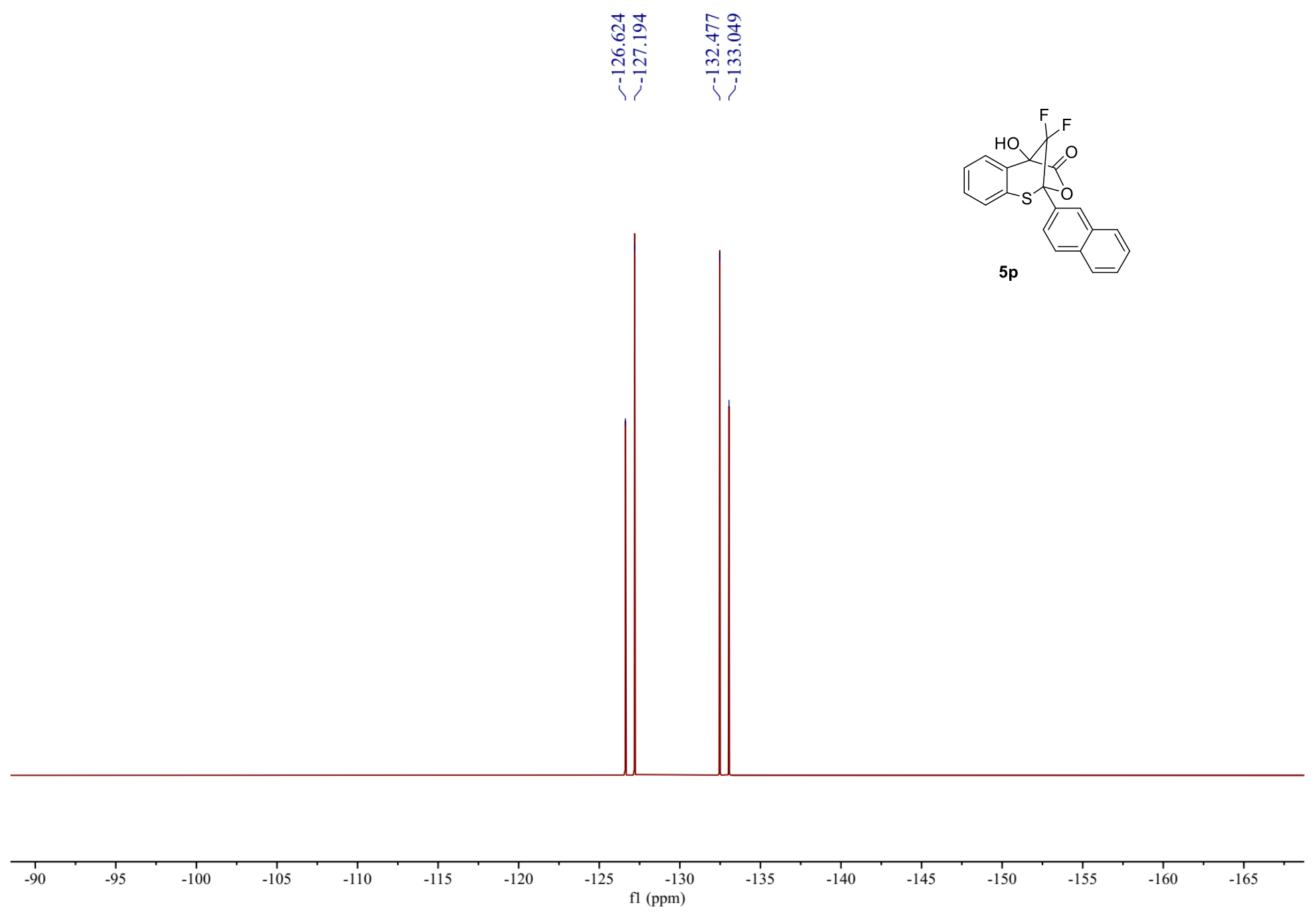

${ }^{19} \mathrm{~F}$ NMR spectra of $\mathbf{5 p}$ in $\mathrm{CDCl}_{3}(376 \mathrm{MHz})$ 


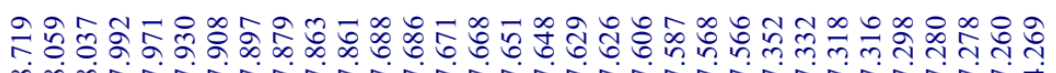

(x)

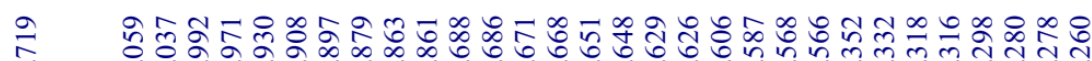

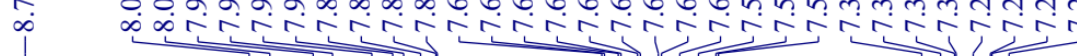
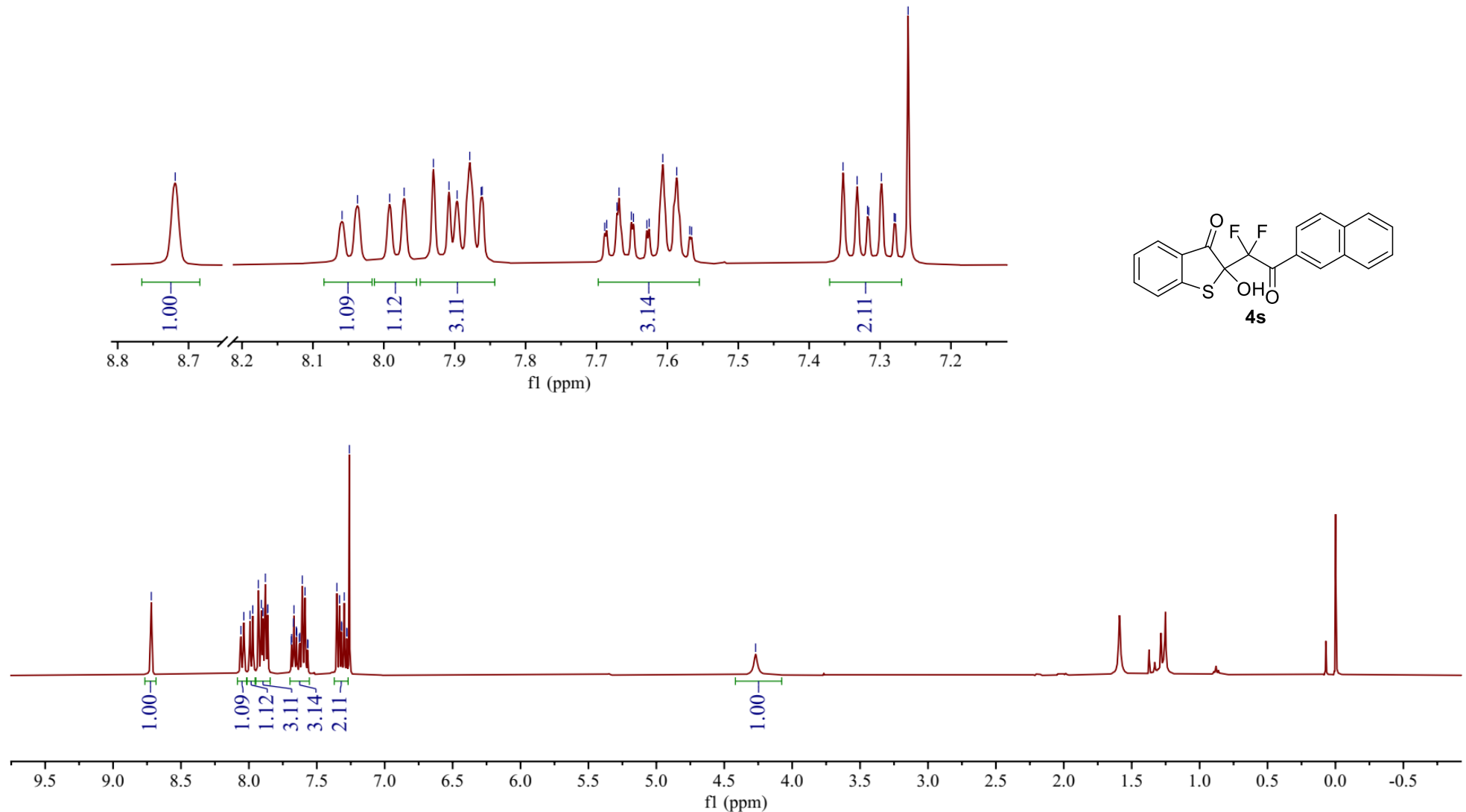

$1.0 \quad 0.5$

${ }^{1} \mathrm{H}$ NMR spectra of $4 \mathbf{s}$ in $\mathrm{CDCl}_{3}(400 \mathrm{MHz})$ 

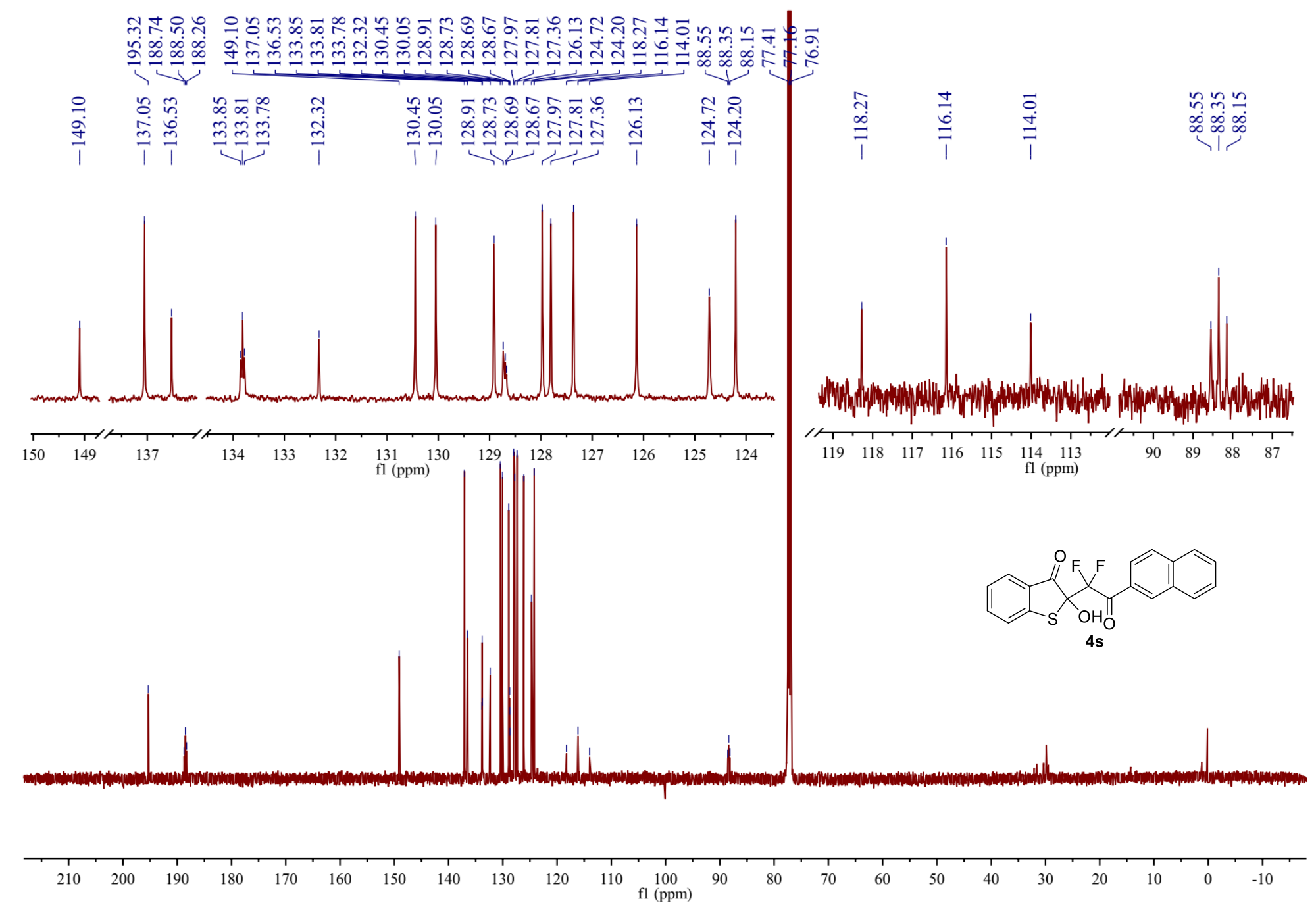

${ }^{13} \mathrm{C}\left\{{ }^{1} \mathrm{H}\right\}$ NMR spectra of $4 \mathrm{~s}$ in $\mathrm{CDCl}_{3}(125 \mathrm{MHz})$ 


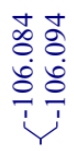
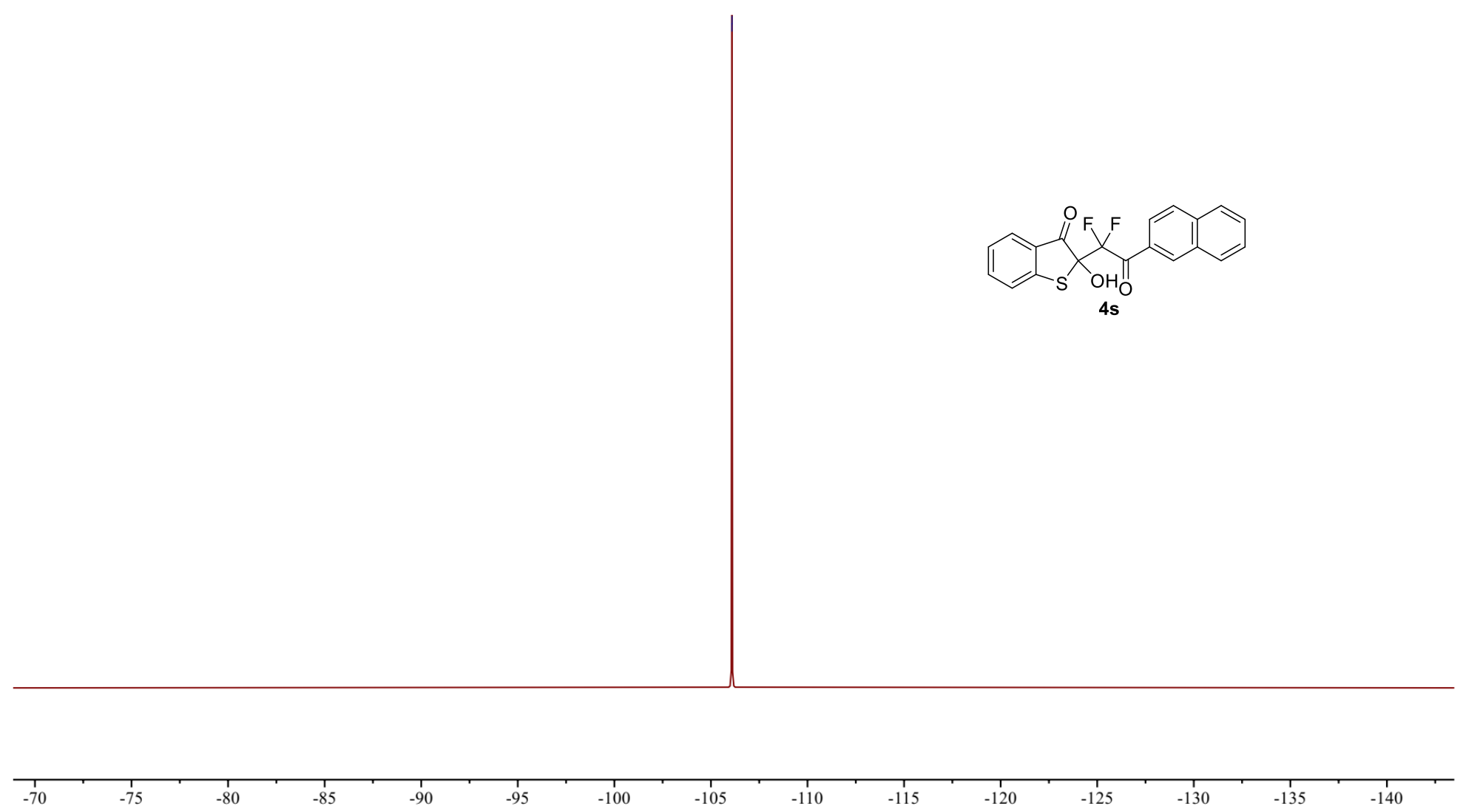

$-90$ $-95$ $-100$ $-105$ $\mathrm{fl}$ (ppm)

${ }^{19} \mathrm{~F}$ NMR spectra of $4 \mathrm{~s}$ in $\mathrm{CDCl}_{3}(376 \mathrm{MHz})$ 


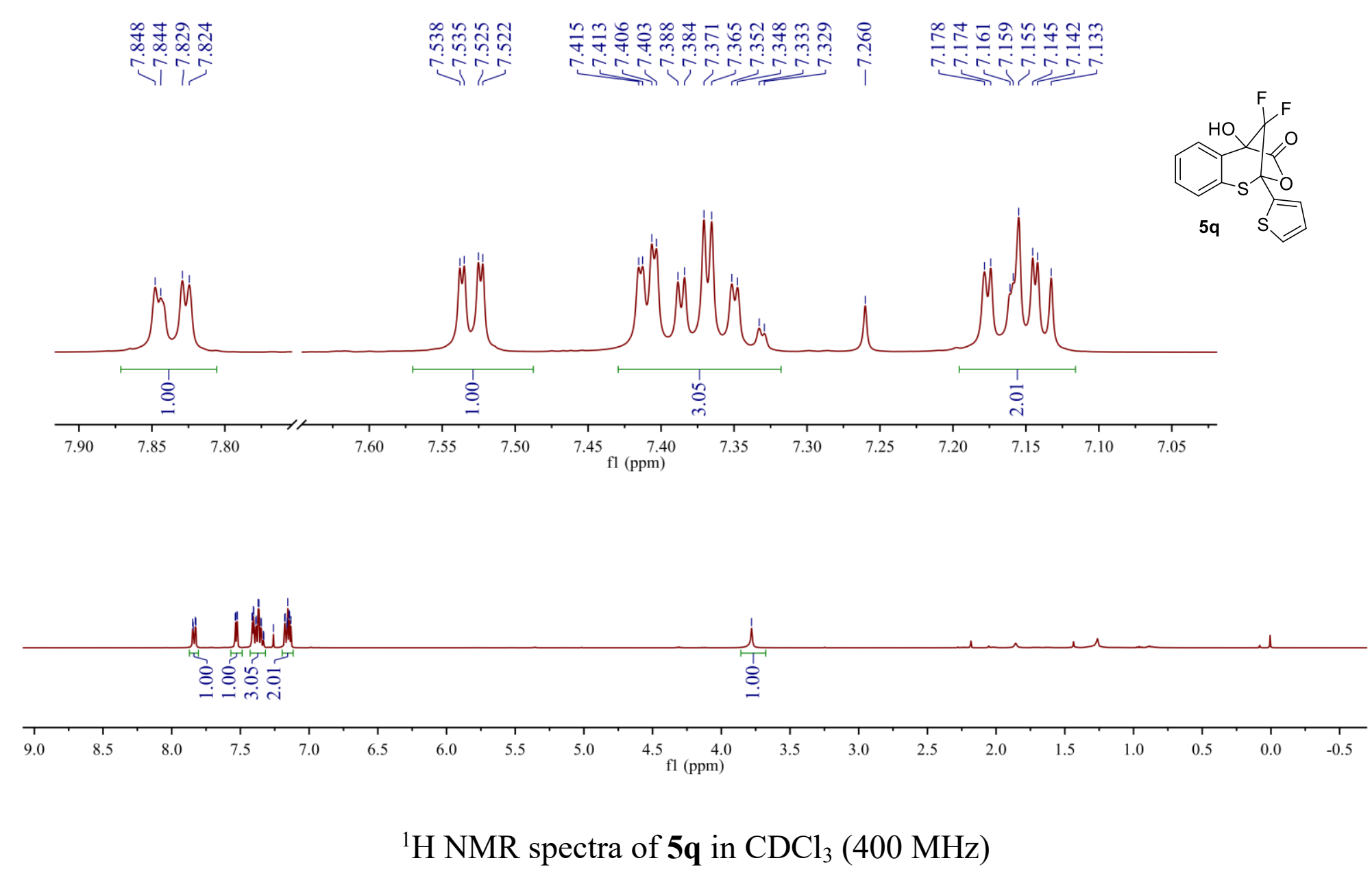



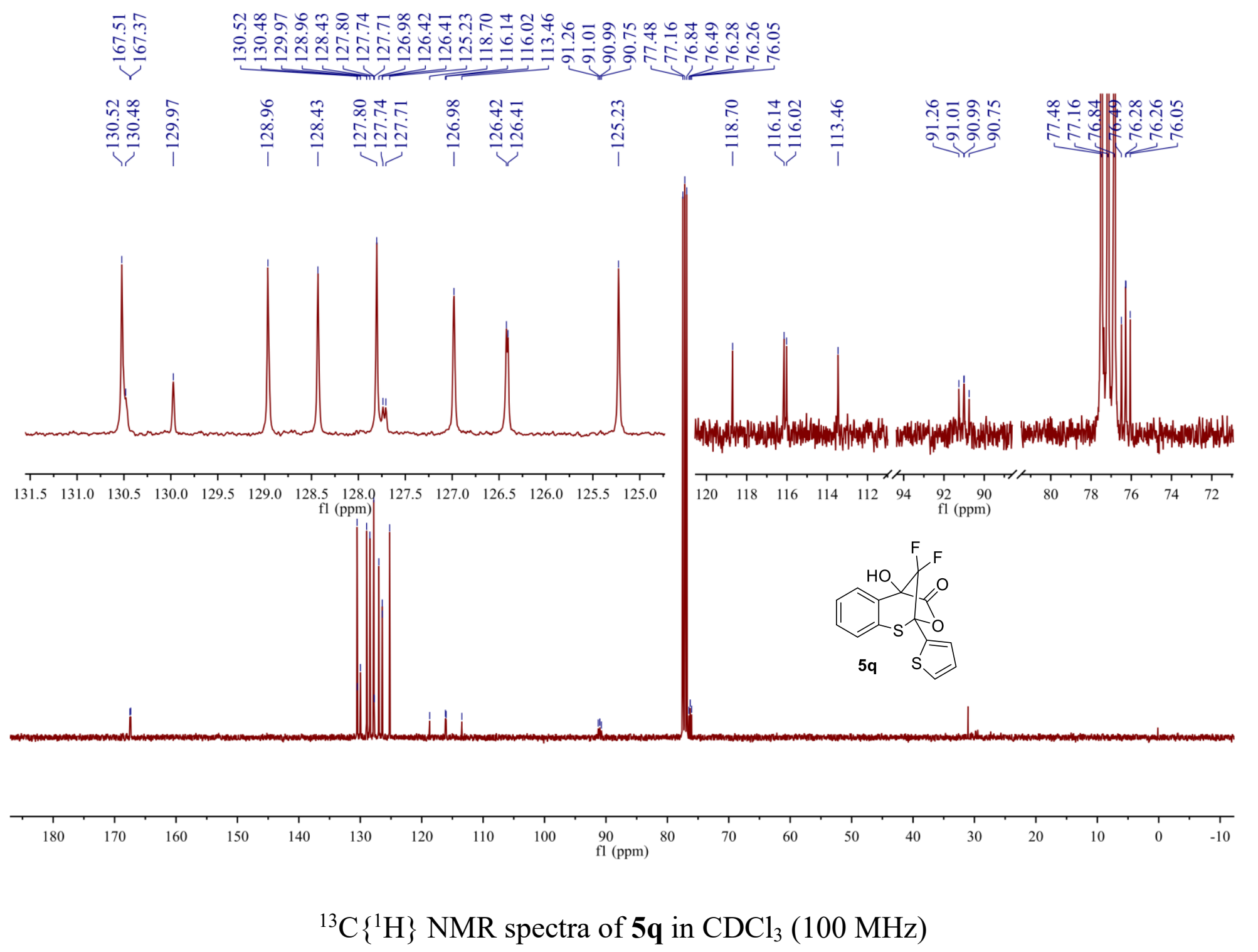


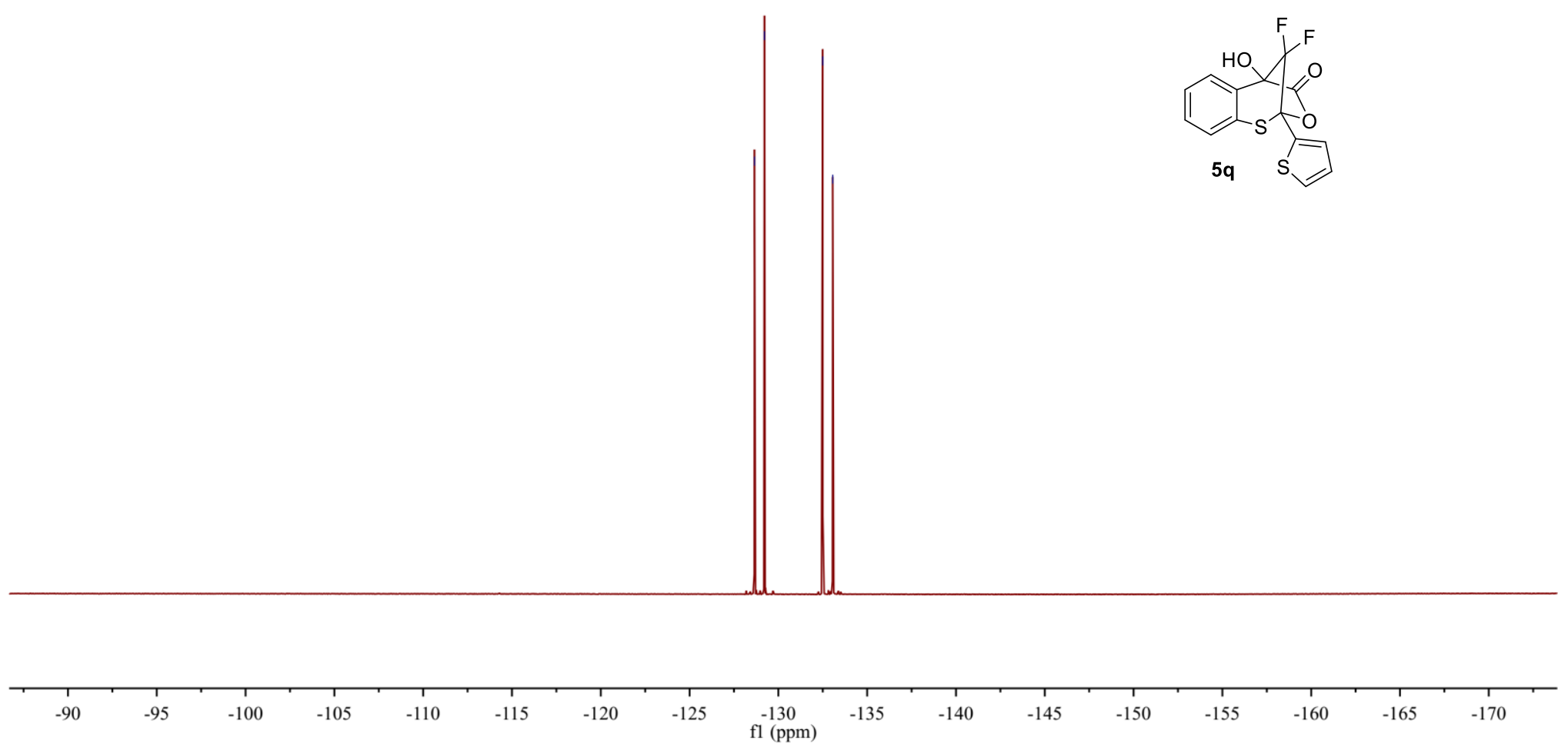

${ }^{19} \mathrm{~F}$ NMR spectra of $\mathbf{5 q}$ in $\mathrm{CDCl}_{3}(376 \mathrm{MHz})$ 
西许
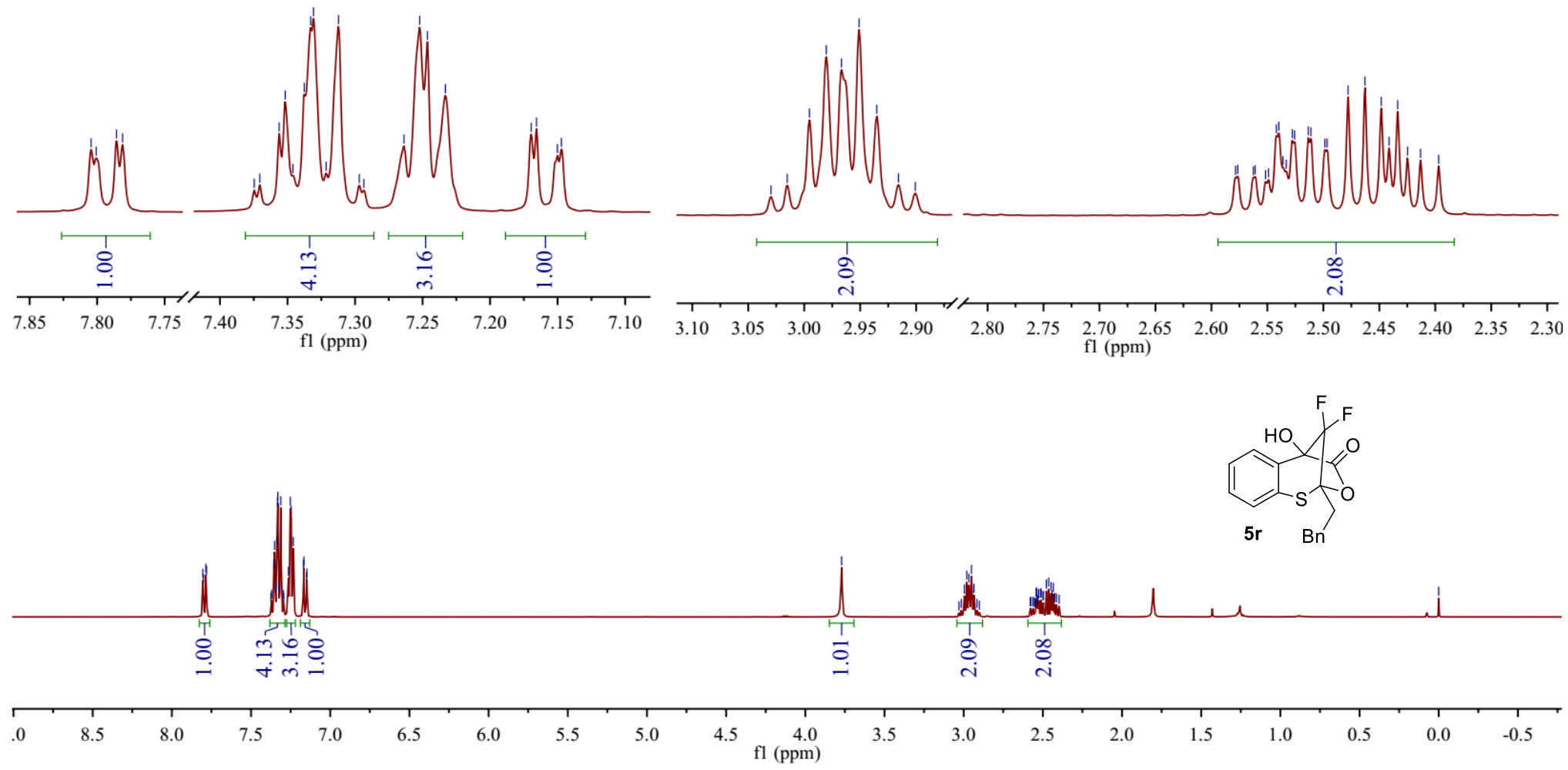

${ }^{1} \mathrm{H}$ NMR spectra of $\mathbf{5}$ in $\mathrm{CDCl}_{3}(400 \mathrm{MHz})$

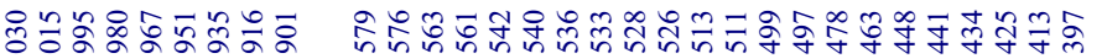

$\mathbf{S 1 9 0}$

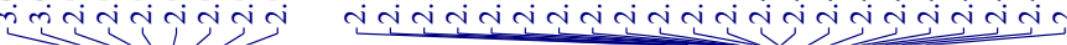



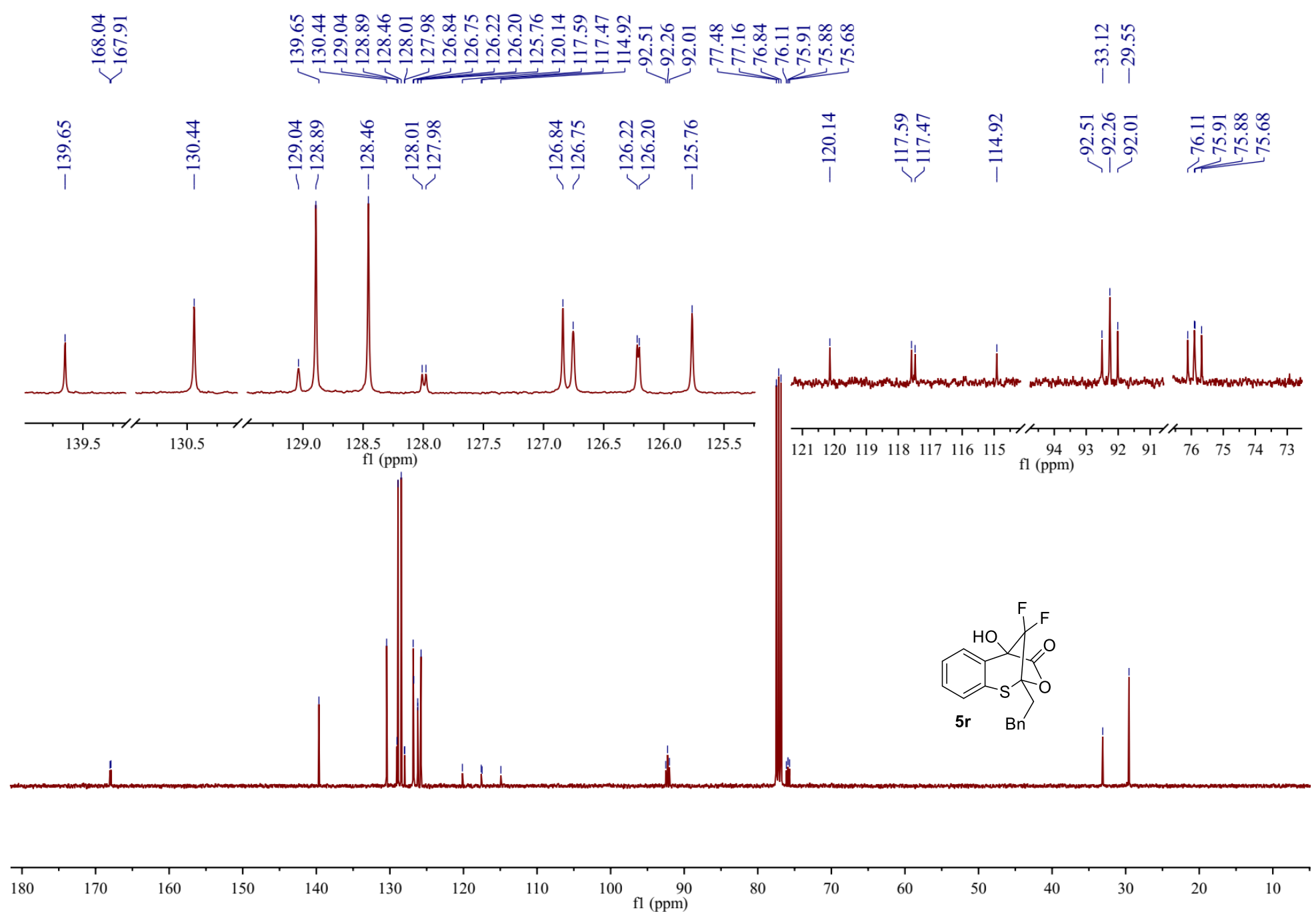

${ }^{13} \mathrm{C}\left\{{ }^{1} \mathrm{H}\right\}$ NMR spectra of $\mathbf{5 r}$ in $\mathrm{CDCl}_{3}(100 \mathrm{MHz})$ 


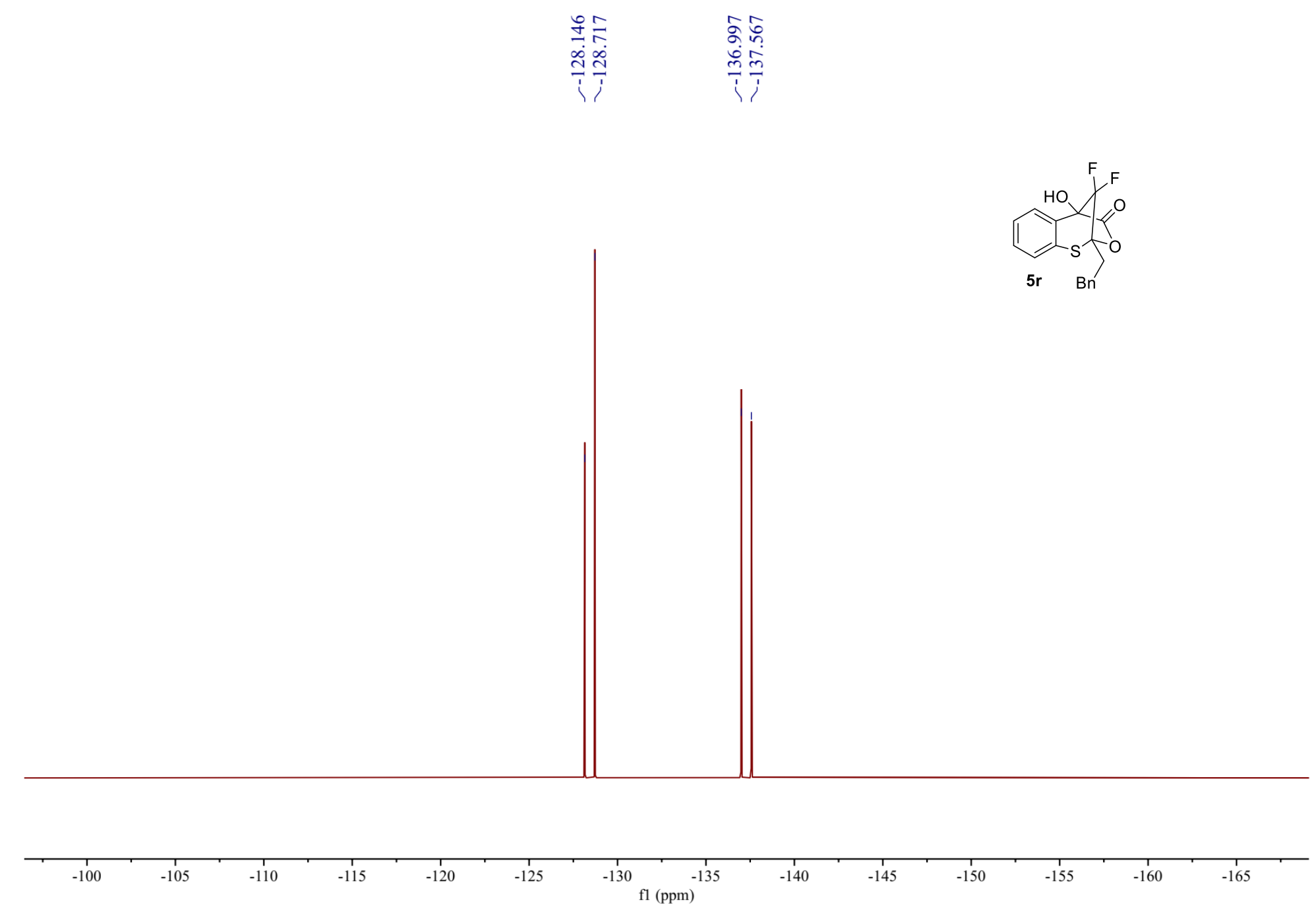

${ }^{19} \mathrm{~F}$ NMR spectra of $\mathbf{5} \mathbf{r}$ in $\mathrm{CDCl}_{3}(376 \mathrm{MHz})$ 


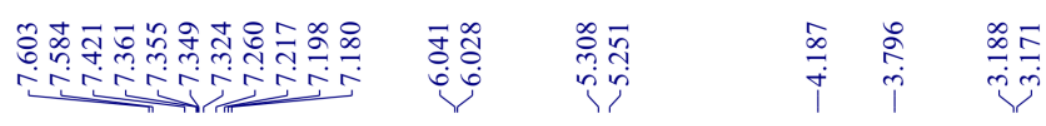
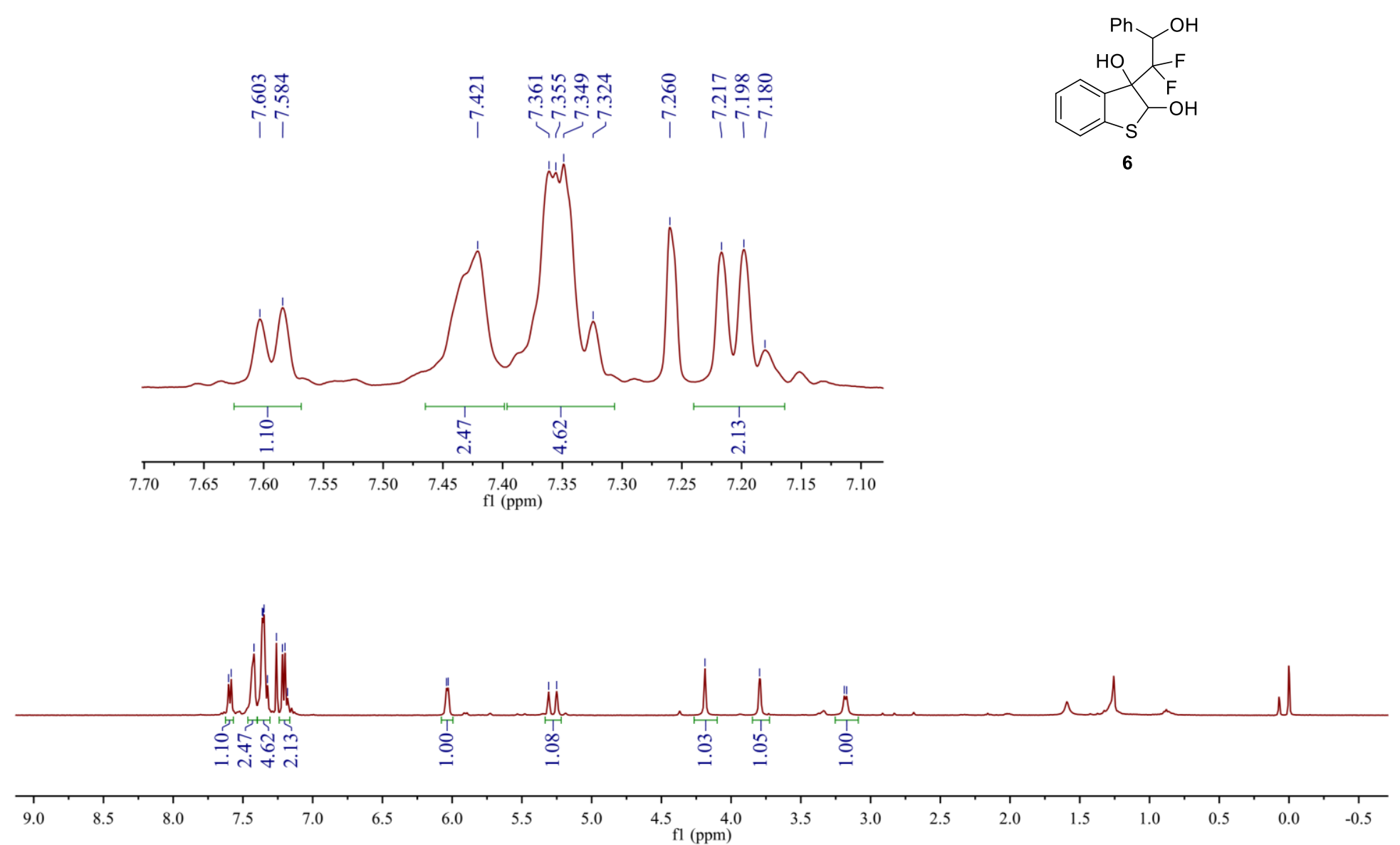

${ }^{1} \mathrm{H}$ NMR spectra of 6 in $\mathrm{CDCl}_{3}(400 \mathrm{MHz})$ 

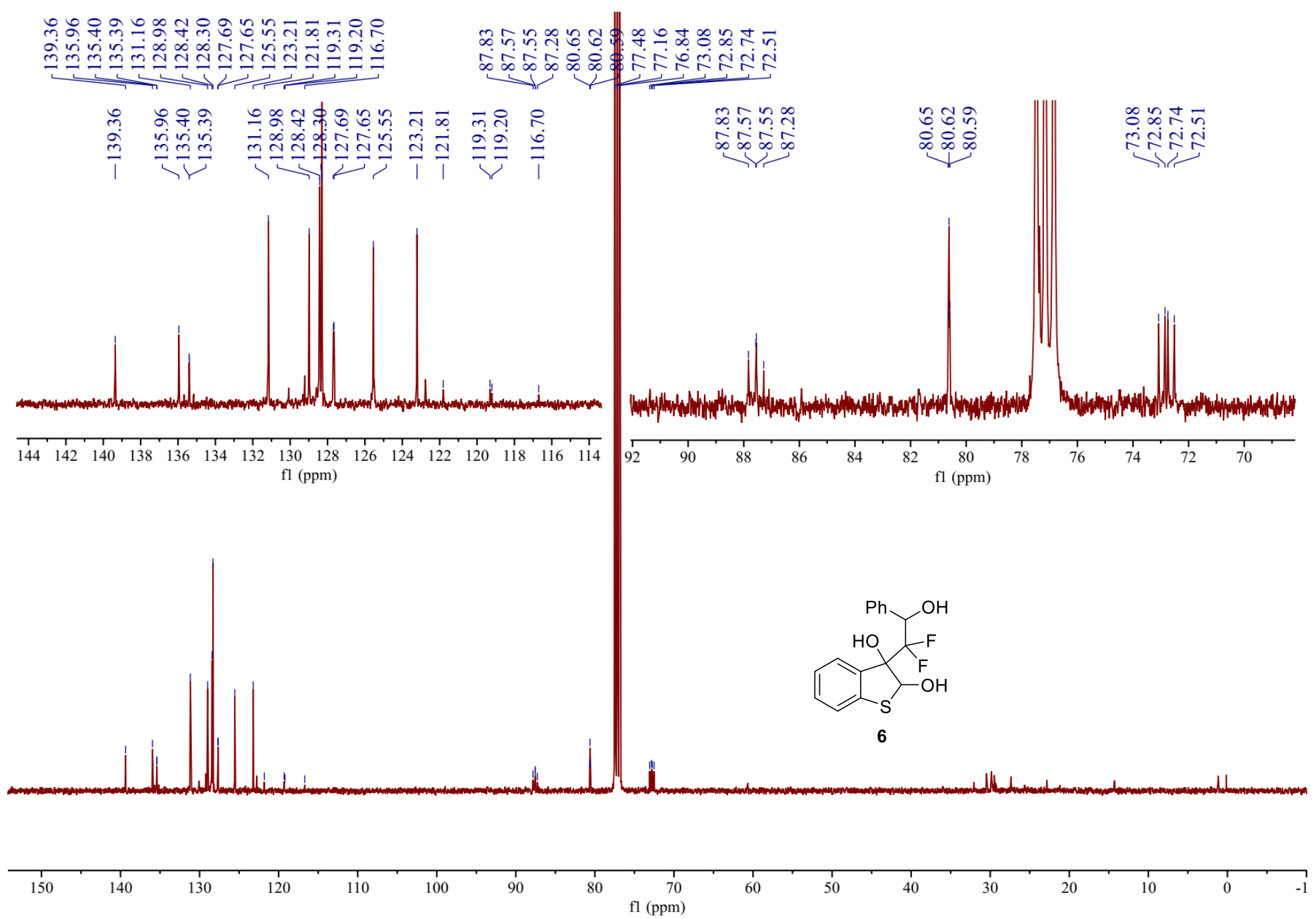

${ }^{13} \mathrm{C}\left\{{ }^{1} \mathrm{H}\right\}$ NMR spectra of $\mathbf{6}$ in $\mathrm{CDCl}_{3}(100 \mathrm{MHz})$ 


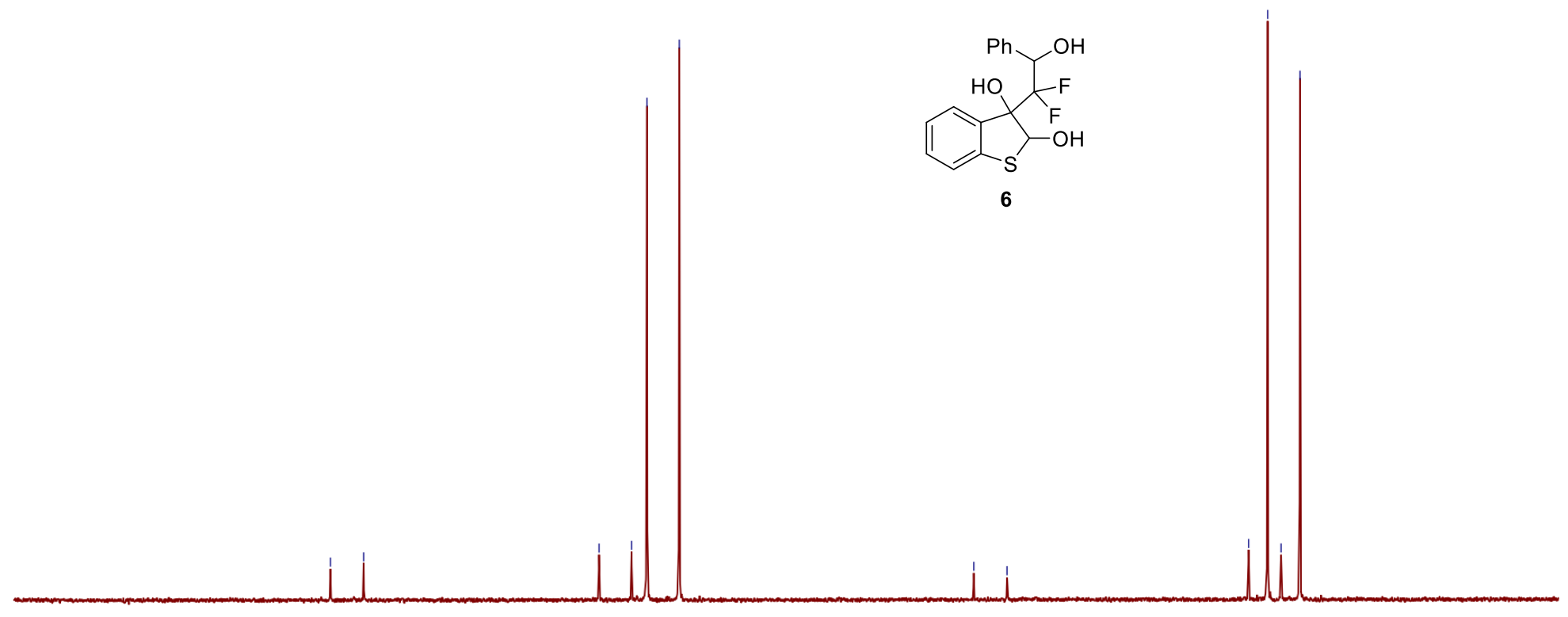

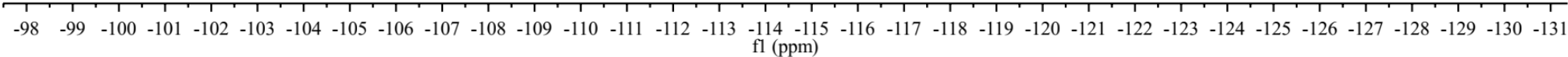

${ }^{19} \mathrm{~F}$ NMR spectra of 6 in $\mathrm{CDCl}_{3}(376 \mathrm{MHz})$ 

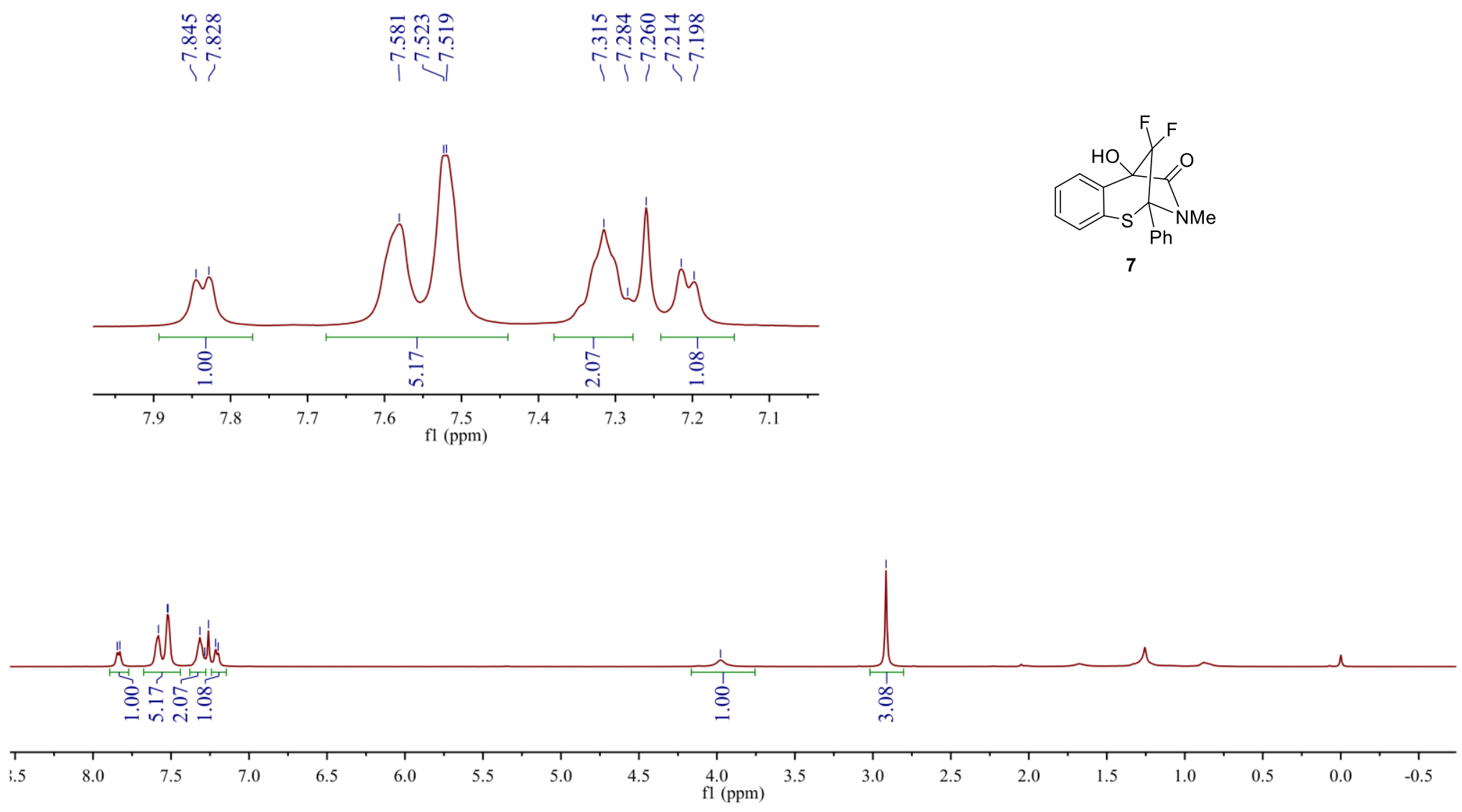

${ }^{1} \mathrm{H}$ NMR spectra of 7 in $\mathrm{CDCl}_{3}(400 \mathrm{MHz})$ 

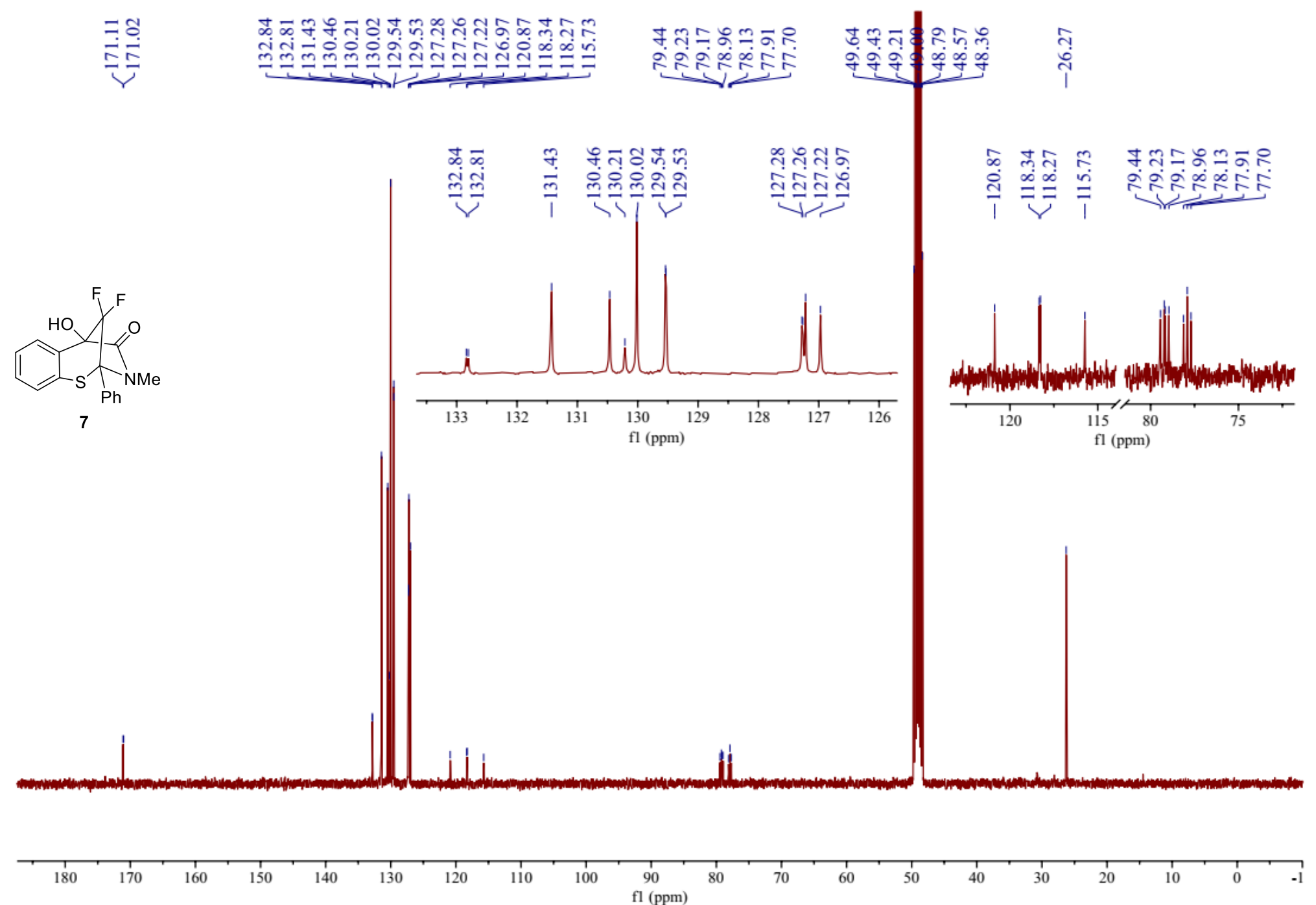

${ }^{13} \mathrm{C}\left\{{ }^{1} \mathrm{H}\right\}$ NMR spectra of 7 in $\mathrm{CD}_{3} \mathrm{OD}(100 \mathrm{MHz})$ 

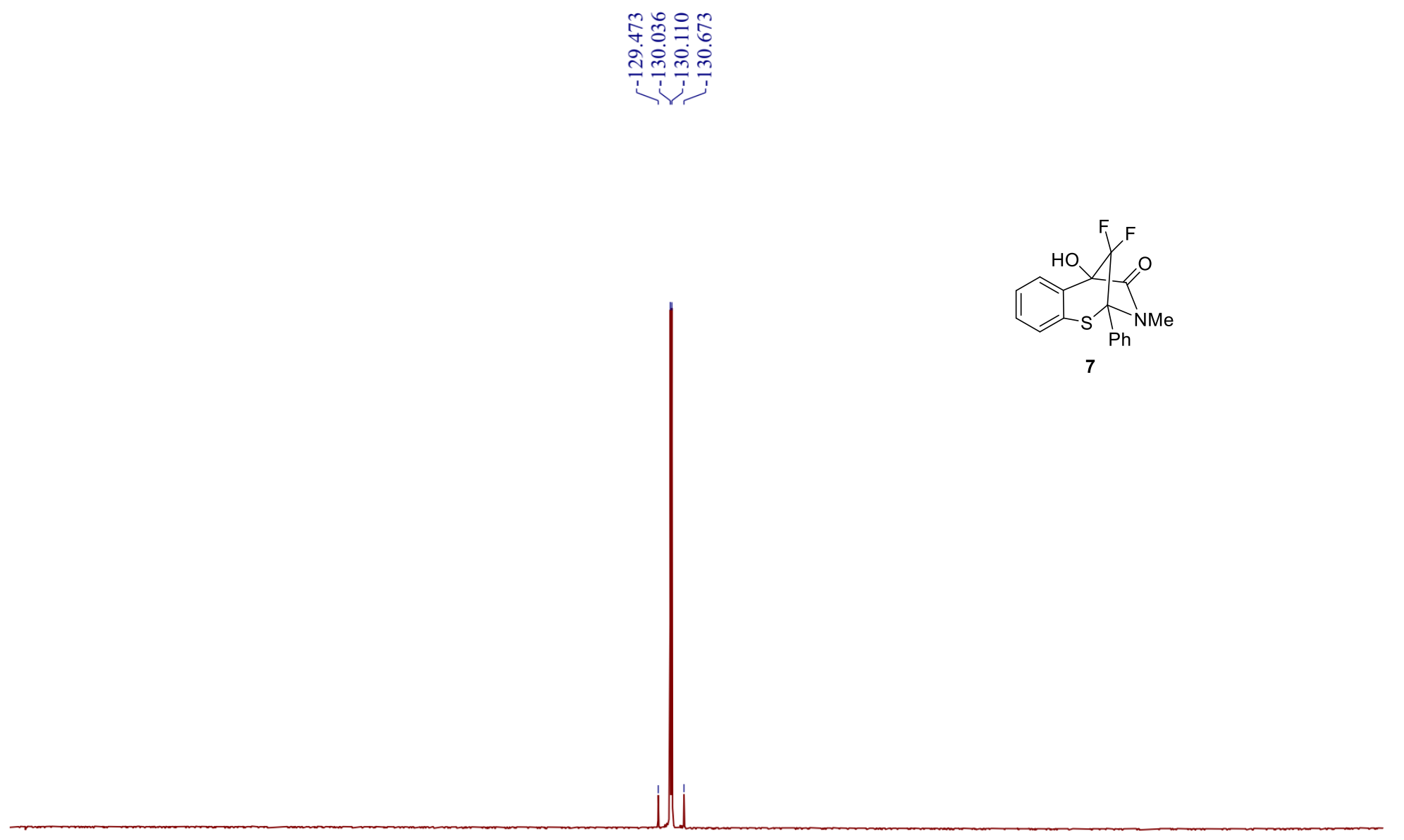

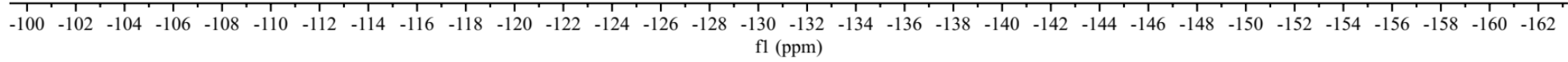

${ }^{19} \mathrm{~F}$ NMR spectra of 7 in $\mathrm{CDCl}_{3}(376 \mathrm{MHz})$ 